Med Klin Intensivmed Notfmed 2020 115 (Suppl 2):S37-S109

https://doi.org/10.1007/s00063-020-00685-0

Online publiziert: 30 . April 2020

(c) Springer Medizin Verlag $\mathrm{GmbH}$, ein Teil von Springer Nature 2020
F. M. Brunkhorst ${ }^{1} \cdot$ M. A. Weigand ${ }^{2} \cdot$ M. Pletz ${ }^{3} \cdot$ P. Gastmeier ${ }^{4} \cdot$ S. W. Lemmen ${ }^{5}$. A. Meier-Hellmann ${ }^{6} \cdot$ M. Ragaller ${ }^{7}$ A. Weyland ${ }^{8} \cdot$ G. Marx ${ }^{9} \cdot$ M. Bucher ${ }^{10}$. H. Gerlach ${ }^{11} \cdot$ B. Salzberger ${ }^{12}$ B. Grabein ${ }^{13} \cdot$ T. Welte $^{14} \cdot$ K. Werdan $^{15} \cdot$ S. Kluge ${ }^{16}$. H. G. Bone ${ }^{17} \cdot$ C. Putensen ${ }^{18} \cdot$ R. Rossaint ${ }^{19} \cdot M$. Quintel $^{20} \cdot$ C. Spies $^{21} \cdot$ B. Weiß ${ }^{21}$. S. John ${ }^{22} \cdot$ M. Oppert ${ }^{23} \cdot$ A. Jörres ${ }^{24} \cdot$ T. Brenner ${ }^{2} \cdot$ G. Elke El $^{25} \cdot$ M. Gründling ${ }^{26}$. K. Mayer ${ }^{27} \cdot$ A. Weimann ${ }^{28} \cdot$ T. W. Felbinger ${ }^{29} \cdot$ H. Axer ${ }^{30} \cdot$ Deutsche Sepsis $^{2}$ Gesellschaft e. V. ${ }^{31}$

'Zentrum für Klinische Studien, Integriertes Forschungs- und Behandlungszentrum (IFB) Sepsis und Sepsisfolgen, Klinik für Anästhesiologie und Intensivmedizin, Universitätsklinikum Jena, Jena, Deutschland; ${ }^{2}$ Klinik für Anästhesiologie, Universitätsklinikum Heidelberg, Heidelberg, Deutschland; ${ }^{3}$ Institut für Infektionsmedizin und Krankenhaushygiene, Universitätsklinikum Jena, Jena, Deutschland; ${ }^{4}$ Institut für Hygiene und Umweltmedizin, Charité - Universitätsmedizin Berlin, Berlin, Deutschland; ${ }^{5}$ Zentralbereich für Krankenhaushygiene und Infektiologie, Universitätsklinikum Aachen, Aachen, Deutschland; ${ }^{6}$ Klinik für Anästhesie, Intensivmedizin und Schmerztherapie, Helios Klinikum Erfurt GmbH, Erfurt, Deutschland; ' ${ }^{7}$ Klinik und Poliklinik für Anästhesiologie und Intensivtherapie, Universitätsklinikum Dresden, Dresden, Deutschland; ${ }^{8} \mathrm{Klinik}$ für Anästhesiologie, Intensivmedizin, Notfallmedizin, Schmerztherapie, Klinikum Oldenburg gGmbH, Oldenburg, Deutschland; ${ }^{9}$ Klinik für Operative Intensivmedizin und Intermediate Care, Universitätsklinikum Aachen, Aachen, Deutschland; ${ }^{10} \mathrm{Klinik}$ für Anästhesiologie und Operative Intensivmedizin, Universitätsklinikum Halle, Halle, Deutschland; " Klinik für Anästhesie, operative Intensivmedizin und Schmerztherapie, Vivantes Klinikum Neukölln, Berlin, Deutschland; ${ }^{12}$ Abteilung für Krankenhaushygiene und Infektiologie, Universitätsklinikum Regensburg, Regensburg, Deutschland; ${ }^{13}$ Stabsstelle Klinische Mikrobiologie und Krankenhaushygiene, Klinikum der Universität München, München, Deutschland; ${ }^{14}$ Klinik für Pneumologie, Medizinische Hochschule Hannover, Hannover, Deutschland; ${ }^{15}$ Universitätsklinik und Poliklinik für Innere Medizin III., Klinikum der MLU Halle-Wittenberg, Halle, Deutschland; ${ }^{16}$ Klinik für Intensivmedizin, Universitätsklinikum HamburgEppendorf, Hamburg, Deutschland; ${ }^{17}$ Zentrum für Anästhesiologie, Intensivmedizin und

Schmerztherapie, Knappschaftskrankenhaus Recklinghausen, Recklinghausen, Deutschland; ${ }^{18} \mathrm{Klinik}$ für Anästhesiologie und Operative Intensivmedizin, Universitätsklinikum Bonn, Bonn, Deutschland; ${ }^{19} \mathrm{Klinik}$ für Anästhesiologie, Universitätsklinikum Aachen, Aachen, Deutschland; ${ }^{20}$ Klinik für Anästhesiologie, Universitätsmedizin Göttingen, Göttingen, Deutschland; ${ }^{21}$ Klinik für Anästhesiologie mit Schwerpunkt operative Intensivmedizin, Charité - Universitätsmedizin Berlin, Berlin, Deutschland; ${ }^{22}$ Klinik für Innere Medizin 8, Schwerpunkt Kardiologie, Klinikum Nürnberg, Nürnberg, Deutschland; ${ }^{23}$ Klinik für Notfall- und Internistische Intensivmedizin, Klinikum Ernst von Bergmann Potsdam, Potsdam, Deutschland; ${ }^{24}$ Klinik für Nephrologie, Transplantationsmedizin und internistische Intensivmedizin, Medizinische Klinik I Krankenhaus Merheim, Klinikum der Universität Witten/Herdecke, Köln, Deutschland; ${ }^{25}$ Klinik für Anästhesiologie und Operative Intensivmedizin, Universitätsklinikum Kiel, Kiel, Deutschland; ${ }^{26}$ Klinik für Anästhesiologie - Anästhesie, Intensiv-, Notfall- und Schmerzmedizin, Universitätsklinikum Greifswald, Greifswald, Deutschland; ${ }^{27}$ Medizinische Klinik und Poliklinik II, Klinikum der Justus-Liebig-Universität Gießen, Gießen, Deutschland; ${ }^{28}$ Klinik für Allgemein-, Viszeral- und Onkologische Chirurgie, Klinikum „St. Georg" Leipzig gGmbH, Leipzig, Deutschland; ${ }^{29}$ Klinik für Anästhesiologie, operative Intensivmedizin und Schmerztherapie, Städtisches Klinikum München, München, Deutschland; ${ }^{30}$ Klinik für Neurologie, Universitätsklinikum Jena, Jena, Deutschland; ${ }^{31}$ Universitätsklinikum Jena, Jena, Deutschland

\section{S3-Leitlinie Sepsis - Prävention, Diagnose, Therapie und Nachsorge}

\section{Langfassung}




\section{Zusatzmaterial online}

Die Onlineversion dieses Beitrags (https://doi. org/10.1007/s00063-020-00685-0) enthält den Leitlinienreport und die Erklärungen zum Interessenkonflikt. Beitrag und Zusatzmaterial stehen Ihnen auf www.springermedizin. de zur Verfügung. Bitte geben Sie dort den Beitragstitel in die Suche ein, das Zusatzmaterial finden Sie beim Beitrag unter „Ergänzende Inhalte".

\section{Beteiligte Fachgesellschaften \\ Deutsche Sepsis Gesellschaft e.V. \\ (federführend) \\ Deutsche Gesellschaft für Anästhesiologie \\ und Intensivmedizin e. V. (DGAI) \\ Deutsche Gesellschaft für Internistische \\ - T. Ernährung \\ - U. Setzen von Behandlungszielen \\ - Spätfolgen der Sepsis \\ - Abkürzungen \\ - Anhang \\ - Literatur}

Intensivmedizin und Notfallmedizin (DGIIN)

Deutsche Gesellschaft für Ernährungsmedizin e. V. (DGEM)

Deutsche Gesellschaft für Neurologie (DGN)

Deutsche Gesellschaft für Kardiologie, Herz-

und Kreislaufforschung e. V. (DGK)

Deutsche Gesellschaft für Innere Medizin e.V. (DGIM)

Deutsche Gesellschaft für Infektiologie (DGI) Deutsche Gesellschaft für Nephrologie e.V. (DGfN)

Deutsche Interdisziplinäre Vereinigung für Intensiv- und Notfallmedizin (DIVI)

Deutsche Gesellschaft für Pneumologie und

Beatmungsmedizin (DGP)

Deutsche Gesellschaft für Hygiene und

Mikrobiologie (DGHM)

Deutsche Sepsis Hilfe e. V. (DSH)

Nationales Referenzzentrum für Surveillance von nosokomialen Infektionen (NRZ)

Paul-Ehrlich-Gesellschaft für Chemotherapie e. V. (PEG)

\section{Inhalt}

- 1. Einleitung
- 1. Einleitung
- 2. Definition der Sepsis
- 3 Empfehlungen
- Prävention der Sepsis
- Impfungen
- A. Initiale hämodynamische Stabi-
lisierung
- B. Leitlinienimplementierung
- C. Diagnose
- D. Antimikrobielle Therapie
- E. Fokuskontrolle
- F. Flüssigkeitstherapie
- G. Vasoaktive Medikation
- H. Kortikosteroide
- I. Blutprodukte

- J. Immunglobuline

- K. Blutreinigung

- L. Antikoagulanzien

- M. Invasive Beatmung

- N. Sedierung und Analgesie

- O. Blutzuckerkontrolle

- P. Nierenersatztherapie

- Q. Bikarbonattherapie

- R. Prophylaxe einer venösen Thromboembolie

- S. Stressulkusprophylaxe

\section{Einleitung}

Bei einer Sepsis handelt es sich um eine lebensbedrohliche Organdysfunktion ausgelöst durch eine Infektion, die mit einer Regulationsstörung beim Wirt einhergeht. Ähnlich wie bei Polytrauma, akutem Herzinfarkt oder Schlaganfall können das frühzeitige Erkennen einer Sepsis und deren Behandlung in den ersten Stunden nach ihrem Auftreten die schwerwiegenden Folgen einer Infektion verhindern oder abschwächen.

Die Leitlinie fasst angemessene, wissenschaftlich begründete und aktuelle Verfahren für Diagnostik, Therapie und Nachsorge zusammen und ergänzt diese um Maßnahmen der Sepsisprävention. Die Empfehlungen der Leitlinie sollen als unterstützende Informations- und Entscheidungsgrundlage für den Kliniker zur Behandlung von erwachsenen Patienten mit Sepsis oder septischem Schock dienen. Sie ersetzen nicht die Fähigkeiten des Arztes, eine angemessene Entscheidung bei der individuellen Behandlung eines Patienten nach Maßgabe der verfügbaren klinischen Parameter zu treffen.

Die vorliegende interdisziplinäre Leitlinie der Klassifikation S3 ist ein evidenzund konsensbasiertes Instrument zur Verbesserung und Qualitätssicherung von Prävention, Diagnostik, Therapie und Nachsorge der Sepsis. Sie richtet sich an alle damit betrauten Berufsgruppen sowie an Betroffene, übergeordnete Organisationen (z.B. Krankenkas- sen und Einrichtungen der ärztlichen Selbstverwaltung) und die interessierte Fachöffentlichkeit.

\section{Definition der Sepsis}

Die dieser Leitlinie zugrunde liegende Definition der Sepsis basiert auf den von der Sepsis-3-Taskforce und der Surviving Sepsis Campaign (SSC) veröffentlichten Definitionen $[1,2]$. Sie wird in der vorliegenden Leitlinie nicht als Empfehlung, sondern als Statement (Stellungnahme) beschrieben. Zudem wird mithilfe eines Algorithmus (Sequential Organ Failure Assessment, SOFA-Score) die Möglichkeit der Diagnostik einer sepsisassoziierten Organdysfunktion aufgezeigt. Der Begründungstext wurde von der DSGLeitlinienkommission neu hinzugefügt.

Studien, die als Evidenz für die SSCLeitlinie und die vorliegende Leitlinie herangezogen wurden, beziehen sich $\mathrm{zu}$ einem großen Teil auf Patientenpopulationen, die anhand überholter Definitionen der Sepsis, schweren Sepsis und des septischen Schocks identifiziert wurden [3].

\section{Definition 1 | Statement | 2018}

\section{- Expertenkonsens}

- Eine Sepsis ist eine akut lebensbedrohliche Organdysfunktion, hervorgerufen durch eine inadäquate Wirtsantwort auf eine Infektion. Für die Diagnose einer sepsisassoziierten Organdysfunktion ist eine Veränderung des Sequential Organ Failure Assessment (SOFA) Score um $\geq 2$ Punkte zu verwenden. (- Abb. 1, Anhang)

- DSG-Leitlinienstellungnahme

- Konsensstärke: $100 \%$

Begründung. Die überholten Sepsisdefinitionen (Sepsis-1 von 1992 und Sepsis-2 von 2001) beruhten auf dem SIRS-Konzept (systemisches inflammatorisches Responsesyndrom; $[1,4,5])$. Die SIRSKriterien, die eine Hypo- $\left(<36^{\circ} \mathrm{C}\right)$ oder Hyperthermie $\left(>38^{\circ} \mathrm{C}\right)$, Tachykardie ( $>90 / \mathrm{min})$, Tachypnoe $(>20 / \mathrm{min})$ sowie eine Leukozytose $>12.000 / \mu$ l oder Leukopenie $<4000 / \mu \mathrm{l}$ und/oder Linksver- 
schiebung $>10 \%$ umfassen, sind weder spezifisch noch besonders sensitiv für Infektionen. So berichteten Churpek et al., dass $50 \%$ der Krankenhauspatienten SIRS mindestens einmal während ihres Krankenhausaufenthalts aufwiesen, auch wenn viele dieser Patienten keine Infektion hatten und keine antiinfektive Therapie benötigten [6]. Kaukonen et al. haben gezeigt, dass einer von 8 Intensivpatienten mit Infektionsverdacht und neu aufgetretenem Organversagen weniger als 2 SIRS-Kriterien erfüllt [7]. Diese Patientengruppe wurde gleichwohl als „septisch“ eingestuft und entsprechend behandelt. Epidemiologische Analysen von Krankenhausdaten, die bei der Erfüllung von nur 2 SIRS-Kriterien bereits eine Codierung als Sepsis gestatteten, haben somit zu einer "Verwässerung“ der realen Häufigkeit von und der Sterblichkeit durch Sepsis geführt. So konnten Gaieski et al. für die USA zeigen, dass die relative Krankenhaussterblichkeit in den Jahren 2004-2009 zwar zurückging, die absolute Sterblichkeit aber kontinuierlich stieg [8].

Als Reaktion auf diese Inkonsistenzen erarbeitete die Sepsis-3-Taskforce, eine internationale Arbeitsgruppe der European Society of Intensive Care Medicine (ESICM) und der Society of Critical Care Medicine (SCCM), im Februar 2016 die Neudefinition der Sepsis [1, 4, 6, 9-11]. Erstmals wurde die Sepsis im gesamten Krankenhaus, also auch auf Normalstationen und in Notaufnahmen, berücksichtigt. Sepsis wird demnach immer durch eine akut lebensbedrohliche weil dysregulierte Wirtsreaktion (Organdysfunktion) auf eine Infektion verursacht. Der Begriff der „schweren“ Sepsis entfällt, weil es eine „leichte“ Sepsis in dem neuen Konzept nicht gibt. Die Autoren schlugen vor, stattdessen folgerichtig von einer „Infektion“ zu sprechen. Basierend auf routinemäßig erhobenen klinischen Daten identifizierten die Autoren robuste Risikofaktoren, die über das Risiko zur Baseline hinaus den Übergang von einer lokal begrenzten Infektion mit geringem Sterberisiko zu einer Sepsis mit hohem Sterberisiko anzeigen. Hierzu waren geeignete statistische Verfahren erforderlich, da es gegenwärtig keinen Goldstandard für die Diagnose einer
Sepsis gibt. Nur wenn ein Goldstandard existiert, ist die Berechnung von Sensitivität und Spezifität auf Grundlage folgender Zuordnungen möglich: richtig-positive (Fälle mit Sepsis), richtig-negative (Kontrollen ohne Sepsis) bzw. falsch-positive und falsch-negative. Stattdessen musste die Aussagekraft eines Tests unter verschiedenen Aspekten der Validität, Reliabilität und Nützlichkeit beurteilt werden. Aus den ermittelten Risikofaktoren wurde der Quick-Sequential-Organ-Failure-Assessment(qSOFA)Score abgeleitet, dessen Stärke vor allem in der prädiktiven Validität liegt. Wichtigster Outcomeparameter ist die Krankenhaussterblichkeit, weil die Sepsis im Gegensatz zu einer lokal begrenzten Infektion ohne Organdysfunktion immer lebensbedrohlich ist. Da allerdings die Sterblichkeit nicht immer durch eine Sepsis verursacht sein muss, wurden neben der ,area under the receiver operating characteristic curve" (AUROC) auch die Änderungen innerhalb jedes Dezils, am Baselinerisiko zu versterben, erfasst.

Für die Feststellung der sepsisassoziierten Organdysfunktion wird eine Veränderung des SOFA-Scores um $\geq 2$ Punkte vorgeschlagen. Ein SOFAScore von $\geq 2$ entspricht einem Letalitätsrisiko von über $10 \%$ bei stationären Patienten außerhalb der Intensivstation (ITS), die eine Infektion haben. Der SOFA-Score, der 6 Organsysteme nach 4 Schweregraden der Organdysfunktion einstuft und 0-24 Punkte umfasst, ist jedoch aufwändig zu ermitteln und daher in der klinischen Routine außerhalb der ITS zum bettseitigen Screening ungeeignet. Daher hat die Sepsis-3Arbeitsgruppe den qSOFA entwickelt, um Patienten mit hohem Sepsisrisiko schneller zu identifizieren.

\section{Definition 2 | Statement | 2018}

- Expertenkonsens

- Ein septischer Schock ist definiert als eine trotz adäquater Volumentherapie persistierende arterielle Hypotension mit der Notwendigkeit einer Therapie mit Vasopressoren, um einen mittleren arteriellen Blutdruck von $\geq 65 \mathrm{~mm} \mathrm{Hg}$ zu erreichen.
Gleichzeitig muss der Laktatwert im Serum $>2 \mathrm{mmol} / \mathrm{l}$ betragen. (- Abb. 1, Anhang)

- DSG-Leitlinienstellungnahme

- Konsensstärke: $100 \%$

Begründung. Ein systematisches Review, das die Operationalisierung von gegenwärtigen Definitionen des septischen Schocks untersuchte, zeigte eine erhebliche Heterogenität der berichteten Sterblichkeitsraten auf. Diese Heterogenität resultierte $u$. a. aus Unterschieden in den ausgewählten klinischen Variablen (unterschiedliche Cut-off-Werte für den systolischen oder mittleren Blutdruck, unterschiedliche Level von Hyperlaktatämie und Vasopressordosis). Unter Verwendung der o.g. Definition (mittlerer arterieller Blutdruck von $\geq 65 \mathrm{~mm} \mathrm{Hg}$ und Laktatwert im Serum $>2 \mathrm{mmol} / \mathrm{l}$ ) wurden in großen klinischen Kohorten, Notaufnahmen und Normalstationen Krankenhaussterblichkeitsraten von mehr als $40 \%$ festgestellt.

\section{Empfehlungen}

\section{Prävention der Sepsis}

Die Leitlinienkommission hat einvernehmlich entschieden, der Prävention nosokomialer Infektionen wegen ihrer großen Bedeutung ein eigenes Kapitel zu widmen. In den Empfehlungen der SSC werden Maßnahmen zur Prävention von nosokomialen Infektionen nicht separat aufgegriffen.

Zur Prävention von nosokomialen Infektionen, die zu einer Sepsis führen können, gibt es diverse spezifische Leitlinien und Empfehlungen. Für Deutschland sind hier insbesondere die Empfehlungen der Kommission für Krankenhaushygiene und Infektionsprävention (KRINKO) zu erwähnen. Es wurde beschlossen, an dieser Stelle nur zu den wichtigsten Kernkomponenten der Infektionsprävention Stellung zu beziehen.

Die Weltgesundheitsorganisation (World Health Organisation, WHO) veröffentlichte 2017 Leitlinien zu den Kernkomponenten der Infektionsprävention [12]. Diese Leitlinien wurden nach den Anforderungen des WHO- 
Handbuchs für die Leitlinienentwicklung erarbeitet [13] und waren eine Weiterentwicklung der evidenzbasierten Leitlinien der SIGHT-Gruppe, die Fachliteratur mit Publikationsdatum von 1996-2012 berücksichtigte [14]. Durch die WHO-Arbeitsgruppe wurden entsprechende PICO-Fragen formuliert, und es wurde ein weiteres systematisches Review durchgeführt, das Studien bis 2015 hinzuzog [15]. Zur Bewertung derStudien wurden die für die CochraneEPOC-Reviews entwickelten Kriterien angewendet [16].

Die deutsche Sepsisleitlinienkommission bewertete die WHO-Kernkomponenten erneut in Bezug auf deren Adaptation. Dabei wurden 6 der 8 Komponenten als Expertenkonsens eingestuft. Für die übrigen 2 Empfehlungen wurde die entsprechende Literatur angeführt. Zusätzlich wurde unter Anwendung der Suchkriterien des WHO-Reviews eine Literatursuche und Bewertung für den Zeitraum 2016-2017 für diese beiden Empfehlungen durchgeführt. Danach sind unserer Kenntnis nach keine neuen Studien zu diesem Thema erschienen, die zu einer Veränderung der Formulierung der beiden WHO-Kernkomponenten führen würden. Eine weitere Empfehlung zur Implementierung eines AntibioticStewardship(ABS)-Programms wurde in die Sepsisleitlinie integriert.

\section{1. | Empfehlung | 2018}

\section{- Expertenkonsens \\ - Wir empfehlen, dass ein Infektions- präventionsprogramm mit einem speziell dafür ausgebildeten Team in der Einrichtung implementiert ist. \\ - WHO-Leitlinienadaptation; Abwei- chung des Empfehlungsgrads \\ - Konsensstärke: $100 \%$}

Begründung. Ein Infektionspräventionsprogramm formuliert klare Präventionsziele auf der Basis der lokalen Epidemiologie, setzt Prioritäten entsprechend der lokalen Risikobeurteilung und ergreift Maßnahmen zur Prävention von nosokomialen Infektionen und zur Verhinderung der Ausbreitung von Antibiotikaresistenzen. In Deutschland werden die Existenz eines solchen Programms und die Beschäftigung von speziellem Fachpersonal zu dessen Umsetzung durch das Infektionsschutzgesetz und die Landeshygieneverordnungen gefordert.

Die WHO leitet die Evidenz für die Effektivität dieser Forderung, die in Deutschland ohnehin gesetzlich vorgegeben ist, von 2 Studien von Haley et al. und Mermel et al. ab $[17,18]$.

\section{2. | Empfehlung | 2018}

\section{- Expertenkonsens}

- Wir empfehlen die Implementierung eines Antibiotic-Stewardship(ABS)-

Programms zur Sicherung rationaler Antibiotikaanwendung im Krankenhaus.

- Zusätzliche DSG-Leitlinienempfehlung

- Konsensstärke: $100 \%$

Begründung. In der aktuellen S3-Leitlinie „Strategien zur Sicherung rationaler Antibiotika-Anwendung im Krankenhaus" wird ein ABS-Programm gefordert [19]. Auch wird dieses in den meisten Hygieneverordnungen der Bundesländer gesetzlich vorgegeben.

\section{3. | Empfehlung | 2018}

- Wir empfehlen, Fortbildungen auf dem Gebiet der Infektionsprävention für alle Mitarbeiter in allen Krankenhäusern zu etablieren. Diese Fortbildungen sollten ein Training am Krankenbett bzw. ein Simulationstraining einschließen.

- WHO-Leitlinienadaptation

- Konsensstärke: $93 \%$

- Empfehlungsgrad: stark

- Evidenzgrad: moderat

Begründung. Die Ergebnisse der in den WHO-Leitlinie zitierten 15 Studien wurden für die Formulierung der Empfehlung berücksichtigt [12]. Demnach können Fortbildungen mit einem praxisorientierten „Hands-on“-Ansatz und unter Berücksichtigung der individuellen Erfahrungen zu einer Reduktion von noso- komialen Infektionen und einer verbesserten Händehygienecompliance führen.

In Deutschland wird durch die Hygieneverordnungen der Länder gefordert, dass mindestens einmal pro Jahr eine Schulung zur Infektionsprävention durchzuführen ist.

4. | Empfehlung | 2018

\section{- Expertenkonsens}

- Wir empfehlen, eine stations- bzw. einrichtungsbezogene Surveillance von nosokomialen Infektionen (einschließlich multiresistenten Erregern) mit zeitnahem Feedback der Ergebnisse und im Kontext von nationalen Surveillance-Netzwerken durchzuführen.

- WHO-Leitlinienadaptation; Abweichung des Empfehlungsgrads

- Konsensstärke: $100 \%$

Begründung. Surveillance-Systeme können dabei helfen, Schwachstellen in Hygienekonzepten $\mathrm{zu}$ identifizieren und entsprechend zu intervenieren. Deshalb sind sie eine essenzielle Komponente der Infektionskontrollprogramme.

Die in den WHO-Empfehlungen angeführten 13 Studien zur Infektions-Surveillance (11 nichtkontrollierte „Beforeafter“-Studien, eine ,interrupted time series" und eine qualitative Studie) haben gezeigt, dass ein krankenhausbasiertes Surveillance-System zu einer Reduktion der nosokomialen Infektionen beitragen kann, besonders wenn es mit einem nationalen Surveillance-Netzwerk assoziiert ist [12].

In Deutschland wird durch das Infektionsschutzgesetz die Surveillance von nosokomialen Infektionen entsprechend den Vorgaben des Robert Koch-Instituts gefordert [20].

\section{5. | Empfehlung | 2018}

- Wir empfehlen, multimodale Strategien zur Implementierung von Infektionspräventionsmaßnahmen einzusetzen.

- WHO-Leitlinienadaptation

- Konsensstärke: $100 \%$ 
- Empfehlungsgrad: stark

- Evidenzgrad: niedrig

Begründung. Innerhalb von multimodalen Strategien werden verschiedene Interventionen ( 3 oder mehr) zu einem Gesamtkonzept verbunden, z. B. in Checklisten, die durch multidisziplinäre Teams entsprechend den lokalen Bedingungen entwickelt werden. Sie sind insbesondere bei der Verbesserung der Händehygienecompliance sowie der Reduktion von mit zentralem Venenkatheter (ZVK) assoziierten Blutstrominfektionen und beatmungsassoziierten Pneumonien relevant, aber auch bei der Senkung der Zahl von Infektionen mit methicillinresistentem Staphylococcus aureus (MRSA) und Clostridium difficile (C. difficile). Die Evidenz wurde entsprechend der in den evidenzbasierten Empfehlungen der WHO angeführten 44 Studien evaluiert [12].

Insgesamt wurde die Evidenz als niedrig beurteilt, da zitierte Studien und Studiendesigns ein mittleres bis hohes Biasrisiko aufzeigten. Auf Basis dieser Evidenz sprach sich die Leitliniengruppe der WHO für eine starke Empfehlung aus, multimodale Strategien zur Infektionsprävention einzusetzen.

In Deutschland kann die Evidenz für verschiedene Einzelmaßnahmen der multimodalen Strategien den jeweiligen KRINKO-Empfehlungen entnommen werden.

\section{6. | Empfehlung | 2018}

\section{- Expertenkonsens}

- Wir empfehlen, regelmäßig die Einhaltung von Standards zur Infektionsprävention zu überprüfen und Feedback an alle beobachteten Einheiten zu geben.

- WHO-Leitlinienadaptation; Abweichung des Empfehlungsgrads

- Konsensstärke: $100 \%$

Begründung. Die Evaluation der Evidenz aus 6 Studien durch die WHO zeigt, dass reguläres Monitoring inklusive Feedback effektiv ist, um die Compliance mit bestimmten Pflegemaßnahmen (z. B. Händehygiene) zu steigern und die noso- komialen Infektionsraten $\mathrm{zu}$ reduzieren [12].

In Deutschland gehört es zum Aufgabenspektrum der Hygienefachkräfte, solche Überprüfungen (Audits) vorzunehmen.

\section{7.| Empfehlung | 2018}

\section{- Expertenkonsens}

- Wir empfehlen, dass die Bettenbelegung nicht die Standardkapazität der Einrichtung übersteigen sollte und die Personalausstattung adäquat entsprechend der mit den Patienten verbundenen Arbeitsbelastung festgelegt werden sollte.

- WHO-Leitlinienadaptation; Abweichung des Empfehlungsgrads

- Konsensstärke: $100 \%$

Begründung. Es gibt hinreichend Studien, die eine Assoziation zwischen (niedrigem) Personalschlüssel und (hohen) nosokomialen Infektionsraten gezeigt haben. Für Deutschland wurde dies u. a. in der Studie von Schwab et al. [21] untersucht und dargelegt.

Es ist allerdings nicht möglich, auf Basis der Studien einen Schwellenwert für eine Mindestpersonalausstattung anzugeben. Hintergrund dafür sind die unterschiedliche Risikostruktur der Patienten in den verschiedenen Studien und die unterschiedlichen Methoden zur Bestimmung der Personalausstattung. Dadurch sind die Daten der Studien nicht im Sinne einer Metaanalyse verwendbar.

Die WHO-Leitlinie sprach anhand von 19 Studien eine starke Zustimmung bei sehr geringer Beweisqualität aus, dass eine über die Standardkapazität der Einrichtung hinausgehende Bettenbelegung sowie ein unzureichender Personalbestand im Gesundheitswesen mit dem erhöhten Risiko einer nosokomialen Infektion verbunden sind.

\section{8. | Empfehlung | 2018}

- Expertenkonsens

- Wir empfehlen, die notwendigen Materialien für die Händehygiene unmittelbar am Behandlungsort zur Verfügung zu stellen.
- WHO-Leitlinienadaptation; Abweichung des Empfehlungsgrads

- Konsensstärke: $100 \%$

Begründung. In verschiedenen Studien konnte gezeigt werden, dass die Händehygienecompliance signifikant gesteigert werden konnte, wenn die notwendigen Händedesinfektionsmittelspender in unmittelbarer Nähe zum Patientenbett zugänglich waren [22-27]. In Deutschland wird das auch durch die entsprechende KRINKO-Empfehlung gefordert [28].

\section{Impfungen}

In den Empfehlungen der SSC werden Impfungen nicht separat erwähnt. $\mathrm{Da}$ Impfungen jedoch eine wesentliche Präventionsmaßnahme zur Reduktion von Mortalität und Morbidität schwerer Infektionen und damit der Sepsis darstellen, ist aus Sicht der Leitlinienkommission ein Verweis erforderlich.

\section{1. | Empfehlung | 2018}

\section{- Expertenkonsens}

- Wir empfehlen die Umsetzung der Empfehlungen der Ständigen Impfkommission (STIKO) am Robert KochInstitut. Insbesondere die Impfungen gegen Influenza und Pneumokokken sowie die Impfungen gegen Meningokokken und Haemophilus influenzae Typ b können dazu beitragen, die Inzidenz der Sepsis zu reduzieren. Auch die jährliche Impfung von medizinischem Personal gegen Influenza trägt dazu bei, Patienten vor nosokomialer Influenza zu schützen.

- Zusätzliche DSG-Leitlinienempfehlung

- Konsensstärke bei Doppelabstimmung: $90 \%$

Begründung. Die Leitlinienkommission unterstützt die Empfehlungen der Ständigen Impfkommission (STIKO) am Robert Koch-Institut. Insbesondere die Impfungen gegen Influenza und Pneumokokken sowie die Impfungen gegen Meningokokken und Haemophilus in- 
fluenzae Typ b können dazu beitragen, die Inzidenz der Sepsis zu reduzieren. Insbesondere wird auf die in diesen Empfehlungen genannte altersunabhängige Indikationsimpfungen bei Patienten mit erhöhter Gefährdung für die o.g. Erreger verwiesen, dies gilt insbesondere für Patienten mit funktioneller oder anatomischer Asplenie.

Um vulnerable Patienten vor nosokomialer Influenza, die mit einer relevanten Letalität assoziiert ist, zu schützen, sollte sich medizinisches Personal jährlich zu Beginn der Saison mit den jeweils empfohlenen Vakzinen impfen lassen.

Die ambulant erworbene und die nosokomial erworbene Pneumonie sind der häufigste Fokus für Sepsis und septischen Schock [29]. Der häufigste virale und der häufigste bakterielle Erreger der ambulant erworbenen Pneumonie, Influenzavirus und Streptococcus pneumoniae (Pneumokokken), sind impfpräventabel. Beide Erreger können gemeinsam als Mischinfektion bzw. Pneumokokken als bakterielle Superinfektion auftreten. Bei diesen Infektionen steigt die Letalität gegenüber der Monoinfektion deutlich an [30]. Beide Erreger verursachen auch einen wesentlichen Teil der nosokomialen Pneumonie. Die zunehmende Erkenntnis, dass nosokomiale Influenza ein relevantes Problem darstellt, findet sich auch in der aktuellen Leitlinie zur nosokomialen Pneumonie [31, 683, 684]. Eine Erhebung an einem deutschen Universitätsklinikum in der Saison 2014/15 zeigte, dass $35 \%$ aller Nachweise nosokomialen Ursprungs waren; die Letalität betrug $10 \%$ [32]. Daher unterstützt die Leitlinienkommission nachdrücklich die Empfehlung zur jährlichen Impfung des medizinischen Personals.

\section{A. Initiale hämodynamische Stabilisierung ${ }^{1}$}

\section{A.1 | Empfehlung | 2018}

\section{- Expertenkonsens \\ - Sepsis und septischer Schock sind medizinische Notfälle. Wir empfeh-}

1 Der Begriff "initial resuscitation“ aus der SSC-Leitlinie wird im Kapitel A mit hämodynamischerStabilisierung übersetzt. len, dass mit der Behandlung und der hämodynamischen Stabilisierung unverzüglich begonnen wird.

- SSC-Leitlinienadaptation

- Konsensstärke: $100 \%$

Begründung. Eine frühzeitige wirksame Flüssigkeitstherapie ist von entscheidender Bedeutung für die Stabilisierung einer sepsisinduzierten Gewebehypoperfusion oder eines septischen Schocks. Eine sepsisinduzierte Hypoperfusion kann sich durch eine akute Organdysfunktion und/oder eine Verringerung des Blutdrucks sowie eine Erhöhung des Serumlaktats manifestieren. Frühere Varianten dieser Leitlinien enthielten die Empfehlung einer protokollbasierten quantitativen Flüssigkeitstherapie, die auch unter der Bezeichnung frühzeitige zielgerichtete Therapie („early goal-directed therapy“, EGDT) bekannt ist, basierend auf dem von Rivers publizierten Protokoll [33]. Diese Empfehlung beschrieb die Nutzung einer Reihe von „Zielen“, zu denen der zentralvenöse Druck („central venous pressure“, CVP) und die zentralvenöse Sauerstoffsättigung („central venous oxygen saturation“, $\mathrm{S}_{\mathrm{cv}} \mathrm{O}_{2}$ ) gehörten. Dieser Ansatz wurde nun hinterfragt, nachdem in 3 aufeinanderfolgenden großen multizentrischen randomisierten kontrollierten Studie („randomized controlled trials“, RCT) kein Nachweis einer Mortalitätsreduktion erfolgt ist [34-36]. Mit den Interventionsstrategien im Sinne einer EGDT war jedoch auch keine Schädigung verbunden. Somit kann die Nutzung der bisherigen Ziele immer noch als sicher beurteilt werden, sodass diese berücksichtigt werden können.

Es ist darauf hinzuweisen, dass die aktuelleren Studien weniger schwer erkrankte Patienten einschlossen (geringere Baselinelaktatwerte, $\mathrm{S}_{\mathrm{cv}} \mathrm{O}_{2}$ bei der Aufnahme entsprechend dem Zielwert oder höher als der Zielwert, geringere Mortalität in der Kontrollgruppe). Dieses Protokoll kann aufgrund der aktuellen Evidenzbasis nicht empfohlen werden. Trotzdem benötigen Krankenhausärzte eine Orientierungshilfe für den Umgang mit oben genannter Patientengruppe, bei der eine signifikante Mortalität und Mor- biditätbesteht. Wir empfehlen daher, dass diese Patienten als medizinischer Notfall angesehen werden, der eine sofortige $\mathrm{Be}$ urteilung und Behandlung erforderlich macht.

\section{A.2a | Empfehlung | 2018}

- Wir empfehlen, dass zur hämodynamischen Stabilisierung bei Patienten mit sepsisinduzierter Hypoperfusion eine intravenöse kristalloide Lösung innerhalb der ersten $3 \mathrm{~h}$ verabreicht wird.

- SSC-Leitlinienadaptation

- Konsensstärke: $100 \%$

- Empfehlungsgrad: stark

- Evidenzgrad: niedrig

\section{A.2b | Empfehlung | 2018 \\ - Wir schlagen vor, mindestens $30 \mathrm{ml} / \mathrm{kg} \mathrm{KG}$ intravenöse kristal- loide Lösung in den ersten $3 \mathrm{~h} \mathrm{zu}$ verabreichen. \\ - SSC-Leitlinienadaptation; Abwei- chung des Empfehlungsgrads \\ - Konsensstärke: $100 \%$ \\ - Empfehlungsgrad: schwach \\ - Evidenzgrad: niedrig}

Begründung. Als Teil dieser Notfallbehandlung empfehlen wir, bei der initialen Flüssigkeitstherapie mit $30 \mathrm{ml} / \mathrm{kg} \mathrm{KG}$ kristalloider Lösung innerhalb der ersten $3 \mathrm{~h} \mathrm{zu}$ beginnen. Dieses festgelegte Flüssigkeitsvolumen ermöglicht dem Kliniker, die Flüssigkeitstherapie zu initiieren, während er genauere Informationen über den Patienten einholt und auf präzisere Messergebnisse des hämodynamischen Status wartet. Obwohl es in der vorhandenen Literatur nur wenige kontrollierte Daten gibt, die dieses Flüssigkeitsvolumen unterstützen, haben aktuelle interventionelle Studien dies als die übliche Praxis in den frühen Stadien der Flüssigkeitstherapie beschrieben. Die Evidenz aus Beobachtungsstudien unterstützt diese Praxis [37, 38]. Das durchschnittliche Flüssigkeitsvolumen vor der Randomisierung, das in der PROCESSund der ARISE-Studie verabreicht wurde, betrug etwa $30 \mathrm{ml} / \mathrm{kg} \mathrm{KG}$, und in der 
PROMISE-Studie etwa 21 [34-36]. Viele Patienten werden mehr Flüssigkeit benötigen und für diese Gruppe befürworten wir, dass mehr Flüssigkeit unter einem Monitoring funktionaler hämodynamischer Messungen verabreicht wird.

Abweichend von der SSC-Leitlinie wird aufgrund einer aktualisierten Beurteilung expertenbeigesteuerter Literatur mit neueren Untersuchungen hochwertiger Qualität in Bezug auf die Risk-of-Bias-Bewertung nur eine schwache Empfehlung für eine schematische Mindestinfusionsmenge ausgesprochen [39-42]. In prospektiven bzw. konsekutiven Beobachtungsstudien an Kohorten mit Sepsis und septischem Schock wurde ein positiver Effekt einer zeitnahen ( $\leq 30 \mathrm{~min}$ vs. $31-120 \mathrm{~min}$ vs. $>120 \mathrm{~min}$ ) Initiierung einer kristalloiden Flüssigkeitstherapie auf die Krankenhaussterblichkeit entweder alleinig [42] oder in Zusammenhang mit einem Bündel von Maßnahmen berichtet [39]. In einer ebenfalls neueren retrospektiven Studie an 49.331 Patienten mit Sepsis und septischem Schock konnte kein Überlebensvorteil bei Komplettierung des kristalloiden Flüssigkeitsbolus von $30 \mathrm{ml} / \mathrm{kg} \mathrm{KG}$ innerhalb der ersten $12 \mathrm{~h}$ nach Protokollinitiierung gezeigt werden [40]. Eine neuere prospektive Studie bei Patienten mit Sepsis und Hypotension in einem Entwicklungsland zeigte eine erhöhte Krankenhaussterblichkeit $(48,1 \%$ vs. $33,0 \%)$ nach protokollbasierter Infusion von 3,51 vs. 2,01 („usual care"/Standardtherapie) kristalloider Infusionslösung innerhalb von $6 \mathrm{~h}$ [39]. In der Gesamtbetrachtung der vorliegenden Studien wurde dies so interpretiert, dass eine zeitnahe Initiierung der Flüssigkeitstherapie von Vorteil ist, jedoch nicht die schematische Verabreichung des vollständigen $30 \mathrm{ml} / \mathrm{kg} \mathrm{KG-Flüssig-}$ keitsbolus innerhalb der ersten $3 \mathrm{~h}$.

\section{A.3 | Empfehlung | 2018}

\section{- Expertenkonsens}

- Wir empfehlen, dass zusätzliche Flüssigkeitsgaben nach dem initialen Flüssigkeitsbolus im Rahmen der weiteren initialen Stabilisierung durch häufig wiederholte Kontrollen des hämodynamischen Status begleitet werden.

- Wir empfehlen ferner, eine fortgesetzte Flüssigkeitstherapie nur in Erwägung zu ziehen, wenn weiterhin Zeichen einer Hypoperfusion vorliegen.

- Bemerkungen: Eine wiederholte Kontrolle sollte eine gründliche klinische Untersuchung und Bewertung der verfügbaren physiologischen Variablen (Herzfrequenz, Blutdruck, arterielle und zentralvenöse Sauerstoffsättigung, Atemfrequenz, Temperatur, Urinausscheidung und sonstige Variablen je nach Verfügbarkeit) beinhalten sowie weitere nichtinvasive und invasive Überwachungsmaßnahmen, sofern derartige Maßnahmen zur Verfügung stehen.

- SSC-Leitlinienadaptation und SSCLeitlinienmodifikation

- Konsensstärke: $100 \%$

Begründung. Eines der wichtigsten Prinzipien, das in Bezug auf die komplexe Behandlungsplanung dieser Patienten berücksichtigt werden muss, ist die Notwendigkeit einer detaillierten initialen Beurteilung und von kontinuierlich $\mathrm{zu}$ wiederholenden Kontrollen hinsichtlich des Ansprechens auf die Behandlung. Diese Beurteilung sollte mit einer gründlichen klinischen Untersuchung und Evaluierung der verfügbaren physiologischen Variablen beginnen, die der Beschreibung des klinischen Zustands des Patienten dienen (Herzfrequenz, Blutdruck, arterielle Sauerstoffsättigung, Atemfrequenz, Temperatur, Urinausscheidung u.a. je nach Verfügbarkeit).

Ergänzend zu der SSC-Leitlinie wird neben der arteriellen auch die zentralvenöse Sauerstoffsättigung als physiologische Variable aufgeführt, da ein deutlich erniedrigter Wert ein wichtiges Zeichen eines gestörten Verhältnisses zwischen $\mathrm{O}_{2}$-Angebot und $\mathrm{O}_{2}$-Verbrauch darstellt und ein solcher Befund bei der Verlaufsbeurteilung berücksichtigt werden sollte. So wiesen Patienten, bei denen nach initial erniedrigter $\mathrm{S}_{\mathrm{cv}} \mathrm{O}_{2}(<70 \%)$ innerhalb der ersten $6 \mathrm{~h}$ ein Anstieg der $\mathrm{S}_{\mathrm{cv}} \mathrm{O}_{2}$ auf $>70 \%$ gemessen wurde, eine deutlich niedrigere Mortalität auf als Patienten, die initial und auch während der ersten $6 \mathrm{~h}$ eine erniedrigte $\mathrm{S}_{\mathrm{cv}} \mathrm{O}_{2}$ aufwiesen [43].

\section{A.4 | Empfehlung | 2018}

\section{- Expertenkonsens \\ - Wir empfehlen eine zusätzliche hä- modynamische Beurteilung (wie z. B. die echokardiographische Beurtei- lung der Herzfunktion) zur Ermittlung der Art des Schocks, falls die klini- sche Untersuchung nicht zu einer eindeutigen Diagnose führt. \\ - SSC-Leitlinienadaptation und SSC- Leitlinienmodifikation \\ - Konsensstärke: $100 \%$}

Begründung. Ergänzend zur SSC-Leitlinie wird (in Übereinstimmung mit der S3-Leitlinie zur intravasalen Volumentherapie beim Erwachsenen [44]) die Echokardiographie als Methode zur Beurteilung der Herzfunktion explizit aufgeführt. In den letzten Jahren ist die Echokardiographie für viele Krankenhausärzte verfügbar geworden, die eine genauere Analyse der Ursachen für die hämodynamischen Probleme ermöglicht [45]. Mittels der Echokardiographie kann beim Patienten mit unklarer hämodynamischer Instabilität eine Vielzahl von relevanten Differenzialdiagnosen (Perikarderguss und -tamponade, akute Rechtsherzbelastung als Hinweis für eine Lungenarterienembolie [LAE], eingeschränkte Pumpfunktion, Klappenvitium etc.) ausgeschlossen werden. Insbesondere beim kardiogenen Schock sind transthorakale Echokardiographie (TTE) und transösophageale Echokardiographie (TEE) essenzieller Bestandteil der Diagnostik. Die American Heart Association wertet den Einsatz der transthorakalen Echokardiographie bei Patienten mit Hypotension oder hämodynamischer Instabilität mit unklarer Ursache als „appropriate“ mit höchstem Empfehlungsgrad [46].

\section{A.5 | Empfehlung | 2018}

\section{- Empfehlungsgrad: schwach \\ - Evidenzgrad: niedrig \\ - Wir schlagen vor, dass bevorzugt dynamische gegenüber statischen}


Variablen verwendet werden, um das Ansprechen auf eine Flüssigkeitstherapie vorherzusagen, sofern diese zur Verfügung stehen.

- SSC-Leitlinienadaptation

- Konsensstärke: $79 \%$

Begründung. Die ausschließliche Nutzung des zentralvenösen Drucks allein zur Steuerung der Flüssigkeitstherapie kann nicht länger empfohlen werden [45], weil die Fähigkeit zur Prognose der Volumenreagibilität limitiert ist, wenn der zentrale Venendruck (ZVD) innerhalb eines relativ normalen Wertebereichs $(8-12 \mathrm{~mm} \mathrm{Hg})$ liegt [47]. Das Gleiche gilt für andere statische Messungen des rechten oder linken Vorhofdrucks oder -volumens. Dynamische Messungen zur Beurteilung, ob ein Patient zusätzliche Flüssigkeit benötigt, wurden mit dem Ziel vorgeschlagen, das Flüssigkeitsmanagement $\mathrm{zu}$ verbessern. Diese haben eine bessere diagnostische Genauigkeit bei der Prädiktion von Patienten gezeigt, die wahrscheinlich auf eine Flüssigkeitsbolusgabe mit einem erhöhten Schlagvolumen ansprechen werden. Diese Techniken umfassen die passive Beinhochlagerung, die probatorische Zufuhr definierter Flüssigkeitsboli unter gleichzeitigen Schlagvolumenmessungen oder Variationen des systolischen Drucks, des Pulsdrucks oder des Schlagvolumens auf Änderungen des intrathorakalen Drucks unter invasiver Beatmung [48]. Ein Review von 5 Studien zur Nutzung einer Pulsdruckvariation (PPV) zur Prognose des Ansprechens auf eine Flüssigkeitstherapie bei Patienten mit Sepsis oder septischem Schock ergab eine Sensitivität von 0,72 (95\%-KI 0,61-0,81) und eine Spezifität von 0,91 (95\%-KI 0,83-0,95); die Evidenzqualität war niedrig, was auf Ungenauigkeiten und einen potenziellen Bias zurückzuführen ist [48]. Eine aktuelle multizentrische Studie demonstrierte einen begrenzten Nutzung eines hämodynamischen Monitorings während der Flüssigkeitsverabreichung auf der ITS. Obwohl es an Daten bezüglich der Nutzung dieser Monitore auf Notfallstationen mangelt, könnte die Verfügbarkeit dieser Geräte und eine universelle Anwendbarkeit der Para- meter die routinemäßige Nutzung von dynamischen Indizes beeinflussen [45, 49].

Ergänzend zur SSC-Leitlinie schlagen wir mit Bezug auf die S3-Leitlinie Intravasale Volumentherapie [50] dynamische Variablen vor. Diese umfasst z. B. die „pulse pressure variation“ (PPV), die Schlagvolumenvariation, die systolische Druckvariation, die Vena-cavainferior-Variabilität, die passive Beinhochlagerung oder die probatorische Zufuhr definierter Flüssigkeitsboli unter gleichzeitigen Schlagvolumenmessungen. Voraussetzungen für die valide Anwendung der PPV zur Prädiktion der Volumenreagibilität:

- maschinelle Beatmung,

- keine Spontanatmungsanteile,

- keine Arrhythmie,

- Tidalvolumen $\geq 8 \mathrm{ml} / \mathrm{kg} \mathrm{KG}$ Idealgewicht,

- Herzfrequenz/Atemfrequenz >3,6,

- totale respiratorische Compliance $>30 \mathrm{ml} / \mathrm{cm} \mathrm{H}_{2} \mathrm{O}$,

- ausreichende Rechtsherzfunktion (gemäß Echokardiographie).

\section{A.6 | Empfehlung | 2018}

\section{- Expertenkonsens}

- Wir empfehlen, eine fortgesetzte Flüssigkeitstherapie nur in Erwägung zu ziehen, wenn Zeichen einer Hypoperfusion vorliegen.

- Zusätzliche DSG-Leitlinienempfehlung

- Konsensstärke: $100 \%$

Begründung. Ergänzend wird ferner darauf hingewiesen, dass eine positive Vorhersage der Volumenreagibilität ohne weitere Zeichen der Hypoperfusion allein keine Indikation zur Flüssigkeitstherapie begründet. In einer retrospektiven Kohortenstudie an 405 Patienten mit Sepsis und septischem Schock war eine Flüssigkeitsüberladung in den ersten Tagen mit einer erhöhten Krankenhaussterblichkeit assoziiert [51]. In einem retrospektiven Review des VASST-Trials korrelierte eine positive Flüssigkeitsbilanz bei Patienten mit septischem Schock nach $12 \mathrm{~h}$ bzw. 4 Tagen mit einer erhöhten 28-TageSterblichkeit [52]. In einer prospektiven
Studie an 173 konsekutiven Patienten mit Sepsis in den ersten 7 Tagen konnte eine positive Flüssigkeitsbilanz als unabhängiger prognostischer Faktor für eine erhöhte Krankenhaussterblichkeit ermittelt werden [53]. Eine flüssigkeitsrestriktive Therapie nach dem initialen Flüssigkeitsbolus hatte in einer prospektiven Studie [54] und nachfolgender Post-hoc-Analyse [55] von 151 Patienten keine negativen Auswirkungen auf den Laktatverlauf, den Noradrenalinbedarf, die Urinausscheidung in den ersten $24 \mathrm{~h}$ bzw. die Verschlechterung einer akuten Nierenschädigung. Allerdings gab es keinen signifikanten Effekt auf die 90Tage-Sterblichkeit oder auf ischämische Ereignisse während der Intensivtherapie.

\section{A.7| Empfehlung | 2018}

\section{- Wir schlagen bei Patienten mit sep- tischem Schock, die Vasopressoren benötigen, für den mittleren arteriel- len Druck (MAP) einen anfänglichen Zielwert in Höhe von $65 \mathrm{~mm} \mathrm{Hg}$ vor. - SSC-Leitlinienadaptation; Abwei- chung des Empfehlungsgrads \\ - Konsensstärke: $100 \%$ \\ - Empfehlungsgrad: schwach \\ - Evidenzgrad: niedrig}

Begründung. Mittlerer arterieller Druck („mean arterial pressure“, MAP) ist der Druck, der die Gewebeperfusion steuert. Obwohl die Perfusion der wichtigsten Organe wie des Gehirns oder der Niere vor einer systemischen Hypotonie durch eine Autoregulation der regionalen Perfusion aufrechterhalten werden kann, wird die Gewebeperfusion unterhalb eines MAP-Schwellenwerts linear von dem arteriellen Druck abhängig. In einer monozentrischen Studie [56] erhöhte die Norepinephrindosistitration von einem MAP von $65 \mathrm{~mm} \mathrm{Hg}$ auf 75 und $85 \mathrm{~mm} \mathrm{Hg}$ den Herzindex (von $4,7 \pm 0,5$ auf $5,5 \pm 0,61 / \mathrm{min} / \mathrm{m}^{2}$ ), sie führte aber nicht zu einer Änderung des Harnflusses, der arteriellen Laktatwerte, der Sauerstoffversorgung und des Sauerstoffverbrauchs, des $\mathrm{PCO}_{2}$ der Magenschleimhaut, der RBC-Geschwindigkeit oder der Kapillarströmung der Haut. Eine weitere monozentrische Stu- 
die [57] verglich eine Dosistitration beim mit Norepinephrin behandelten septischen Schock zur Aufrechterhaltung eines MAP von $65 \mathrm{~mm} \mathrm{Hg}$ gegenüber dem Erreichen von $85 \mathrm{~mm} \mathrm{Hg}$. In dieser Studie erhöhte ein höherer MAP den Herzindex von 4,8 $(3,8-6,0)$ auf 5,8 $(4,3-6,9) \mathrm{l} / \mathrm{min}$ und $\mathrm{m}^{2}$, führte jedoch zu keiner Änderung der Nierenfunktion, der arteriellen Laktatwerte oder des Sauerstoffverbrauchs. Eine dritte monozentrische Studie [58] wies eine Verbesserung der Mikrozirkulation nach, entsprechend der Beurteilung anhand der sublingualen Gefäßdichte und der Steigung der Sauerstoffsättigungskurve am Daumenballen nach einem Okklusionstest, indem eine Titration von Norepinephrin auf einen MAP von $85 \mathrm{~mm} \mathrm{Hg}$ im Vergleich zu $65 \mathrm{~mm} \mathrm{Hg} \mathrm{er-}$ folgte. Nur eine multizentrische Studie, bei der die Norepinephrindosistitration zum Erreichen eines MAP von $65 \mathrm{~mm} \mathrm{Hg}$ vs. $85 \mathrm{~mm} \mathrm{Hg}$ verglichen wurde, führte die Mortalität als primäres Ergebnis auf [59]. Es lag kein signifikanter Unterschied bezüglich der Mortalität nach Ablauf von 28 Tagen $36,6 \%$ in der Gruppe mit hohem Zielwert und 34,0\% in der Gruppe mit niedrigem Zielwert) oder 90 Tagen $(43,8 \%$ in der Gruppe mit hohem Zielwert und $42,3 \%$ in der Gruppe mit niedrigem Zielwert) vor. Die Anvisierung eines MAP von $85 \mathrm{~mm} \mathrm{Hg}$ führte $\mathrm{zu}$ einem signifikant erhöhten Risiko für Arrhythmien, wobei in einer Untergruppe von Patienten mit vorbestehender arterieller Hypertonie ein reduzierter Bedarf für eine Nierenersatztherapie („renal replacement therapy“, RRT) bei diesem höheren MAP bestand. Eine aktuelle Pilotstudie an 118 Patienten mit septischem Schock [60] wies darauf hin, dass bei der Untergruppe der Patienten, die älter als 75 Jahre waren, die Mortalität reduziert war, wenn ein MAP von $60-65 \mathrm{~mm} \mathrm{Hg}$ im Vergleich zu $75-80 \mathrm{~mm} \mathrm{Hg}$ anvisiert wurde. Die Evidenzqualität war moderat, was auf potenziellen Bias zurückzuführen ist (große Konfidenzintervalle). Daraus resultierte, dass die positiven Effekte eines Ziel-MAP von $65 \mathrm{~mm} \mathrm{Hg}$ (geringeres Risiko für Vorhofflimmern, geringere Vasopressordosis und ähnliche Mortalität) zu einer starken Empfehlung führten, sodass ein initialer MAP-Zielwert von $65 \mathrm{~mm} \mathrm{Hg}$ gegenüber höheren MAP-Zielwerten favorisiert wurde. Sobald mehr Erkenntnisse zum Zustand des jeweiligen Patienten gewonnen werden können, sollte dieser Zielwert an die betreffenden Umstände angepasst werden.

Aufgrund einer abweichenden Beurteilung der vorliegenden Literatur wird nur eine schwache Empfehlung für den MAP-Zielwert von $65 \mathrm{~mm} \mathrm{Hg}$ ausgesprochen. Die multizentrische Studie von Asfar et al. [59] zielte darauf ab, Zielblutdruckwerte von $65-70 \mathrm{~mm} \mathrm{Hg}$ bzw. 80-85 mm Hg zu vergleichen. Tatsächlich verglich die Studie Zielblutdruckwerte von $75 \mathrm{~mm} \mathrm{Hg}$ bzw. $85 \mathrm{~mm} \mathrm{Hg}$. Die aktuelle Pilotstudie von Lamontagne et al. [60] verfehlte bei Einhaltung des oberen Zielbereichs von $75-80 \mathrm{~mm} \mathrm{Hg}$ den unteren Zielbereich von $60-65 \mathrm{~mm} \mathrm{Hg}$ und verglich $70 \mathrm{~mm} \mathrm{Hg}$ mit $79 \mathrm{~mm} \mathrm{Hg}$. Somit liegen aktuell keine ausreichenden klinischen Daten vor, die den Zielwert von $65 \mathrm{~mm} \mathrm{Hg}$ für den MAP im Vergleich zu anderen Zielwerten vergleicht, sodass nur eine schwache Empfehlung ausgesprochen werden kann.

\section{A.8 | Empfehlung | 2018}

\section{- Wir schlagen vor, bei Patienten, bei denen erhöhte Laktatwerte infolge einer Gewebehypoperfusion vorliegen, die hämodynamische Stabilisierung mit dem Ziel einer Normalisierung der Laktatwerte zu steuern. \\ - SSC-Leitlinienadaptation \\ - Konsensstärke: $100 \%$ \\ - Empfehlungsgrad: schwach \\ - Evidenzgrad: niedrig}

Begründung. Das Serumlaktat ist kein direkter Maßstab für die Gewebeperfusion [61]. Erhöhungen der Serumlaktatwerte können durch eine Gewebehypoxie, eine beschleunigte aerobe Glykolyse durch übermäßige $\beta$-adrenerge Stimulation oder andere Ursachen bedingt sein (z. B. Leberversagen). Unabhängig von der Ursache sind jedoch erhöhte Laktatwerte mit einem schlechteren Outcome verbunden [62]. Da es sich bei Laktat um einen Standardlabortest handelt, kann es als objektiveres Surrogat für die Gewebeperfusion im Vergleich zur körperlichen Untersuchung oder der Urinausscheidung dienen. Fünf RCT (647 Patienten) haben die laktatgesteuerte Flüssigkeitstherapie bei Patienten mit septischem Schock evaluiert [63-67]. Eine signifikante Reduzierung der Mortalität wurde bei der laktatgesteuerten Flüssigkeitstherapie im Vergleich zur Flüssigkeitstherapie ohne Laktatüberwachung beobachtet (RR 0,67; 95\%-KI 0,53-0,84; niedrige Evidenz). Es wurden keine Nachweise in Bezug auf einen Unterschied bei der Aufenthaltsdauer auf der ITS (LOS) erbracht (Mittelwertdifferenz $-1,51$ Tage; $95 \%-\mathrm{KI}-3,65$ bis 0,62; niedrige Evidenz). Zwei andere Metaanalysen mit 647 Patienten, die in diese Studien einbezogen wurden, demonstrierten eine mittlere Evidenzqualität im Hinblick auf eine Reduzierung der Mortalität, wenn eine frühzeitige Laktatclearancestrategie genutzt wurde, verglichen mit einer Standardtherapie (nicht spezifiziert) oder mit einer $\mathrm{S}_{\mathrm{cv}} \mathrm{O}_{2-}$ Normalisierungsstrategie $[68,69]$.

\section{B. Leitlinienimplementierung}

\section{B.1 | Empfehlung | 2018}

\section{- Expertenkonsens \\ - Wir empfehlen Krankenhäusern, Pro- gramme zur Implementierung der Leitlinie zu initiieren und umzuset- zen, einschließlich eines Screenings für Risikopatienten. - SSC-Leitlinienadaptation - Konsensstärke: $100 \%$}

Begründung. Strategien zur Qualitätssicherung können dabei helfen, das Outcome von Sepsispatienten günstig zu beeinflussen [70]. Sie fußen im Idealfall auf einer interdisziplinären Kooperation zwischen allen Fachgebieten. Erfolgreiche Programme zur Qualitätssicherung setzen eine sorgfältige Protokollentwicklung und -umsetzung voraus, einschließlich der zu evaluierenden Messwerte, der Datenerhebung und der Maßnahmen zur 
Gewährleistung kontinuierlicher Rückmeldung an die Teilnehmer [71, 72].

Strategien zur Qualitätssicherung können auf eine frühzeitige Erkennung einer Sepsis ausgerichtet sein. Dazu können geeignete Screeningmaßnahmen beitragen oder eine Optimierung des Patientenmanagements. Die frühzeitige Identifizierung von Patienten mit Sepsis ist von hoher Bedeutung, da eine unverzügliche Therapie die Überlebenschancen wesentlich verbessern und Folgeschäden reduzieren kann [37, 73-75].

Eine Metaanalyse von 50 Observationsstudien hat gezeigt, dass Programme zur Qualitätssicherung in der Versorgung von Sepsispatienten mit einer signifikant verbesserten Compliance mit den Leitlinienempfehlungen der SSC und einer geringeren Krankenhausmortalität verbunden waren (OR 0,66; $95 \%$-KI 0,61-0,72; [76]). Die bisher größte Studie, die die Beziehung zwischen der Compliance mit den SSC-Leitlinien (basierend auf den SSC-Leitlinien aus dem Jahr 2004) und der Krankenhausmortalität untersuchte, beinhaltete 29.470 Patienten in 218 Krankenhäusern in den USA, Europa und Südamerika über einen Zeitraum von 7,5 Jahren [38]. Die Krankenhausmortalität verringerte sich um $0,7 \%$ für alle 3 Monate, in denen ein Krankenhaus die SSC-Leitlinien umsetzte, was mit einem Rückgang der Aufenthaltsdauer auf der ITS um $4 \%$ für jede $10 \%$ ige Verbesserung der Compliance in Bezug auf die Empfehlungen verbunden war. Dieser Nutzen wurde zudem in einem breiten geographischen Spektrum nachgewiesen. Eine Studie an 1794 Patienten aus 62 Ländern mit schwerer Sepsis (die nunmehr als "Sepsis“ gemäß der Sepsis-3-Definition bezeichnet wird [1]) bzw. septischem Schock zeigte eine Reduzierung der Wahrscheinlichkeit, im Krankenhaus zu versterben, in Höhe von 36-40 \% im Fall einer Compliance mit den SSC-Leitlinien auf [77]. Die spezifischen einzelnen Maßnahmen, die $\mathrm{zu}$ dieser Verbesserung beitrugen, variierten allerdings signifikant zwischen den verschiedenen Studien; somit kann keine einzelne Maßnahme explizit empfohlen werden.

\section{B.2 | Empfehlung | 2018}

\section{- Expertenkonsens}

- Wir empfehlen, bei Patienten außerhalb von ITS, bei denen der Verdacht auf eine Infektion besteht, den "quick Sequential Organ Failure Assessment" (qSOFA) Score regelmäßig zu bestimmen, um Risikopatienten mit vitaler Bedrohung frühzeitig zu erkennen. (• Abb. 1, Anhang)

- Zusätzliche DSG-Leitlinienempfehlung

- Konsensstärke: $100 \%$

Begründung. Die frühzeitige Identifizierung von Patienten mit Sepsis ist von hoher Bedeutung, da eine frühzeitige Therapie das Überleben wesentlich verbessern kann. Im Gegensatz zu anderen akut lebensbedrohlichen Erkrankungen (wie z. B. dem Herzinfarkt), stehen jedoch derzeit keine diagnostischen bettseitigen Kriterien zur Verfügung, um Patienten mit Sepsis bzw. solche mit hohem Risiko für die Entwicklung einer Sepsis zuverlässig zu identifizieren. Daher hat die Sepsis-3-Arbeitsgruppe einer einfachen diagnostischen Score (qSOFA, siehe auch Kapitel „Definitionen“) entwickelt, um Patienten mit hohem Risiko für eine Sepsis zu identifizieren. Dazu analysierte die Sepsis-3-Arbeitsgruppe mehrere, sehr große Krankenhausdatenbanken von insgesamt 850.000 Patienten unter Infektionsverdacht (definiert durch eine Kombination von Antibiotikagabe und mikrobiologischer Diagnostik innerhalb eines bestimmten Zeitfensters). Eine derartig große Kohorte wurde bei den bisher verwendeten Sepsisdefinitionen nie untersucht. Ein weiterer großer Unterschied: Lediglich $10 \%$ der Patienten befanden sich zum Zeitpunkt des Einschlusses auf einer ITS.

Der „quick Sequential Organ Failure Assessment" (qSOFA) Score nutzt zur Risikoabschätzung die Variablen veränderter mentaler Status, systolischer Blutdruck $<100 \mathrm{~mm} \mathrm{Hg}$ oder eine Atemfrequenz $\geq 22 / \mathrm{min}$. Verglichen mit einem qSOFA-Score von 0 oder 1 Punkt haben Patienten mit einem qSOFA-Score von 2 bzw. 3 Punkten ein 3-fach bzw. 14-fach erhöhtes Risiko zu versterben. Die Sepsis-
3-Taskforce hat empfohlen, die Behandlung nicht zu verzögern, bis die Patienten 2 oder mehr qSOFA-Kriterien erfüllen.

Die Mehrzahl der seit Februar 2016 publizierten Validierungsstudien bestätigen die Ergebnisse der Sepsis-3-Taskforce und belegen, dass der qSOFA-Score ein geeigneter Parameter ist, um Patienten mit Infektionsverdacht außerhalb von ITS auf das Vorliegen einer Sepsis zu screenen. Weitere prospektive Studien müssen zeigen, ob eine längsschnittliche und wiederholte Anwendung des qSOFA im intraindividuellen Verlauf sinnvoll ist. Darüber hinaus wäre eine prospektive kontrollierte Studie, die den klinischen Nutzen des qSOFA im Vergleich zu einer Standardbehandlung hinsichtlich harter Outcomeparameter untersucht, von Bedeutung [1].

\section{Diagnose}

\section{C.1 | Empfehlung | 2018}

\section{- Expertenkonsens}

- Wir empfehlen, dass regelhaft geeignete Materialien für die mikrobiologische Diagnostik (einschließlich Blutkulturen) entnommen werden, bevor die antimikrobielle Therapie bei Patienten mit Verdacht auf Sepsis oder septischen Schock begonnen wird. Dies darf zu keiner wesentlichen Verzögerung in Bezug auf den Beginn der antimikrobiellen Therapie führen.

- Bemerkungen: Geeignete Materialien für die routinemäßige mikrobiologische Kulturendiagnostik umfassen immer mindestens 2 (sowohl aerobe als auch anaerobe) Blutkultursets (bestehend aus mindestens aerober und anaerober Flasche).

- SSC-Leitlinienadaptation

- Konsensstärke: $100 \%$

Begründung. Eine Inaktivierung von Mikroorganismen kann innerhalb von Minuten bis zu mehreren Stunden nach der ersten Dosis eines geeigneten antimikrobiellen Wirkstoffs erfolgen, sodass sie möglicherweise nicht mehr mittels mikrobiologischer Kulturverfahren 
nachweisbar sind [78, 79]. Die Abnahme geeigneter Probenmaterialien vor der Verabreichung von antimikrobiellen Substanzen führt $\mathrm{zu}$ einer Steigerung der Nachweisrate, sodass die Wahrscheinlichkeit der Identifizierung eines Pathogens höher ist. Die Isolierung eines oder mehrerer infizierender Mikroorganismen ermöglicht die Deeskalation der antimikrobiellen Therapie zunächst zum Zeitpunkt der Identifizierung. Eine weitere Anpassung der Therapie ist bei Vorliegen der Ergebnisse der Empfindlichkeitsprüfung möglich. Die Deeskalation der antimikrobiellen Therapie ist ein Kernbereich von ABS-Programmen und mit weniger Resistenzselektionsdruck, weniger Nebenwirkungen und geringeren Kosten verbunden [80]. Verschiedene retrospektive Studien deuten darauf hin, dass eine Abnahme der Probenmaterialien vor der antimikrobiellen Therapie mit einem verbesserten Outcome verbunden ist $[81,82]$. Auch die Deeskalation war in mehreren Beobachtungsstudien mit einer Verbesserung der Überlebensrate assoziiert [83, 84]. Das Bestreben, die Materialien für die mikrobiologische Diagnostik vor Beginn der antimikrobiellen Therapie zu entnehmen, muss gegen das Letalitätsrisiko bei einer Verzögerung des Beginns der kalkulierten antimikrobiellen Initialtherapie als wichtiger Therapiebaustein bei kritisch kranken Patienten mit dem Verdacht auf Sepsis oder septischen Schock abgewogen werden, bei denen ein signifikantes Sterberisiko besteht [85, 86].

Wir empfehlen, dass die Blutkulturen vor Beginn der antimikrobiellen Therapie abgenommen werden, sofern dies ohne wesentliche Zeitverzögerung möglich ist. Das Risiko-Nutzen-Verhältnis spricht jedoch für eine schnelle Verabreichung der antimikrobiellen Medikamente, falls es logistisch nicht möglich ist, die Probenmaterialien schnell abzunehmen. Daher sollten bei Patienten mit einem Verdacht auf Sepsis oder septischen Schock vor Beginn der antimikrobiellen Therapie geeignete Materialien für die mikrobiologische Diagnostik aus allen Lokalisationen abgenommen werden, die als potenzieller Fokus infrage kommen, soweit dadurch keine wesentliche Verzöge- rung in Bezug auf den Beginn der antimikrobiellen Therapie resultiert. In diesem Sinne geeignete Probenmaterialien sind z. B. Blutkulturen, Liquor, Urin, Wundund Atemwegsekrete und andere Körperflüssigkeiten. Probenmaterialien, deren Entnahme einen invasiven Eingriff erfordert wie z.B. eine Bronchoskopie oder einen offenen chirurgischen Eingriff, sind initial in der Regel wegen der damit verbundenen Zeitverzögerung bis zum Beginn der initialen antimikrobiellen Therapie nicht indiziert. Die Entscheidung, von welchen Lokalisationen Material abgenommen werden sollte, erfordert sorgfältige Überlegungen auf Seiten des Behandlungsteams. Von einer „Pan-Kultur“ aller möglichen Lokalisationen, von denen potenziell Proben entnommen werden können, wird abgeraten (es sei denn, der Sepsisherd ist klinisch nicht erkennbar), weil diese Praxis zu einem unangemessenen Einsatz von antimikrobiellen Substanzen führen kann [87]. Falls die Anamnese oder klinische Untersuchung eindeutig auf eine spezifische anatomische Lokalisation des Infektionsherds hindeutet, sind Materialien von anderen Lokalisationen (mit Ausnahme der Blutkulturen) im Allgemeinen nicht sinnvoll und nicht notwendig. Wir schlagen einen Zeitraum von 45 min für die Entnahme mikrobiologisch-diagnostischer Proben vor, der als keine wesentliche Verzögerung in Bezug auf den Beginn der antimikrobiellen Therapie betrachtet werden kann.

Zwei oder mehr Blutkultursets (bestehend aus mindestens aerober und anaerober Flasche) werden vor Beginn jeder neuen antimikrobiellen Therapie bei allen Patienten mit Verdacht auf Sepsis empfohlen [88]. Alle erforderlichen Blutkulturen können zum gleichen Zeitpunkt entnommen werden. Es gibt keinen Nachweis dafür, dass die Nachweisrate von Blutkulturen bei sequenziellen Entnahmen oder bei Abnahme im Temperaturanstieg höher ist $[89,90]$. Detaillierte Angaben zu geeigneten Entnahmeund Transportmethoden für Blutkulturproben sind in anderen Leitlinien enthalten $[90,91]$.

Bei potenziell septischen Patienten mit einem intravaskulären Katheter (der mehr als $48 \mathrm{~h}$ in situ ist), bei denen die Infektionslokalisation nicht klinisch erkennbar ist oder bei denen ein Verdacht auf eine intravaskuläre, katheterassoziierte Infektion besteht, sollte mindestens ein Blutkulturset aus dem Katheter entnommen werden (neben den gleichzeitig entnommenen peripheren Blutkulturen). Diese kann die Diagnose einer potenziell katheterassoziierten Blutstrominfektion stützen. Die Daten bezüglich des Nutzens der als Differential Time to Positivity (DTP) bekannten Untersuchungsmethode sind inkonsistent, was die Wahrscheinlichkeit betrifft, dass der vaskuläre Zugang den Infektionsherd darstellt [92-94]. Die Entnahme von Blutkulturen aus einem intravaskulären Katheter zur Diagnose einer katheterassoziierten Infektion soll nicht verhindern oder verzögern, dass der Katheter als potenzielle Infektionsquelle sofort nach der Probenahme entfernt wird (insbesondere bei nichtgetunnelten Kathetern).

Besteht beim Patienten kein Verdacht auf eine katheterassoziierte Infektion, wird jedoch ein anderer klinischer Infektionsherd vermutet, sollte mindestens eine Blutkultur (der 2 oder mehr Blutkulturen, die benötigt werden) peripher entnommen werden. Für die Entnahme der weiteren Blutkulturen kann keine Empfehlung gegeben werden, wo diese $\mathrm{zu}$ entnehmen sind. Die folgenden Optionen stehen zur Verfügung: a) Alle Blutkulturen werden peripher über eine Venenpunktion entnommen; b) die Kulturen werden über jeden einzelnen intravaskulären Zugang, aber nicht über mehrere Lumina ein und desselben intravaskulären Katheters entnommen oder c) die Kulturen werden über mehrere Lumina eines intravaskulären Zugangs entnommen [95-99].

In der nahen Zukunft könnten molekulardiagnostische Methoden die Möglichkeit bieten, Infektionen schneller und präziser zu diagnostizieren, als es mit den derzeit verfügbaren Methoden möglich ist. Obwohl unterschiedliche Technologien beschrieben worden sind, ist die klinische Erfahrung diesbezüglich bisher begrenzt und es werden zusätzliche Validierungen benötigt, bevor diese Methoden als Ergänzung oder Ersatz im Hinblick auf die Standardblut- 
kulturtechniken empfohlen werden können [100-102]. Außerdem werden die Empfindlichkeitsprüfungen der Mikroorganismen gegenüber den Antiinfektiva zumindest in der näheren Zukunft weiterhin erfordern, dass der Erreger angezüchtet und mittels phänotypischer Methoden direkt getestet wird.

\section{C.2 | Empfehlung | 2018

- Expertenkonsens
- Hyper- oder Hypothermie gehö-
ren zu den Kardinalsymptomen für
das Vorliegen einer Infektion. Wir
empfehlen für den Nachweis einer
Hyper- oder Hypothermie die Ver-
wendung von kalibrierten Methoden
entweder über zentrale Messungen
der Körperkerntemperatur (rektal,
zentralvenös, Harnblase, Ösophagus)
oder, falls nicht verfügbar, mittels
Infrarotthermometer in Ohr oder
Mund.
- Zusätzliche DSG-Leitlinienempfeh-
lung
- Konsensstärke: $100 \%$

Begründung. Kalibrierte Trommelfellthermometer oder orale Thermometer, die auf Infrarotmethoden beruhen, sind hinreichend genau. Periphere Thermometer haben allerdings keine klinisch akzeptable Genauigkeit zur Messung der Kerntemperatur im Vergleich zu zentralen Thermometern. In einer jüngsten Metaanalyse erfüllten nur kalibrierte Trommelfellthermometer oder Mundthermometer die Limits of Agreement (LOA), aber alle anderen Arten von peripheren Thermometern übertrafen die LOA im Vergleich zu zentralen Thermometern. Zum Nachweis von Fieber bei Erwachsenen und Kindern mit peripheren Thermometern betrug die gepoolte Sensitivität $64 \%$ (95\%-KI $55-72, \quad I^{2}=96 \%$ ), Spezifität $96 \%$ (KI 93-97, I ${ }^{2}=96 \%$ ). Die Likelihood-Ratio (LR) für einen positiven Test betrug $14,5\left(\mathrm{I}^{2}=94 \%\right)$ und das Wahrscheinlichkeitsverhältnis für einen negativen Test betrug $0,38\left(\mathrm{I}^{2}=97 \%\right)$ [103].

\section{Antimikrobielle Therapie}

\section{D.1 | Empfehlung | 2018}

- Wir empfehlen, dass die Verabreichung von intravenösen Antiinfektiva so schnell wie möglich, idealerweise innerhalb einer Stunde, nach der Diagnose einer Sepsis oder eines septischen Schocks erfolgt.

- SSC-Leitlinienadaptation

- Konsensstärke: $100 \%$

- Empfehlungsgrad: stark

- Evidenzgrad: moderat

Begründung. Bei Vorliegen einer Sepsis oder eines septischen Schocks ist jede Stunde Verzögerung in Bezug auf die Verabreichung geeigneter antimikrobieller Mittel mit einer messbaren Steigerung der Mortalität verbunden [86, 104]. Außerdem haben diverse Studien die negativen Auswirkungen von zunehmenden Verzögerungen auf die sekundären Endpunkte nachgewiesen (z.B. auf die Aufenthaltsdauer im Krankenhaus bzw. auf ITS [105], akute Nierenschädigungen [106], akute Lungenschädigungen [107] und Organschädigungen entsprechend der Beurteilung gemäß dem Sequential Organ Failure Assessment Score [108]). Trotz einer Metaanalyse von Studien meist niedriger Qualität, die keinen Nutzen einer schnellen antimikrobiellen Therapie nachgewiesen haben, unterstützen die größten Studien und die Studien mit der höchsten Qualität die möglichst schnelle Verabreichung von geeigneten antimikrobiellen Mitteln bei Patienten mit Sepsis mit oder ohne septischem Schock [86, 104, 109-111]. Die Mehrheit der Studien im Rahmen der Metaanalyse waren von niedriger Qualität, was auf eine Reihe von Mängeln zurückzuführen ist, einschließlich der geringen Fallzahlen, der Verwendung einer initialen Indexzeit mit einem willkürlichen Zeitpunkt wie der Ankunft in der Notaufnahme und der Indizierung des Ergebnisses in Bezug auf die zeitliche Verzögerung bis zum Ansetzen des ersten antimikrobiellen Mittels (ungeachtet der Wirksamkeit gegen das mutmaßliche Pathogen; [112, 113]).
Andere negative Studien, die nicht in diese Metaanalyse einbezogen wurden, sind durch das Gleichsetzen einer Bakteriämie mit einer Sepsis (die laut der aktuellen Definition ein Organversagen beinhalten muss) und einem septischen Schock von eingeschränkter Aussagekraft [114-117]. Viele dieser Studien werden auch durch die Indizierung der Verzögerung in Bezug auf leicht verfügbare, aber nichtphysiologische Variablen kompromittiert wie z. B. dem Zeitpunkt der initialen Probenahme von Blutkulturen (ein Ereignis, das wahrscheinlich eine hohe Variabilität aufweist, was den zeitlichen Faktor betrifft).

Während die verfügbaren Daten nahelegen, dass die frühestmögliche intravenöse Verabreichung geeigneter antimikrobieller Mittel nach der Identifizierung einer Sepsis oder eines septischen Schocks zu optimalen Ergebnissen führt, wird eine Stunde als angemessenes Mindestziel empfohlen. Die Umsetzbarkeit bezüglich des konsistenten Erreichens dieses Ziels ist jedoch noch nicht ausreichend analysiert worden. Praktische Betrachtungen, wie z.B. die Herausforderungen in Bezug auf die frühzeitige Identifizierung der Patienten durch die Kliniker oder operative Probleme in der Verfügbarkeit von Antiinfektiva, stellen wenig untersuchte Variablen dar, die das Erreichen dieses Ziels beeinträchtigen könnten. Eine Reihe von patienten- und organisationsbezogenen Faktoren scheinen die Verzögerungen der Verabreichung von geeigneten antimikrobiellen Mitteln zu beeinflussen [118].

Eine Optimierung der Compliance mit dieser Empfehlung setzt eine Analyse der Ursachen von Verzögerungen auf institutioneller Seite voraus [119]. Eine inakzeptabel hohe Häufigkeit des Nichterkennens des potenziellen Vorliegens einer Sepsis oder eines septischen Schocks oder das Ansetzen einer nichtgeeigneten empirischen antimikrobiellen Therapie (z. B. infolge einer Nichtbeachtung des Potenzials einer mikrobiellen Resistenz oder der kürzlichen Verwendung eines Antiinfektivums) können als Hinweise auf Defizite gewertet werden.

Die Einrichtung eines vaskulären $\mathrm{Zu}$ gangs und das Ansetzen einer aggressiven Flüssigkeitstherapie sind von hoher 
Bedeutung bei der Behandlung von Patienten mit Sepsis oder septischem Schock. Eine unverzügliche intravenöse Infusion mit antimikrobiellen Mitteln hat ebenfalls höchste Priorität. Dazu könnten zusätzliche vaskuläre Zugänge erforderlich sein. Ein intraossärer Zugang, der schnell und verlässlich eingerichtet werden kann (auch bei Erwachsenen), kann verwendet werden, um die Initialdosen jedweder antimikrobieller Mittel zu verabreichen [120, 121]. Außerdem sind einige intramuskuläre Präparate zugelassen und verfügbar, was diverse $\beta$-Laktame zur Erstlinientherapie betrifft, zu denen Imipenem/Cilastatin, Cefepim, Ceftriaxon und Ertapenem gehören. Einige weitere $\beta$-Laktame zur Erstlinientherapie können in Notfallsituationen ebenfalls wirksam intramuskulär verabreicht werden, falls kein vaskulärer oder intraossärer $\mathrm{Zu}$ gang zur Verfügung steht, obwohl eine regulatorische Zulassung der intramuskulären Verabreichung dieser Medikamente nicht vorliegt [122-124]. Die intramuskuläre Absorption und Verteilung einiger dieser Wirkstoffe bei schweren Erkrankungen ist noch nicht erforscht, daher sollte die intramuskuläre Verabreichung nur in Betracht gezogen werden, wenn eine zeitgerechte Einrichtung eines vaskulären Zugangs nicht möglich ist.

\section{D.2 | Empfehlung | 2018}

- Wir empfehlen eine empirische Breitspektrumtherapie mit einem

Antibiotikum oder mehreren Antibiotika bei Patienten mit Sepsis oder septischem Schock, um alle wesentlichen Bakterien zu erfassen.

- SSC-Leitlinienadaptation

- Konsensstärke: $100 \%$

- Empfehlungsgrad: stark

- Evidenzgrad: moderat

\section{D.3 | Empfehlung | 2018}

\section{- Expertenkonsens}

- Wir empfehlen, die Indikation einer zusätzlichen kalkulierten antimykotischen oder antiviralen Therapie bei Risikopatienten entsprechend der fokusbezogenen Leitlinie zu überprüfen.

\section{- Zusätzliche DSG-Leitlinienempfeh- lung \\ - Konsensstärke: $100 \%$}

\section{D.4 | Empfehlung | 2018}

- Expertenkonsens
- Wir empfehlen, dass die kalkulierte
antiinfektive Initialtherapie ange-
passt wird, sobald ein plausibler Erre-
gernachweis geführt werden konnte
und die Antiinfektivaempfindlichkeit
bekannt ist. Wir empfehlen, dass bei
klinischer Verbesserung des Patien-
ten innerhalb der ersten $72 \mathrm{~h}$ auch
ohne Erregernachweis eine initia-
le Kombinationstherapie auf eine
Monotherapie deeskaliert wird.
SSC-Leitlinienmodifikation
Konsensstärke: $100 \%$

Begründung. Die Initiierung einer geeigneten antimikrobiellen Therapie (d.h. einer wirksamen Therapie gegen das bzw. die verursachende[n] Pathogen[e]) ist einer der wichtigsten Aspekte des effektiven Managements von lebensbedrohlichen Infektionen, die eine Sepsis und einen septischen Schock verursachen. Das Unterlassen einer geeigneten empirischen Therapie bei Patienten mit Sepsis und septischem Schock ist mit einer beträchtlichen Zunahme der Morbidität und Mortalität verbunden [109, 125-127]. Außerdem ist die Wahrscheinlichkeit einer Progression von einer gramnegativen bakteriämischen Infektion zu einem septischen Schock erhöht [128]. Dementsprechend muss die initiale Auswahl einer antimikrobiellen Therapie breit genug angelegt sein, um alle wahrscheinlichen Pathogene abzudecken. Die Auswahl der empirischen antimikrobiellen Therapie hängt von komplexen Fragen im Zusammenhang mit der medizinischen Vorgeschichte des Patienten, dem klinischen $\mathrm{Zu}$ stand und lokalen epidemiologischen Faktoren ab. Zu den wichtigsten patientenbezogenen Faktoren gehören die Art des klinischen Syndroms/die Lokalisation der Infektion, die zugrunde liegenden Begleiterkrankungen, chronisches Organversagen, Medikamente,
Dauerkatheter, das Vorliegen einer Immunsuppression oder einer anderen Beeinträchtigung des Immunsystems, eine kürzlich bekanntgewordene Infektion oder Kolonisierung mit spezifischen Pathogenen sowie eine Behandlung mit Antiinfektiva innerhalb der letzten $3 \mathrm{Mo-}$ nate. Außerdem müssen Faktoren wie der Aufenthaltsort des Patienten zum Zeitpunkt der Infektion (z. B. kommunaler Bereich, Versorgungseinrichtung für chronisch Kranke, Akutversorgungshospital), die lokale Pathogenprävalenz und die Suszeptibilitätsmuster dieser Pathogene sowohl auf kommunaler Ebene als auch im Krankenhaus in der Therapiewahl berücksichtigt werden. Auch Medikamentenunverträglichkeiten und Toxizitäten müssen berücksichtigt werden.

Die häufigsten Pathogene, die einen septischen Schock verursachen, sind gramnegative Bakterien sowie grampositive Mikroorganismen. Bei ausgewählten Patienten sollten eine invasive Candidiasis, ein toxisches Schocksyndrom und auch selten auftretende Pathogene in Betracht gezogen werden. Bestimmte Sondersituationen setzen Patienten einem Risiko für atypische oder resistente Pathogene aus. Beispielsweise sind Neutropeniepatienten einem Risiko in Bezug auf ein besonders breites Spektrum an potenziellen Pathogenen ausgesetzt, zu denen resistente gramnegative Bakterien und Kandidaspezies gehören. Patienten mit nosokomial erworbenen Infektionen sind anfällig für eine Sepsis mit dem methicillinresistenten Staphylococcus aureus (MRSA) und mit vancomycinresistenten Enterokokken (VRE).

Die Auswahl einer optimalen empirischen antimikrobiellen Behandlung bei Sepsis und septischem Schock ist einer der zentralen Einflussfaktoren in Bezug auf das Outcome. Die Überlebensrate kann sich um das 5-Fache verringern, wenn ein septischer Schock mit einer empirischen Therapie behandelt wird, die das ursächliche Pathogen nicht abdeckt [125]. Wegen der hohen Mortalität, die mit einer nichtadäquaten initialen Therapieverbunden ist, sollten empirische Therapien im Zweifelsfall ein breites Spektrum von Pathogenen berücksichtigen. Die Auswahlvon empirischen antimikro- 
biellen Therapien bei Patienten mit Sepsis und septischem Schock ist jedoch eine komplexe Aufgabe und kann nicht auf eine einfache Tabelle reduziert werden. Diverse Faktoren müssen berücksichtigt werden, um eine geeignete antimikrobielle Therapie zu finden, und zwar an jedem medizinischen Zentrum und für jeden einzelnen Patienten. Dazu gehören:

a) die anatomische Lokalisation des

Infektionsherds unter Beachtung des typischen Pathogenprofils und der

Gewebegängigkeit der Antiinfektiva;

b) prävalente Pathogene auf kommunaler Ebene, im Krankenhaus und auf der jeweiligen Station des Krankenhauses;

c) die Resistenzmuster dieser prävalenten Pathogene;

d) das Vorliegen von spezifischen Immundefekten, wie z. B. Neutropenie, Z.n. Splenektomie, eine schlecht kontrollierte HIV-Infektion, erworbene oder angeborene Immunglobulindefekte bzw. Störungen der Leukozytenfunktion;

e) das Alter und die Komorbiditäten des betreffenden Patienten einschließlich chronischer Erkrankungen (z. B. Diabetes) und chronischer Organdysfunktionen (z. B. Leber- oder Nierenversagen), das Vorliegen von Devices (z. B. zentralvenöse Zugänge oder Urinkatheter), die die Abwehr gegenüber Infektionen kompromittieren.

Angesichts der Bandbreite der Variablen, die analysiert werden müssen, ist die Empfehlung einer spezifischen Behandlung bei einer Sepsis und einem septischen Schock nicht möglich. Die Leser dieses Dokuments werden auf Leitlinien verwiesen, die Informationen $\mathrm{zu}$ Behandlungsmöglichkeiten basierend auf der anatomischen Lokalisation der Infektion oder spezifischen Immundefekten zur Verfügung stellen [96, 129-139].

Eine Kombinationstherapie ist häufig erforderlich, um zu Beginn ein ausreichend breites Spektrum der empirischen Abdeckung sicherzustellen. Kliniker sollten sich des Risikos einer Resistenz gegenüber Breitspektrum- $\beta$-Laktamen und Carbapenemen in Bezug auf gramnegative Bazillen in einigen kommunalen Bereichen und Einrichtungen des Gesundheitswesens bewusst sein. Die Ergänzung eines zusätzlichen gramnegativen Wirkstoffs zu der empirischen Behandlung wird bei kritisch erkrankten septischen Patienten empfohlen, die einem hohen Risiko einer Infektion mit derartigen multiresistenten Pathogenen ausgesetzt sind (z. B. Pseudomonas, Acinetobacter etc.). So soll die Wahrscheinlichkeit erhöht werden, dass zumindest ein aktiver Wirkstoff verabreicht wird [140]. Ebenso ist in Situationen mit einem Risiko für andere resistente oder atypische Pathogene die Ergänzung eines pathogenspezifischen Wirkstoffs zur Erweiterung des Spektrums gerechtfertigt. Vancomycin, Teicoplanin oder ein anderer Anti-MRSA-Wirkstoff können genutzt werden, wenn Risikofaktoren für MRSA vorhanden sind. Ein signifikantes Risiko einer Infektion mit LegionellaSpezies macht eine Kombinationstherapie mit einem Makrolid oder Fluorchinolon erforderlich.

Auch eine Kandidainfektion sollte in Erwägung gezogen werden. $\mathrm{Zu}$ den Risikofaktoren für invasive Kandidainfektionen gehören eine Beeinträchtigung des Immunsystems (Neutropenie, Chemotherapie, Transplantation, Diabetes mellitus, chronisches Leberversagen, chronisches Nierenversagen), eine längere Nutzung von invasiven vaskulären Zugängen (Hämodialysekatheter, zentralvenöse Katheter), eine vollumfängliche parenterale Ernährung, eine nekrotisierende Pankreatitis, ein kurz zuvor erfolgter großer chirurgischer Eingriff (insbesondere im Abdominalbereich), eine längere Verabreichung von Breitspektrumantibiotika, ein längerer Krankenhausaufenthalt, eine kurz zurückliegende Pilzinfektion und eine Kolonisierung an mehreren Lokalisationen [141, 142]. Falls ein Risiko für eine Kandidasepsis besteht und eine empirische antimykotische Behandlung gerechtfertigt erscheint, sollte die Auswahl des spezifischen Wirkstoffs basierend auf dem Schweregrad der Erkrankung, dem lokalen Muster der prävalentesten Kandidaspezies und einer etwaigen kürzlichen Exposition gegenüber antimykotischen Medikamenten erfolgen.
Die empirische Nutzung von Echinocandinen (Anidulafungin, Micafungin oder Caspofungin) wird bei den meisten Patienten mit schwerer Erkrankung vorgezogen, insbesondere bei denjenigen Patienten mit septischem Schock, die vor Kurzem mit anderen antimykotischen Wirkstoffen behandelt wurden, oder falls eine Infektion mit Candida glabrata oder Candida krusei aufgrund von früheren Kulturdaten vermutet wird [130, 135]. Triazole sind bei hämodynamisch stabilen, weniger schwer erkrankten Patienten akzeptabel, bei denen keine vorherige Triazolexposition vorliegt und die nicht bekanntermaßen mit azolresistenten Spezies kolonisiert sind. Liposomale Formulierungen von Amphotericin B sind eine angemessene Alternative $\mathrm{zu}$ Echinocandinen bei Patienten mit Echinocandinintoleranz oder -toxizität [130, 135]. Erkenntnisse über die lokalen Resistenzmuster gegenüber antimykotischen Wirkstoffen sollten die Grundlage für die Medikamentenauswahl bilden, bis mykotische Suszeptibilitätsresultate vorliegen (falls verfügbar). Schnelle diagnostische Analysen unter Verwendung von $\beta$-D-Glucan- oder Polymerasekettenreaktionsassays können hilfreich in der Therapieentscheidung sein. Der negative prädiktive Wert derartiger Tests ist jedoch nicht ausreichend hoch, um eine primäre Therapieentscheidung davon abhängig zu machen.

Eine überlegene empirische Abdeckung kann durch die Nutzung von lokalen und stationsspezifischen Resistenzmustern [143, 144] oder die Konsultation von Infektiologen erreicht werden [145]. Eine frühzeitige Einbeziehung von Infektiologen kann unter gewissen Umständen (z. B. bei einer Staphylococcusaureus-Bakteriämie) das Behandlungsergebnis verbessern [143-145].

Bei Patienten mit Sepsis oder septischem Schock ist im Allgemeinen eine empirische Breitspektrumtherapie solange gerechtfertigt, bis der verursachende Organismus und dessen antimikrobielle Suszeptibilität bekannt sind. An diesem Punkt sollte das Therapiespektrum eingegrenzt werden, indem nichterforderliche antimikrobielle Wirkstoffe abgesetzt und Breitspektrumwirkstoffe durch spezifischere Wirkstoffe ersetzt werden 
[146]. Falls die relevanten Kulturen negativ sein sollten, ist eine empirische Therapiebeschränkung basierend auf einem guten klinischen Ansprechen angemessen. Die Nutzung von ABS-Programmen wird in diesem Zusammenhang befürwortet.

Bei etwa einem Drittel der Patienten mit einer Sepsis kann kein verursachendes Pathogen identifiziert [125] werden. Das ist überwiegend dann der Fall, wenn die Kulturen unter bereits laufender antimikrobieller Therapie abgenommen wurden. In einigen Fällen könnte dies darauf zurückzuführen sein, dass in einer Studie post hoc festgestellt wurde, dass fast die Hälfte der Patienten mit initialem Verdacht auf Sepsis gar keine Infektion hatten bzw. eine Infektion nur „möglich“ (aber nicht wahrscheinlich) erschien. Falls hinreichend nachgewiesen werden kann, dass keine Infektion vorliegt, sollte die antimikrobielle Therapie daher unverzüglich beendet werden.

\section{D.5 | Empfehlung | 2018}

\section{- Expertenkonsens \\ - Wir empfehlen, dass von einer an- haltenden systemischen antimikro- biellen Prophylaxe bei Patienten mit schweren inflammatorischen Zu- ständen nichtinfektiösen Ursprungs abgesehen wird (z. B. schwere Pan- kreatitis, Brandverletzung). \\ - SSC-Leitlinienadaptation \\ - Konsensstärke: $100 \%$}

Begründung. Eine systemische entzündliche Reaktion ohne Infektion rechtfertigt keine antimikrobielle Therapie. Dazu gehören z. B. eine schwere Pankreatitis und großflächige Brandwunden.

Obwohl die prophylaktische Verwendung systemischer Antibiotika bei einer schweren nekrotisierenden Pankreatitis in der Vergangenheit empfohlen wurde, sprechen sich aktuelle Leitlinien dagegen aus [147]. Dieses wird durch eine aktuelle Metaanalyse bestätigt, die keinen klinischen Vorteil von prophylaktisch verabreichten Antibiotika nachwies, der deren langfristige Nebenwirkungen aufwiegen würde [148]. In gleicher Weise wurde eine längere systemische antimi- krobielle Prophylaxe für Patienten mit schweren Verbrennungen durchgeführt. Aktuelle Metaanalysen weisen jedoch auf den fraglichen klinischen Nutzen dieses Ansatzes hin [149, 150]. Die aktuellen Leitlinien für die Behandlung von Brandwunden unterstützen die Aufrechterhaltung einer antimikrobiellen Prophylaxe nicht [132]. Die vorhandende Evidenz ist jedoch gering, was auf die Diversität der Population zurückzuführen ist. Die Evidenzqualität war niedrig für die Mortalität bei Pankreatitis [148] und ebenfalls niedrig bei Verbrennungen; daher glauben wir, dass diese Empfehlung besser als Expertenkonsens zu deklarieren ist, der besagt, dass die Alternative der Verabreichung von Antibiotika ohne Anzeichen einer Infektion nicht plausibel ist [148-150]. Trotz unserer Empfehlung, von einer längeren systemischen antimikrobiellen Prophylaxe im Allgemeinen abzusehen, könnte eine kurze antibiotische Prophylaxe für spezifische invasive Eingriffe angemessen sein. Außerdem gilt, dass ein starker Verdacht bezüglich des gleichzeitigen Vorliegens einer Sepsis oder eines septischen Schocks bei Patienten mit einem schweren inflammatorischen $\mathrm{Zu}$ stand nichtinfektiösen Ursprungs eine antimikrobielle Therapie rechtfertigt.

\section{D.6 | Empfehlung | 2018}

\section{- Expertenkonsens wird. \\ - SSC-Leitlinienadaptation \\ - Konsensstärke: $100 \%$}

- Wir empfehlen, dass die Dosierung der Antiinfektiva basierend auf anerkannten pharmakokinetischen/ pharmakodynamischen Prinzipien und spezifischen Medikamenteneigenschaften bei Patienten mit Sepsis oder septischem Schock optimiert

Begründung. Eine frühzeitige Optimierung der antimikrobiellen Pharmakokinetik kann das Outcome von Patienten mit einer schweren Infektion verbessern. Verschiedene Aspekte sollten bei der Dosisfindung berücksichtigt werden. Bei Patienten mit Sepsis oder septischem Schock liegt häufig eine hepatische oder renale Dysfunktion vor. Weiterhin bestehen immunologische Dysfunktionen und eine erhöhte Prädisposition in Bezug auf Infektionen mit resistenten Organismen. Wahrscheinlich am wichtigsten in Bezug auf die initiale antimikrobielle Dosierung ist ein erhöhtes Distributionsvolumen für die meisten Antibiotika, was teilweise auf die schnelle Expansion des extrazellulären Volumens als Folge einer aggressiven Flüssigkeitstherapie zurückzuführen ist. Dies führt zu einer unerwartet hohen Häufigkeit von suboptimalen Medikamentenkonzentrationen bei einem großen Spektrum von Antibiotika.

Antibiotika haben unterschiedliche Plasmazielwerte, die anzustreben sind. Das Nichterreichen von maximalen Plasmazielwerten bei der Inititaldosis wurde mit einem Therapieversagen von Aminoglykosiden nachgewiesen [151]. Ebenso wird ein unzureichender früher Talspiegel von Vancomycin in Bezug auf den Spitzenplasmaspiegel (im Verhältnis zur minimalen Hemmstoffkonzentration $[\mathrm{MHK}]) /$ „,minimal inhibition concentration“ [MIC]) mit einem klinischen Versagen bei schweren MRSA-Infektionen in Zusammenhang gebracht ([152]; einschließlich nosokomialer Pneumonie [153] und septischem Schock [154]). Die klinische Erfolgsrate bei der Behandlung schwerer Infektionen korreliert mit einem höheren Spitzenwert für die Blutkonzentration (im Verhältnis zur Pathogen-MIC) der Fluorchinolone (nosokomiale Pneumonie und andere schwere Infektionen; [155-157]) und Aminoglykoside (gramnegative Bakteriämie, nosokomiale Pneumonie und andere schwere Infektionen; [158]). Bei $\beta$-Laktamen scheinen überlegene klinische und mikrobiologische Erfolgsraten mit einer längeren Dauer in Bezug auf höhere Plasmaspiegel im Verhältnis zur Pathogen-MIC verbunden zu sein, was insbesondere kritisch erkrankte Patienten betrifft [159-162].

Die optimale Dosierungsstrategie für Aminoglykoside und Fluorchinolone beinhaltet eine Optimierung der Plasmaspitzenwerte. Bei Aminoglykosiden kann dies am einfachsten durch eine einmalige tägliche Dosisgabe $(5-7 \mathrm{mg} / \mathrm{kg}$ tägliche Gentamicinäquivalentdosis) erreicht 
werden. Die einmal tägliche Dosisgabe führt zumindest $\mathrm{zu}$ einer vergleichbaren klinischen Wirksamkeit bei möglicherweise reduzierter renaler Toxizität im Vergleich zur mehrmals täglichen Dosisgabe $[163,164]$. Die einmal tägliche Dosisgabe von Aminoglykosiden wird bei Patienten mit erhaltener Nierenfunktion genutzt. Patienten mit einer leichten chronischen Beeinträchtigung der Nierenfunktion sollten dennoch eine einmal tägliche Äquivalentdosis erhalten, wobei bei diesen Patienten üblicherweise ein größerer Zeitraum (von bis zu 3 Tagen) ohne Dosisgabe vor der nächsten Dosis eingehalten wird. Dieses Dosierungsschema sollte nicht bei Patienten mit schwerer Beeinträchtigung der Nierenfunktion genutzt werden, bei denen keine Ausscheidung der Aminoglykoside innerhalb von wenigen Tagen zu erwarten ist. Das therapeutische Drugmonitoring der Aminoglykoside in diesem Kontext soll hauptsächlich sicherstellen, dass die Talspiegel ausreichend gering sind, um das Potenzial für eine renale Toxizität $\mathrm{zu}$ minimieren. In Bezug auf die Fluorchinolone ist ein Ansatz, der die Dosis innerhalb eines nichttoxischen Bereichs optimiert (z. B. Ciprofloxacin, $600 \mathrm{mg}$ alle $12 \mathrm{~h}$, oder Levofloxacin, $750 \mathrm{mg}$ alle $24 \mathrm{~h}$, ausgehend von einer erhaltenen Nierenfunktion), mit einem verbesserten mikrobiologischen und klinischen Therapieerfolg verbunden [165-167].

Vancomycin ist ein weiteres Antibiotikum, dessen Wirksamkeit zumindest teilweise konzentrationsabhängig ist. Die Dosierung mit einem Talspiegel von $15-20 \mathrm{mg} / \mathrm{l}$ wird von Experten empfohlen, um die Wahrscheinlichkeit des Erreichens angemessener pharmakodynamischer Ziele zu maximieren, die Gewebepenetration zu verbessern und die klinischen Ergebnisse zu optimieren [168-170]. Ein Monitoring der Talspiegel vor der nächsten Dosisgabe wird empfohlen. Bei Sepsis und septischem Schock wird eine intravenöse Sättigungsdosis von $25-30 \mathrm{mg} / \mathrm{kg}$ (basierend auf dem tatsächlichen Körpergewicht) vorgeschlagen, um schnell die avisierten Talspiegel zu erreichen. Eine Aufsättigungsdosis („loading dose“) von $1 \mathrm{~g}$ Vancomycin wird die frühzeitigen therapeutischen Konzentrationen bei einer signifikanten Untergruppe der Patienten nicht erreichen können. Tatsächlich sind Aufsättigungsdosen von Antibiotika mit niedrigem Distributionsvolumen (Teicoplanin, Vancomycin, Colistin) bei kritisch kranken Patienten gerechtfertigt, um schneller therapeutische Medikamentenkonzentrationen $\mathrm{zu}$ erreichen, was auf deren erweitertes extrazelluläres Volumen im Zusammenhang mit der Volumenexpansion nach der Flüssigkeitstherapie zurückzuführen ist [171-175]. Aufsättigungsdosen werden auch für $\beta$-Laktame empfohlen, die als kontinuierliche oder langsam („extended“) verabreichte Infusionen verabreicht werden, um die Akkumulation des Medikaments in Bezug auf therapeutische Konzentrationen zu beschleunigen [176]. $\mathrm{Zu}$ beachten ist, dass die erforderliche Aufsättigungsdosis von antimikrobiellen Medikamenten nicht durch Änderungen der Nierenfunktion beeinträchtigt wird, obwohl dadurch die Verabreichungshäufigkeit und/oder die tägliche Gesamtdosis beeinflusst werden könnten.

Bei $\beta$-Laktamen korreliert der wichtigste pharmakodynamische Parameter mit der mikrobiologischen und klinischen Reaktion. Dabei handelt es sich um die Zeit, in der die Plasmakonzentration des Medikaments die PathogenMIC im Verhältnis zum Dosisintervall übersteigt (T > MIC). Ein Mindestunterschied in Bezug auf $\mathrm{T}>\mathrm{MIC}$ in Höhe von $60 \%$ ist im Allgemeinen ausreichend, um ein gutes klinisches Ansprechen bei einer leichten bis mittelschweren Erkrankung zu ermöglichen. Ein optimales Ansprechen bei schweren Infektionen einschließlich Sepsis kann jedoch mit einem Unterschied in Bezug auf T > MIC in Höhe von $100 \%$ erreicht werden [161]. Der einfachste Weg, um einen höheren Unterschied in Bezug auf T $>$ MIC zu erreichen, besteht in einer erhöhten Dosisfrequenz (bei einer identischen täglichen Dosis). Beispielsweise kann Piperacillin/Tazobactam entweder in Höhe von $4,5 \mathrm{~g}$ alle $8 \mathrm{~h}$ oder $3,375 \mathrm{~g}$ alle $6 \mathrm{~h}$ bei schweren Infektionen dosiert werden. Wenn alle sonstigen Umstände übereinstimmen, würde durch die letztgenannte Dosis ein höherer Unterschied in Bezug auf $\mathrm{T}>$ MIC erreicht werden.
Wir haben zuvor vorgeschlagen, dass initiale Dosen an $\beta$-Laktamen als Bolus oder Kurzinfusion verabreicht werden, um schnell eine therapeutische Blutkonzentration zu erreichen. Nach der initialen Dosis sollte jedoch bevorzugt eine verlängerte Infusion des Medikaments über mehrere Stunden (zur Erhöhung des Unterschieds in Bezug auf $\mathrm{T}>\mathrm{MIC}$ ) verabreicht werden, anstelle der dem Standard entsprechenden 30-minütigen Infusion, die von einigen Autoren empfohlen wird [177, 178]. Außerdem deuten manche Metaanalysen darauf hin, dass eine verlängerte/ kontinuierliche Infusion von $\beta$-Laktamen effektiver sein könnte als eine intermittierende Kurzinfusion, insbesondere bei relativ resistenten Organismen und Patienten mit Sepsis [162, 179-181]. Eine aktuelle individuelle Patientendatenmetaanalyse von randomisierten kontrollierten Studien zum Vergleich von kontinuierlichen und intermittierenden Infusionen von $\beta$-Laktam-Antibiotika bei Patienten mit schwerer Sepsis ergab einen unabhängigen schützenden Effekt einer kontinuierlichen Therapie nach Adjustierung um andere korrelierte Ergebnisdaten [162].

Während die vorliegende Evidenz pharmakokinetisch optimierte antimikrobielle Dosisstrategien bei Patienten mit Sepsis und septischem Schock unterstützt, ist dies in der Praxis schwer umsetzbar, ohne dass schnelle Verfahren für ein therapeutisches Drugmonitoring verfügbar sind (d.h. Vancomycin, Teicoplanin und Aminoglykoside). Septische Patienten zeigen eine Vielfalt von physiologischen Störungen auf, die die Pharmakokinetik von Antiinfektiva beeinflussen. Dazu gehören eine instabile Hämodynamik, ein erhöhtes Herzzeitvolumen, ein erhöhtes extrazelluläres Volumen, eine variable Nierenund Leberperfusion (die die Medikamentenclearance beeinträchtigen) und eine geänderte Medikamentenbindung aufgrund des reduzierten Serumalbumins [162]. Darüber hinaus könnte eine erhöhte renale Clearance zu einer verringerten antimikrobiellen Serumkonzentrationen in der frühen Phase der Sepsis führen. Basierend auf Studien mit therapeutischem Drugmonitoring 
ist eine Unterdosierung (insbesondere in der frühen Behandlungsphase) bei septischen Patienten häufig, wobei jedoch auch Medikamententoxizitäten, wie z.B. eine Irritation des zentralen Nervensystems beim Einsatz von $\beta$-Laktamen und Nierenschäden bei der Verwendung von Colistin, zu berücksichtigen sind [182-185]. Dieses unterstreicht die Bedeutung eines erweiterten therapeutischen Drugmonitorings bei Patienten mit Sepsis und septischem Schock.

\section{D.7 | Empfehlung | 2018}

\section{- Wir schlagen vor, dass eine kalku- lierte Kombinationstherapie nicht routinemäßig zur Behandlung der Sepsis eingesetzt wird. \\ - Bemerkungen: Davon ausgenommen sind Situationen, bei denen für die auslösende Infektion in den entsprechenden Leitlinien eine Kombinationstherapie empfohlen wird (z. B. Endokarditis, ambulant erworbene Pneumonie, Toxic-shock- Syndrom, ZNS-Infektionen, hohes Risiko für multiresistente Erreger). \\ - SSC-Leitlinienadaptation und SSC- Leitlinienmodifikation \\ - Konsensstärke: $100 \%$ \\ - Empfehlungsgrad: schwach \\ - Evidenzgrad: niedrig}

\section{D.8 | Empfehlung | 2018}

- Wir empfehlen, dass von einer Kombinationstherapie zur routinemäßigen Behandlung von neutropenischer Sepsis/Bakteriämie abgesehen wird.

- SSC-Leitlinienadaptation

- Konsensstärke: $100 \%$

- Empfehlungsgrad: stark

- Evidenzgrad: moderat

Begründung. Die Bezeichnung „Kombinationstherapie“ im Kontext dieser Leitlinie bezieht sich auf die Verwendung von 2 verschiedenen Antibiotikaklassen (üblicherweise ein $\beta$-Laktam mit einem Fluorchinolon, Aminoglykosid oder Makrolid) für ein einzelnes Pathogen, von dem erwartet wird, dass dieses eine hohe Empfindlichkeit auf beide Wirkstoffe aufweist und dass durch die Kombination die Clearance des Pathogens beschleunigt wird. Der Begriff wird nicht in Fällen verwendet, in denen der Zweck einer Kombinationstherapie ausdrücklich darin besteht, das Spektrum der antimikrobiellen Aktivität zu erweitern (z. B. Vancomycin in Ergänzung zu Ceftazidim, Metronidazol in Ergänzung zu einem Aminoglykosid oder ein Echinocandin in Ergänzung zu einem $\beta$-Laktam).

Eine Studie hat mittels Propensitymatching-Verfahren bzw. Metaanalyse/ Metaregressionsanalyse Hinweise darauf gegeben, dass eine Kombinationstherapie mit einer höheren Überlebensrate bei schwer erkrankten Patienten mit einem hohen Sterberisiko assoziiert ist, insbesondere bei Patienten mit septischem Schock [186, 187]. Die Metaregressionsstudie [186] wies auf den Nutzen einer Kombinationstherapie bei Patienten mit einem Mortalitätsrisiko von mehr als $25 \%$ hin. Ebenso haben diverse Beobachtungsstudien einen Überlebensvorteil mit hohem Sterberisiko aufgezeigt [188-191]. Die zuvor erwähnte Metaregressionsanalyse hat jedoch auch auf die Möglichkeit eines erhöhten Sterberisikos bei Verwendung einer Kombinationstherapie bei Niedrigrisikopatienten (Mortalitätsrisiko $<15 \%)$ ohne septischen Schock hingewiesen [186]. Eine kontrollierte Studie zeigte, dass bei der Verwendung eines Carbapenems als empirische Therapie bei einer Population mit einem niedrigen Infektionsrisiko in Bezug auf resistente Mikroorganismen die Kombination mit einem Fluorchinolon nicht $\mathrm{zu}$ einer Verbesserung des Outcomes führt [192]. Trotz der insgesamt vorteilhaften Evidenz für eine Kombinationstherapie bei septischem Schock steht keine direkte Evidenz aus RCT mit angemessener Aussagekraft zur Verfügung. Möglicherweise ist bei septischem Schock eine vorübergehende Kombinationstherapie nützlich [175, 186, 187]. Dagegen gibt es auch keine Hinweise für ein besseres klinisches Outcome bei Patienten mit Bakteriämie und Sepsis ohne Schock.

Eine Reihe von weiteren Beobachtungsstudien und einige kleine prospektive Studien unterstützen eine initiale Kombinationstherapie für ausgewählte
Patienten mit spezifischen Pathogenen (z.B. schwere Pneumokokkeninfektion, multiresistente gramnegative Pathogene; [191, 193-199]). Leider ist das verursachende Pathogen in den meisten Fällen nicht bekannt. Daher ist eine Kombinationstherapie in Bezug auf spezifische identifizierte Pathogene nur sinnvoll, wenn eine längere, gezielte Kombinationstherapie in Betracht gezogen wird. Bei Vorliegen von multiresistenten $\mathrm{Pa}$ thogenen hat eine Kombinationstherapie keine eindeutigen Vorteile. Außerdem führen sowohl individuelle Studien als auch Metaanalysen im Hinblick auf multiresistente Pathogene $\mathrm{zu}$ variablen Ergebnissen in Abhängigkeit vom Pathogen und dem klinischen Szenario [196, 198-201]. Eine Konsultation durch einen Infektiologen kann ratsam sein, wenn ein Verdacht auf multiresistente Pathogene besteht. Breiter Konsens in Bezug auf die Nutzung einer spezifischen Form einer Kombinationstherapie besteht beim toxischen Streptokokkenschocksyndrom [139, 202, 203].

Obwohl die Datenlage den Nutzen einer initialen Kombinationstherapie bei septischem Schock unterstützt, wurde die Wirksamkeit einer weiterführenden anhaltenden Behandlung schwerer Infektionen (einschließlich Bakteriämie und Sepsis ohne septischen Schock) bisher nicht nachgewiesen [204]. Der Begriff "anhaltende Behandlung" beinhaltet eine erweiterte empirische Therapie bei kulturnegativen Infektionen und eine erweiterte definitive/gezielte Therapie in Fällen, in denen ein Pathogen identifiziert wurde. Im Fall einer Neutropenie unter Abwesenheit eines septischen Schocks deuten Studien unter Verwendung moderner Breitspektrumantibiotika durchgängig darauf hin, dass eine Kombinationstherapie zur Erweiterung der Pathogenabdeckung (z. B. unter Einbeziehung von Kandidaspezies) zwar hilfreich sein kann, eine Kombinationstherapie unter Verwendung eines $\beta$-Laktams und eines Aminoglykosids aber nicht vorteilhaft für weniger schwer erkrankte „Niedrigrisikopatienten“ ist [205]. Eine Kombinationstherapie dieser Art wird sogar für „Hochrisikopatienten“ mit Neutropenie (einschließlich einer hämodynamischen Instabilität und Organversagen), bei de- 
nen eine Sepsis vorliegt, von verschiedenen internationalen Expertengruppen nicht durchgängig unterstützt [136].

Daten hoher Qualität bezüglich der klinisch gesteuerten Deeskalation der antimikrobiellen Therapie bei schweren Infektionen stehen nur begrenzt zur Verfügung [206]. Eine frühzeitige Deeskalation der antimikrobiellen Therapie im Kontext einer Kombinationstherapie ist bisher noch nicht untersucht worden. Beobachtungsstudien haben gezeigt, dass eine frühzeitige Deeskalation einer Kombinationstherapie mit äquivalenten oder überlegenen Ergebnissen bei Sepsis und septischem Schock verbunden ist [83, 207, 208], wobei mindestens eine Studie auf eine erhöhte Häufigkeit einer Superinfektion und längere Aufenthalte auf der ITS hinwies [209]. Eine frühzeitige Deeskalation kann zu Vorteilen für den einzelnen Patienten führen [210-212]. Obwohl die Daten nicht vollumfänglich konsistent sind, spricht vieles für eine frühzeitige Deeskalation bei der Nutzung einer Kombinationstherapie.

Während wesentliche Übereinstimmung dahingehend besteht, dass ein Bedarf für eine frühzeitige Deeskalation einer Kombinationstherapie vorhanden ist, mangelt es an einer Übereinkunft bezüglich präziser Kriterien für eine Deeskalation. Ansätze, die genutzt werden können, sind u. a. a) klinische Besserung (Abklingen des Schocks, Rückgang des Vasopressorbedarfs etc.), b) Rückgang von Infektionszeichen (insbesondere Prokalzitonin) und c) eine festgelegte Dauer der Kombinationstherapie.

\begin{tabular}{l}
\hline D.9 | Empfehlung | 2018 \\
\hline - Wir schlagen eine antimikrobielle \\
Behandlungsdauer von 7-10 Tagen \\
für die meisten Infektionen, die \\
mit Sepsis oder septischem Schock \\
assoziiert sind, vor. \\
- SSC-Leitlinienadaptation \\
- Konsensstärke: $92 \%$ \\
- Empfehlungsgrad: schwach \\
- Evidenzgrad: niedrig
\end{tabular}

\section{D.10 | Empfehlung | 2018}

- Wir empfehlen eine längere Behandlungsdauer (mind. 14 Tage) für Patienten mit Staphylococcus-aureusBakteriämie.

- SSC-Leitlinienadaptation; Abweichung des Empfehlungsgrads

- Konsensstärke: $100 \%$

- Empfehlungsgrad: stark

- Evidenzgrad: moderat

\section{D.11 | Empfehlung | 2018}

- Wir schlagen eine längere Behandlungsdauer für Patienten mit verzögertem Therapieansprechen, mit persistierenden Infektionsherden, mit bestimmten Pilz- und Virusinfektionen oder mit Immundefizienz einschließlich Neutropenie vor.

- SSC-Leitlinienadaptation und SSCLeitlinienmodifikation

- Konsensstärke: $100 \%$

- Empfehlungsgrad: schwach

- Evidenzgrad: niedrig

\section{D.12 | Empfehlung | 2018}

- Wir schlagen eine kürzere Behandlungsdauer bei Patienten mit schnellem klinischem Therapieansprechen, nach einer effektiven Fokuskontrolle bei intraabdomineller oder urogenitaler Sepsis und bei anatomisch unkomplizierter Pyelonephritis vor.

- SSC-Leitlinienadaptation

- Konsensstärke: $100 \%$

- Empfehlungsgrad: schwach

- Evidenzgrad: niedrig

\section{D.13 | Empfehlung | 2018}

- Expertenkonsens
- Wir empfehlen eine tägliche Beur-
teilung hinsichtlich der Deeskalation
der antimikrobiellen Therapie bei
Patienten mit Sepsis und septischem
Schock.
- SSC-Leitlinienadaptation
- Konsensstärke: $100 \%$

Begründung. Die unnötige therapeutische Nutzung von antimikrobiellen Substanzen trägt zur Entstehung und Verbreitung von Antibiotikaresistenzen bei [213]. Beim einzelnen Patienten kann sie Erkrankungen wie z.B. eine C.-difficileKolitis auslösen [212] und ist mit einem erhöhten Mortalitätsrisiko assoziiert, für das es noch keine hinreichenden wissenschaftlichen Erklärungen gibt [83].

Obwohl Patientenfaktoren die Länge der Antibiotikatherapie beeinflussen, ist eine Behandlungsdauer von 7-10 Tagen im Allgemeinen für die schwersten Infektionen angemessen [133, 214-216]. Die aktuellen Richtlinien empfehlen eine 7-tägige Behandlungsdauer bei nosokomialer Pneumonie (sowohl bei einer im Krankenhaus erworbenen als auch bei einer beatmungsassoziierten Pneumonie [VAP]; [133]). Aktuelle Daten deuten darauf hin, dass einige schwere Infektionen sogar noch kürzer behandelt werden können, insbesondere, wenn eine Fokussanierung erfolgreich umgesetzt werden konnte [217, 218]. Bei abdomineller Sepsis zeigte eine Subgruppenanalyse schwerstkranker Patienten (Acutephysiologic-and-chronic-health-evaluation[APACHE]-II-Score größer als 15 oder 20) keine Nachteile einer Kurzzeitbehandlung mit Antibiotika [217, 219]. Eine Behandlungsdauer von 3-5 Tagen oder eine noch kürzere Behandlungsdauer waren ebenso wirksam wie eine Behandlungsdauer von bis zu 10 Tagen. Des Weiteren haben Studien gezeigt, dass eine Behandlungsdauer von $<7$ Tagen ebenso wirksam ist wie eine längere Behandlungsdauer, was das Management einer akuten Pyelonephritis mit oder ohne Bakteriämie [218], einer unkomplizierten Zellulitis [220] und einer spontanen bakteriellen Peritonitis betrifft [221]. Bei manchen Erkrankungen ist eine längere antimikrobielle Therapie erforderlich. Dazu gehören ein langsames klinisches Ansprechen, nichtdrainierbare Infektionsherde, eine Bakteriämie mit Staph. aureus (einschließlich MRSA; [96, 134]), eine Candidämie bzw. eine invasive Candidiasis [135] und andere Pilzinfektionen, einige Virusinfektionen (z. B. Herpes, Zytomegalievirus) und Immundefizite einschließlich Neutropenie [222]. 
Die Art des infizierenden Pathogens spielt ebenfalls eine Rolle. Eine unkomplizierte Staph.-aureus-Bakteriämie erfordert eine mindestens 14-tägige Therapie, während die Behandlungsdauer für eine komplizierte Bakteriämie als endovaskuläre Infektion bei 6 Wochen liegt. Eine unkomplizierte Bakteriämie ist folgendermaßen definiert: 1) Ausschluss einer Endokarditis; 2) keine implantierten Prothesen; 3) negative Kontrollblutkulturen, die 2-4 Tage nach dem initialen Blutkulturset entnommen wurden; 4) Entfieberung innerhalb von $72 \mathrm{~h}$ nach dem Ansetzen einer effektiven Antibiotikatherapie und 5) kein Nachweis einer metastasierenden Infektion [134]. Patienten mit Candidämie (entweder katheterassoziiert oder nicht) und tiefen Kandidainfektionen, die entweder mit einer Sepsis einhergehen oder nicht, benötigen eine längere Therapie $[135,223]$. Hochresistente gramnegative Pathogene mit einer marginalen Sensitivität gegenüber den verwendeten Antimikrobiotika werden nur langsam eliminiert. Die Art und die Lokalisation der Infektion kann ebenfalls die Therapiedauer beeinflussen. Größere Abszesse und Osteomyelitis verfügen über eine begrenzte Medikamentenpenetration und erfordern eine längere Therapie. Obwohl allgemein bekannt ist, dass eine Endokarditis eine längere antimikrobielle Therapie erfordert, zeigt sich eine schwere Erkrankung typischerweise eher als Herzinsuffizienz/kardiogener Schock und Embolie als in Form einer Sepsis oder eines septischen Schocks [224]. Eine Vielzahl an anderen Faktoren kann die optimale Therapiedauer beeinflussen. Im Zweifelsfall sollte ein infektiologisches Konsil veranlasst werden.

Nur wenige Studien wurden dezidiert bei Patienten mit septischem Schock und Sepsis durchgeführt. Die Standardempfehlungen zur Therapiedauer beruhen in der Regel auf Studien mit weniger schwer kranken Kohorten.

Es gibt viele Gründe für eine unnötig lange antimikrobielle Therapie. Bei komplizierten, kritisch erkrankten Patienten, die mit schweren Infektionen aufgenommen werden, können eine nichtinfektiöse gleichzeitige Erkrankung und medizinische Eingriffe Anzeichen und Symptome hervorrufen, die im Einklang mit einer aktiven Infektion stehen (auch nach der Kontrolle der Infektion). Beispielsweise können Lungeninfiltrate und Kurzatmigkeit durch Lungenödeme verursacht werden, die neben einer Pneumonie bestehen, eine erhöhte Anzahl an weißen Blutkörperchen kann infolge der Verabreichung von Kortikosteroiden oder nach physiologischem Stress auftreten und Fieber kann mit bestimmten Medikamenten im Zusammenhang stehen, zu denen $\beta$-Laktame und Phenytoin gehören. Wie jedoch bereits dargestellt wurde, stellen Antimikrobiotika keine harmlose Therapieform dar. Bei Niedrigrisikopatienten können die Nebenwirkungen einen etwaigen Nutzen überwiegen. Angesichts des potenziellen Schadens, der mit unnötig langen antimikrobiellen Therapien verbunden sein kann, wird eine tägliche Beurteilung hinsichtlich einer Deeskalation der antimikrobiellen Therapie bei Patienten mit Sepsis oder septischem Schock empfohlen. Studien haben gezeigt, dass eine tägliche Erwägung einer antimikrobiellen Deeskalation effektiv ist und mit einer Verbesserung der Mortalitätsraten verbunden sein kann [84, 234].

\section{D.14 | Empfehlung | 2018}

\section{- Wir empfehlen, Messungen des Prokalzitoninwerts vorzunehmen, um die Dauer der antimikrobiellen Therapie bei Sepsispatienten zu verkürzen. Ausgenommen davon sind Patienten mit Infektionen, die eine längere Therapie erfordern (D.7). - SSC-Leitlinienmodifikation; Abwei- chung des Empfehlungsgrads \\ - Konsensstärke: $100 \%$ \\ - Empfehlungsgrad: stark \\ - Evidenzgrad: moderat}

Begründung. Die Nutzung von Galactomannan und $\beta$-D-Glucan ist bei der Beurteilung von invasivem Aspergillus (und einem breiten Spektrum von Pilzpathogenen) etabliert [226, 227]. Ebenso werden Messungen des Serumprokalzitonins in vielen Teilen der Welt ergänzend genutzt, um eine akute Infektion zu diagnostizieren und die Dauer der antimikrobiellen Therapie festzulegen. Diverse prokalzitoninbasierte Algorithmen werden verwendet, um die Deeskalation der antimikrobiellen Therapie bei schweren Infektionen und Sepsis zu steuern [228-233]. Es ist jedoch nicht erkennbar, dass ein bestimmter Algorithmus einen klinischen Vorteil gegenüber einem anderen Algorithmus besitzt. Ein großer Teil der Literatur legt nahe, dass die Nutzung derartiger Algorithmen die sichere antimikrobielle Deeskalation im Vergleich zu klinischen Standardansätzen ohne negative Auswirkungen auf die Mortalität beschleunigen kann. Vor Kurzem hat eine große randomisierte Studie zur Prokalzitoninnutzung bei kritisch kranken Patienten mit vermuteter bakterieller Infektion eine Reduzierung der Behandlungsdauer mit Antibiotika nachgewiesen [234]. In Anbetracht des Studiendesigns könnte die Reduzierung jedoch auch im Zusammenhang mit einem „soufflierten“ Effekt stehen, der bereits bei anderen Studien beobachtet wurde [84, 235]. Des Weiteren zeigte die Prokalzitoningruppe eine signifikante Reduzierung der Mortalität. Dieses Ergebnis steht im Einklang mit Studien, die einen Zusammenhang zwischen einer frühzeitigen antimikrobiellen Deeskalation und der Überlebensrate im Rahmen von Beobachtungsstudien zu Sepsis und septischem Schock demonstriert haben [83, 84]. Dieser Vorteil ist jedoch als ungesichert zu betrachten, da eine andere Metaanalyse von RCT zur Deeskalation keinen ähnlichen Vorteil in Bezug auf die Überlebensrate nachweisen konnte [236].

Es gilt nicht als erwiesen, dass die Nutzung von Prokalzitonin das Risiko von antibiotikaverbundener Diarrhö durch C. difficile reduziert. Es ist jedoch bekannt, dass das Auftreten einer C.difficile-Kolitis im Zusammenhang mit der kumulativen Antibiotikaexposition bei individuellen Patienten steht [212], sodass ein derartiger Vorteil wahrscheinlich ist. Obwohl bisher nicht nachgewiesen wurde, dass die Prävalenz einer antimikrobiellen Resistenz durch die Nutzung von Prokalzitonin reduziert wird, ist bekannt, dass die Entwicklung einer antimikrobiellen Resistenz im $\mathrm{Zu}$ - 
sammenhang mit dem Gesamtverbrauch von Antiinfektiva steht [213].

Es ist wichtig zu beachten, dass Prokalzitonin und alle anderen Biomarker nur unterstützende und ergänzende Daten zur klinischen Beurteilung zur Verfügung stellen können. Entscheidungen bezüglich des Ansetzens, der Änderung oder des Absetzens einer antimikrobiellen Therapie sollten niemals ausschließlich auf Grundlage von Veränderungen von Biomarkern (einschließlich Prokalzitonin) getroffen werden.

\section{E. Fokuskontrolle}

\section{E.1 | Empfehlung | 2018}

\section{- Expertenkonsens}

- Wir empfehlen, dass nach einem sanierbaren Fokus, einschließlich Fremdmaterialien, schnell und gezielt gesucht wird und ggf. eine operative/interventionelle Fokussanierung so schnell wie medizinisch und logistisch möglich erfolgen soll. - SSC-Leitlinienadaptation - Konsensstärke: $100 \%$

Begründung. Fundamentale Prinzipien der Fokuskontrolle beinhalten die schnelle Identifikation des Infektionsherds und dessen potenzielle chirurgische oder interventionelle Sanierung (insbesondere Abszessdrainage, Débridement von infiziertem nekrotischem Gewebe, Entfernung von potenziell infizierten Devices; [237]). $\mathrm{Zu}$ den Infektionsherden, die gut auf Fokuskontrollmaßnahmen ansprechen, gehören intraabdominelle Abszesse, gastrointestinale Perforationen, Darmischämie oder -volvulus, Cholangitis, Cholezystitis, mit einer Obstruktion oder einem Abszess verbundene Pyelonephritis, eine nekrotisierende Weichteilinfektion, andere tiefe Infektionen (z.B. Empyem oder septische Arthritis) sowie Infektionen von Implantaten.

Potenzielle Infektionsherde sollten so schnell wie möglich nach einer initialen Volumentherapie saniert werden [238, 239]. Ein Zeitfenster von nicht mehr als
6-12 h nach der Diagnose scheint in den meisten Fällen auszureichend zu sein.

Die klinische Erfahrung hat gezeigt, dass sich auch bei schwerstkranken Patienten eine Stabilisierung nur durch eine angemessene Fokuskontrolle erreichen lässt und nicht durch eine Erweiterung von supportiven oder antimikrobiellen Maßnahmen allein [138].

Bei der Auswahl von geeigneten Maßnahmen zur Fokuskontrolle müssen die Vorteile und Risiken einer spezifischen Intervention, das mit der Intervention verbundene Transportrisiko und die Erfolgswahrscheinlichkeit der betreffenden Maßnahme abgewogen werden. Die Interventionen können weitere Komplikationen verursachen wie z.B. Blutungen, Fisteln oder versehentliche Organverletzungen. Im Allgemeinen sollte die am wenigsten invasive Option angestrebt werden. Ein offener chirurgischer Eingriff sollte in Betracht gezogen werden, wenn andere Interventionsansätze nicht geeignet sind oder nicht zeitgerecht zur Verfügung stehen. Eine chirurgische Exploration kann auch indiziert sein, wenn der Infektionsherd trotz erfolgter Bildgebung nicht gesichert werden kann.

\section{E.2 | Empfehlung | 2018}

\section{- Expertenkonsens \\ - Wir empfehlen das unverzügliche Entfernen intravaskulärer Zugänge, die einen möglichen Ausgangspunkt für die Sepsis oder den septischen Schock darstellen. \\ - SSC-Leitlinienadaptation \\ - Konsensstärke: $100 \%$}

Begründung. Intravasale Zugänge wie zentrale Venenkatheter können Ausgangspunkt einer Sepsis oder eines septischen Schocks sein. Ein intravasaler Zugang sollte im Allgemeinen unverzüglich entfernt werden, nachdem ein anderer intravasaler Zugang gelegt wurde. Patienten mit implantierten, getunnelten Kathetern können mit einer längeren antimikrobiellen Therapie behandelt werden, falls kein septischer Schock oder keine Fungämie vorliegt und eine Entfernung des Katheters nicht praktikabel ist. Eine Entfernung des
Katheters ist primär jedoch immer anzustreben.

\section{F. Flüssigkeitstherapie}

\section{F.1 | Empfehlung | 2018}

\section{- Expertenkonsens \\ - Wir empfehlen, dass eine Flüssig- keitstherapie nach dem „Fluid- challenge"-Prinzip angewendet wird, bei der die Flüssigkeitsgabe fortge- setzt wird, solange Zeichen einer Hypoperfusion vorliegen. \\ - SSC-Leitlinienmodifikation \\ - Konsensstärke: $100 \%$}

Begründung. Die intravenöse Volumentherapie ist ein Eckpfeiler der modernen Therapie. Dennoch ist nur wenig Evidenz aus RCT verfügbar, die diese Praxis unterstützen würde. Eine Studie an (meist an Malaria erkrankten) Kindern in Afrika in einer Umgebung, in der andere supportive Maßnahmen nur sehr begrenzt verfügbar sind, stellte diese Praxis infrage [240]. Wir glauben jedoch, dass eine Extrapolation dieser Daten in Bezug auf Patienten in Regionen mit besserer medizinischer Versorgung nicht gerechtfertigt ist, und empfehlen daher, eine Euvolämie mit intravenösen Flüssigkeiten wiederherzustellen. Dieses sollte initial rasch erfolgen und dann zurückhaltend, sobald sich der Patient stabilisiert. Eine anhaltend positive Flüssigkeitsbilanz auf der Intensivstation ist mit einem schlechteren Outcome verbunden [241-245]. Daher sollte über die initiale Volumentherapie nur weiter Volumen verabreicht werden, wenn davon auszugehen ist, dass der Patient davon profitieren wird.

Abweichend von der SSC-Leitlinie wird als Ziel der weiterführenden Volumentherapie nicht die fortgesetzte Optimierung der hämodynamischen Faktoren, sondern die Vermeidung einer Hypoperfusion in den Vordergrund gestellt.

\section{F.2 | Empfehlung | 2018}

\section{- Wir empfehlen Kristalloide als initiale Flüssigkeitstherapie erster Wahl und}


eine anschließende intravaskuläre Volumenersatztherapie bei Patienten mit Sepsis und septischem Schock.

- SSC-Leitlinienadaptation

- Konsensstärke: $100 \%$

- Empfehlungsgrad: stark

- Evidenzqualität: moderat

Begründung. Beim Fehlen von eindeutigen Vorteilen einer Verabreichung von kolloidalen Volumenersatzlösungen im Vergleich zu kristalloiden Lösungen und in Anbetracht hoher Kosten für Albuminlösungen wird eine starke Empfehlung für eine Verwendung von kristalloiden Volumenersatzlösungen in der initialen Flüssigkeitstherapie von Patienten mit Sepsis und septischem Schock ausgesprochen.

\section{F.3 | Empfehlung | 2018}

\section{- Wir empfehlen die Verwendung von balancierten Kristalloiden für die Flüssigkeitstherapie von Patienten mit Sepsis oder septischem Schock. 0,9\%ige Kochsalzlösung sollte nicht verwendet werden. \\ - SSC-Leitlinienmodifikation; Abwei- chung des Empfehlungsgrads \\ - Konsensstärke bei Doppelabstim- mung: $100 \%$ \\ - Empfehlungsgrad: stark \\ - Evidenzqualität: moderat}

Begründung. Zur Fragestellung, welche kristalloide Lösung zur Flüssigkeitstherapie bei Patienten mit Sepsis oder septischen Schock besser geeignet ist, sind bislang nur wenige Daten aus klinischen Studien vorhanden. Yunos et al. untersuchten in zwei prospektiven Observationsstudien in einem Prä-/Post-Design die Auswirkungen eines Chlorid-basierten Infusionsregimes mit den Auswirkungen eines Chloridrestriktiven Infusionsregimes bei kritisch kranken Intensivpatienten [246, 247]. In diesen Studien wurden 1567 bzw. 2994 Intensivpatienten eingeschlossen. Die Verwendung eines chloridrestriktiven Regimes konnte in beiden Studien das Auftreten eines „acute kidney injury“ (AKI) im Stadiums 2 und 3 sowie die Notwendigkeit ei- ner Nierenersatztherapie signifikant vermindern, während sich bezüglich der Krankenhaussterblichkeit oder der Liegedauer auf der ITS keine Unterschiede zeigten. Eine aktuelle RCT zum Vergleich von balancierten Lösungen (Ringer-Lösung oder Plasma-Lyte-A-Lösung) mit 0,9\%igem $\mathrm{NaCl}$ an insgesamt 15.802 kritisch kranken Patienten zeigte bezüglich einem kombinierten Endpunkt (Sterblichkeit, neue Nierenersatztherapie und anhaltende Nierenfunktionsstörung) signifikante Vorteile. Insbesondere in der Subgruppe mit Sepsis oder septischem Schock (ca. 15\% aller Patienten) zeigte sich ein signifikant besseres Ergebnis für die balancierten Lösungen (OR 0,8; KI 0,67-0,94, $p=0,01$; [248]). Demgegenüber steht eine Metaanalyse von allerdings minderer Qualität von Rochwerg et al., die keinen Unterschied zwischen balancierten kristalloiden Lösungen und 0,9\%iger NaCl-Lösung bezüglich Sterblichkeit und Indikation zur Nierenersatztherapie aufzeigt [249]. In einer weiteren Studie (SPLIT-Trial) mit 2278 Intensivpatienten konnten keine positiven Effekte einer balancierten Lösung im Vergleich zu einer 0,9\%igen NaCl-Lösung gefunden werden. Diese Studie ist bezüglich der Aussagefähigkeit bei Sepsis jedoch stark eingeschränkt, da lediglich 3,7\% $(n=84)$ der Patienten eine Sepsis aufwiesen [250]. Inkludiert man die aktuelle Studie von Semler et al. in die Evidenztabelle, so ergibt sich bezüglich der Sterblichkeit kein Unterschied zwischen den beiden Lösungen (OR 0,89; KI 0,74-1,04) bezüglich des Auftretens einer schweren Nierenschädigung. Insgesamt besteht ein Vorteil für die balancierten Lösungen (OR 0,85; KI 0,74-0,98) auf moderatem Evidenzniveau [251].

Der Empfehlungsgrad „starke Empfehlung “ für balancierte Lösungen trägt dem Umstand Rechnung, dass zwar im Hinblick auf den isolierten Endpunkt Sterblichkeit für Patienten mit Sepsis und septischem Schock kein positiver Effekt gezeigt werden konnte, aber bei kombinierten Studienendpunkten und bezüglich der Vermeidung oder Verminderung von Organdysfunktion (Niere) die erwiesenen positiven Effekte der balancierten kristalloiden Lösungen überzeugen.

\section{F.4 | Empfehlung | 2018}

- Wir schlagen vor, dass von einer Verwendung von Albumin oder Gelatine bei der Behandlung von Patienten mit septischem Schock abgesehen wird, sofern eine adäquate Flüssigkeitstherapie mit Kristalloiden in der Lage ist, die hämodynamische Stabilität zu erreichen. Für den Fall, dass dies nicht möglich sein sollte, schlagen wir den ergänzenden Einsatz von Albumin oder Gelatine vor.

- SSC-Leitlinienmodifikation

- Konsensstärke bei Doppelabstimmung: $100 \%$

- Empfehlungsgrad: schwach

- Evidenzqualität: niedrig

Begründung. $\mathrm{Zu}$ der Fragestellung, ob kolloidale Lösungen in Kombination mit einer kristallinen Lösung als Flüssigkeitstherapie bei Patienten mit Sepsis oder septischem Schock vorteilhaft sind, liegen nur wenige Daten aus klinischen Studien vor. Die Ergebnisse einer RCT [252] zeigen einen Vorteil von Kolloiden inklusive von HES in der 90-Tage-Sterblichkeit auf. Da jedoch als primäres Studienziel der CRISTAL-Studie ein Unterschied in der 28-Tage-Sterblichkeit definiert worden war, handelt es sich um ein methodisch negatives Ergebnis. Metaanalysen konnten zeigen, dass auch der Einsatz von Gelatine im Vergleich zu Kristalloiden/HES/Albumin nicht mit einem erhöhten Mortalitätsrisiko oder einem erhöhten Risiko für eine Nierendysfunktion assoziiert ist. In einer RCT war die Gabe von Gelatine im Vergleich zu $6 \%$ HES 200/0,62 bei septischen Patienten mit weniger renaler Dysfunktion assoziiert. $4 \%$ Albumin war bei septischen Patienten in einer australischen Studie nichtsignifikant $(p=0,09)$ besser als $0,9 \%$ $\mathrm{NaCl}$.

Es ist also kaum Evidenz für den Einsatz von Albumin oder Gelatine vorhanden. Hierzu sind die Ergebnisse von laufenden großen RCT mit Albumin und Gelatine bei septischen Patienten abzuwarten (z.B. GENIUS-Studie, NCT02715466).

Angesichts der schwachen Beweislage für oder gegen den Einsatz von Al- 
bumin und/oder Gelatine ermöglicht die Änderung der Empfehlung F.4 Intensivmedizinern die Entscheidung für den zusätzlichen kolloidalen Volumenersatz mit Gelatine oder Albumin bei kritisch kranken und septischen Patienten. Diese Empfehlung mit dem Empfehlungsgrad „schwache Empfehlung“ für Albumin und/oder Gelatine trägt dem Umstand Rechnung, dass zwar im Hinblick auf den isolierten Endpunkt Sterblichkeit für die Gruppe der Patienten mit Sepsis und septischem Schock keine positiven Effekte gezeigt werden konnten, aber in der CRISTAL-Studie bei der Verwendung von Kolloiden in dieser Phase ein signifikanter Outcomevorteil nach $90 \mathrm{Ta}$ gen aufgezeigt werden konnte; dieses vor dem Hintergrund, dass in der Intensivmedizin die schnelle Stabilisierung der Hämodynamik kritisch kranker Patienten mit Schock innerhalb der ersten $6 \mathrm{~h}$ outcomerelevant ist [252].

\section{F.5 | Empfehlung | 2018}

\section{- Wir empfehlen, dass von der Verwen- dung von Hydroxyethylstärke zur intravaskulären Volumenersatzthe- rapie bei Patienten mit Sepsis oder septischem Schock abgesehen wird. \\ - SSC-Leitlinienadaptation \\ - Konsensstärke bei Doppelabstim- mung: $100 \%$ \\ - Empfehlungsgrad: stark \\ - Evidenzqualität: hoch}

Begründung. HES-Lösungen sind Kolloide, bei denen Sicherheitsbedenken bei Patienten mit Sepsis bestehen. Eine Metaanalyse von 9 Studien (3456 Patienten), in denen 6\%iges HES 130/0,38-0,45 mit Kristalloiden oder Albumin bei Patienten mit Sepsis verglichen wurden, ergab keinen Unterschied in Bezug aufdie Mortalität aller Ursachen (RR 1,04; 95\%-KI 0,89-1,22; [253]). Wurden jedoch diese Studien mit geringem Verzerrungspotenzial separat analysiert, führte die Nutzung von HES zu einem höheren Sterberisiko im Vergleich zu anderen Flüssigkeiten (RR 1,11; 95\%-KI 1,01-1,22; hohe Evidenzqualität). Dies entspricht 34 zusätzlichen Todesfällen pro 1000 Patienten. Des Weiteren führte die HES-Nutzung zu einem höheren Risiko für eine Behandlung mit Nierenersatztherapien (RR 1,36; $95 \%$-KI 1,08-1,72; hohe Evidenzqualität).

Eine anschließende Metaanalyse konzentrierte sich auf die akute Flüssigkeitstherapie bei Patienten mit Sepsis oder septischem Schock und ergab, dass HES zu einem höheren Sterberisiko (10 RCT; OR 1,13; CrI 0,99-1,30; hohe Evidenzqualität) und Bedarf einer Nierenersatztherapie (7 RCT; OR 1,39; CrI 1,17-1,66; hohe Evidenzqualität) im Vergleich zu Kristalloiden führte. Beim Vergleich von Albumin mit HES führte Albumin zu einem geringeren Sterberisiko (OR 0,73; CrI 0,56-0,93; mittlere Evidenzqualität) und zu einer Tendenz in Richtung eines geringeren Bedarfs an Nierenersatztherapien (OR 0,74; CrI 0,53-1,04; geringe Evidenzqualität; [249]). Die unerwünschten Folgen der Verwendung von HES (erhöhtes Sterberisiko und Bedarf für eine Nierenersatztherapie) und die mittlere bis hohe Qualität der entsprechenden Evidenz begründen die starke Empfehlung gegen die Nutzung von HES in der Flüssigkeitstherapie von Patienten mit Sepsis oder septischem Schock.

\section{G. Vasoaktive Medikation}

Neben der Flüssigkeitstherapie stellt die Anwendung von vasoaktiven Substanzen eine wichtige Strategie in der raschen hämodynamischen Stabilisierung bei Sepsis und septischen Schock dar. Die physiologischen Effekte von Vasopressoren und einer kombinierten Auswahl von inotropen Medikamenten und Vasopressoren bei septischem Schock wurden in einer großen Anzahl von Literaturreviews beschrieben.

\section{G.1 | Empfehlung | 2018}

\section{- Wir empfehlen Noradrenalin als Vasopressor erster Wahl. \\ - SSC-Leitlinienadaptation \\ - Konsensstärke: $100 \%$ \\ - Empfehlungsgrad: stark \\ - Evidenzgrad: moderat}

Begründung. Die physiologischen Effekte von Vasopressoren und einer Kom- bination von inotropen Medikamenten und Vasopressoren bei septischem Schock wurden in mehreren Reviews beschrieben [254-263, 686].

Norepinephrin erhöht den MAP aufgrund seiner vasokonstriktiven Wirkungen bei geringen Veränderungen der Herzfrequenz und einer geringeren $\mathrm{Zu}$ nahme des Schlagvolumens im Vergleich zu Dopamin. Dopamin erhöht den MAP und das Herzzeitvolumen (HZV), was hauptsächlich auf eine Erhöhung des Schlagvolumens und der Herzfrequenz zurückzuführen ist. Norepinephrin ist potenter als Dopamin und kann bei der Behandlung von Hypotonie bei Patienten mit septischem Schock wirksam sein. Bei Patienten mit beeinträchtigter systolischer Funktion kann Dopamin besonders hilfreich sein, wobei es jedoch mehr Tachykardien verursacht und arrhythmogener zu sein scheint als Norepinephrin [264]. Es kann zudem die endokrine Reaktion über die Hypothalamus-Hypophysen-Achse beeinflussen und immunsuppressive Effekte haben [265]. Ein aktuelles systematisches Review und eine Metaanalyse, die 11 randomisierte Studien umfassten $(n=1710)$ und einen Vergleich von Norepinephrin mit Dopamin beinhalteten, befürworteten die Verwendung von Dopamin bei der Behandlung von septischem Schock nicht [266]. Tatsächlich führte die Nutzung von Norepinephrin zu einer verringerten Mortalität (RR 0,89; $95 \%$ KI 0,81-0,98, hohe Evidenzqualität) und einem geringeren Risiko für $\mathrm{Ar}$ rhythmien (RR 0,48; $95 \%$-KI 0,40-0,58; hohe Evidenzqualität) im Vergleich zu Dopamin.

Studien deuten darauf hin, dass eine Epinephrininfusion schädliche Auswirkungen auf die Durchblutung des Splanchnikusgebiets haben und eine Hyperlaktatämie verursachen könnte. Klinische Studien haben jedoch keine Verschlechterung der klinischen Ergebnisse nachgewiesen. Eine RCT, in der Norepinephrin mit Epinephrin verglichen wurde, ergab keinen Unterschied in der Mortalität, wies aber eine Zunahme der medikamentenbezogenen Nebenwirkungen bei der Nutzung von Epinephrin nach [267]. Auch eine Metaanalyse von 4 randomisierten Studien $(n=540)$, in 
denen Norepinephrin mit Epinephrin verglichen wurde, wies keinen signifikanten Unterschied in Bezug auf die Mortalität nach (RR 0,96; KI 0,77-1,21; niedrige Evidenzqualität; [266]). Epinephrin kann die aerobe Laktatproduktion erhöhen, indem es die adrenergen Skelettmuskel- $\beta_{2}$-Rezeptoren stimuliert, und könnte auf diese Weise die Nutzung der Laktatclearance ausschließen, was die Steuerung der Flüssigkeitstherapie betrifft.

\section{G.2 | Empfehlung | 2018}

\section{- Wir schlagen vor, dass entweder Vasopressin oder Epinephrin zu Norepinephrin ergänzt wird, wenn mit Noradrenalin allein kein ausrei- chender Blutdruck erzielt werden kann. \\ - SSC-Leitlinienmodifikation \\ - Konsensstärke: $100 \%$ \\ - Empfehlungsgrad: schwach \\ - Evidenzgrad: niedrig}

Begründung. Die Vasopressinserumkonzentrationen bei Patienten mit septischem Schock sind unangemessen erniedrigt.

Niedrige Vasopressindosen können bei Patienten, die einen refraktären Schock unter anderen Vasopressoren aufweisen, den Blutdruck steigern und weisen zusätzliche physiologische Vorteile auf [268-272]. Terlipressin hat eine ähnliche Wirkung, ist aber langanhaltend wirksam [273]. Studien zeigten, dass die Vasopressinplasmakonzentrationen im frühen septischen Schock erhöht sind, aber bei der Mehrheit der Patienten nach $24-48 \mathrm{~h}$ in den normalen Wertebereich zurückgehen, während der Schockzustand andauert [274]. Dieses Ergebnis wird als relative Vasopressindefizienz bezeichnet, da zu erwarten wäre, dass die Vasopressinkonzentration bei Vorliegen einer Hypotonie erhöht sein würde. Die klinische Bedeutung dieser Befunde ist unzureichend untersucht.

Die VASST-Studie, eine RCT, in der Norepinephrin als Monosubstanz mit Norepinephrin plus Vasopressin in einer Dosierung von $0,03 \mathrm{U} / \mathrm{min}$ verglichen wurde, ergab keinen Unterschied in Be- zug auf das Ergebnis in der Intent-totreat-Population [275]. Eine geplante A-priori-Subgruppenanalyse zeigte eine Verbesserung der Überlebensrate bei jenen Patienten, die bei Randomisierung Norepinephrin in einer Dosierung von $<15 \mu \mathrm{g} / \mathrm{min}$ zusätzlich zu Vasopressin erhielten. Die Rationale für diese Stratifizierung bei Studienplanung war jedoch auf einen potenziellen Nutzen bei den Patienten angelegt, die $\geq 15 \mu \mathrm{g} / \mathrm{min}$ Norepinephrin benötigten. Höhere Dosen von Vasopressin sind mit kardialer und Splanchnikusischämie verbunden und sollten Situationen vorbehalten bleiben, in denen alternative Vasopressoren versagt haben [269].

In der VANISH-Studie wurden 409 Patienten mit septischem Schock im Rahmen eines faktoriellen $(2 \times 2)$ Designs randomisiert der Behandlung mit Vasopressin in Kombination mit Placebo oder Hydrokortison oder Norepinephrin mit Placebo oder Hydrokortison zugewiesen. Es wurde kein signifikanter Unterschied in Bezug auf die Tage ohne Nierenversagen oder Tod festgestellt. Allerdings wurde in der Vasopressingruppe seltener eine Nierenersatztherapie durchgeführt [276]. Wir haben eine aktualisierte Metaanalyse durchgeführt, in der die Ergebnisse der VANISH-Studie einbezogen wurden. Daten aus 9 Studien $(n=1324$ Patienten mit septischem Schock), in denen Norepinephrin mit Vasopressin (oder Terlipressin) verglichen wurde, demonstrierten keinen signifikanten Unterschied in Bezug auf die Mortalität (RR 0,89; $95 \%$-KI 0,79-1,00; mittlere Evidenzqualität; [269, 272, 273, 277-279]). Die Ergebnisse fielen ähnlich aus, nachdem Studien ausgeschlossen wurden, die eine Kombination aus Norepinephrin und Vasopressin im Interventionsarm verwendet hatten (RR 0,89; $95 \%$-KI 0,77-1,02).

Es mangelt an großen Studien, in denen Vasopressin mit anderen Vasopressoren bei septischem Schock verglichen wurde. Die zu Vasopressin publizierten Daten unterstützen mehrheitlich einen Einspareffekt in Bezug auf die Norepinephrindosis. Im Hinblick auf den Effekt von Vasopressin auf die Mortalität besteht Unsicherheit. Norepinephrin bleibt daher der Vasopressor erster Wahl in der Behandlung von Patienten mit septischem Schock. Wir empfehlen nicht die Nutzung von Vasopressin als Erstlinienvasopressor für die Unterstützung des MAP und würden zur Vorsicht raten, wenn es bei Patienten genutzt wird, die nicht euvolämisch sind oder höhere Dosen als $0,03 \mathrm{U} / \mathrm{min}$ erhalten.

Phenylephrin ist ein reiner $\alpha$-adrenerger Agonist. Klinische Studiendaten zur Verwendung bei Sepsis stehen nur begrenzt zur Verfügung. Phenylephrin verfügt über das Potenzial, eine Splanchnikusvasokonstriktion $\mathrm{zu}$ verursachen [280]. Eine Netzwerkmetaanalyse ergab inkonsistente Ergebnisse (breite Konfidenzintervalle), wenn Phenylephrin mit anderen Vasopressoren verglichen wurde [281]. Daher ist die Auswirkung auf die klinischen Ergebnisse ungewiss und die Nutzung von Phenylephrin sollte eingeschränkt werden, bis mehr Forschungsergebnisse zur Verfügung stehen.

Abweichend von der SSC-Leitlinie wurde die Evidenz dieser Empfehlung als niedrig beurteilt.

\section{G.3 | Empfehlung | 2018}

\section{- Wir empfehlen, auf die Verwendung von Dopamin zu verzichten. \\ - Zusätzliche DSG-Leitlinienempfeh- lung \\ - Konsensstärke: $100 \%$ \\ - Empfehlungsgrad: stark \\ - Evidenzgrad: niedrig}

Begründung. Abweichend von der SSCLeitlinie interpretieren wir die vorliegenden Studien dahingehend, dass Dopamin im Vergleich mit Noradrenalin keine Vorteile hat. Es besteht ein erhöhtes Risiko von Rhythmusstörungen. Eine Empfehlung, den Einsatz auf Patienten mit einem geringen Risiko von Rhythmusstörungen zu beschränken, erscheint nicht sinnvoll. Konsequenterweise sollte auf den Einsatz von Dopamin komplett verzichtet werden. 


\section{G.4 | Empfehlung | 2018 \\ - Wir empfehlen, dass von einer Ver- wendung von Niedrigdosisdopamin zum Nierenschutz abgesehen wird. \\ - SSC-Leitlinienadaptation \\ - Konsensstärke: $100 \%$ \\ - Empfehlungsgrad: stark \\ - Evidenzgrad: hoch}

Begründung. Eine große randomisierte Studie und eine Metaanalyse, die Niedrigdosisdopamin mit einem Placebo verglichen hat, ergab keinen Unterschied in Bezug auf Arrhythmien, den Bedarf für eine Nierenersatztherapie, die Urinausscheidung, die Überlebensrate, die Dauer bis zur Erholung der Nierenfunktion und die Aufenthaltsdauer auf der ITS und im Krankenhaus [282, 687]. Somit unterstützen die verfügbaren Daten nicht die Verabreichung von niedrigen Dosen von Dopamin zum alleinigen Zweck der Aufrechterhaltung der Nierenfunktion.

\section{G.5 | Empfehlung | 2018}

\section{- Wir schlagen die Verwendung von Dobutamin bei Patienten vor, bei denen Nachweise für eine persis- tierende Hypoperfusion trotz an- gemessener Flüssigkeitszufuhr und des Einsatzes von Vasopressoren vorliegen. \\ - Bemerkungen: Sofern der Wirkstoff angesetzt wird, sollte die Dosis des Vasopressors auf einen Endpunkt titriert werden, der eine Perfusion widerspiegelt, und der Wirkstoff sollte reduziert oder abgesetzt werden, sofern eine Aggravierung der Hypotonie oder Arrhythmien auftreten. \\ - SSC-Leitlinienadaptation \\ - Konsensstärke: $100 \%$ \\ - Empfehlungsgrad: schwach \\ - Evidenzgrad: niedrig}

Begründung. Eine myokardiale Dysfunktion infolge einer Infektion kann bei einigen Patienten mit septischem Schock auftreten, aber das HZV wird üblicherweise durch eine ventrikuläre Dilatation, Tachykardie und einen redu- zierten vaskulären Widerstand aufrechterhalten [283]. Ein gewisser Anteil dieser Patienten kann über eine verringerte kardiale Reserve verfügen und daher nicht in der Lage sein, ein HZV zu erreichen, um ein angemessenes Sauerstoffangebot sicherzustellen. Die Erkennung einer derart reduzierten kardialen Reserve kann eine Herausforderung darstellen; bildgebende Verfahren, die eine verringerte Ejektionsfraktion zeigen, müssen nicht notwendigerweise auch ein unzureichendes HZV bedeuten. Begleitende Messungen des HZV in Kombination mit dem quantitativen Nachweis einer angemessenen Perfusion sind zu bevorzugen.

Eine routinemäßige Erhöhung des Herzausgabevolumens auf im Vorfeld festgelegte "supranormale“ Werte bei allen Patienten führt eindeutig nicht $\mathrm{zu}$ einer Verbesserung der Ergebnisse, wie in 2 großen prospektiven klinischen Studien an kritisch kranken ITS-Patienten mit Sepsis unter Behandlung mit Dobutamin gezeigt wurde [284-286].

Einige Patienten könnten jedoch von einer verbesserten Gewebeperfusion infolge einer inotropen Therapie, die auf eine Erhöhung des Sauerstoffangebots ausgerichtet ist, profitieren. In dieser Situation stellt Dobutamin für Patienten mit einem gemessenen oder vermuteten niedrigen $\mathrm{HZV}$, bei denen ein angemessener linksventrikulärer Füllungsdruck (oder die klinische Beurteilung einer angemessenen Flüssigkeitstherapie) sowie ein angemessener MAP vorliegt, die inotrope Therapie erster Wahl dar. Die beste Möglichkeit, diese Art von Therapie zu steuern, besteht darin, die Reaktion von Perfusionsindizes auf den (gemessenen) Anstieg des HZV zu überwachen [286].

Die Daten, die die Nutzung von Dobutamin unterstützen, sind hauptsächlich physiologischer Art und zeigen eine verbesserte Hämodynamik und einige Verbesserungen bei den Perfusionsindizes, die eine klinische Verbesserung, abnehmende Laktatspiegel und eine $\mathrm{S}_{\mathrm{cv}} \mathrm{O}_{2}$ Verbesserung umfassen können. RCT, die die Effekte von Dobutamin gegenüber einem Placebo im Hinblick auf die klinischen Ergebnisse verglichen haben, sind nicht bekannt. Die Mortalität bei Patienten, die randomisiert einer Therapie mit
Dobutamin zusätzlich zu Norepinephrin zugewiesen worden waren, wies keinen Unterschied im Vergleich zu einer Therapie mit Epinephrin auf [286], wobei die Studie aufgrund einer unzureichenden Patientenzahl nicht über genügend Aussagekraft verfügt. Dobutamin wurde ferner als inotrope Erstlinientherapie im Rahmen der Standardbehandlung in klinischen EGDT-Studien verwendet $[33,36,287,288]$ und negative Auswirkungen auf die Mortalität wurden bei dessen Verwendung nicht festgestellt.

\section{G.6 | Empfehlung | 2018}

\section{- Expertenkonsens \\ - Wir empfehlen, dass alle Patienten, die Vasopressoren benötigen, einen arteriellen Katheter erhalten, sobald dies praktisch realisierbar ist und sofern Ressourcen verfügbar sind. \\ - SSC-Leitlinienadaptation, Abwei- chung des Empfehlungsgrads - Konsensstärke: $100 \%$}

Begründung. In Schockzuständen kann die Schätzung des Blutdrucks unter Verwendung einer Manschette insbesondere bei automatisierten Messsystemen ungenau ausfallen. Die Nutzung eines arteriellen Zugangs ermöglicht eine genauere und besser reproduzierbare Messung des arteriellen Drucks $[286,289]$ und eine Schlag-zu-Schlag-Analyse, sodass Entscheidungen bezüglich der Therapie auf Grundlage von sofort verfügbaren und reproduzierbaren Blutdruckinformationen getroffen werden können [290]. Die Einführung von radial-arteriellen Kathetern ist im Allgemeinen sicher. Eine systematische Überprüfung von Beobachtungsstudien ergab, dass die Inzidenz von Ischämien der Gliedmaßen und von Blutungen bei weniger als $1 \%$ liegt, wobei es sich bei der häufigsten Komplikation um lokalisierte Hämatome handelt (14\%) [291]. Die Komplikationsraten könnten geringer ausfallen, wenn eine ultraschallgesteuerte Technik genutzt wird [292]. Ein systematisches Review zeigte im Vergleich zu radial-arteriellen Kathetern ein höheres Infektionsrisiko, wenn femoralarterielle Katheter genutzt wurden (RR 1,93; 95\%-KI 1,32-2,84), 
und die gepoolte Gesamtinzidenz von Blutkreislaufinfektionen betrug 3,4 pro 1000 Katheter [293]. Es besteht ein Mangel an großen randomisierten Studien, in denen die arterielle Blutdrucküberwachung mit nichtinvasiven Methoden verglichen wird.

In Anbetracht der geringen Komplikationsrate und der wahrscheinlich besseren Schätzung des Blutdrucks, aber auch unter Berücksichtigung der potenziell begrenzten Ressourcen in einigen Ländern und des Mangels an Studien hoher Qualität überwiegen wahrscheinlich die Vorteile von arteriellen Kathetern die Risiken. Daher sprechen die Mitglieder der SSC-Leitlinie eine schwache Empfehlung zugunsten der Positionierung von arteriellen Kathetern aus. Die arteriellen Katheter sollten entfernt werden, sobald keine hämodynamische Überwachung mehr benötigt wird, um das Komplikationsrisiko zu minimieren.

Abweichend von der SSC-Leitlinie wurde diese Empfehlung als Expertenkonsens beurteilt.

\section{Andere vasoaktive Medikamente}

Auch alternative inotrope Wirkstoffe können verwendet werden, um das $\mathrm{HZV}$ in spezifischen Situationen zu erhöhen. Phosphodiesteraseinhibitoren führen $\mathrm{zu}$ einer Erhöhung des intrazellulären zyklischen AMP und haben somit inotrope Effekte, die unabhängig von den adrenergen $\beta$-Rezeptoren sind. Der Phosphodiesteraseinhibitor Milrinon hat nachweislich das HZV in einer kleinen randomisierten Studie an 12 pädiatrischen Patienten erhöht, wobei die Studie in Bezug auf die Ergebnisanalyse nicht über genügend Aussagekraft verfügt [294]. Levosimendan erhöht die Kalziumresponsivität der kardialen Myozyten und öffnet außerdem die ATPabhängigen Kaliumkanäle, was dem Medikament sowohl inotrope als auch vasodilatatorische Eigenschaften verleiht. In Anbetracht der potenziellen Störung der myokardialen Kalziumsensitivität bei sepsisinduzierter Kardiomyopathie ist die Verwendung von Levosimendan auch bei septischem Schock vorgeschlagen worden.

\section{G.7| Empfehlung | 2018}

\section{- Wir empfehlen, Levosimendan zur hämodynamischen Stabilisierung bei septischen Patienten nicht anzuwen- den. \\ - Zusätzliche DSG-Leitlinienempfeh- lung \\ - Konsensstärke: $100 \%$ \\ - Empfehlungsgrad: stark \\ - Evidenzgrad: hoch}

Begründung. In einer Studie an $35 \mathrm{~Pa}$ tienten mit septischem Schock und akutem Atemnotsyndrom (ARDS), die randomisiert der Behandlung mit Levosimendan oder einem Placebo zugeordnet wurden, verbesserte Levosimendan die rechtsventrikuläre Leistung und die gemischtvenöse Sauerstoffsättigung im Vergleich zum Placebo [295]. Es sind nur wenige Studien zum Vergleich von Levosimendan mit Dobutamin vorhanden, die allerdings keinen eindeutigen Vorteil in Bezug auf Levosimendan demonstrieren [296]. Levosimendan ist teurer als Dobutamin und in vielen Teilen der Welt nicht verfügbar. Sechs kleine RCT (mit insgesamt 116 Patienten) verglichen Levosimendan mit Dobutamin, wobei die gepoolten Schätzungen keinen signifikanten Effekt auf die Mortalität zeigten (RR 0,83; 95\%-KI 0,66-1,05; niedrige Evidenzqualität). Angesicht der verfügbaren Evidenz niedriger Qualität und der höheren Kosten, die mit Levosimendan verbunden sind, bleibt Dobutamin das bevorzugte Medikament für diese Population. In einer RCT, in der 516 Patienten mit septischem Schock randomisiert entweder der Behandlung mit Levosimendan oder einem Placebo zugeordnet wurden, zeigte sich kein Unterschied in Bezug auf die Mortalität. Levosimendan führte jedoch $\mathrm{zu}$ einem signifikant erhöhten Risiko einer supraventrikulären Tachyarrhythmie im Vergleich zum Placebo (absoluter Unterschied 2,7\%; $95 \%$-KI 0,1-5,3\%; [297]). Die Ergebnisse dieser Studie stellen die systematische Verwendung dieses Wirkstoffs bei Patienten mit septischem Schock infrage. $\mathrm{Zu}$ beachten ist, dass die Herzfunktion in dieser Studie nicht beurteilt wurde und dass Patienten mit einem infolge einer Beeinträchtigung der Herzfunktion erniedrigten HZV von der inotropen Stimulation profitieren könnten.

\section{G.8 | Empfehlung | 2018}

\section{- Expertenkonsens \\ - Bezüglich der Behandlung von tachy- karden Rhythmusstörungen können $\beta_{1}$-selektive $\beta$-Blocker erwogen wer- den. \\ - Zusätzliche DSG-Leitlinienempfeh- lung}

- Konsensstärke: $100 \%$

Begründung. Sepsis und septischer Schock sind unter anderem durch eine exzessive adrenerge Stimulation charakterisiert, die zu Tachykardie, Rhythmusstörungen sowie $\mathrm{zu}$ systolischen und diastolischen Funktionsstörungen des Myokards führen können, die mit einer erhöhten Mortalität assoziiert sind [298, 299, 688]. Eine Applikation von $\beta$-Blockern könnte theoretisch der adrenergen Stimulation entgegenwirken und die Herzfunktion optimieren (Senkung des myokardialen Sauerstoffbedarfs, Reduktion der Tachykardie, verbesserte diastolische Füllung/Relaxation, Antiarrhythmitizität). Darüber hinaus gibt es Hinweise, dass $\beta$-Blocker als Modulatoren des Immunsystems eine wichtige Rolle spielen könnten [300].

$\beta$-Blocker werden bei Patienten mit Herzinsuffizienz und koronarer Herzkrankheit zur Verbesserung der myokardialen Funktion und zur Senkung des Reinfarktrisikos erfolgreich eingesetzt. Darüber hinaus können $\beta$-Blocker über eine Senkung der Herzfrequenz den myokardialen Sauerstoffbedarf senken. Aus diesen Überlegungen heraus kann der Einsatz von $\beta_{1}$-selektiven $\beta$-Blockern bei tachykarden Rhythmusstörungen auch in der Sepsis von Vorteil sein. Die Anwendung sollte unter entsprechendem hämodynamischem Monitoring titriert erfolgen [301-303].

\section{G.9 | Empfehlung | 2018}

\section{- Der routinemäßige Einsatz von $\beta$-Blo- ckern zur hämodynamischen Stabili-}


sierung bei septischen Patienten wird nicht empfohlen.

- Zusätzliche DSG-Leitlinienempfehlung

- Konsensstärke: $100 \%$

- Empfehlungsgrad: schwach

- Evidenzgrad: niedrig

Begründung. Für die routinemäßige Anwendung des $\beta_{1}$-selektiven $\beta$-Blockers Esmolol zeigte eine systematische Übersicht aus 10 qualitativ minderwertigen Studien (eine Metaanalyse war nicht durchführbar), dass Esmolol die Herzfrequenz senken kann. Weitere Effekte, insbesondere auf die Morbidität oder Mortalität, waren nicht erkennbar [304].

Im Gegensatz dazu zeigte eine prospektiv offene Studie von Morelli et al. eine signifikante Reduktion der Sterblichkeit in der $\beta_{1}$-Blocker-Gruppe [305]. Diese Studie ist jedoch mit erheblichen Einschränkungen $\mathrm{zu}$ bewerten, sodass sie lediglich ein „proof of principle“ der sicheren Herzfrequenzreduktion durch Esmolol darstellt. Trotzdem lassen die Ergebnisse dieser Studie einen Versuch der hämodynamischen Stabilisierung durch Frequenzsenkung unter bestimmten Bedingungen als gerechtfertigt erscheinen. Die aktuell vorhandene Literatur zu dieser Thematik ist widersprüchlich und die Evidenz für eine belastbare Aussage ist sehr gering, sodass zum jetzigen Zeitpunkt keine generelle Empfehlung für den routinemäßigen Einsatz von $\beta$-Blockern bei Sepsis oder septischen Schock gegeben werden kann. Die oben genannte "Proof-of-principle“-Studie (Morelli et al.) bedarf der Bestätigung in einer weiteren Studie.

\section{H. Kortikosteroide}

\section{H.1 | Empfehlung | 2018}

- Wir schlagen vor, dass von einer Verwendung von intravenösem Hydrokortison bei der Behandlung von Patienten mit septischem Schock abgesehen wird, sofern eine adäquate Flüssigkeitstherapie und hochdosierte Vasopressortherapie in der Lage sind, die hämodynamische Stabilität wiederherzustellen.

- Für den Fall, dass dies nicht erreichbar sein sollte, schlagen wir den Einsatz von intravenösem Hydrokortison in einer Dosis von $200 \mathrm{mg}$ pro Tag vor.

- SSC-Leitlinienadaptation

- Konsensstärke: $100 \%$

- Empfehlungsgrad: schwach

- Evidenzqualität: niedrig

Begründung. Die Reaktion von Patienten mit septischem Schock auf die Flüssigkeits- und Vasopressortherapie scheint ein wichtiger Faktor bei der Auswahl von Patienten für eine optionale Hydrokortisontherapie zu sein. Eine französische multizentrische RCT an $\mathrm{Pa}$ tienten mit septischem Schock, die nicht auf eine Vasopressortherapie ansprachen (systolischer Blutdruck $<90 \mathrm{~mm} \mathrm{Hg}$ trotz Flüssigkeits- und Vasopressortherapie über einen Zeitraum von mehr als einer Stunde), zeigte einen signifikanten Schockrückgang und eine Reduzierung der Mortalitätsrate bei Patienten mit relativer Nebenniereninsuffizienz (definiert als ein maximaler Anstieg des postadrenokortikotropen Hormons (ACTH) Kortisol $\leq 9 \mu \mathrm{g} / \mathrm{dl}$; [306]). Zwei kleinere RCT zeigten ebenfalls signifikante Effekte in Bezug auf einen Schockrückgang mit einer Steroidtherapie [307, 308]. Im Gegensatz dazu wies eine große europäische multizentrische Studie (CORTICUS), in die Patienten mit einem systolischen Blutdruck von $<90 \mathrm{~mm} \mathrm{Hg}$ trotz einer angemessenen Flüssigkeitsersatztherapie oder eines Bedarfs für Vasopressoren einbezogen wurden, ein geringeres Sterberisiko auf als die französische Studie und erbrachte keinen Nachweis für einen Mortalitätsvorteil bei Anwendung einer Hydrokortisontherapie [309]. Es lag kein Unterschied in Bezug auf die Mortalität in den Gruppen vor, die entsprechend der ACTH-Reaktion stratifiziert wurden.

Diverse systematische Reviews haben die Nutzung von Niedrigdosishydrokortison bei septischem Schock mit widersprüchlichen Ergebnissen untersucht. Annane et al. [306] analysierten die Ergebnisse von 12 Studien und errechneten eine signifikante Reduzierung der 28-tägigen Mortalität bei einer längeren
Niedrigdosisbehandlung bei erwachsenen Patienten mit septischem Schock (RR 0,84; 95\%-KI 0,72-0,97; $p=0,02$ ). Parallel dazu nutzten Sligl et al. [310] eine ähnliche Technik, identifizierten allerdings nur 8 Studien für ihre Metaanalyse, von denen 6 Studien über ein High-level-RCT-Design mit einem geringen Verzerrungspotenzial verfügten. Im Gegensatz zu dem zuvor erwähnten Review ergab diese Analyse keinen statistisch signifikanten Unterschied hinsichtlich der Mortalität (RR 1,00; $95 \%$ KI 0,84-1,18). Beide Reviews bestätigten jedoch eine Verbesserung in Bezug auf den Schockrückgang bei der Verwendung von Niedrigdosishydrokortison.

In jüngerer Zeit bezogen Annane et al. 33 geeignete Studien $(n=4268)$ in ein neues systematisches Review ein [306]. Von diesen 33 Studien wiesen 23 Studien ein geringes Auswahlverzerrungspotenzial auf; 22 Studien verfügten über ein geringes Performance- und Detektionsverzerrungspotenzial; 27 Studien hatten ein geringes Attritionsverzerrungspotenzial und 14 Studien verfügten über ein geringes Risiko hinsichtlich einer selektiven Berichterstattung. Kortikosteroide reduzierten die 28-Tage-Mortalität (27 Studien; $n=3176$; RR 0,87; $95 \%-\mathrm{KI}$ 0,76-1,00). Eine lange Behandlungseinheit mit Niedrigdosiskortikosteroiden reduzierte die 28-Tage-Mortalität signifikant (22 Studien; RR 0,87; 95\%-KI 0,78-0,97). Kortikosteroide reduzierten auch die ITS-Mortalität (13 Studien; RR 0,82; $95 \%$-KI 0,68-1,00) und die Krankenhausmortalität (17 Studien; RR 0,85; 95\%-KI 0,73-0,98). Kortikosteroide erhöhten den Anteil der Schockrückgänge zum Tag 7 (12 Studien; RR 1,31; 95 \%-KI 1,14-1,51) und zum Tag 28 (7 Studien; $n=1013$; RR 1,11; $95 \%$-KI 1,02-1,21). Und schließlich wurde ein zusätzliches systematisches Review von Volbeda et al. veröffentlicht, in das insgesamt 35 Studien mit 4682 randomisierten Patienten einbezogen wurden (mit Ausnahme von 2 Studien bestand bei allen Studien ein hohes Verzerrungspotenzial; [311]). Konträr wurde in diesem Review kein statistisch signifikanter Effekt auf die Mortalität bei allen untersuchten Steroiddosen im Vergleich zum Placebo festgestellt und auch bei 
keiner Intervention nach der maximalen Anzahl der Nachuntersuchungen. Die beiden Studien mit einem geringen Verzerrungspotenzial wiesen ebenfalls keinen statistisch signifikanten Unterschied nach (Zufallseffektmodell; RR 0,38; $95 \%$-KI 0,06-2,42). Ähnliche Ergebnisse wurden in Untergruppen von Studien festgestellt, die entsprechend der Nutzung von Hydrokortison (oder eines äquivalenten Medikaments) bei hoher Dosis $(>500 \mathrm{mg}$ ) oder niedriger Dosis $(\leq 500 \mathrm{mg})$ stratifiziert worden waren (RR 0,87; an Studiensequenzanalyse [TSA-] angepasstes KI 0,38-1,99 bzw. RR 0,90; TSA-angepasstes KI 0,49-1,67). Keine statistisch signifikanten Auswirkungen auf die schwerwiegenden $\mathrm{Ne}$ benwirkungen (mit Ausnahme der Mortalität) wurden berichtet (RR 1,02; TSAangepasstes KI 0,7-1,48). In Abwesenheit eines überzeugenden Nachweises für einen Nutzen sprechen wir eine schwache Empfehlung gegen die Nutzung von Kortikosteroiden zur Behandlung von Patienten mit septischem Schock aus, sofern eine angemessene Flüssigkeits- und Vasopressortherapie in der Lage ist, die hämodynamische Stabilität wiederherzustellen.

In einer Studie war die Beobachtung einer potenziellen Interaktion zwischen der Steroidnutzung und einem ACTHTest nicht statistisch signifikant [312]. Des Weiteren wurde kein Nachweis für diese Differenzierung zwischen auf die Therapie ansprechenden und nicht darauf ansprechenden Patienten in einer aktuellen multizentrischen Studie erbracht [309]. Stichprobenartige Überprüfungen des Kortisolspiegels könnten dennoch bei absoluter Nebenniereninsuffizienz hilfreich sein, es gibt jedoch keine Belege dafür, dass stichprobenartige Überprüfungen des Kortisolspiegels bei Patienten mit septischem Schock, bei denen eine relative Nebenniereninsuffizienz vorliegt (keine angemessene Stressreaktion), hilfreich sind. Kortisolimmunassays könnten den tatsächlichen Kortisolspiegel über- oder unterschätzen, was die Zuordnung der Patienten zu den auf die Therapie ansprechenden und nicht darauf ansprechenden Patienten beeinträchtigt [313]. Obwohl die klinische Signifikanz nicht eindeutig geklärt ist, wird inzwischen anerkannt, dass Etomidat die Hypothalamus-NebennierenAchse hemmt [314, 315]. Des Weiteren hat eine Unteranalyse der CORTICUSStudie ergeben, dass die Nutzung von Etomidat vor der Anwendung von Niedrigdosissteroiden mit einer Erhöhung der 28-Tage-Mortalitätsrate verbunden war [309].

Es ist keine vergleichende Studie zwischen einem Behandlungsschema mit festgelegter Dauer und einem klinisch gesteuerten Behandlungsschema oder zwischen dem Ausschleichen und dem abrupten Absetzen von Steroiden vorhanden. Drei RCT nutzten ein Behandlungsschema mit festgelegter Dauer [307, 309, 312] und in 2 RCT wurde die Dosis verringert, nachdem der Schock abgeklungen war [308, 316]. In 4 Studien wurden die Steroide über einen Zeitraum von mehreren Tagen ausgeschlichen [307-309, 316] und in 2 RCT wurden die Steroide abrupt abgesetzt [312, 317]. Eine Cross-over-Studie zeigte hämodynamische und immunologische Reboundeffekte nach der abrupten Absetzung der Kortikosteroide [318]. Eine Studie wies keinen Unterschied in Bezug auf das Ergebnis bei Patienten mit septischem Schock nach, wenn Niedrigdosishydrokortison für 3-7 Tage genutzt wurde. Daher empfehlen wir das Ausschleichen der Steroide, wenn Vasopressoren nicht mehr benötigt werden [319].

Die Nutzung von Steroiden kann indiziert sein, wenn in der medizinischen Vorgeschichte eine Steroidtherapie oder eine Nebennierendysfunktion vorliegt. $\mathrm{Ob}$ jedoch Niedrigdosissteroide eine präventive Wirkung in Bezug auf die Reduzierung der Inzidenz von Sepsis und septischem Schock bei kritisch erkrankten Patienten haben, kann nicht beantwortet werden. Eine aktuelle große multizentrische Studie wies keine Reduzierung der Entwicklung von septischem Schock bei septischen Patienten nach, die Hydrokortison im Vergleich zu einem Placebo erhielten [320]. Daher sollten Steroide nicht bei septischen Patienten genutzt werden, um einem septischen Schock vorzubeugen. Derzeit laufen zusätzliche Studien, die möglicherweise zusätzliche Informationen liefern, die künftig in der klinischen Praxis genutzt werden können.

Diverse randomisierte Studien zur Nutzung von Niedrigdosishydrokortison bei Patienten mit septischem Schock meldeten einen signifikanten Anstieg von Hyperglykämien und Hypernatriämien [312] als Nebenwirkungen. Eine kleine prospektive Studie ergab, dass wiederholte Bolusanwendungen von Hydrokortison zu einem signifikanten Anstieg des Blutzuckers führten. Dieser Spitzenwerteffekt war während einer kontinuierlichen Infusion nicht feststellbar gewesen. Des Weiteren wurde eine beträchtliche interindividuelle Variabilität in Bezug auf diesen Blutzuckerspitzenwert nach der Hydrokortisonbolustherapie festgestellt [321]. Obwohl kein Zusammenhang zwischen einer Hyperglykämie und Hypernatriämie und den gemessenen Ergebnissen beim Patienten nachgewiesen werden konnte, beinhaltet eine gute Praxis Strategien zur Vermeidung und/oder Detektion dieser Nebenwirkungen.

\section{Blutprodukte}

\section{I.1 | Empfehlung | 2018}

\section{- Wir empfehlen, dass Transfusionen von Erythrozytenkonzentraten bei septischen Patienten nur dann durch- geführt werden, wenn die Hämoglo- binkonzentration bei Erwachsenen auf einen Wert von $<7,0 \mathrm{~g} / \mathrm{dl}$ sinkt und keine Hinweise auf eine anämi- sche Hypoxie, wie z. B. Tachykardie, Hypotension, EKG-Ischämie, Laktata- cidose, eingeschränkte Kompensati- onsmöglichkeiten wie z. B. koronare Herzerkrankung, Herzinsuffizienz oder eine zerebrovaskuläre Insuffi- zienz oder eine akute Hämorrhagie, vorliegen. \\ - SSC-Leitlinienadaptation; Abwei- chung des Evidenzgrads \\ - Konsensstärke: $100 \%$ \\ - Empfehlungsgrad: stark \\ - Evidenzgrad: moderat}

Begründung. Nur eine große randomisierte kontrollierte multizentrische Stu- 
die evaluierte unterschiedliche Transfusionstrigger bei septischen Patienten. Diese TRISS-Studie („transfusion requirements in septic shock trial") untersuchte eine Transfusionsschwelle von $7 \mathrm{~g} / \mathrm{dl}$ gegenüber $9 \mathrm{~g} / \mathrm{dl}$ bei Patienten mit septischem Schock nach der Aufnahme auf der ITS [322]. Die Ergebnisse zeigten eine ähnliche 90-Tage-Mortalität, ischämische Ereignisse und Nutzung von lebenserhaltenden Maßnahmen in beiden Behandlungsgruppen bei einer geringeren Anzahl von Transfusionen in der Gruppe mit der niedrigeren Transfusionsschwelle. Bei Nachuntersuchungen ein Jahr nach Intensivaufenthalt fanden sich keine signifikanten Unterschiede zwischen beiden Gruppen bezüglich Mortalität und Lebensqualität [323]. In einer Post-hocAnalyse der Patienten des TRISS-Trials zeigte sich auch bei Patienten mit schweren Begleiterkrankungen, wie z. B. schwerer Lungenerkrankung oder malignen Erkrankungen, kein Outcomeunterschied zwischen beiden Transfusionstriggern. In der Post-hoc-Analyse fanden sich auch keine Outcomeunterschiede bei Patienten mit elektiver vs. Notfalloperationen und bei Patienten, die auch nach den neuen Sepsis-3-Definitionen einen septischen Schock aufwiesen.

In einer Metaanalyse, die neben der TRISS-Studie noch 12 Kohortenstudien in die Auswertung einschloss, fanden sich auch keine Outcomeunterschiede zwischen einer restriktiven und einer liberalen Transfusionsstrategie bei septischen Patienten [324].

Eine einzelne Observationsstudie mit insgesamt 6016 septischen Patienten fand sogar bei fehlender Mortalitätsreduktion eine vermehrte Häufung von nosokomialen Infektionen und schweren Hypoxämien bei einer liberaleren Transfusionsstrategie während der Intensivtherapie [325].

In 3 großen RCT, die die Effektivität einer Early-goal-Therapie bei septischen Patienten untersuchten, waren Erythrozytentransfusionen mit unterschiedlichen Hämatokritwerten ein Bestandteil einer umfassenderen Sepsismanagementstrategie [326]. Weder in den einzelnen Studien noch in einer Metaanalyse fand sich ein Hinweis auf eine Mortalitätsreduktion durch das
Maßnahmenbündel, bei dem u.a. die Erythrozytentransfusion mit enthalten waren, um eine Hämatokritkonzentration von über $30 \%$ zu erreichen.

Die Querschnittsleitlinien der Bundesärztekammer (BÄK) zur Therapie mit Blutkomponenten und Plasmaderivaten haben eine besondere rechtliche Stellung, $\mathrm{da}$ in den Richtlinien zur Therapie mit Blutkomponenten und Plasmaderivaten (Hämotherapie) nach $₫ 18$ TFG auf die vorliegenden Querschnittsleitlinien verwiesen wird [327]. Auch bei septischen Patienten sollten die Empfehlungen der deutschen Querschnittsleitlinie beachtet werden.

\section{I.2 | Empfehlung | 2018}

\section{- Wir empfehlen, dass von der Ver- wendung von Erythropoietin zur Behandlung einer Anämie bei septi- schen Patienten abgesehen wird. \\ - SSC-Leitlinienadaptation \\ - Konsensstärke: $100 \%$ \\ - Empfehlungsgrad: stark \\ - Evidenzgrad: moderat}

Begründung. Es gibt bislang keine klinische Studie zur Nutzung von Erythropoietin speziell bei septischen Patienten. Die klinischen Studien zur Erythropoietinverabreichung an dem größeren Kollektiv an kritisch erkrankten Patienten zeigten einen kleinen Rückgang des Bedarfs für Erythrozytentransfusionen, ohne dass dies Auswirkung auf die Mortalität hatte [328, 329]. Der Effekt von Erythropoietin bei Sepsis und septischem Schock wird mit hoher Wahrscheinlichkeit nicht vorteilhafter sein als bei anderen kritischen Erkrankungen. Die Verabreichung von Erythropoietin könnte aber mit einer erhöhten Inzidenz von thrombotischen Ereignissen bei kritisch erkrankten Patienten verbunden sein. Unabhängig von einer vorliegenden Sepsis oder einem septischen Schock können aber ggf. Grunderkrankungen des septischen Patienten vorliegen, bei denen eine Indikation zur Verwendung von Erythropoietin vorliegt.

\section{I.3 | Empfehlung | 2018}

- Wir schlagen vor, dass Plasmapräparate zur Korrektur von Gerinnungsanomalien bei septischen Patienten nicht genutzt werden, es sei denn es liegen aktive Blutungen vor oder es sind invasive Eingriffe geplant.

- SSC-Leitlinienmodifikation

- Konsensstärke: $100 \%$

- Empfehlungsgrad: schwach

- Evidenzgrad: sehr niedrig

Begründung. In Deutschland stehen 4 verschiedene Plasmapräparationen zur klinischen Anwendung zur Verfügung: gefrorenes Frischplasma (GFP), das Solvent-Detergent(SD)-behandelte Plasma (SDP), das Methylenblau-Licht-behandelte Plasma (MLP) sowie das lyophilisierte Humanplasma (LHP). Es finden sich in der medizinischen Literatur keine randomisierten klinischen Studien, die unterschiedliche Plasmasubstitutionsregime bzw. die Anwendung der unterschiedlichen Plasmapräparationen bei kritisch kranken Patienten untersucht haben. Aktuelle Empfehlungen basieren hauptsächlich auf Expertenmeinungen, die besagen, dass Plasmapräparationen gegeben werden können, wenn eine dokumentierte Störung der plasmatischen Gerinnung vorhanden ist (Koagulopathie mit Quickwerten $<50 \%$ oder APTT $>45 \mathrm{~s}$ und/oder Fibrinogenspiegel $<1 \mathrm{~g} / \mathrm{l}$ ) und eine aktive Blutung vorliegt bzw. wenn invasive Prozeduren oder Operationen geplant werden. Plasma sollte bei Vorliegen dieser Voraussetzungen in einer Dosierung von 15-20 ml/kg Körpergewicht rasch transfundiert werden [327].

\section{I.4 | Empfehlung | 2018}

- Wir schlagen eine prophylaktische Thrombozytentransfusion bei fehlenden weiteren Blutungsrisiken erst bei Thrombozytenzahlen $<10.000 / \mu \mathrm{l}$ vor. In Abhängigkeit von geplanten Operationen oder anderen invasiven Maßnahmen, dem Vorliegen einer aktiven Blutung oder der Einnahme von Medikamenten, die die Thrombozytenfunktion beeinträchtigen, kann 
eine Thrombozytentransfusion aber auch schon bei Thrombozytenzahlen von $20.000 / \mu$ l bis zu $100.000 / \mu l$ erforderlich sein.

- SSC-Leitlinienmodifikation

- Konsensstärke: $100 \%$

- Empfehlungsgrad: schwach

- Evidenzgrad: sehr niedrig

Begründung. Es sind keine RCT zu prophylaktischen Thrombozytentransfusionen bei septischen oder kritisch erkrankten Patienten bekannt. Spezifische Empfehlungen zur prophylaktischen oder therapeutischen Gabe von Thrombozytenkonzentraten bei septischen Patienten können deswegen nicht ausgesprochen werden. Wir empfehlen auf der Basis der Querschnittsleitlinien der BÄK zur Therapie mit Blutkomponenten, eine prophylaktische bzw. therapeutische Thrombozytentransfusion bei septischen Patienten durchzuführen [327].

\section{J. Immunglobuline}

\section{J.1 | Empfehlung | 2018}

\section{- Wir schlagen vor, dass von der Ver- wendung von intravenösen Im- munglobulinen bei Patienten mit Sepsis oder septischem Schock abge- sehen wird. \\ - SSC-Leitlinienadaptation \\ - Konsensstärke bei Doppelabstim- mung: $100 \%$ \\ - Enthaltungen: 3 \\ - Empfehlungsgrad: schwach \\ - Evidenzgrad: niedrig}

Begründung. Eine große multizentrische RCT $(n=624$; [330]) bei erwachsenen Patienten erbrachte keinen mortalitätssenkenden Effekt einer IVIgGGabe. Die aktuelle Cochrane-Metaanalyse [331] differenziert zwischen Standardimmunglobulinen (polyklonales IVIgG) und IgM-angereichertem polyklonalem Ig (IVIgGMA). In 10 Studien mit IVIgG (1430 Patienten) betrug die 28-Tage- bis 130-Tage-Sterblichkeit in der IVIgG-Gruppe $29,6 \%$ und in der
Placebogruppe 36,5\% (RR 0,81; $95 \%$ KI 0,70-0,93). Für die 7 Studien mit IVIgGMA (528 Patienten) lag die 28Tage- bis 60 -Tage-Sterblichkeit in der IVIgGMA-Gruppe bei $24,7 \%$ und in der Placebogruppe bei $37,5 \%$ (RR 0,66; 95\%-KI 0,51-0,85). Die Aussagekraft wurde für die IVIgG-Studien als niedrig eingestuft basierend auf dem Biasrisiko und der vorhandenen Heterogenität. Für die IVIgGMA-Studien wurde die Aussagekraft als mittelgradig eingestuft basierend auf dem Biasrisiko. Vergleichbare Ergebnisse fanden sich auch in anderen Metaanalysen.

Diese Ergebnisse sind im Einklang mit denjenigen älterer Metaanalysen [332, 333] anderer Cochrane-Autoren. Eines dieser systematischen Reviews [333] hatte 21 Studien eingeschlossen und eine Mortalitätssenkung durch die Ig-Gabe gezeigt (RR 0,77; 95\%-KI 0,68-0,88). Allerdings war diese Mortalitätssenkung bei der Auswertung nur der hochwertigen Studien (insgesamt 763 Patienten) nicht mehr statistisch signifikant unterschiedlich (RR 1,02; $95 \%$-KI 0,84-1,24). In ähnlicher Weise fanden Laupland et al. [332] eine signifikante Letalitätssenkung durch IVIg (OR 0,66; $95 \%$-KI 0,53-0,83; $p<0,005$ ), diese war aber bei Berücksichtigung nur der qualitativ hochwertigen Studien nicht mehr signifikant (OR 0,96; $95 \%$-KI $0,71-1,3 ; p=0,78)$. Zwei ebenfalls ältere Metaanalysen [334, 335] mit weniger strengen oder fehlenden Qualitätskriterien zur Biaserkennung fanden eine signifikante Mortalitätssenkung. Weiterhin muss festgestellt werden, dass es bei Sepsispatienten keinen IgG-Plasmaspiegel-Grenzwert gibt, unterhalb dessen eine Substitution mit IVIgG die Prognose verbessert [336].

Die meisten IVIg-Studien sind klein und einige haben ein hohes Biasrisiko. Die einzige große Studie - durchgeführt mit IVIgG - zeigte keinen Effekt [330]. Die getrennte Betrachtung der Studien mit IVIgGMA und IVIgG weist eine signifikante Heterogenität auf. Indirektheit und Publikationsbias wurden zwar in Betracht gezogen, aber nicht zur Bewertung dieser Empfehlung herangezogen. Der geringe Evidenzgrad führte zur Klassifizierung als „schwache Empfehlung“. Die statistische Information der Studien mit hoher Qualität unterstützt nicht die Annahme eines günstigen Effekts des polyklonalen IVIg. Die Leitlinienexperten regen die Durchführung großer multizentrischer Studien zur Evaluierung der Wirksamkeit weiterer polyklonaler IVImmunglobulin-Präparate bei Patienten mit Sepsis an.

Einen Schritt in diese empfohlene Richtung ging die Phase-II-CIGMA-Studie [337] mit der Gabe eines neuen, doppelt so hoch angereicherten $\operatorname{IgM}(23 \%)$ Immunglobulinpräparats (Trimodulin, Fa. Biotest, Dreieich, Deutschland) bei 160 Patienten mit beatmungspflichtiger ambulant erworbener Pneumonie (sCAP). Zwar war der primäre Endpunkt - Anzahl der ventilatorfreien Tage innerhalb der ersten 28 Tage - nicht signifikant unterschiedlich; ermutigende Ergebnisse der Post-hoc-Analysen in Subgruppen führten immerhin zur Planung einer entsprechenden Phase-III-Studie.

\section{K. Blutreinigung}

\section{K.1 | Empfehlung | 2018}

\section{- Expertenkonsens \\ - Wir empfehlen, dass von Blutrei- nigungstechniken außerhalb von klinischen Studien abgesehen wird. \\ - SSC-Leitlinienmodifikation \\ - Konsensstärke bei Doppelabstim- mung: $100 \%$}

Begründung. Bei der Blutreinigung kommen diverse Techniken zum Einsatz, wie z.B. die Hochvolumenhämofiltration und Hämoadsorption (oder Hämoperfusion), bei denen Sorbenzien, die entweder Endotoxin oder Zytokine entfernen, in Kontakt mit dem Blut gebracht werden. Weitere Verfahren sind: der Plasmaaustausch oder die Plasmafiltration, über die Plasma vom Vollblut getrennt, entfernt und durch herkömmliche Kochsalzlösung, Albumin oder gefrorenes Frischplasma ersetzt wird, sowie das Hybridsystem: die kombinierte Plasma-Filtration-Adsorption („coupled plasma filtration adsorption“, CPFA), bei der die Plasmafiltration und -adsorpti- 
on über eine Harzkartusche kombiniert wird, die Zytokine entfernt.

Sofern diese Modalitäten der Blutreinigung im Vergleich zur konventionellen Behandlung untersucht wurden, waren die dazu durchgeführten Studien klein und nicht verblindet und verfügten über ein hohes Verzerrungspotenzial. Die Patientenauswahl war uneindeutig und unterschied sich in Abhängigkeit von den verschiedenen Techniken. Bei der Hämoadsorption handelt es sich um die am genauesten untersuchte Technik, insbesondere mit Polymyxin-B-immobilisierten, auf Polystyren basierten Fasern zur Entfernung von Endotoxin aus dem Blut. Eine vor kurzem durchgeführte Metaanalyse zeigte einen vorteilhaften Effekt auf die Gesamtmortalität bei Verwendung dieser Technik [338]. Dieser Effekt beruht jedoch auf einer Reihe von Studien, die in einem einzigen Land (Japan) durchgeführt wurden und zwar hauptsächlich von einer einzigen Forschungsgruppe. Eine aktuelle große RCT, die an Patienten mit Peritonitis im Zusammenhang mit einer Organperforation innerhalb von $12 \mathrm{~h}$ nach einer Notoperation durchgeführt wurde, wies keinen Vorteil einer Polymyxin-B-Hämoperfusion in Bezug auf die Mortalität und das Organversagen im Vergleich zur Standardbehandlung nach [339]. Der Schweregrad der Erkrankung der Studienpatienten war jedoch insgesamt gering, wodurch die Ergebnisse limitiert sind. Aktuell beendet wurde eine multizentrische RCT, die eine stärkere Evidenz im Hinblick auf diese Technik zur Verfügung stellen könnte [340]. Die Auswertung der 449 eingeschlossenen Patienten, die alle einen nachgewiesenen, erhöhten Endotoxinspiegel im Blut hatten, ergab keinerlei Vorteil der Behandlung bezüglich der Sterblichkeit, auch nicht in einer vordefinierten Untergruppe mit besonders schwerem Organversagen [341].

Wenige RCT evaluierten die Plasmafiltration als Monotherapie oder in Kombination mit einer Adsorption zur Zytokinentfernung (CPFA). Eine aktuelle RCT, in der die CPFA mit der Standardbehandlung verglichen wurde, ist wegen mangelnder Machbarkeit abgebrochen worden [342]. Etwa die Hälfte der Patienten, die randomisiert der CPFA zugeordnet wurden, war nicht wirksam behandelt, hauptsächlich wegen eines "clotting" im extrakorporalen Kreislauf, was an der CPFA-Umsetzbarkeit zweifeln lässt.

Schließlich bleiben noch aktuelle Studien zu Zytokinadsorbern (Hersteller: CytoSorbents) zu erwähnen. Hier gab es 2018 und Anfang 2019 die ersten beiden kleinen RCT; vorher bestand die spezifische klinische Literatur ausschließlich aus Einzelfallberichten oder Fallserien. Beide Studien waren nicht gepowert, um einen Outcomevorteil darzustellen. In der deutschen Studie von 2018 war zudem das Ziel einer besseren IL-6Elimination nicht nachweisbar [343]. In der ungarischen Studie von 2019 gibt es Hinweise auf einen rascheren Abfall des Prokalzitoninwerts in der behandelten Gruppe [344].

In Anbetracht all dieser Einschränkungen ist die Evidenz sehr gering, was die Entscheidung gegen oder für Blutreinigungstechniken betrifft. Es werden weitere Studien benötigt, um den klinischen Nutzen von Blutreinigungstechniken zu klären.

\section{Antikoagulanzien}

\section{L.1 | Empfehlung | 2018}

\section{- Wir empfehlen, dass von der Ver- wendung von Antithrombin zur Behandlung von Patienten mit Sepsis und septischem Schock abgesehen wird. \\ - SSC-Leitlinienadaptation \\ - Konsensstärke: $100 \%$ \\ - Empfehlungsgrad: stark \\ - Evidenzgrad: moderat}

Begründung. Antithrombin ist das häufigste gerinnungshemmende Mittel, das im Plasma zirkuliert. Der Rückgang der diesbezüglichen Plasmaaktivität zu Beginn der Sepsis korreliert mit einer disseminierten intravasalen Gerinnung (DIC) und einer schlechten Prognose. Eine Phase-III-Studie zu Hochdosisantithrombin bei Erwachsenen mit Sepsis und septischem Schock sowie systematische Reviews von Antithrombin bei kritisch erkrankten Patienten wiesen keinen vorteilhaften Effekt in Bezug auf die Gesamtmortalität nach. Antithrombin wurde mit einem erhöhten Blutungsrisiko in Zusammenhang gebracht [345, 346]. Obwohl Post-hoc-Untergruppenanalysen von Patienten mit Sepsis im Zusammenhang mit DIC eine bessere Überlebensrate bei Patienten nachwiesen, die Antithrombin erhielten, kann dieser Wirkstoff nicht empfohlen werden, bis nicht weitere klinische Studien durchgeführt wurden.

\section{L.2 | Statement | 2018}

\section{- Wir sprechen keine Empfehlung hinsichtlich der Verwendung von Thrombomodulin oder Heparin zur Behandlung von Patienten mit Sepsis oder septischem Schock aus. \\ - SSC-Leitlinienadaptation \\ - Konsensstärke: $100 \%$}

Begründung. Die meisten RCT zu rekombinantem löslichem Thrombomodulin waren auf Sepsis im Zusammenhang mit DIC ausgerichtet und ein systematisches Review deutete auf einen vorteilhaften Effekt auf die Überlebensrate ohne eine Erhöhung des Blutungsrisikos hin [347, 348]. Eine Phase-III-RCT wird momentan bei sepsisassoziierter DIC durchgeführt. Zwei systematische Reviews deuteten auf einen potenziellen Überlebensvorteil bei der Nutzung von Heparin bei Patienten mit Sepsis hin, ohne dass eine Zunahme von großen Blutungen vorlag [349]. Die Gesamtauswirkung bleibt jedoch ungewiss und Heparin kann nicht empfohlen werden, bevor nicht weitere RCT durchgeführt worden sind.

\section{Invasive Beatmung}

Im folgenden Kapitel fassen wir knapp die S3-Leitlinie „Invasive Beatmung und Einsatz extrakorporaler Verfahren bei akuter respiratorischer Insuffizienz" zusammen. Die in der S3-Leitlinie abgegebenen Empfehlungen zu Patienten mit ARDS umfassen in der Mehrheit Patienten mit Sepsis, septischem Schock und/oder einer Pneumonie und erfül- 
len somit die Kriterien der Sepsis-3. Allerdings ist in diesem Kontext keine Studie bekannt, die sich spezifisch mit septischen Patienten befasst hat.

Im Folgenden werden die aus Sicht der Leitlinienkommission der S3-Leitlinie „Sepsis“ wesentlichen Empfehlungen der S3-Leitlinie „Beatmung“ für Patienten mit Sepsis und septischem Schock mit der entsprechenden Evidenz beschrieben und um einige Empfehlungen der SSCLeitlinie ergänzt. Der Aufbau des vorliegenden Texts ist der SSC-Leitlinie angepasst. Für weitergehende Informationen und insbesondere für die detaillierte $\mathrm{Be}$ wertung der zugrunde liegenden Evidenz verweisen wir auf die Langfassung der S3Leitlinie „Invasive Beatmung und Einsatz extrakorporaler Verfahren bei akuter respiratorischer Insuffizienz“.

\section{M.1 | Empfehlung | 2018}

\section{- Wir empfehlen die Beatmung von Patienten mit ARDS mit einem $\mathrm{V}_{\mathrm{T}}$ $\leq 6 \mathrm{ml} / \mathrm{kg}$ Standardkörpergewicht (KG). (- Tab. 1 und 2, Anhang) \\ - Adaptation der S3-Leitlinie Beat- mung \\ - Konsensstärke: $100 \%$ \\ - Empfehlungsgrad: stark \\ - Evidenzgrad: moderat}

Begründung. Mehrere Metaanalysen [350, 351] von RCT, die eine Beatmung mit kleinem $V_{T}$ oder niedrigem endinspiratorischem Atemwegsdruck (PEI) $<30 \mathrm{~cm} \mathrm{H}_{2} \mathrm{O}$ (die in einem $V_{T}<7 \mathrm{ml} / \mathrm{kg} \mathrm{KG}$ resultierten) gegen eine Beatmung mit einem $\mathrm{V}_{\mathrm{T}}$ von $10-15 \mathrm{ml} / \mathrm{kg} \mathrm{KG}$ mit und ohne Änderung des positiven endexspiratorischen Drucks („positive end-expiratory pressure“, PEEP) verglichen haben, zeigen eine Reduktion der Sterblichkeit bei adulten ( $>16$ Jahre) invasiv beatmeten Patienten mit ARDS [352-354].

Die aktuelle internationale Leitlinie der SSC empfiehlt zur Beatmung bei Patienten mit ARDS, die eine schwerere Sepsis bzw. einen septischen Schock aufweisen, ein $V_{T} \leq 6 \mathrm{ml} / \mathrm{kg}$ Standard-KG (starke Empfehlung, hohe Qualität der Evidenz), um einen $\mathrm{P}_{\mathrm{EI}}<30 \mathrm{~cm} \mathrm{H}_{2} \mathrm{O}$ an- zustreben (starke Empfehlung, moderate Qualität der Evidenz).

Aufgrund dieser nachgewiesenen Reduktion der Sterblichkeit von Patienten mit ARDS durch eine Beatmung mit niedrigen Tidalvolumina bei fehlendem Nachweis von relevantem Schaden vergibt die Leitlinienkommission eine starke Empfehlung für die Beatmung von invasiv beatmeten Patienten mit ARDS mit einem Tidalvolumen $\mathrm{V}_{\mathrm{T}} \leq 6 \mathrm{ml} / \mathrm{kg}$ Standard-KG. Die Anwendung von $\mathrm{V}_{\mathrm{T}}$ $\leq 6 \mathrm{ml} / \mathrm{kg}$ Standard-KG, um einen PEI $<30 \mathrm{~cm} \mathrm{H}_{2} \mathrm{O}$ zu erreichen, kann in unterschiedlichem Ausmaß zur Hyperkapnie und respiratorischer Acidose führen. Die resultierende Hyperkapnie und respiratorische Acidose wurden in den RCT in unterschiedlichem Ausmaß toleriert und therapiert [350]. Hyperkapnie und respiratorische Acidose können bei kritisch kranken Patienten den intrakraniellen Druck erhöhen, eine pulmonale Hypertension und eine myokardiale Depression verstärken sowie den renalen Blutfluss reduzieren [355]. Bei Patienten, bei denen aufgrund der Grunderkrankung eine Hyperkapnie und respiratorische Acidose vermieden werden sollen, ist daher Nutzen und Risiko der Reduktion des $\mathrm{V}_{\mathrm{T}}$ individuell zu bewerten [356].

\section{M.2 | Empfehlung | 2018}

- Empfehlungsgrad: stark

- Evidenzgrad: moderat

- Wir empfehlen die invasive Beatmung von Patienten ohne ARDS mit einem $V_{T}$ von 6-8 $\mathrm{ml} / \mathrm{kg}$ Standard-KG.

- Adaptation der S3-Leitlinie Beatmung

- Konsensstärke: $100 \%$

Begründung. Mehrere Metaanalysen belegen eine Reduktion der Beatmungsdauer bzw. der postoperativen pulmonalen Komplikationen (PPC) durch die Anwendung von kleinen $\mathrm{V}_{\mathrm{T}}(6-8 \mathrm{ml} / \mathrm{kg}$ Standard-KG) bei adulten (>16 Jahre) Patienten ohne ARDS, die entweder intraoperativ oder auf der ITS invasiv beatmet wurden [357-359]. Zudem zeigte eine Metaanalyse eine Dosis-WirkungsBeziehung zwischen dem Auftreten von PPC und der Größe des $V_{T}\left(R^{2}=0,39\right.$;
[359]). Aufgrund des nachgewiesenen Nutzens für den Patienten und fehlender Hinweise auf relevante Risiken erteilt die Leitlinienkommission eine starke Empfehlung für die invasive Beatmung von Patienten ohne ARDS mit einem $\mathrm{V}_{\mathrm{T}}$ von 6-8 ml/kg Standard-KG. Die Anwendung eines $\mathrm{V}_{\mathrm{T}}$ von $6-8 \mathrm{ml} / \mathrm{kg}$ StandardKG kann auch bei Patienten ohne ARDS in unterschiedlichem Ausmaß zur Hyperkapnie und respiratorischer Acidose führen [358]. Hyperkapnie und respiratorische Acidose können bei kritisch kranken Patienten den intrakraniellen Druck erhöhen, eine pulmonale Hypertension und myokardiale Depression verstärken sowie den renalen Blutfluss reduzieren [355]. Bei Patienten, bei denen aufgrund der Grunderkrankung eine Hyperkapnie und respiratorische Acidose vermieden werden sollen, sind daher Nutzen und Risiko der Reduktion des $\mathrm{V}_{\mathrm{T}}$ individuell zu bewerten [356].

\section{M.3 | Empfehlung | 2018}

- Wir empfehlen, bei der invasiven Beatmung von Patienten mit ARDS den endinspiratorischen Atemwegsdruck (PEI) $\leq 30 \mathrm{~cm} \mathrm{H}_{2} \mathrm{O}$ zu halten.

- Adaptation der S3-Leitlinie Beatmung

- Konsensstärke: $100 \%$

- Empfehlungsgrad: stark

- Evidenzgrad: moderat

\section{M.4 | Empfehlung | 2018}

\section{- Wir schlagen vor, bei der invasiven Beatmung von Patienten mit akuter respiratorischer Insuffizienz einen endinspiratorischen Atemwegsdruck (PEI) $\leq 30 \mathrm{~cm} \mathrm{H}_{2} \mathrm{O}$ zu halten.}

- Adaptation der S3-Leitlinie Beatmung

- Konsensstärke: $100 \%$

- Empfehlungsgrad: schwach

- Evidenzgrad: moderat

M.5 | Empfehlung | 2018

- Wir schlagen vor, bei der invasiven Beatmung eine inspiratorische 
Druckdifferenz (",driving pressure“) von $\leq 15 \mathrm{~cm} \mathrm{H}_{2} \mathrm{O}$ anzustreben.

- Adaptation der S3-Leitlinie Beatmung; Abweichung des Evidenzgrads

- Konsensstärke: $100 \%$

- Empfehlungsgrad: schwach

- Evidenzgrad: niedrig

Begründung. In einer qualitativ hochwertigen Cochrane-Metaanalyse [351] wurde der Effekt einer lungenprotektiven Beatmung bei Patienten mit ARDS analysiert. Insgesamt wurden 6 RCT in die Metaanalyse einbezogen, nur bei 3 Studien war in der Kontrollgruppe ein erhöhter endinspiratorischer Druck bei der Beatmung der Patienten notwendig [353, 354, 360-362]. Bei insgesamt 1009 Patienten aus diesen 3 Studien zeigte sich in der Interventionsgruppe (511 Patienten) mit der Reduktion des $P_{E I}$ auf $\leq 30 \mathrm{~cm} \mathrm{H}_{2} \mathrm{O}$ ein relatives Risiko von $0,74(0,63 ; 0,87)$ bezüglich der Sterblichkeit $[353,362]$. Die absolute Reduktion der Sterblichkeit betrug $10,7 \%$. Für die analysierten sekundären Outcomeparameter Beatmungsdauer, Verweildauer auf der ITS und Inzidenz eines Multiorganversagens, aber auch für Lebensqualität und kognitives Outcome ist die Datenlage ungenügend.

Trotz der aktuell noch offenen Frage, ob der inspiratorische Beatmungsdruck Hauptdeterminante oder nur ein Resultat anderer Parameter - wie beispielsweise PEEP, Tidalvolumen und Compliance bei der lungenprotektiven Beatmung sowie der Schwere der Erkrankung - ist, spricht die Leitlinienkommission in Abwägung von Nutzen und Schaden eine starke Empfehlung zur Limitierung des endinspiratorischen Beatmungsdrucks $\leq 30 \mathrm{~cm} \mathrm{H}_{2} \mathrm{O}$ bei Patienten mit ARDS und eine schwache Empfehlung bei Patienten mit akuter respiratorischer Insuffizienz aus, da ein Nutzen für die Mehrzahl der Patienten angenommen werden kann.

Das Konzept der inspiratorische Druckdifferenz („driving pressure“) und dessen Limitierung auf $\leq 15 \mathrm{~cm} \mathrm{H}_{2} \mathrm{O}-$ bisher nur auf retrospektiven Daten [363] bzw. einer großen prospektiven Beobachtungsstudie [364] beruhend - hat die Leitlinienkommission in der Abwägung von Nutzen und Schaden zu einer diesbezüglichen (schwachen) Empfehlung veranlasst. Bei adipösen Patienten oder Patienten mit einem erhöhten intraabdominellen Druck kann gegebenenfalls ein höherer endinspiratorischer Druck bis $\mathrm{zu} 35 \mathrm{~cm} \mathrm{H}_{2} \mathrm{O}$ notwendig werden. Während pathophysiologische Überlegungen dies zu rechtfertigen scheinen, da der transpulmonale Druck unter diesen Umständen reduziert sein kann [365], existieren keine prospektiv-randomisierten Studien, die die Unbedenklichkeit eines solchen Vorgehens belegen.

Für die Beatmung von Patienten unter Extrakorporalverfahren wird aufgrund theoretischer Annahmen eine weitere Reduktion des Plateaudrucks [366] und des „driving pressure“ [367] angestrebt. Prospektiv randomisierte Studien, die hierfür Grenzen untersucht haben, fehlen jedoch bisher.

\section{M.6 | Empfehlung | 2018}

- Wir empfehlen, invasiv beatmete Patienten mit einem PEEP nicht unter $5 \mathrm{~cm} \mathrm{H}_{2} \mathrm{O}$ zu beatmen.

- Adaptation der S3-Leitline Beatmung

- Konsensstärke: $100 \%$

- Empfehlungsgrad: stark

- Evidenzgrad: sehr niedrig

\section{M.7| Empfehlung | 2018}

- Wir empfehlen, Patienten mit ARDS mit einem höheren PEEP zu beatmen.

- Adaptation S3-Leitline Beatmung

- Konsensstärke: $100 \%$

- Empfehlungsgrad: stark

- Evidenzgrad: hoch

\section{M.8| Empfehlung | 2018}

- Wir schlagen vor, für die orientierende Einstellung des PEEP eine der im einleitenden Abschnitt dieses Kapitels in der S3-Leitlinie „Beatmung“ vorgeschlagenen bettseitigen Methoden anzuwenden. Unter Berücksichtigung der einfachen Anwendbarkeit schlagen wir orientierend die Einstellung nach der
ARDS-Network-Tabelle vor. (• Tab. 1 und 2, Anhang)

- Adaptation der S3-Leitline Beatmung

- Konsensstärke: $77 \%$

- Empfehlungsgrad: schwach

- Evidenzgrad: niedrig

Begründung. Patienten ohne ARDS

Pathophysiologische Überlegungen legen nahe, Patienten nicht mit einem PEEP von weniger als $5 \mathrm{~cm} \mathrm{H}_{2} \mathrm{O}$ invasiv zu beatmen. Entsprechend lautende Empfehlungen werden in mehreren Leitlinien im Sinne einer Expertenmeinung ausgesprochen [368-371] und wurden beispielsweise in den Kontrollgruppen der oben erwähnten RCT zur Beatmung mit niedrigen Tidalvolumina auch ausnahmslos angewendet. Die aktualisierte Version der SSC-Leitlinie enthält keine allgemeine Empfehlung zur Anwendung von PEEP [2]. Eine wirklich fundierte Evidenz für diese Empfehlung bestand bisher nicht.

Trotz der bisher fehlenden Nachweise positiver Effekte einer Beatmung mit mindestens $5 \mathrm{~cm} \mathrm{H}_{2} \mathrm{O}$ PEEP beim Patienten ohne ARDS auf kritische Outcomeparameter, wie Überleben und ITSVerweildauer, vergibt die Leitlinienkommission aufgrund der nachgewiesenen positiven Effekte auf Oxygenierung und Lungencompliance und wegen fehlender Hinweise auf relevante Risiken eine starke Empfehlung für die Anwendung von mindestens $5 \mathrm{~cm} \mathrm{H}_{2} \mathrm{O}$ PEEP bei invasiv beatmeten Patienten ohne respiratorisches Versagen auf der ITS.

\section{Patienten mit ARDS}

Die aktualisierte Version der SSCLeitlinie 2016 vergibt eine schwache Empfehlung zur Anwendung höherer statt niedriger PEEP-Level in adulten Patienten mit schwerem ARDS (schwache Empfehlung, moderate Qualität der Evidenz; [2]).

Der Effekt einer invasiven Beatmung mit höherem PEEP (typischerweise $12-15 \mathrm{~cm} \mathrm{H}_{2} \mathrm{O}$ und höher) im Vergleich $\mathrm{zu}$ einer konventionellen PEEP-Einstellung (typischerweise $5-10 \mathrm{~cm} \mathrm{H}_{2} \mathrm{O}$ ) bei Patienten mit einem ARDS wurde anhand von qualitativ hochstehenden systematischen Übersichtsarbeiten/Metaanalysen (SR/MA) untersucht 
[372-377], die auf RCT basieren [353, 375, 378-382].

Die Erkenntnisse aus diesen Studien bzw. Metaanalysen lassen sich wie folgt zusammenfassen:

Die Kombination aus hohem PEEP und niedrigem $\mathrm{V}_{\mathrm{T}}$ (um $6 \mathrm{ml} / \mathrm{kg} \mathrm{KG}$ ) ist gegenüber einer Beatmung mit niedrigem PEEP (und hohem oder niedrigem $\mathrm{V}_{\mathrm{T}}$ ) mehrheitlich mit einer signifikant besseren ITS-, Krankenhaus- und (teilweise) Tag-28-Überlebensrate verbunden [353, 372, 373, 376, 377].

Eine invasive Beatmung mit einem höheren $\mathrm{PEEP}$ verbessert die Oxygenierung $\left(\mathrm{p}_{\mathrm{a}} \mathrm{O}_{2}-\mathrm{F}_{\mathrm{I}} \mathrm{O}_{2}\right.$-Ratio) signifikant an Tag 1 und $3[373,379,380]$ und ist nicht mit einer höheren Rate an Barotraumata verbunden [373-377] bzw. geht in Kombination mit einem niedrigen Tidalvolumen sogar mit einer geringeren Rate an Barotraumata einher $[353,383]$. Allerdings vermag eine invasive Beatmung mit höherem PEEP weder die Aufenthaltsdauer aufder ITS noch die Beatmungsdauer signifikant zu verkürzen bzw. die Anzahl an beatmungsfreien Tagen zu erhöhen [373, 377].

Ob bei invasiver Beatmung die Wahl eines höheren PEEP per se zu pulmonalen Langzeitschäden führt, kann aufgrund der derzeitigen Studienlage nicht beantwortet werden. Ebenfalls sind keine Studien bekannt, die den Einfluss einer Beatmung mit höherem PEEP (per se) auf das Langzeitüberleben und die Lebensqualität untersucht haben.

Patienten mit erhöhten ICP

Es sind derzeit keine kontrollierten Studien bekannt, die bei Patienten mit ARDS und erhöhtem ICP den Effekt eines unterschiedlich hohen PEEP auf Überleben, neurologisches Outcome usw. untersucht haben. Die in Reviews [384] zum Ausdruck gebrachten Expertenmeinungen sprechen sich in solchen Situationen für die Wahl eines höheren PEEP unter kontinuierlicher Kontrolle von ICP und zerebralem Perfusionsdruck (CPP) aus.

Von den verschiedenen Möglichkeiten der Einstellung eines für den individuellen Patienten adäquaten PEEP-Niveaus ist aus Sicht der LL-Gruppe die Anwendung der ARDS-Network-Tabelle die am häufigsten verwendete und zugleich einfachste Methode; sie lässt aber individuelle atemmechanische Begebenheiten unberücksichtigt. Gleichzeitig ist die Qualität der zugrunde liegenden Evidenz für die Einstellung eines individuell adäquaten PEEP sowie konkret für die Auswahl der Methode als gering einzuschätzen.

Daher gibt die Leitlinienkommission hier eine schwache Empfehlung für diese Methode als orientierende Hilfe zur Einstellung eines adäquaten PEEP-Niveaus.

Aufgrund der erwähnten Nebenwirkungen sind bei Patienten mit instabiler Herz-Kreislauf-Funktion Nutzen und Risiko eines höheren PEEP individuell zu bewerten. Ebenso sind bei Patienten mit regional unterschiedlicher Ausprägung des ARDS der Nutzen (Rekrutierung atelektatischer Lungenareale, Verminderung von Scherstress) und das $\mathrm{Ri}$ siko (Überdehnung weniger betroffener Lungenareale mit konsekutiver Totraumventilation) bei der Applikation eines höheren PEEP zu berücksichtigen.

\section{M.9 | Empfehlung | 2018}

\section{- Wir schlagen vor, bei invasiv beat- meten Patienten mit ARDS keine Rekrutierungsmanöver (RM) durch- zuführen. \\ - Zusätzliche DSG-Leitlinienempfeh- lung \\ - Konsensstärke: $100 \%$ \\ - Empfehlungsgrad: schwach \\ - Evidenzgrad: moderat}

\section{M.10 | Empfehlung | 2018}

- Wir empfehlen bei invasiv beatmeten
Patienten mit ARDS keine Durchfüh-
rung von Rekrutierungsmanövern
mit endinspiratorischen Drücken
über $50 \mathrm{~cm} \mathrm{H}_{2} \mathrm{O}$.
- Zusätzliche DSG-Leitlinienempfeh-
lung
- Konsensstärke: $100 \%$
- Empfehlungsgrad: stark
- Evidenzgrad: moderat

Begründung. In der aktualisierten Version der SSC-Leitlinie 2016 wird eine schwache Empfehlung für die Anwendung von Rekrutierungsmanövern (RM) in adulten Patienten mit sepsisinduziertem schwerem ARDS gegeben (schwache Empfehlung, moderate Qualität der Evidenz). Die Autoren beziehen sich auf eine ältere Metaanalyse aus dem Jahr 2008, geben im Hintergrundtext jedoch später einschränkend an, dass ausgewählte Patienten mit schwerer Hypoxämie von Rekrutierungsmanövern in Verbindung mit hohem PEEP profitieren könnten und bezeichnen die Evidenz für einen routinemäßigen Einsatz als gering [2]. Die hier verabschiedete Empfehlung beruht auf der Bewertung einer aktuellen Metaanalyse von 10 prospektiv-randomisierten Studien, die den Einfluss von RM auf die Krankenhaussterblichkeit zusammenfasst [385]. Bei den insgesamt 1594 Patienten mit ARDS führten RM im Vergleich zur Kontrollgruppe zu einer signifikant geringeren Krankenhaussterblichkeit von $36 \%$ in der RM-Gruppe gegen $42 \%$ in der Kontrollgruppe (RR 0,84; $95 \%$-KI 0,74-0,95; $\mathrm{I}^{2}=0 \%$ ). Die Durchführung von RM war dabei nicht mit einer höheren Inzidenz von Barotraumen assoziiert, senkte aber nicht die Notwendigkeit der Durchführung von Rescue-Therapien wie ECMO oder NO-Inhalation. Die meisten eingeschlossenen Studien fanden keine Unterschiede in der Beatmungsdauer sowie in der Dauer des ITS- oder Krankenhausaufenthalts; diese Variablen ließen sich wegen uneinheitlicher Erfassung jedoch nicht systematisch auswerten.

Die RM waren in der Mehrzahl der berücksichtigten Studien der Metaanalyse Teil eines studienspezifischen Maßnahmenbündels zur Optimierung der Beatmung (u.a. wurden die folgenden Maßnahmen mit der Durchführung von RM gepaart: intermittierende Bauchlage, inhalative Gabe von NO, kontinuierliche Muskelrelaxation). Somit ist der Effekt auf die Krankenhaussterblichkeit nur indirekt auf RM zurückzuführen. Zudem wurden in den verschiedenen Studien unterschiedliche Formen von Rekrutierungsmanövern durchgeführt, wodurch die Vergleichbarkeit eingeschränkt ist. Die eingeschlossenen Studien zeigten zum überwiegenden Teil ein hohes Biasrisiko, da z.B. Informationen über Randomisierung in 5 Studien fehlten. Die Submetaanalyse der 3 Studien, die 
ein niedriges Biasrisiko trugen, konnte für die Anwendung von RM keinen Überlebensvorteil zeigen [385].

Im Oktober 2017 verglich eine randomisierte multizentrische Untersuchung bei 1010 Patienten mit moderatem und schwerem ARDS die Anwendung eines Rekrutierungsmanövers mit anschließender PEEP-Einstellung nach der besten Compliance des respiratorischen Systems (Rekrutierungsmanöver-plushoher-PEEP-Gruppe) gegenüber einer maschinellen Beatmung ohne Anwendung eines Rekrutierungsmanövers und der PEEP-Einstellung nach der niedrigen $\mathrm{F}_{\mathrm{I}} \mathrm{O}_{2}$-PEEP-Tabelle des ARDS-Network (Kontrollgruppe; [386]). PEEP Einstellung nach der besten Compliance des respiratorischen Systems resultierte in einem höheren PEEP-Niveau. Im Rahmen des Rekrutierungsmanövers wurde ein PAW $>50 \mathrm{~cm} \mathrm{H}_{2} \mathrm{O}$ angewandt. In der Rekrutierungsmanöver-plus-hohe-PEEPGruppe verstarben 277 der 501 Patienten (55,3\%) und in der Kontrollgruppe 251 der 509 Patienten (49,3\%) bis zum Tag 28 (HR 1,20; 95\%-KI 1,01-1,42; $p=0,041$ ). Verglichen mit der Kontrollgruppe zeigte die Rekrutierungsmanöver-plus- hohePEEP-Gruppe eine erhöhte Sterblichkeit nach 6 -Monaten $(65,3 \%$ vs. $59,9 \%$; HR $1,18$; $95 \%$-KI 1,01-1,38; $p=0,04)$, geringere Anzahl beatmungsfreier Tage (5,3 vs. 6,4; Differenz-1,1; $95 \%$-KI -2,1 bis $-0,1$; $p=0,03)$, und ein erhöhtes Risiko für ein Barotrauma (5,6\% vs. $1,6 \%$; Differenz 4,0\%; $95 \%$-KI 1,5-6,5\%; $p=0,001)$. Die ITS- und Krankenhausverweildauer unterschieden sich nicht. Damit ergab sich für die Anwendung eines Rekrutierungsmanövers mit einem $\mathrm{PAW}>50 \mathrm{~cm} \mathrm{H}_{2} \mathrm{O}$ und anschließender PEEP-Einstellung nach der besten Compliance des respiratorischen Systems bei Patienten mit ARDS eine Übersterblichkeit.

\section{M.11 | Statement | 2018}

- In der S3-Leitlinienkommission „Beatmung" besteht erklärter Dissens darüber, ob bei erwachsenen $\mathrm{Pa}$ tienten nach akutem Abfall des Atemwegsdrucks (z. B. durch Diskonnektion, Bronchoskopie) und konsekutiver akuter Hypoxämie ein RM vorzuschlagen ist.

- Adaptation der S3-Leitline Beatmung

- Konsensstärke: $100 \%$

Begründung. Die Evidenz beschränkt sich auf 2 kleine Studien mit Untersuchungen physiologischer Surrogatparameter im „Cross-over“-Design. Eine Erhöhung des inspiratorischen Drucks während Absaugmanövern konnte einen Abfall des Lungenvolumens weitgehend verhindern [387]. RM nach offenem Absaugen verhinderte einen längerfristigen Abfall der arteriellen Oxygenierung und des Lungenvolumens [388].

Aufgrund der sehr geringen Qualität der Evidenz war es das Ziel der Diskussion in der Leitlinienkommission einen Expertenkonsens zu formulieren. Aufgrund der Heterogenität der Expertenmeinungen innerhalb der Leitlinienkommission erklären die Mitglieder zur Frage der Anwendung von RM nach akutem Atemwegsdruckabfall den einen expliziten Dissens. Es bleibt damit dem klinischen Anwender vorbehalten, eine individuelle Risiko-Nutzen-Abwägung zu dieser Frage durchzuführen.

\section{M.12 | Empfehlung | 2018}

- Wir empfehlen, dass die Bauchlage bei Patienten mit ARDS und Einschränkung der arteriellen Oxygenierung $\left(\mathrm{p}_{\mathrm{a}} \mathrm{O}_{2} / \mathrm{F}_{1} \mathrm{O}_{2}<150\right)$ durchgeführt werden soll.

- SSC-Leitlinienadaptation sowie Adaptation der S3-Leitlinie Beatmung unter Anlehnung an die S2e-Leitlinie Lagerungstherapie und Frühmobilisation zur Prophylaxe oder Therapie von pulmonalen Funktionsstörungen 2015 [389]

- Konsensstärke: $92 \%$

- Empfehlungsgrad: stark

- Evidenzgrad: moderat

M.13 | Empfehlung | 2018

- Wir schlagen vor, dass ein Bauchlagerungsintervall von mindestens $16 \mathrm{~h}$ angestrebt werden soll. Die Bauchlagerung sollte frühzeitig er- wogen und nach Indikationsstellung unverzüglich umgesetzt werden.

- Adaptation der S3-Leitlinie Beatmung unter Anlehnung an die S2eLeitlinie Lagerungstherapie und Frühmobilisation zur Prophylaxe oder Therapie von pulmonalen Funktionsstörungen 2015 [389]

- Konsensstärke: $92 \%$

- Empfehlungsgrad: schwach

- Evidenzgrad: moderat

Begründung. Die Empfehlungen M.12 und M.13 stellen eine Adaptation der S2eLeitlinie zur Lagerungstherapie dar. Die Behandlung des Themas folgt grundsätzlich der S2e-Leitlinie: „Lagerungstherapie und Frühmobilisation zur Prophylaxe oder Therapie von pulmonalen Funktionsstörungen - Revision 2015“ [390], allerdings wurden eine Cochrane-Analyse [391] und eine systematische Übersicht [392], die nach Redaktionsschluss der Quellleitlinie erschienen sind, hier mit aufgenommen. Die Cochrane-Analyse von Bloomfield et al. [391] sieht einen möglichen Überlebensvorteil durch Anwendung der Bauchlage für diejenigen Patientengruppen, die entweder frühzeitig nach Auftreten des Lungenversagens in die Bauchlage gebracht wurden oder einer prolongierten Bauchlagerung unterzogen wurden oder zu Beginn der Maßnahme eine schwere Hypoxämie aufwiesen. $\mathrm{Zu}$ einem vergleichbaren Fazit kommen Park et al. in ihrer Metaanalyse bisher durchgeführter randomisierter Studien.

In einer im Jahr 2013 publizierten multizentrischen Studie im prospektivrandomisierten Design [278] wurden 237 Patienten mit moderatem oder schwerem ARDS frühzeitig $(<48 \mathrm{~h})$ nach Auftreten der Erkrankung in die Bauchlage gebracht (16h oder mehr täglich für ca. 7 Tage), während die Patienten der Kontrollgruppe in Rückenlage behandelt wurden. Alle Patienten wurden lungenprotektiv beatmet und in der Frühphase des ARDS wurde eine Muskelrelaxation mit Cisatracurium vorgenommen. Die 90-Tage-Sterblichkeit betrug 23,6\% in der Bauchlagerungsgruppe und $41 \%$ in der Kontrollgruppe $(p<0,001 ; \mathrm{OR}=0,44)$. Die Inzidenz 
an Komplikationen war nicht unterschiedlich zwischen den Gruppen, aber Patienten der Kontrollgruppe wiesen eine signifikant höhere Inzidenz an kardialen Arrhythmien auf (Qualität der Evidenz 1a). In mehreren Metaanalysen [77, 393] und einer aktuellen CochraneAnalyse [391] wurde gezeigt, dass bei Patienten mit ARDS $\left(\mathrm{p}_{\mathrm{a}} \mathrm{O}_{2} / \mathrm{F}_{\mathrm{I}} \mathrm{O}_{2}<150\right)$ und lungenprotektiver Beatmungsstrategie die frühe Anwendung prolongierter Bauchlagerung $\mathrm{zu}$ einer signifikanten Senkung der Sterblichkeit im Vergleich zur Rückenlagerung führt (Qualität der Evidenz 2b). Bei Patienten mit ARDS und einem $\mathrm{p}_{\mathrm{a}} \mathrm{O}_{2} / \mathrm{F}_{\mathrm{I}} \mathrm{O}_{2}$-Verhältnis $<150$ wies die Nutzung der Bauchlage im Vergleich zur Rückenlage innerhalb der ersten $36 \mathrm{~h}$ nach Intubation, wenn diese über einen Zeitraum von $>16 \mathrm{~h}$ pro Tag aufrechterhalten wurde, eine verbesserte Überlebensrate auf [394, 395].

Folgende Komplikationen wurden während der Bauchlagerung beschrieben: Gesichtsödeme (20-30\%), Druckulzera in den Bereichen Gesicht/Hornhaut, Becken, Knie (ca. 20\%), „Nichttoleranz"während Bauchlagerung (=Husten, Pressen, Beatmungsprobleme ca. 20\%), Herzrhythmusstörungen (ca. 5\%), Mamillennekrosen, Druckulzera der Tibiavorderkante (Einzelberichte), Tubusoder Katheterdislokationen (ca. 1-2\%) und Nervenschäden. Die retrospektive Analyse der multizentrischen randomisierten Studie von Guerin et al. wies eine höhere Inzidenz von Druckstellen und Hautulzera in der Bauchlagerungsgruppe (14,3/1000 Beatmungstage) im Vergleich zur Rückenlage auf (7,7/1000 Beatmungstage, $p=0,002$; [266]).

Die frühzeitig nach Entwicklung/ Diagnose eines schweren ARDS begonnene und ausreichend lange durchgeführte Bauchlagerung ist mit einem hohen zu erwartenden Patientennutzen verknüpft. Das in den Studien und Metaanalysen beschriebene Risikoprofil ist vertretbar und beherrschbar. Voraussetzung hierfür ist die interdisziplinärroutinierte Anwendung mit Kenntnis der Ein- und Ausschlusskriterien, der sicheren praktischen Durchführung sowie der frühzeitigen Identifikation besonderer Risikopatienten und -konstellationen.
Für Patienten mit akuten abdominellen Erkrankungen kann derzeit aufgrund mangelnder Untersuchungen keine Empfehlung bezüglich Art und Dauer einer Bauchlagerung abgegeben werden. Patienten mit abdomineller Adipositas (CT-Definition: sagittaler abdomineller Durchmesser $\geq 26 \mathrm{~cm}$ ) entwickelten während länger dauernder Bauchlagerung (im Mittel 40h) im Vergleich zu Patienten ohne ähnliche Konfiguration signifikant häufiger ein Nierenversagen ( $83 \%$ vs. $35 \%, p<0,01)$ und signifikant häufiger eine hypoxämische Hepatitis (22\% vs. $2 \%, p=0,015$; [396]).

Bauchlagerung kann bei akuten traumatischen oder nichttraumatischen zerebralen Läsionen einen Anstieg des intrakraniellen Drucks und (bei unveränderter Hämodynamik) eine Reduktion des zerebralen Perfusionsdrucks bewirken. Allerdings kann die durch die Bauchlagerung induzierte Verbesserung des pulmonalen Gasaustausches die zerebrale Oxygenierung steigern.

\section{M.14 | Empfehlung | 2018}

\section{- Wir empfehlen nicht die Hochfre- quenzbeatmung bei erwachsenen Patienten mit ARDS. \\ - Adaptation der S3-Leitline Beatmung \\ - Konsensstärke: $100 \%$ \\ - Empfehlungsgrad: stark \\ - Evidenzgrad: hoch}

Begründung. Zwei Metaanalysen zeigen keine Reduktion der Krankenhausbzw. 30-Tage-Sterblichkeit und z.T. eine längere Beatmungs- sowie Intensivbehandlungsdauer durch die Anwendung von „high frequency oscillation ventilation" (HFOV) bei vornehmlich adulten (>18 Jahre) Patienten mit moderatem und schwerem ARDS $\left(\mathrm{p}_{\mathrm{a}} \mathrm{O}_{2} / \mathrm{F}_{\mathrm{I}} \mathrm{O}_{2}<200\right.$; [397, 398]). Eine aktuelle RCT [399] zeigt keine Änderung der 30-Tage-Letalität, während eine andere aktuelle RCT [400] eine Zunahme der Krankenhausletalität mit HFOV beobachtete. Vor diesem Hintergrund wird die Anwendung der HFOV bei Patienten mit ARDS nicht empfohlen (Empfehlungsgrad stark).

\section{M.15 | Statement | 2018}

\section{- Wir können derzeit keine Empfehlung für oder gegen die Ermöglichung der Spontanatmung bei Patienten in der Frühphase $(\leq 48 \mathrm{~h})$ eines schweren ARDS abgeben. \\ - Adaptation der S3-Leitline Beatmung - Konsensstärke: $100 \%$}

Begründung. Obwohl eine RCT [401] eine Reduktion der Hazard Ratio (HR) für die 90-Tage-Letalität, die 28-Tage-Letalität, die Inzidenz eines Pneumothorax und eine Zunahme der Anzahl an beatmungsfreien und organversagensfreien Tage durch die Gabe von hochdosiertem Cisatracurium in den ersten $48 \mathrm{~h}$ zeigte, kann die Blockade der Spontanatmung durch die Gabe von Muskelrelaxanzien in der Frühphase des schweren ARDS nicht generell empfohlen werden. Dies ist begründet durch die methodologischen Mängel, die insbesondere die Teststärke (Power), aber auch Einschlusskriterien, eine nichtleitliniengerechte tiefe Analgosedierung in beiden Behandlungsgruppen, eine hochdosierte fixe Muskelrelaxation mit Cisatracurium ohne Trainof-four-Überwachung und die Gabe von Cisatracurium in der Hälfte der Patienten in der Placebogruppe sowie eine nicht nachvollziehbare überhöhte Inzidenz eines Pneumothorax in der Placebogruppe betreffen [402, 403]. Kritisch muss auch der Hinweis der S3-Leitlinie Analgesie, Sedierung und Delirmanagement in der Intensivmedizin [402] gewertet werden, dass die deutliche Übersedierung die Letalität in beiden Behandlungsgruppen negativ beeinflusst haben könnte [404, 405]. Zentral ist zu betonen, dass in der vorliegenden RCT in beiden Untersuchungsgruppen kein relevanter Spontanatmungsanteil bei den $\mathrm{Pa}$ tienten vorlag und somit die RCT neutral $\mathrm{zu}$ der gestellten Frage ist. Im Gegensatz zur vorliegenden RCT [401] weist eine andere Studie nach kompletter Zwerchfellinaktivität von 18-69h eine ausgeprägte Zwerchfellatrophie mit nachfolgender Zwerchfelldysfunktion und erschwerten Entwöhnung von der Beatmung nach [406]. Andererseits erlauben Studien zur Spontanatmung mit APRV/ 
BIPAP an Patienten mit leichtem und moderatem ARDS $\left(\mathrm{p}_{\mathrm{a}} \mathrm{O}_{2} / \mathrm{F}_{\mathrm{I}} \mathrm{O}_{2} \geq 100\right.$; [407]) oder retrospektive Beobachtungsstudien keine Aussage, dass eine erhaltene Spontanatmung hinsichtlich des VALI unbedenklich bei schwerem ARDS $\left(\mathrm{p}_{\mathrm{a}} \mathrm{O}_{2} / \mathrm{F}_{\mathrm{I}} \mathrm{O}_{2}\right.$ $<100)$ ist. Eine neuere RCT an $138 \mathrm{~Pa}$ tienten mit moderatem und schwerem $\operatorname{ARDS}\left(\mathrm{p}_{\mathrm{a}} \mathrm{O}_{2} / \mathrm{F}_{\mathrm{I}} \mathrm{O}_{2}\right.$ mm Hg $\left.122 \pm 47\right)$ zeigte eine kürzere Beatmungs- und ITS-Verweildauer mit erhaltener Spontanatmung [408]. Zudem war die Sterblichkeitsrate auf der ITS mit früher Spontanatmung zwar nicht signifikant geringer ( $19,7 \%$ vs. $34,3 \% ; p=0,053)$ aber assoziiert mit einer Verbesserung der arteriellen Oxygenierung, der respiratorischen Compliance und einem niedrigeren PEI sowie einem geringeren Bedarf an Analgosedierung $(p<0,05)$.

Daher ist derzeit aus Sicht der LLGruppe unklar, ob die Ermöglichung von Spontanatmung oder eine hochdosierte Gabe von Muskelrelaxanzien in den initialen $48 \mathrm{~h}$ für Patienten mit schwerem ARDS mehr Nutzen oder mehr Risiko bzw. Schaden bedeutet. Somit kann zu dieser Frage aktuell keine Empfehlung abgegeben werden. Bei Patienten, bei denen aufgrund der Grunderkrankung eine Spontanatmung vermieden werden soll (z. B. erhöhter intrakranieller Druck, Rechtsherzversagen etc.) ist daher Nutzen und Risiko der Blockade von Spontanatmung durch Einsatzeiner Muskelrelaxation individuell zu bewerten. Die S3Leitlinie Analgesie, Sedierung und Delirmanagement in der Intensivmedizin [402] empfiehlt die Muskelrelaxation zurückhaltend zu indizieren, auf den kürzest möglichen Zeitraum zu beschränken und mit einem adäquaten Monitoring der Relaxierungstiefe als auch des Sedierungs- und Analgesiegrads zu begleiten. Dazu wird nochmals im Unterkapitel 5.1 Stellung genommen.

\section{M.16| Empfehlung | 2018}

- Wir schlagen vor, frühzeitig (innerhalb der ersten $48 \mathrm{~h}$ nach Intubation) eine unterstützende Beatmung zur Ermöglichung von Spontanatmung mit Ausnahme des schweren ARDS einzusetzen.

- Adaptation der S3-Leitline Beatmung
- Konsensstärke: $100 \%$

- Empfehlungsgrad: moderat

- Evidenzgrad: niedrig

Begründung. In RCT mit Patienten mit mildem und moderatem ARDS [404, 409] als auch bei Patienten mit akutem respiratorischem Versagen [410] wurde mit assistierter Spontanatmung im Vergleich $\mathrm{zu}$ einer kontrollierten Beatmung eine Reduktion des intrapulmonalen RechtsLinks-Shunts, eine Verbesserung der arteriellen Oxygenierung und des HZV, eine Reduktion der Analgosedierung oder eine Reduktion der Beatmungsdrücke bei vergleichbarer Oxygenierung bzw. einer verbesserten $\mathrm{CO}_{2}$-Elimination beobachtet. Diese kleineren RCT und eine internationale Kohortenstudie mit auf einem Propensity-Score basiertem Matchingalgorithmus [411] weisen bei Patienten mit mildem und moderatem ARDS oder akuter respiratorischer Insuffizienz auf keine höhere Rate von beatmungsassoziierten Komplikationen oder höhere ITS- oder Krankenhausletalität infolge einer erhaltenen Spontanatmung hin. In einer neueren RCT an 138 Patienten mit moderatem und schwerem ARDS $\left(\mathrm{p}_{\mathrm{a}} \mathrm{O}_{2} / \mathrm{F}_{\mathrm{I}} \mathrm{O}_{2} \mathrm{mmHg}\right.$ $122 \pm 47)$ wurde eine initial erhaltene Spontanatmung unter APRV/BIPAP mit einer volumenkontrollierten $\mathrm{Be}$ atmung mit einem Tidalvolumen von $6 \mathrm{ml} / \mathrm{kg}$ standardisiertem KG („low $\mathrm{V}_{\mathrm{T}}$ ventilation“, LOVT) verglichen [408]. APRV wurde mit einem hohen Beatmungsdruck $\left(\mathrm{P}_{\text {high }}\right)$ von $<30 \mathrm{~cm} \mathrm{H}_{2} \mathrm{O}$ und einem niedrigen Beatmungsdruck $\left(\mathrm{P}_{\text {low }}\right)$ von $5 \mathrm{~cm} \mathrm{H}_{2} \mathrm{O}$ durchgeführt. Verglichen mit der LTV-Gruppe war in der APRVGruppe die Anzahl der beatmungsfreien Tage höher (19 [,interquartile range“, IQR] $8-22$ vs. 2 [IQR $0-15$ ]; $p<0,001$ ) und die Verweildauer auf ITS kürzer $(p=0,003)$. Die Sterblichkeitsrate auf der ITS betrug in der APRV-Gruppe $19,7 \%$ vs. $34,3 \%$ in der LTV-Gruppe $(p=0,053)$ und war assoziiert mit einer Verbesserung der arteriellen Oxygenierung, der respiratorischen Compliance und einem niedrigeren endinspiratorischen Druck sowie einem geringeren Bedarf an Analgosedierung $(p<0,05)$.
Dennoch liegen keine multizentrischen RCT mit adäquater Patientenzahl vor, die den primären Endpunkt Letalität bzw. die Inzidenz von VALI untersuchten und damit den Vorteil einer frühzeitigen assistierten Beatmung belegen.

Allerdings empfiehlt auch die S3-Leitlinie Analgesie, Sedierung und Delirmanagement in der Intensivmedizin [402] ein protokollbasiertes Sedierungsregime, das spontan atmende, möglichst wache und kooperative Patienten, die die intensivmedizinisch erforderlichen Maßnahmen gut tolerieren (RASS 0/-1), anstrebt. Dies ist nur durch eine frühzeitige unterstützende Beatmung möglich. Eine zu tiefe Analgosedierung kann zu einer erhöhten Sterblichkeit beitragen und soll daher vermieden werden.

Aufgrund der aktuellen Datenlage, die Nachweise für eine Verbesserung wichtiger, aber nicht kritischer Endpunkte (Verbesserung Oxygenierung, Reduktion der Beatmungsdrücke und verminderter Sedierungsbedarf) bei gleichzeitig fehlenden relevanten Risiken liefert, und unter Berücksichtigung der Empfehlungen der S3-Leitlinie Analgesie, Sedierung und Delirmanagement schlägt die Leitlinienkommission daher frühzeitig eine unterstützende Beatmung zur Ermöglichung von Spontanatmung bei respiratorischer Insuffizienz mit Ausnahme des schweren ARDS vor (Empfehlungsgrad: schwach). Bei Patienten, bei denen aufgrund der Grunderkrankung eine Spontanatmungsaktivität vermieden werden soll (z. B. erhöhter intrakranieller Druck, Rechtsherzversagen etc.) ist Nutzen und Risiko einer assistierten Beatmung individuell kritisch $\mathrm{zu}$ bewerten.

\section{M.17| Empfehlung | 2018}

\section{- Wir empfehlen keine intravenöse und inhalative Gabe von $\beta_{2}$-Mimetika zur Prophylaxe oder zur adjunktiven Therapie bei Patienten mit ARDS. \\ - Adaptation der S3-Leitline Beatmung \\ - Konsensstärke: $100 \%$ \\ - Empfehlungsgrad: stark \\ - Evidenzgrad: moderat}

Begründung. Eine Metaanalyse über 3 prospektive randomisierte placebokon- 
trollierte Studien mit den intravenösen oder inhalativen $\beta_{2}$-Mimetika Salbutamol/Albuterol konnte keinen Vorteil für $\beta_{2}$-Mimetika als adjuvante Therapie beim ARDS zeigen (28-Tage-Sterblichkeit RR 1,04 [0,5-2,16 95\%-KI], I² $83 \%, p$ 0,92). Darüber hinaus treten in den mit $\beta_{2}$ Mimetika behandelten Patienten signifikant mehr unerwünschte Ereignisse (Tachykardien und Arrhythmien) auf [412]. Die Qualität der in die Metaanalyse eingegangen Studien wurde aufgrund eines niedrigen Biasrisikos und in Bezug auf die beatmungsfreien Tage und organversagensfreie Tage als hoch eingeschätzt.

Die aktualisierte Version der SSC 2016 enthält eine starke Empfehlung gegen die Anwendung von $\beta_{2}$-Mimetika bei Patienten mit ARDS ohne Bronchospasmus (starke Empfehlung, moderate Qualität der Evidenz; [413]).

Aufgrund des fehlenden Nutzens bei gleichzeitigen deutlichen Hinweisen für einen möglichen Schaden durch die Gabe von $\beta_{2}$-Mimetika bei Patienten mit ARDS und zudem hoher Qualität der zugrunde liegenden Evidenz vergibt die Leitlinienkommission eine starke Empfehlung gegen diese Therapie.

Die Empfehlung bezieht sich nicht auf invasiv beatmete Patienten mit einer akuten durch einen Bronchospasmus verursachten Atemwegsobstruktion. Im Fall einer solchen akuten oder akut-auf-chronischen obstruktiven Störung (Bronchospasmus) bei invasiv beatmeten Patienten besteht die Indikation zur inhalativen Applikationen von $\beta_{2}$-Mimetika (z. B. über Vernebler). Hierzu verweisen wir auf die entsprechenden Behandlungsleitlinien für Patienten mit „chronic obstructive pulmonary disease“ (COPD) bzw. Asthma bronchiale [414], die sich zurzeit in Überarbeitung befinden.

\section{M.18 | Empfehlung | 2018}

- Wir empfehlen bei adulten Patienten, die länger als $24 \mathrm{~h}$ invasiv beatmet wurden, ein Protokoll zur Entwöhnung von der invasiven Beatmung (Weaningprotokoll) anzuwenden, um standardisiert die Bewertung der Entwöhnungsbereitschaft („,readiness to wean") zu evaluieren, die Spontanatmungsversuche (,spontaneous breathing trial") durchzuführen und die Kriterien zur Beendigung der invasiven Beatmung bzw. Extubation/ Dekanülierung zu überprüfen.

- Adaptation der S3-Leitline Beatmung

- Konsensstärke: $100 \%$

- Empfehlungsgrad: stark

- Evidenzgrad: moderat

Begründung. Mehrere Metaanalysen zeigen eine Reduktion der Beatmungs-, der Weaning- und der Intensivbehandlungsdauer durch die Anwendung von standardisierten Weaningprotokollen bei adulten ( $>18$ Jahre) maschinell beatmeten Patienten im Rahmen der Intensivbehandlung [415, 416].

Alle Metaanalysen belegen, dass die Anwendung von standardisierten Weaningprotokollen nicht mit einer Zunahme von Komplikationen oder nachteiligen Auswirkungen, wie z. B. erhöhte Rate an Weaningversagen, Reintubation, Tracheotomie, assoziiert ist [415].

Mehrere Metaanalysen zeigen bei neurologischen bzw. neurochirurgischen Patienten keine Reduktion der Beatmungs-, der Weaning- und der Intensivbehandlungsdauer durch die Anwendung von standardisierten Weaningprotokollen [415, 415-417]. Für den Einsatz von Weaningprotokollen bei dieser Patientengruppe kann somit aktuell kein Vorteil, aber auch kein Nachteil gesehen werden.

Für Patienten im prolongierten Weaning (erfolgreiches Weaning nach $>3$ SBT bzw. >7-tägiger Beatmungsdauer) findet sich eine unzureichende Studienlage, weshalb keine Empfehlung für den Einsatz von Weaningprotokollen abgegeben werden kann [418].

\section{M.19| Statement | 2018}

- Wir sprechen keine Empfehlung
hinsichtlich der Verwendung einer
nichtinvasiven Beatmung bei Pati-
enten mit sepsisinduziertem ARDS
aus.
- SSC-Leitlinienadaptation
- Konsensstärke: $100 \%$

Begründung. Die NIV könnte Vorteile bei sepsisinduziertem Lungenversagen haben wie z. B. bessere Kommunikationsfähigkeit, reduzierter Sedierungsbedarf und die Vermeidung einer Intubation. Eine NIV könnte jedoch die Nutzung eines niedrigen Atemzugvolumens oder das Erreichen von angemessenen PEEP-Werten ausschließen, beides Beatmungsstrategien, für die ein Nutzen sogar bei leichtem bis schwerem ARDS nachgewiesen wurde [419, 420]. im Gegensatz zu Indikationen wie dem kardiogenen Lungenödem oder einer Exazerbation einer chronisch-obstruktiven Lungenerkrankung (COPD), wo die NIVNutzung nur kurzzeitig erfolgt, dauert es beim ARDS oft Tage oder Wochen, bis eine Besserung eintritt, und eine länger andauernde NIV-Nutzung kann zu Komplikationen führen wie z. B. zu einer Schädigung der Gesichtshaut, einer ungenügenden Nahrungszufuhr und einer mangelhaften Erholung der Atemmuskulatur.

Einige kleine RCT haben einen Vorteil bei der NIV-Nutzung bei leichtem ARDS oder ARDS im Frühstadium oder hypoxischem De-novo-Lungenversagen nachgewiesen; diese Studien wurden jedoch an stark selektierten Patientenpopulationen durchgeführt [421, 422]. In jüngerer Zeit verglich eine größere RCT an Patienten mit hypoxämischem Lungenversagen die NIV mit der traditionellen Sauerstofftherapie oder der High-flow-Nasenkanüle [423]. Diese Studie wies eine verbesserte 90-Tage-Überlebensrate mit Highflow-Sauerstoff im Vergleich zur Standardtherapie oder NIV auf; die NIVTechnik war jedoch nicht standardisiert und die Erfahrung der Studienzentren variierte. Obwohl High-flow-Sauerstoff hier nicht adressiert wird, ist es möglich, dass diese Technik künftig eine größere Rolle bei der Behandlung von hypoxischem Lungenversagen und ARDS spielen wird.

Angesichts der Ungewissheit, ob die Kliniker ARDS-Patienten identifizieren können, bei denen sich eine NIV vorteilhaft auswirken könnte, haben wir keine Empfehlung für oder gegen diese Maßnahme ausgesprochen. Falls die NIV-Methode bei Patienten mit ARDS genutzt 
wird, empfehlen wir eine engmaschige Überwachung des Atemzugvolumens.

\section{M.20 | Empfehlung | 2018 \\ - Wir empfehlen die Anwendung von protokollbasierten Spontanat- mungsversuchen bei mechanisch beatmeten Patienten mit Sepsis. \\ - SSC-Leitlinienadaptation \\ - Konsensstärke: $100 \%$ \\ - Empfehlungsgrad: stark \\ - Evidenzgrad: hoch}

Begründung. Tägliche spontane Atmungsversuche bei sorgfältig ausgewählten Patienten reduzieren die Dauer der mechanischen Beatmung und die Entwöhnungsdauer sowohl in individuellen Studien als auch in der gepoolten Analyse der individuellen Studien [415, 424, 425]. Diese Atmungsversuche sollten in Verbindung mit einem spontanen Aufwachversuch durchgeführt werden [426]. Die erfolgreiche Absolvierung von spontanen Atmungsversuchen führt zu einer hohen Wahrscheinlichkeit eines erfolgreichen frühzeitigen Absetzens der mechanischen Beatmung bei nachweislich minimaler Schädlichkeit.

\section{N. Sedierung und Analgesie}

\section{N.1 | Empfehlung | 2018}

\section{- Expertenkonsens \\ - Wir empfehlen die Minimierung einer kontinuierlichen oder diskontinu- ierlichen Sedierung bei beatmeten Sepsispatienten bis zu festgelegten Titrationsendpunkten. \\ - Der Zielwert auf der Richmond Agi- tation Sedation Scale (RASS) für alle intensivmedizinisch behandelten $\mathrm{Pa}$ - tienten ist außerhalb von speziellen Indikationen 0/-1. \\ - SSC-Leitlinienadaptation; zusätzliche DSG-Ergänzung \\ - Konsensstärke: $100 \%$}

Begründung. Die Eingrenzung der Nutzung einer Sedierung bei kritisch erkrankten beatmeten Patienten reduziert die Dauer der mechanischen Beatmung und die Aufenthaltsdauer auf der ITS und im Krankenhaus und ermöglicht eine frühere Mobilisierung [427, 428]. Während diese Daten aus Studien stammen, die an einem großen Spektrum an kritisch erkrankten Patienten durchgeführt wurden, besteht wenig Grund zur Annahme, dass bei septischen Patienten nicht die gleichen Vorteile nachweisbar sind.

Diverse Strategien haben nachweislich die Nutzung von Sedativa und damit die Dauer der mechanischen Beatmung reduzieren können. Durch das Pflegepersonal gesteuerte Protokolle, die eine Sedierungsskala beinhalten, führen mit großer Wahrscheinlichkeit zu besseren Ergebnissen; allerdings hängt der Nutzen von den vorhandenen lokalen Gewohnheiten und der Praxis ab $[429,430]$. Eine andere Option für die systematische Begrenzung des Einsatzes von Sedierungsmaßnahmen ist die bevorzugte Verabreichung von intermittierend anstelle von kontinuierlich verabreichten Sedativa [431, 432]. Eine tägliche Sedationsunterbrechung („daily sedation interruption“, DSI) war mit verbesserten Ergebnissen in einer monozentrischen randomisierten Studie im Vergleich zur Standardbehandlung verbunden [425]. Allerdings wurde in einer multizentrischen RCT kein Vorteil im Zusammenhang mit einer DSI festgestellt, wenn die Patienten unter Nutzung eines Sedierungsprotokolls behandelt wurden. Zudem war dieses mit erhöhtem Aufwand für das das Pflegepersonal verbunden [433]. Eine vor Kurzem durchgeführte Cochrane-Metaanalyse ergab keine starke Evidenz dafür, dass eine DSI die Dauer der mechanischen Beatmung, die Mortalität, die Aufenthaltsdauer auf der ITS oder im Krankenhaus sowie die Nebenwirkungsraten oder den Medikamentenverbrauch von kritisch erkrankten Patienten reduziert. Allerdings ist die Interpretation dieser Ergebnisse durch eine hohe klinische Heterogenität limitiert [434].

Eine andere Strategie besteht in der primären, alleinigen Nutzung von Opioiden und der Vermeidung von Sedativa, wobei in einer monozentrischen Studie nachgewiesen wurde, dass diese
Strategie bei der Mehrheit der beatmeten Patienten realisierbar und mit einer schnelleren Entwöhnung von der mechanischen Beatmung verbunden war [435]. Weiterhin kann die Nutzung von schnell wirkenden Medikamenten, wie z.B. Propofol und Dexmedetomidin, zu besseren Ergebnissen führen als die Verwendung von Benzodiazepinen [436-438]. Die aktuellen Leitlinien zu Schmerzen, Unruhe und Delirium enthalten weitere Angaben zur Umsetzung des Sedierungsmanagements einschließlich nichtpharmakologischer Ansätze für das Management von Schmerzen, Unruhe und Delirium [439].

Unabhängig von der Herangehensweise steht ein großer Umfang an Evidenz zur Verfügung, die den Vorteil einer Begrenzung der Sedierung bei Patienten demonstriert, die eine mechanische Beatmung benötigen und bei denen keine Kontraindikationen vorliegen. Dementsprechend sollte dies die Best Practice für alle kritisch erkrankten Patienten sein einschließlich der Patienten mit Sepsis.

Im Vergleich zur SSC-Leitlinie, die als Einzelempfehlung vorschlägt, eine „kontinuierliche oder diskontinuierliche Sedierung zu minimieren und Sedierungsprotokolle mit spezifischen Sedierungszielen anzustreben", empfiehlt die deutsche Leitlinie bei intensivmedizinisch behandelten Patienten mit höchstem Empfehlungsgrad außerhalb spezifischer Indikationen (z.B. therapeutische Sedierung im Rahmen eines erhöhten intrakraniellen Drucks, Bauchlagerungstherapie im Rahmen des ARDS) einen RASSZielwert von $0 /-1$.

Hinsichtlich der angewandten Methodologie zur Festlegung von Evidenzstärken unterscheidet sich die DAS-Leitlinie von der der SSC. In der deutschen Leitlinie kam das "Oxford level of evidence“ (Oxford LoE) zum Einsatz. In der SSCLeitlinie handelt es sich um ein nicht abgestuftes Best-Practice-Statement.

Zur Diagnostik und Behandlung von Agitation, Schmerzen und Delir sowie zum Einsatz von Sedativa wurde im Jahre 2015 die aktualisierte S3-Leitlinie „Analgesie, Sedierung und Delirmanagement in der Intensivmedizin (DAS-Leitlinie 2015)“ (AWMF-Nr. 001/012, gültig bis 30.08.2020) veröffentlicht [440]. Sie ist 
unter der Federführung der Deutschen Gesellschaft für Anästhesiologie und Intensivmedizin (DGAI) und der Deutschen Interdisziplinären Vereinigung für Intensiv- und Notfallmedizin (DIVI) sowie unter Beteiligung von weiteren 15 medizinischen Fachgesellschaften entwickelt und verabschiedet worden. Die Empfehlungen dieser nationalen Leitlinie gelten für Deutschland.

\section{O. Blutzuckerkontrolle}

\section{1 | Empfehlung | 2018 \\ - Wir empfehlen einen protokollierten Ansatz für das Blutzuckermanage- ment bei ITS-Patienten mit Sepsis, bei denen die Insulindosisgabe be- ginnt, wenn 2 aufeinanderfolgende Messungen einen Blutzuckerspiegel von $>180 \mathrm{mg} / \mathrm{dl}$ ergeben. Bei diesem Ansatz sollte bevorzugt ein oberer Blutzuckerspiegel von $\leq 180 \mathrm{mg} / \mathrm{dl}$ statt eines oberen Zielblutzucker- spiegels von $\leq 110 \mathrm{mg} / \mathrm{dl}$ angestrebt werden. \\ - SSC-Leitlinienadaptation \\ - Konsensstärke: $100 \%$ \\ - Enthaltung: 1 \\ - Empfehlungsgrad: stark \\ - Evidenzqualität: hoch}

\section{2 | Empfehlung | 2018}

\section{- Expertenkonsens \\ - Wir empfehlen, dass die Blutzucker- werte alle 1-2 h kontrolliert werden, bis die Blutzuckerwerte und die Insu- lininfusionsraten stabil sind. Danach sollte alle $4 \mathrm{~h}$ eine Kontrolle bei Pati- enten erfolgen, die Insulininfusionen erhalten. \\ - SSC-Leitlinienadaptation \\ - Konsensstärke: $100 \%$}

Begründung. Eine große monozentrische RCT aus dem Jahr 2001 demonstrierte eine Reduzierung der ITSMortalität bei einer intensivierten intravenösen Insulinbehandlung (LeuvenProtokoll) mit einem Blutzuckerzielwert von $80-110 \mathrm{mg} / \mathrm{dl}$ [441]. Eine zweite ran- domisierte Studie zu einer intensivierten Insulintherapie unter Verwendung des Leuven-Protokolls umfasste internistischen ITS-Patienten auf 3 internistischen ITS mit einer erwarteten Aufenthaltsdauer auf der ITS von mehr als 3 Tagen. In dieser Studie lag keine Reduzierung der Gesamtmortalität vor [442]. Seit diese 2 Studien veröffentlicht wurden, sind diverse RCT [443-450] und Metaanalysen [451-457] zur intensivierten Insulintherapie durchgeführt worden. Die RCT untersuchten gemischte Populationen von chirurgischen und internistischen ITS-Patienten und wiesen nach, dass eine intensivierte Insulintherapie $\mathrm{zu}$ keinem signifikanten Rückgang der Mortalität führte, die NICE-SUGAR-Studie erbrachte sogar einen Nachweis für einen Anstieg der Sterblichkeit [446]. Alle Studien zeigten eine viel höhere Inzidenz einer schweren Hypoglykämie (Glukose $\leq 40 \mathrm{mg} / \mathrm{dl}$; 6-29\%) bei der Durchführung einer intensivierten Insulintherapie auf. Diverse Metaanalysen bestätigten, dass eine intensivierte Insulintherapie bei chirurgischen, medizinischen oder gemischten ITS-Patientenpopulationen nicht mit einem Mortalitätsvorteil verbunden ist. Die Metaanalyse von Song et al. [457] evaluierte ausschließlich septische Patienten und ergab, dass eine intensivierte Insulintherapie nicht $\mathrm{zu}$ einer Veränderung der 28-Tage- oder 90-Tage-Letalität führt und mit einer höheren Inzidenz von Hypoglykämien verbunden ist. Schwellenwert für die Initiierung eines Insulinprotokolls waren in der NICE-SUGAR-Studie Blutzuckerwerte $>180 \mathrm{mg} / \mathrm{dl}$ mit einem oberen Blutzuckerzielwert von $<180 \mathrm{mg} / \mathrm{dl}$. Die NICE-SUGAR-Studie ist die größte und bisher überzeugendste Studie zur Blutzuckerkontrolle bei Intensivpatienten, da in diese zahlreiche ITS und Krankenhäuser sowie eine sehr heterogene $\mathrm{Pa}$ tientenpopulation einbezogen wurden. Diverse medizinische Organisationen, einschließlich der American Association of Clinical Endocrinologists, der American Diabetes Association, der American Heart Association, dem American College of Physicians und der Society of Critical Care Medicine haben Konsenserklärungen zur glykämischen Kontrolle von Krankenhauspatienten veröffentlicht
[458, 459]. Diese Erklärungen beinhalten im Allgemeinen avisierte Blutzuckerzielwerte zwischen 140 und $180 \mathrm{mg} / \mathrm{dl}$. Da keine Evidenz vorliegt, die belegen würde, dass sich Zielwerte zwischen 140 und $180 \mathrm{mg} / \mathrm{dl}$ von Zielwerten zwischen 110 und $140 \mathrm{mg} / \mathrm{dl}$ unterscheiden, wird in den vorliegenden Empfehlungen ein oberer Blutzuckerzielwert von $\leq 180 \mathrm{mg} / \mathrm{dl}$ genutzt, ohne einen unteren Zielwert festzulegen, mit Ausnahme der Hypoglykämie. Engere Wertebereiche, wie z. B. $110-140 \mathrm{mg} / \mathrm{dl}$, können bei ausgewählten Patienten angemessen sein, wenn diese Zielwerte ohne signifikante Hypoglykämie erreicht werden können [458, 460]. Bei der Behandlung sollten Hyperglykämien (>180 mg/dl), Hypoglykämien und große Schwankungen der Blutzuckerwerte vermieden werden, da diese mit einer höheren Mortalität in Zusammenhang gebracht wurden [461-466]. Die Fortsetzung von Insulininfusionen, insbesondere beim Absetzen der Ernährungsmaßnahmen, wurde als Risikofaktor für eine Hypoglykämie identifiziert [449]. Eine ausgewogene Ernährung könnte mit einem reduzierten Risiko für eine Hypoglykämie verbunden sein [467]. Eine Hyperglykämie und Glukosevariabilität scheint nicht im Zusammenhang mit erhöhten Mortalitätsraten bei Diabetespatienten im Vergleich zu Nichtdiabetespatienten zu stehen [468-470]. Patienten mit Diabetes, chronischer Hyperglykämie und Niereninsuffizienz im Endstadium könnten höhere Blutzuckerwertebereiche benötigen [471, 472].

\section{3 | Empfehlung | 2018}

- Wir schlagen die bevorzugte Verwendung von arteriellem Blut gegenüber kapillarem Blut für die patientenna-

hen Testverfahren unter Verwendung von Blutzuckermessgeräten vor, falls die Patienten über arterielle Katheter verfügen sollten.

- SSC-Leitlinienadaptation

- Konsensstärke: $90 \%$

- Empfehlungsgrad: schwach

- Evidenzqualität: niedrig 
Begründung. Diverse Faktoren können die Genauigkeit und Reproduzierbarkeit der Point-of-Care-Testverfahren des kapillaren Blutzuckers beeinflussen, zu denen der Typ und das Modell des verwendeten Geräts, die Nutzererfahrung und Patientenfaktoren gehören einschließlich Hämatokrit (falsche Erhöhung bei Anämie), $\mathrm{p}_{\mathrm{a}} \mathrm{O}_{2}$ und Medikamente [473]. Die Plasmablutzuckerwerte, die durch kapillare Blutzuckertestverfahren ermittelt wurden, waren nachweislich potenziell ungenau mit häufigen fehlerhaft gemessenen Erhöhungen [474-476] über alle Blutzuckerwerte hinweg insbesondere jedoch in den hypoglykämischen und hyperglykämischen Wertebereichen [477] und bei Schockpatienten (die Vasopressoren erhalten; [473]). Ein Review von Studien ergab, dass die Genauigkeit von Blutzuckermessungen mittels Blutgasanalysegeräten und Blutzuckermessgeräten, die arterielles Blut nutzen, signifikant höher ist als bei Blutzuckermessgeräten, die kapillares Blut nutzen [475]. Die US-amerikanische Food and Drug Administration (FDA) hat erklärt, dass „kritisch erkrankte Patienten nicht mit einem Blutzucker-Point-of-CareMessgerät getestet werden sollten, weil die Ergebnisse ungenau sein könnten", und die Zentren für Medicare- und Medicaid-Leistungen verfügen über Pläne zur Durchsetzung eines Verbots der Off-Label-Verwendung von Point-ofCare-Blutzuckertestgeräten bei kritisch kranken Patienten [478]. Diverse medizinische Experten haben den Bedarf für ein Moratorium zu diesem Plan gefordert.

Ein Review von 12 veröffentlichten Insulininfusionsprotokollen für kritisch erkrankte Patienten demonstrierte eine große Variabilität in Bezug auf die Dosisempfehlungen und eine hohe Variabilität der Blutzuckerkontrolle [479]. Dieser Mangel an Konsens in Bezug auf die optimale Dosierung von intravenösem Insulin könnte die Variabilität bei Patientenfaktoren reflektieren (Schweregrad der Erkrankung, chirurgische vs. internistische Patienten) oder die Praxismuster (z.B. Ansätze in Bezug auf die Ernährung, intravenöse Dextrose) in dem jeweiligen Setting, in dem diese Protokolle entwickelt und getestet wurden.
Diverse Studien haben darauf hingewiesen, dass computerbasierte Algorithmen $\mathrm{zu}$ einer engmaschigeren glykämischen Kontrolle bei gleichzeitiger Reduzierung des Risikos einer Hypoglykämie führen [480, 481]. Computergestützte Systeme zur Unterstützung der Entscheidungsfindung und vollautomatische Closedloop-Systeme zur Blutzuckerkontrolle sind realisierbar, aber noch nicht Bestandteil der Standardbehandlung. Es besteht ein Bedarf an Studien zu validierten, sicheren und wirksamen Protokollen und Closed-loop-Systemen zur Kontrolle des Blutzuckerspiegels unter Berücksichtigung der Variabilität innerhalb der Sepsispopulation.

\section{P. Nierenersatztherapie}

\section{P.1 | Empfehlung | 2018}

\section{- Wir schlagen vor, dass bei Patienten mit Sepsis und akutem Nierenversa- gen entweder kontinuierliche oder intermittierende Nierenersatzthera- pien angewendet werden. \\ - SSC-Leitlinienadaptation \\ - Konsensstärke: $100 \%$ \\ - Empfehlungsgrad: schwach \\ - Evidenzgrad: moderat}

Begründung. Zahlreiche nichtrandomisierte Studien zeigen eine nichtsignifikante Tendenz in Richtung einer Verbesserung der Überlebensrate unter Nutzung kontinuierlicher Methoden auf [482-488]. Hingegen konnten 2 Metaanalysen [489, 490] in Bezug auf die Krankenhausmortalität keine signifikante Differenz zwischen dem Outcome unter kontinuierlicher Nierenersatztherapie und intermittierender Nierenersatztherapie zeigen. Diese Abwesenheit eines offensichtlichen Vorteils einer Methode gegenüber der anderen ist auch festzustellen, wenn die Analyse auf RCT begrenzt wird [490]. Vier von 5 prospektiven RCT [491-495] stellten keinen signifikanten Unterschied in Bezug auf die Mortalität fest [491, 492, 494, 495]. Eine RCT zeigte eine signifikant höhere Mortalität in der Patientengruppe mit der kontinuierlichen Behandlung
[493]. In dieser Gruppe hatte allerdings eine unausgeglichene Randomisierung zur Folge, dass ein höherer Schweregrad der Erkrankung zur Baseline vorlag. Bei Anwendung eines multivariablen Modells zur Anpassung in Bezug auf den Schweregrad der Erkrankung konnte keine offensichtliche Differenz im Hinblick auf die Mortalität zwischen den Gruppen festgestellt werden. Die meisten Studien, in denen die Nierenersatztherapiemodi bei kritisch erkrankten Patienten verglichen wurden, umfassten eine kleine Patientenzahl und verfügten über ein hohes Verzerrungspotenzial (z. B. Randomisierungsversagen, Modifikationen des therapeutischen Protokolls während des Studienzeitraums, Kombination von verschiedenen kontinuierlichen Nierenersatztherapietypen, heterogene Patientengruppen). Die aktuellste und größte RCT [495] umfasste 360 Patienten und wies keinen signifikanten Unterschied in Bezug auf die Überlebensrate zwischen der kontinuierlichen und intermittierenden Gruppe nach.

\section{P.2 | Empfehlung | 2018}

\section{- Wir schlagen vor, dass kontinu- ierliche Therapien zum besseren Management des Flüssigkeitshaus- halts bei hämodynamisch instabilen septischen Patienten verwendet werden. \\ - SSC-Leitlinienadaptation \\ - Konsensstärke: $100 \%$ \\ - Empfehlungsgrad: schwach \\ - Evidenzgrad: sehr niedrig}

Begründung. Die Evidenz für diese Empfehlung ist weiterhin niedrig. Zwei prospektive Studien [491] haben über eine bessere hämodynamische Toleranz bei einer kontinuierlichen Behandlung berichtet, ohne eine Verbesserung der regionalen Perfusion [496] und ohne einen Überlebensvorteil [491]. Vier andere Studien wiesen keinen signifikanten Unterschied in Bezug auf den MAP oder einen Abfall des systolischen Drucks zwischen den beiden Methoden nach [492, 494, $495,497]$. Zwei Studien fanden eine signifikante Verbesserung in Bezug auf das Erreichen der Zielwerte mit kontinuier- 
lichen Methoden [491-493] hinsichtlich des Flüssigkeitsmanagements.

Zwei weitere RCT zur Wirksamkeit einer kontinuierlichen Nierenersatztherapie wurden kürzlich publiziert. Beide Studien beinhalteten Patienten mit Sepsis und akutem Nierenversagen und zeigten keinen Unterschied in Bezug auf die Mortalität im Zusammenhang mit einer höheren Nierenersatztherapiedosis auf. Zwei große, multizentrische randomisierte Studien zum Vergleich der Dosis einer Nierenersatztherapie (Acute Renal Failure Trial Network in den USA und die RENAL-Studie in Australien und Neuseeland) konnten ebenfalls keinen Vorteil einer aggressiveren Dosierung der Nierenersatztherapie nachweisen [498, 499]. Eine Metaanalyse der Sepsispatienten, die in alle relevanten RCT einbezogen wurden $(n=1505)$, demonstrierte keinen signifikanten Zusammenhang zwischen der Dosis und der Mortalität, wobei jedoch die Punktschätzung vorteilhafter für kontinuierliche Nierenersatztherapiedosen $>30 \mathrm{ml} / \mathrm{kg} \mathrm{KG}$ und Stunde ausfiel. Wegen des Verzerrungspotenzials, der Inkonsistenz und der Ungenauigkeit ist das Vertrauen in die Schätzung sehr gering. Daher sollten weitere Studien zu diesem Aspekt durchgeführt werden. Eine typische Dosis für eine kontinuierliche Nierenersatztherapie wäre $20-25 \mathrm{ml} / \mathrm{kg} \mathrm{KG}$ und Stunde.

\section{P.3 | Empfehlung | 2018}

- Wir schlagen vor, dass von einer Verwendung von Nierenersatzverfahren bei Patienten mit Sepsis und akutem Nierenversagen wegen einer Erhöhung des Kreatinins oder einer Oligurie abgesehen wird, ohne dass Indikationen für ein Nierenersatzverfahren vorliegen (z. B. therapierefraktäre Überwässerung, Acidose, Hyperkaliämie oder Urämiesymptome).

- SSC-Leitlinienadaptation

- Konsensstärke: $100 \%$

- Empfehlungsgrad: schwach

- Evidenzgrad: niedrig

Eine kleine Studie aus dem Jahr 2002 [500] evaluierte das frühzeitige im Vergleich zum „späten“ oder „verzögerten“
Ansetzen der Nierenersatztherapie, wobei diese Studie nur 4 Patienten mit Sepsis umfasste und keinerlei Vorteile einer frühzeitigen kontinuierliche Nierenersatztherapie nachweisen konnte.

2016 sind 2 relevante RCT veröffentlicht worden: Die Ergebnisse suggerieren entweder die Möglichkeit eines Vorteils [501] oder die eines Nachteils [502] im Zusammenhang mit der Mortalität und deuten auf einen erhöhten Einsatz von Dialyse sowie von Katheterinfektionen bei einer frühzeitigen Nierenersatztherapie hin. Die Indikationskriterien und der Zeitpunkt des Beginns der Nierenersatztherapie unterschieden sich bei den beiden Studien. Die Ergebnisse wurden als Ergebnisse niedriger Evidenzsicherheit beurteilt basierend auf der Indirektheit (viele nichtseptische Patienten) und der Ungenauigkeit im Hinblick auf die Mortalität. Die Möglichkeit einer Schädigung (z.B. Katheterinfektionen) verschiebt die Abwägung des Risikos und des Nutzens in Richtung eines späteren Beginns einer Nierenersatztherapie. Gleichzeitig scheinen die unerwünschten Effekte und Kosten die erwünschten Folgen aufzuwiegen. Daher schlagen wir vor, dass von einer Verwendung einer Nierenersatztherapie bei Patienten mit Sepsis und akuten Nierenschäden nur wegen einer Erhöhung des Kreatinins oder einer Oligurie abgesehen wird, ohne dass sonstige definitive Indikationen für eine Dialyse vorliegen.

\section{Q. Bikarbonattherapie}

\section{Q.1 | Empfehlung | 2018}

- Wir schlagen vor, dass von einer Verwendung einer Natriumbikarbonattherapie zum Zwecke einer Verbesserung der Hämodynamik oder zur Verringerung des Vasopressorbedarfs bei Patienten mit hypoperfusionsinduzierter Laktatacidose und einem $\mathrm{pH}$-Wert von $\geq 7,15$ abgesehen wird

- SSC-Leitlinienadaptation

- Konsensstärke: $100 \%$

- Empfehlungsgrad: schwach

- Evidenzqualität: moderat
Begründung. Obwohl eine Natriumbikarbonattherapie hilfreich bei der Begrenzung des Atemzugvolumens bei ARDS bei permissiver Hyperkapnie sein kann, steht keine Evidenz zur Verfügung, die die Nutzung einer Natriumbikarbonattherapie zur Behandlung einer hypoperfusionsinduzierten Laktatacidämie bei Sepsis rechtfertigen würde. Zwei verblindete Cross-over-RCT, die äquimolares Kochsalz mit Natriumbikarbonat bei Patienten mit Laktatacidose verglichen, wiesen keine Unterschiede in Bezug auf die hämodynamischen Variablen oder den Vasopressorbedarf nach [503, 504]. Die Anzahl der Patienten mit einem $\mathrm{pH}$-Wert $<7,15$ in diesen Studien war allerdings gering. Außerdem lag bei den Patienten nicht nur ein septischer Schock vor, sondern sie hatten auch andere Erkrankungen wie z.B. eine Mesenterialischämie. Die Bikarbonatverabreichung war mit einem Natrium- und Volumenanstieg und einem Anstieg des Laktats und $\mathrm{p}_{\mathrm{a}} \mathrm{Co}_{2}$ sowie einem Rückgang des ionisierten Serumkalziums verbunden. Die Auswirkung der Verabreichung von Natriumbikarbonat auf die Hämodynamik und den Vasopressorbedarf bei einem niedrigeren $\mathrm{pH}$-Wert ist nicht bekannt, das gilt auch für die Auswirkung bei anderen pH-Werten.

\section{R. Prophylaxe einer venösen Thromboembolie}

\section{R.1 | Empfehlung | 2018}

\section{- Wir empfehlen eine pharmakolo- gische Prophylaxe einer venösen Thromboembolie (VTE) mittels un- fraktionierten Heparins (UFH) oder niedermolekularem Heparins (NMH), sofern keine Kontraindikationen in Bezug auf die Verwendung dieser Wirkstoffe vorliegen. \\ - SSC-Leitlinienadaptation \\ - Konsensstärke: $100 \%$ \\ - Empfehlungsgrad: stark \\ - Evidenzgrad: moderat}

Begründung. Intensivpflichtige Patienten weisen ein erhöhtes Risiko sowohl 
für die Entwicklung einer tiefen Venenthrombose (TVT) als auch einer Lungenarterienembolie (LAE) auf. Die Inzidenz einer auf der ITS erworbenen TVT beträgt bis zu $10 \%$ [505], während die Inzidenz einer LAE in einem Bereich von $2-4 \%$ liegt $[506,507]$. Gerade Patienten mit Sepsis bzw. septischem Schock weisen ein erhöhtes Risiko für die Entwicklung einer venösen Thromboembolie (VTE) auf, da z.B. die Notwendigkeit zur Applikation von Vasopressoren einen unabhängigen Risikofaktor für eine auf der ITS erworbene TVT darstellt. Im Rahmen einer Metaanalyse konnte dementsprechend gezeigt werden, dass eine pharmakologische VTEProphylaxe mittels unfraktionierten $\mathrm{He}$ parins (UFH) oder niedermolekularen Heparins (NMH) bei kritisch erkrankten Patienten zu einer signifikanten Reduktion des Risikos für die Entwicklung einer TVT sowie einer LAE führt, ohne dass es zu einer signifikanten Zunahme von Blutungskomplikationen kommt. Die Mortalität bei Patienten, die eine medikamentöse VTE-Prophylaxe mittels UFH oder NMH erhielten, war zwar geringer, vermochte allerdings keine statistische Signifikanz zu erreichen [507]. Alle Studien, die in diese Metaanalyse mit einbezogen worden sind, wurden bereits in den SSC-Leitlinien aus dem Jahr 2012 berücksichtigt, im Rahmen derer eine Empfehlung zur Nutzung einer pharmakologischen Prophylaxe ausgesprochen wurde. In der Zwischenzeit wurden keine weiteren prospektiven randomisierten kontrollierten Studien zu dem Themenkomplex der VTE-Prophylaxe bei intensivpflichtigen Patienten publiziert, sodass diese Studien auch die Datenbasis für die im Jahr 2018 publizierten europäischen Leitlinien der European Society of Anaesthesiology (ESA) zur perioperativen VTE-Prophylaxe bei intensivpflichtigen Patienten darstellen [508]. In Bezug auf intensivpflichtige Patienten mit Sepsis bzw. septischem Schock muss allerdings berücksichtigt werden, dass für dieses spezielle Subkollektiv fast ausschließlich indirekte Daten für eine pharmakologische VTE-Prophylaxe vorliegen, da nahezu alle Studien an einer undifferenzierten Population kritisch erkrankter Patienten durchgeführt wurden.
Dementsprechend liegt nur eine einzige große prospektive RCT zur VTE-Prophylaxe (mittels UFH oder NMH vs. Placebo) bei septischen Patienten vor, im deren Rahmen allerdings alle Patienten gleichzeitig noch mit Drotrecogin alfa behandelt wurden [509].

Insgesamt haben wir uns in der hier vorliegenden Leitlinie der SSC-Empfehlung aus dem Jahr 2016 angeschlossen (=SSC-Leitlinienadaptation) und eine starke Empfehlung für eine pharmakologische VTE-Prophylaxe auch bei kritisch kranken Patienten mit Sepsis oder septischem Schock ausgesprochen, da die generelle Effektivität dieser Maßnahme unzweifelhaft ist. Nichtsdestotrotz kann die zugrunde liegende Evidenz für das Subkollektiv intensivpflichtiger Patienten mit Sepsis oder septischem Schock nur als mittelgradig eingestuft werden, da sich die verfügbare Datenbasis durch eine ausgeprägte Indirektheit auszeichnet.

\section{R.2 | Statement | 2018}

\section{- Wir geben keine Empfehlung für die Bevorzugung von NMH gegenüber UFH zur VTE-Prophylaxe ab. \\ - DSG-Leitlinienstellungnahme \\ - Konsensstärke: $100 \%$}

Begründung. Obwohl die generelle Wirksamkeit einer medikamentösen VTE-Prophylaxe unstrittig ist, bleibt dennoch die Frage nach dem zu verwendenden Antikoagulans (NMH, UFH etc.) kontrovers.

Eine Reihe von Studien hat die Nutzung von NMH im Vergleich zu UFH zur VTE-Prophylaxe bei kritisch erkrankten Patienten verglichen. In die Metaanalyse von Alhazzani et al. aus dem Jahr 2013 wurden insgesamt 4 Studien einbezogen [507], die diesen direkten Vergleich von UFH zu NMH zum Ziel hatten. Im Rahmen dieser Metaanalyse wurde deutlich, dass die Verwendung von NHM zur VTE-Prophylaxe mit einem signifikant geringeren Risiko für die Entwicklung einer LAE bzw. einer symptomatischen LAE assoziiert ist, wenngleich das Risiko für die Entstehung einer TVT, einer symptomatischen TVT, von rele- vanten Blutungskomplikationen oder des Versterbens nicht signifikant beeinflusst wurde. Eine weitere Metaanalyse zu dem Thema NMH vs. UFH zur VTE-Prophylaxe bei intensivpflichtigen Patienten aus dem Jahr 2015 konnte unter Einschluss von insgesamt 8 RCT zeigen, dass eine VTE-Prophylaxe mittels NMH mit einem signifikant geringeren Risiko für die Entwicklung einer TVT assoziiert ist und in einem klinischen Nettobenefit im Vergleich zur UFH-Applikation resultierte. Allerdings zeigte sich im Rahmen dieser Metaanalyse kein signifikanter Unterschied in Bezug auf die Entwicklung einer LAE, einer Blutung oder des Versterbens [510].

In Analogie zur Datenlage bei der pharmakologischen VTE-Prophylaxe bei intensivpflichtigen Patienten im Allgemeinen liegt zur Beantwortung der Effektivitäts- bzw. Sicherheitsfrage von $\mathrm{NMH}$ vs. UFH vs. Placebo bei dem Subkollektiv intensivpflichtiger Patienten mit Sepsis bzw. septischem Schock lediglich eine große prospektive randomisierte kontrollierte Studie [509] vor. Wie bei R.1 bereits beschrieben wurden im Rahmen dieser Studie allerdings alle Patienten gleichzeitig noch mit Drotrecogin alfa behandelt. Im Vergleich der mit UFH vs. NMH behandelten Subgruppen mit mindestens schwerer Sepsis ergab sich ein minimaler und nichtsignifikanter Überlebensvorteil in Höhe von $2 \%$ für die mit NMH behandelte Subgruppe septischer Patienten. In Bezug auf die Anwendung von NMH bei rein septischen Patientenkollektiven liegt zudem noch eine Metaanalyse randomisiert kontrollierter Studien ausschließlich aus China vor (11 Studien mit insg. 594 Patienten), im deren Rahmen die Effektivität und Sicherheit des Einsatzes von NMH zusätzlich zur Routinebehandlung bei Patienten mit Sepsis im Vergleich zur alleinigen Routinebehandlung evaluiert wurde. Durch die NMH-Applikation ließ sich zwar sowohl die Krankheitsschwere (Surrogatparameter: APACHEII-Score) als auch die 28-Tage-Mortalität reduzieren, allerdings kam es zu einer signifikanten Zunahme von relevanten Blutungskomplikationen.

Um die Frage nach dem optimalen Präparat für die VTE-Prophylaxe bei 
Patienten mit Sepsis bzw. septischem Schock abschließend beantworten $\mathrm{zu}$ können, müssen darüber hinaus nun noch die folgenden weiteren Aspekte berücksichtigt werden.

\section{Entwicklung einer heparininduzierten Thrombopenie (HIT) II. Ein Cochrane-} Review aus dem Jahr 2012 vermochte einen signifikanten Rückgang der Inzidenz von heparininduzierter Thrombozytopenie (HIT) II bei postoperativen Patienten zu verzeichnen, wenn diese $\mathrm{NMH}$ anstatt UFH erhielten [511]. Es muss allerdings berücksichtigt werden, dass in dieses Cochrane-Review nur 2 Studien mit insgesamt 923 Patienten eingeschlossen werden konnten und sich beide Studien keineswegs spezifisch auf intensivpflichtige oder gar septische Patienten fokussierten.

Dosierung und Monitoring. Die Applikation von UFH kann in einer Dosierung von $5000 \mathrm{IE}$ alle 8 bzw. $12 \mathrm{~h}$ oder mit 7500 IE alle $12 \mathrm{~h}$ erfolgen. Bei den direkt vergleichenden Studien zu NMH vs. UFH bei intensivpflichtigen Patienten erfolgte in allen Untersuchungen eine nur 2-mal tägliche UFH-Applikation. Qualitativ hochwertige Studien unter Einschluss von intensivpflichtigen Patienten mit einem direkten Vergleich von dreimal täglich verabreichtem UFH vs. NMH sind gegenwärtig nicht verfügbar. Eine indirekt vergleichende Metaanalyse aus dem Jahr 2011 konnte jedenfalls keinen signifikanten Unterschied in Bezug auf die Wirksamkeit (Endpunkte: TVT, LAE, relevante Blutung, Versterben) zwischen 2-mal und 3-mal täglich verabreichtem UFH bei den in die Analyse eingeschlossenen Patienten nachweisen [491, 512]. Im Gegensatz hierzu suggerierte ein anderes Review mit Metaanalyse, der ebenso eine indirekte Vergleichsmethodik zugrunde lag, eine höhere Wirksamkeit von 3-mal täglich verabreichtem UFH, bei aber gleichzeitig erhöhtem Blutungsrisiko [513].

Applikationsweise (intravenöse vs. subkutane VTE-Prophylaxe). Aufgrund vielfältiger Einflussfaktoren (z. B. Schockzustand mit Katecholaminpflichtigkeit, positive Flüssigkeitsbilanz mit ausge- prägter Ödembildung, schwerwiegende Organdysfunktionen) besteht im Rahmen einer Sepsis bzw. eines septischen Schocks das Risiko für eine nichtvorhersehbare bzw. gestörte Resorption von subkutan appliziertem UFH bzw. NMH. Gerade bei der subkutanen Applikation von geringen oder moderaten UFHDosen (5000-15.000 IE/24h), so wie sie vor allem im Rahmen der klassischen VTE-Prophylaxe erfolgt, muss mit einer reduzierten Bioverfügbarkeit gerechnet werden. In Analogie hierzu konnte in einer randomisierten doppelblinden Studie an insgesamt $115 \mathrm{~Pa}$ tienten mit akuter proximaler TVT gezeigt werden, dass eine kontinuierliche intravenöse Dauerapplikation im Vergleich zu einer intermittierenden subkutanen Bolusapplikation von UFH (jeweils 30.000 IE/24h) signifikant häufiger $\mathrm{zu}$ einer initialen therapeutischen Antikoagulation führt (i.v.: $70,7 \%$ vs. s.c. $36,8 \%)$. Das insuffiziente therapeutische Ansprechen auf die s.c.-Applikation war wiederum mit einer signifikant höheren Rethromboserate assoziiert (i.v. 3/58 Patienten vs. s.c. 11/57 Patienten; $p=0,024$; [514]). Somit scheint also im Fall von UFH die i.v.-Applikation die sicherste Applikationsweise für Intensivpatienten darzustellen. Ebenso kritisch muss aber auch die subkutane Bioverfügbarkeit von $\mathrm{NMH}$ bei intensivpflichtigen Patienten gesehen werden. So konnte im Rahmen einer klinisch prospektiven Studie unter Einschluss von 89 intensivpflichtigen Patienten gezeigt werden, dass die s.c.Applikation von Enoxaparin $40 \mathrm{mg} \mathrm{zu}$ den Zeitpunkten $4 \mathrm{~h}, 12 \mathrm{~h}$ sowie $24 \mathrm{~h}$ nach Applikation nur in 56,5\%, 39,3\% sowie $12,6 \%$ der Fälle zu dem angestrebten Anti-Xa-Spiegel von 0,1-0,3 IE/ml führte [515]. Vergleichbare Ergebnisse zeigten sich auch in einer prospektiven Observationsstudie unter Einschluss von 55 intensivpflichtigen Patienten. Ein suffizienter Anti-Xa-Spiegel in Höhe von 0,1-0,3 IE/ml wurde hier nur bei $87,3 \%$, $32,7 \%$ sowie $0 \%$ der Patienten zu den Zeitpunkten 4, 12 und 24h nach Applikation von $40 \mathrm{mg}$ Enoxaparin erreicht [516]. Als Risikofaktoren für ein unzureichendes Ansprechen auf die s.c.Applikation wurden ein hohes Körpergewicht, eine hohe Erkrankungsschwere bzw. die Existenz eines Multiorganversagens identifiziert. Vergleichbare Ergebnisse sind auch für andere $\mathrm{NMH}$, wie z.B. Certoparin oder Dalteparin, beschrieben [517, 689]. Durch die höherdosierte Applikation von NMH (z.B. Enoxaparin einmal $1 \mathrm{mg} / \mathrm{kg} \mathrm{KG}$ und Tag oder 2-mal $40 \mathrm{mg}$ pro Tag) ist es jedoch möglich, auch bei intensivpflichtigen $\mathrm{Pa}$ tienten Anti-Xa-Spiegel im angestrebten Zielbereich zu erreichen [518].

Renale Dysfunktion. Bei Patienten mit renaler Dysfunktion muss im Fall einer Nutzung von NMH eine mögliche Bioakkumulation mit nachfolgend erhöhtem Blutungsrisiko bedacht werden. Dies trifft in besonderem Maße für intensivpflichtige Patienten zu, da nahezu ein Drittel der Patienten bereits bei Aufnahme auf die ITS eine Kreatininclearance von weniger als $30 \mathrm{ml} / \mathrm{min}$ aufweist [505]. Eine erste Metaanalyse aus dem Jahr 2006 konnte bei intensivpflichtigen Patienten mit einer Kreatininclearance $\leq 30 \mathrm{ml} / \mathrm{min}$ zeigen, dass die Applikation des NMH Enoxaparin in therapeutischer Standarddosierung ohne Anpassung an die Nierenfunktion zu einer relevanten Bioakkumulation mit signifikant erhöhtem Blutungsrisiko führt. Aufgrund einer insuffizienten Datenlage war diese Metaanalyse allerdings nicht in der Lage, eine relevante Bioakkumulation von Enoxaparin in rein prophylaktischer Dosierung nachzuweisen [519]. In Bezug auf das potenzielle Risiko für eine Bioakkumulation von NMH im Rahmen einer derartigen rein prophylaktischen Dosierung liegen vor allem für das NMH Dalteparin (einmal $5000 \mathrm{IE}$ s.c. pro Tage) weiterführende Studien vor. Im Rahmen einer observierenden Kohortenstudie von Rabbat et al. unter Einschluss von insgesamt 19 intensivpflichtigen Patienten mit einer Kreatininclearance von mehr als $30 \mathrm{ml} / \mathrm{min}$ bei Aufnahme auf die ITS kam es jedenfalls $\mathrm{zu}$ keiner relevanten Bioakkumulation von Dalteparin [520]. Ebenso wurde in der sog. DIRECT-Studie [521] keine Bioakkumulation von Dalteparin bei Patienten $(n=138)$ mit einer kalkulierten Kreatininclearance von weniger als $30 \mathrm{ml} / \mathrm{min}$ nachgewiesen, sodass diese Patientengruppe anschließend auch in 
die sog. PROTECT-Studie mit einbezogen wurde [522]. Im Rahmen der PROTECT-Studie wurden schließlich 118 Patienten mit akutem Nierenversagen analysiert, von denen 60 Patienten einer Behandlung mit Dalteparin und 58 Patienten einer Behandlung mit UFH zugeordnet wurden. In dieser speziellen Subgruppe intensivpflichtiger Patienten ergab sich kein Anhalt für ein vermehrtes Auftreten unerwünschter Ereignisse bei Patienten, die mit Dalteparin im Vergleich zu UFH behandelt wurden. Umgekehrt erwies sich in dieser kleinen Subgruppe die Behandlung mit Dalteparin jedoch auch nicht als effektiver im Vergleich zur VTE-Prophylaxe mittels UFH. Inwiefern andere NMH-Präparationen genauso sicher wie Dalteparin bei Patienten mit akutem Nierenversagen zur VTE-Prophylaxe eingesetzt werden können, bleibt jedoch ungewiss, da keine anderen qualitativ hochwertigen Daten $\mathrm{zu}$ dieser Fragestellung verfügbar sind. Nebenbefundlich suggerierte eine im Jahr 2014 publizierte Kosten-NutzenAnalyse von Fowler et al., die die Datenbasis der PROTECT-Studie nutzte [522], dass die Verwendung von $\mathrm{NMH}$ zum Zweck der VTE-Prophylaxe trotz einer Zunahme der unmittelbaren Kosten für das eingesetzte Antikoagulans $\mathrm{zu}$ einem allgemeinen Rückgang der Gesamtbehandlungskosten führt [523].

Vordergründig scheint also die Nutzung von NMH zur VTE-Prophylaxe bei kritisch erkrankten Patienten grundsätzlich einfach, effektiv und zugleich auch kostengünstig zu sein, sodass die SSCLeitlinie 2016 eine starke Empfehlung für die Nutzung von NMH anstelle von UFH aussprach, wann immer dies praktisch möglich ist. Der zugehörige Evidenzgrad wurde jedoch nur als mittelgradig eingestuft, da die zugrunde liegende Datenbasis durch eine ausgeprägte Indirektheit gekennzeichnet ist, wie z.B. 1.) keine rein septischen Patientenkollektive, sondern gemischte Kollektive intensivpflichtiger Patienten; 2.) systematische Vergleiche zwischen $\mathrm{NMH}$ und UFH zur VTE-Prophylaxe jeweils mit nur 2-mal täglich verabreichtem UFH anstatt mit 3-mal täglicher UFH-Verabreichung. Neben der SSC-Leitlinie 2016 wird aber auch durch die aktuell gültige
S3-Leitlinie „Prophylaxe der venösen Thromboembolie (VTE)“ (AWMF-Leitlinien Register-Nr. 003/001) und die aktuellen europäischen Leitlinien zur perioperativen VTE-Prophylaxe bei intensivpflichtigen Patienten der European Society of Anaesthesiology (ESA; [508]) der Einsatz von NMH auf ITS gegenüber UFH bevorzugt. Demgegenüber wird durch die hiesige Leitlinienkommission gerade der Aspekt der nicht vorhersehbaren subkutanen Resorption bei septischem Schock sowie die nicht auszuschließende Bioakkumulation bei Niereninsuffizienz als kritisch erachtet, sodass in der hier vorliegenden S3-Leitlinie keine klare Empfehlung für die Bevorzugung von (subkutan appliziertem) NMH gegenüber UFH zur VTEProphylaxe ausgesprochen wird (=keine SSC-Leitlinienadaptation, DSG-Leitlinienstellungnahme). Dies ist analog zu einer weiteren Empfehlung innerhalb der S3-Leitlinie „Prophylaxe der venösen Thromboembolie (VTE)“ [524], die bei Blutungsneigung, Niereninsuffizienz oder unsicherer Resorption alternativ auch die intravenöse Verabreichung von UFH in niedriger Dosierung („low dose“) legitimiert, wenngleich dies bisher nicht im Rahmen von Studien validiert wurde. Diesbezüglich wird die i.v.-Applikation von UFH in einer Dosierung von 10.000-15.000 IE/24h als angemessen erachtet.

\section{R.3 | Empfehlung | 2018}

\section{- Wir schlagen eine Kombination aus einer pharmakologischen VTE- Prophylaxe und einer mechanischen Prophylaxe vor, wann immer dies möglich ist. \\ - SSC-Leitlinienadaptation \\ - Konsensstärke: $100 \%$ \\ - Empfehlungsgrad: schwach \\ - Evidenzgrad: niedrig}

Begründung. Die Kombination aus pharmakologischer VTE-Prophylaxe und mechanischer VTE-Prophylaxe mittels intermittierender pneumatischer Kompression (IPK) und/oder medizinischen Thromboseprophylaxestrümpfen (MTPS) ist eine Option bei kritisch erkrankten Patienten mit Sepsis oder septischem Schock. Ein Cochrane-Review [525] aus dem Jahr 2008 konnte unter Einschluss von insgesamt 11 Studien an chirurgischen Hochrisikopatienten $(n=7431)$ zeigen, dass eine kombinierte mechanisch(IPK)-medikamentöse VTEProphylaxe im Vergleich zu einer rein pharmakologischen VTE-Prophylaxe die Inzidenz einer TVT signifikant zu reduzieren vermag. Die eingeschlossenen Studien wiesen allerdings keine ausreichende Power auf, um eine Aussage in Bezug auf die Risikoreduktion für die Entwicklung einer LAE durch eine kombinierte mechanisch(IPK)-medikamentöse VTE-Prophylaxe im Vergleich zu einer rein pharmakologischen VTE-Prophylaxe tätigen zu können. Ein im Jahr 2016 publiziertes Update dieses Cochrane-Reviews unter Einschluss von insgesamt 22 Studien konnte zwar durch eine derart kombinierte mechanisch(IPK)-medikamentöse VTEProphylaxe im Vergleich zu einer rein pharmakologischen VTE-Prophylaxe eine signifikante Reduktion der LAEInzidenz nachweisen, allerdings wurde hier nun ein signifikanter Effekt auf die TVT-Inzidenz knapp verfehlt [526]. Die Qualität der Evidenz muss jedoch als gering eingestuft werden, da sich die Daten aus den untersuchten chirurgischen Patientenkollektiven nur indirekt auf intensivpflichtige Patienten im Allgemeinen bzw. septische Patienten im Speziellen übertragen lassen. Dementsprechend enthalten die vom American College of Chest Physicians (ACCP) veröffentlichten Leitlinien keine Empfehlung zur Nutzung einer Kombinationstherapie im Rahmen der VTEProphylaxe bei kritisch erkrankten Patienten, schlagen jedoch die Nutzung einer derartigen Kombinationstherapie bei chirurgischen Hochrisikopatienten vor [527, 528]. Die aktuell gültige S3-Leitlinie „Prophylaxe der venösen Thromboembolie (VTE)“ (AWMF-Leitlinien, Register-Nr. 003/001) empfiehlt für die ITS den Einsatz von physikalischen Maßnahmen (bevorzugt IPK) vor allem dann, wenn Kontraindikationen gegen eine medikamentöse Prophylaxe bestehen. Eine generelle Empfehlung zur kombinierten mechanisch(IPK)-medi- 
kamentösen VTE-Prophylaxe für ITS ist allerdings nicht beinhaltet. Im Gegensatz hierzu wurde von Seiten der SSC im Jahr 2016 eine schwache Empfehlung für eine Kombinationstherapie zur VTE-Prophylaxe bei kritisch erkrankten Patienten mit Sepsis oder septischem Schock ausgesprochen. Dieser Empfehlung schließt sich die Leitlinienkommission der hier vorliegenden S3-Leitlinie auf Basis der zum Zeitpunkt der Konsensuskonferenz im November 2018 publizierten Literatur an (=SSC-Leitlinienadaptation). Die Datenlage hat sich seither nun insofern verändert, als dass im Februar 2019 der sog. PREVENT-Trial publiziert wurde, im dessen Rahmen die Effektivität einer kombinierten mechanisch(IPK)-medikamentösen VTE-Prophylaxe (IPKGruppe: $n=991$ ) im Vergleich zu einer rein pharmakologischen VTE-Prophylaxe (Kontrollgruppe: $n=1012$ ) bei einem gemischten intensivmedizinischen $\mathrm{Pa}$ tientenkollektiv evaluiert wurde [529, 530, 685]. Dieses Patientenkollektiv bestand größtenteils aus internistischen Intensivpatienten (IPK-Gruppe: 79,4\% vs. Kontrollgruppe: $77,0 \%$ ), während chirurgische Patienten in deutlich geringerem $\mathrm{Maß}$ vertreten waren (IPK-Gruppe: $13,6 \%$ vs. Kontrollgruppe: $14,5 \%$ ). Eine Beatmungspflichtigkeit bestand bei knapp zwei Drittel der eingeschlossenen Patienten (IPK-Gruppe: 66,7\% vs. Kontrollgruppe: $65,9 \%$ ), während eine Katecholaminpflichtigkeit nur bei etwa einem Drittel der Patienten vorlag (IPK-Gruppe: 35,5\% vs. Kontrollgruppe: $36,0 \%)$. Die IPK wurde von den behandelten Patienten jeweils gut vertragen, da sich die Anzahl der Patienten mit Hautläsionen oder Ischämien im Bereich der unteren Extremität zwischen der IPKsowie der Kontrollgruppe nicht signifikant voneinander unterschied. Darüber hinaus wurden keine schwerwiegenden, IPK-assoziierten unerwünschten Ereignisse beobachtet. Vordergründig konnte bei diesem gemischten intensivmedizinischen Patientenkollektiv durch die adjunktive IPK-Nutzung nun allerdings keine Reduktion der Inzidenz einer proximalen TVT im Vergleich zu einer alleinigen medikamentösen VTE-Prophylaxe erzielt werden. Darüber hinaus ließ sich auch die VTE-Inzidenz so- wie die Gesamtsterblichkeit nicht durch die adjunktive IPK-Nutzung reduzieren. Als wichtigste Limitation dieser Studie muss allerdings berücksichtigt werden, dass der primäre Endpunkt (=neu aufgetretene proximale TVT im Bereich der unteren Extremität) bei nur 4,2\% der Patienten in der Kontrollgruppe beobachtet werden konnte, was deutlich seltener als erwartet war. Dies bedingt eine kritische Reduktion der Studienpower, sodass ein klinisch relevanter Effekt der adjunktiven IPK-Nutzung trotz des negativen Studienergebnisses nicht sicher ausgeschlossen werden kann. Außerdem können die Studienergebnisse wiederum nur sehr indirekt auf Patienten mit Sepsis bzw. septischem Schock übertragen werden, da es sich um ein gemischtes intensivmedizinisches Patientenkollektiv handelte und keine Subgruppenanalysen von Patienten mit Sepsis bzw. septischem Schock berichtet wurden. Somit muss der Evidenzgrad für dieses Patientenkollektiv weiterhin als niedrig eingestuft werden und es darf angenommen werden, dass auch eine Berücksichtigung des PREVENTTrials im Rahmen der Evidenzbewertung für die hier vorliegende S3-Leitlinie zu keiner anderslautenden Empfehlung geführt hätte.

\section{R.4 | Empfehlung | 2018}

\section{- Empfehlungsgrad: schwach \\ - Evidenzgrad: niedrig \\ - Wir schlagen eine mechanische VTE-Prophylaxe vor, wenn eine pharmakologische VTE-Prophylaxe kontraindiziert ist. \\ - SSC-Leitlinienadaptation \\ - Konsensstärke: $100 \%$}

Begründung. Ein Teil der an Sepsis oder septischem Schock erkrankten Patienten dürfte relative Kontraindikationen für die Durchführung einer pharmakologischen VTE-Prophylaxe aufweisen. Speziell bei diesen Patienten drängt sich die Durchführung einer mechanischen VTE-Prophylaxe mittels IPK und/oder MTPS geradezu auf, wenngleich die wissenschaftliche Evidenz für deren Nutzung bei intensivpflichtigen Patienten durchaus be- grenzt ist. Bislang sind 2 Metaanalysen veröffentlicht worden, in denen Patientengruppen verglichen wurden, die entweder eine mechanische VTE-Prophylaxe oder keinerlei VTE-Prophylaxe erhielten. Hierbei handelte es sich größtenteils um Patienten, die sich einer orthopädischen Operation zu unterziehen hatten [531, 532]. Während sich das Cochrane-Review von Sachdeva et al. auf die Nutzung von MTPS konzentrierte, lag der Schwerpunkt der Arbeit von Pavon et al. auf der Nutzung von IPK. In den Analysen wurde deutlich, dass sowohl die Nutzung von MTPS als auch die Anwendung der IPK wirksamer als der Verzicht auf eine derartige mechanische Prophylaxe zu sein scheint. Bei der Interpretation der erhobenen Daten muss allerdings berücksichtigt werden, dass eine jeweils unterschiedliche Anzahl von Patienten in den vergleichenden Studienarmen eine zusätzliche pharmakologische Prophylaxe erhielt. Die Evidenz muss dementsprechend als indirekt eingestuft werden. Eine weitere Kohortenstudie unter Einschluss von insgesamt 798 intensivpflichtigen $\mathrm{Pa}$ tienten konnte mittels Propensity-ScoreAnalysen zeigen, dass die IPK (im Vergleich zu den MTPS) die einzig wirklich effektive Maßnahme zur mechanischen VTE-Prophylaxe bei kritisch erkrankten Patienten darstellt [533].

Basierend auf diesen Daten wurde von Seiten der SSC eine schwache Empfehlung für die Nutzung einer mechanischen Prophylaxe bei kritisch erkrankten septischen Patienten mit Kontraindikationen für die Verwendung einer pharmakologischen Prophylaxe ausgesprochen, der sich die hier vorliegende S3-Leitlinie anschließt (=SSC-Leitlinienadaptation). Darüber hinaus gibt es zunehmende Hinweise dafür, dass die IPK wirksamer als die alleinige Nutzung von MTPS bei kritisch erkrankten Patienten $\mathrm{zu}$ sein scheint, sodass diese Methode die bevorzugte Modalität zur mechanischen Prophylaxe darstellt. In Analogie hierzu empfiehlt die S3-Leitlinie „Prophylaxe der venösen Thromboembolie (VTE)“ (AWMF-Leitlinien, RegisterNr. 003/001) für ITS die Durchführung von physikalischen Maßnahmen (v.a. IPK) vor allem dann, wenn Kontrain- 
dikationen gegen eine medikamentöse VTE-Prophylaxe bestehen.

\section{S. Stressulkusprophylaxe}

\section{S.1 | Empfehlung | 2018}

- Wir empfehlen, dass Patienten mit Sepsis oder septischem Schock, bei denen Risikofaktoren für gastrointestinale (GI-)Blutungen vorliegen, eine Stressulkusprophylaxe erhalten.

- SSC-Leitlinienadaptation

- Konsensstärke: $100 \%$

- Empfehlungsgrad: stark

- Evidenzgrad: niedrig

\section{S.2 | Empfehlung | 2018}

- Wir schlagen vor, dass entweder Protonenpumpenhemmer oder Histamin- $\mathrm{H}_{2}$-Rezeptor-Antagonisten verwendet werden, wenn eine Indikation für eine Stressulkusprophylaxe vorliegt.

- SSC-Leitlinienadaptation

- Konsensstärke: $100 \%$

- Empfehlungsgrad: schwach

- Evidenzgrad: niedrig

\section{S.3 | Empfehlung | 2018}

\section{- Expertenkonsens \\ - Wir empfehlen, dass Patienten ohne Risikofaktoren für gastrointestinale Blutungen keine Stressulkusprophy- laxe erhalten. \\ - SSC-Leitlinienadaptation \\ - Konsensstärke: $100 \%$}

Begründung. Ein Stressulkus entwickelt sich im Magen-Darm-Trakt von kritisch erkrankten Patienten und kann mit einer signifikanten Morbidität und Mortalität verbunden sein [534]. Der exakte Mechanismus ist nicht vollumfänglich geklärt, man geht jedoch von einer Beeinträchtigung der protektiven Mechanismen gegen die Magensäure, einer Hypoperfusion der Magenschleimhaut, einer erhöhten Säureproduktion und oxidativen Schädigungen des Ver- dauungstrakts aus [535]. Die stärksten klinischen Prädiktoren des gastrointestinalen Blutungsrisikos bei kritisch kranken Patienten sind eine mechanische Beatmung über einen Zeitraum von $>48 \mathrm{~h}$ und eine Koagulopathie [536]. Eine aktuelle internationale Kohortenstudie ergab, dass eine vorbestehende Lebererkrankung, die Notwendigkeit einer Nierenersatztherapie und ein höherer Schweregrad eines Organversagens unabhängige Prädiktoren eines gastrointestinalen Blutungsrisikos sind [537]. Eine multizentrische prospektive Kohortenstudie ergab, dass die Inzidenz von klinisch bedeutsamen gastrointestinalen Blutungen bei 2,6\% (95\%-KI 1,6-3,6\%) bei kritisch kranken Patienten lag. Andere Beobachtungsstudien wiesen jedoch niedrigere Raten von gastrointestinalen Blutungen nach [538-541].

Ein systematisches Review und eine Metaanalyse von 20 RCT untersuchten die Wirksamkeit und Sicherheit der Stressulkusprophylaxe [542]. Die Prophylaxe mit Histamin- $\mathrm{H}_{2}$-RezeptorAntagonisten $\left(\mathrm{H}_{2} \mathrm{RA}\right)$ oder Protonenpumpenhemmern (PPI) reduziert das Risiko von gastrointestinalen Blutungen im Vergleich zu keiner Prophylaxe (RR 0,44; 95\%-KI 0,28-0,68; niedrige Evidenzqualität), führt jedoch zu einem nichtsignifikanten Anstieg des Pneumonierisikos (RR 1,23; 95 \%-KI 0,86-1,78). Eine große retrospektive Kohortenstudie untersuchte den Effekt einer Stressulkusprophylaxe bei Patienten mit Sepsis und ergab, dass kein signifikanter Unterschied in Bezug auf das Risiko einer C.difficile-Infektion im Vergleich zu keiner Prophylaxe besteht [543]. Bei Indikation für eine Stressulkusprophylaxe sollte die Auswahl der eingesetzten Medikamentengruppe in Abhängigkeit von den Patientencharakteristika und der lokalen Inzidenz von C.-difficile-Infektionen und Pneumonien erfolgen.

Obwohl die veröffentlichten RCT nicht ausschließlich septische Patienten beinhalteten, sind Risikofaktoren für gastrointestinale Blutungen häufig bei Patienten mit Sepsis und septischem Schock vorhanden [536]. Daher ist die Nutzung dieser Datenbasis als Informationsgrundlage für unsere Empfehlungen gerechtfertigt. Basierend auf der verfüg- baren Evidenz überwiegt der erwünschte Nutzen einer Stressulkusprophylaxe das unerwünschte Risiko. Daher haben wir eine starke Empfehlung für die Nutzung einer Stressulkusprophylaxe bei Patienten mit den o.g. Risikofaktoren ausgesprochen. Patienten ohne Risikofaktoren weisen keine große Wahrscheinlichkeit für die Entwicklung klinisch bedeutsamer gastrointestinaler Blutungen während ihres Aufenthalts auf der ITS auf. Daher ist eine Stressulkusprophylaxe nur indiziert, wenn Risikofaktoren vorliegen, und die Patienten sollten in regelmäßigen Abständen dahingehend überprüft werden, ob weiterhin ein Bedarf im Hinblick auf die Nutzen-Risiko-Relation für die Prophylaxe besteht.

Während es Unterschiede in der weltweiten Praxis gibt, haben diverse Umfragen ergeben, dass PPI die am häufigsten genutzten Wirkstoffe in Nordamerika, Australien und Europa sind gefolgt von $\mathrm{H}_{2} \mathrm{RA}$ [537, 544-547]. Eine vor Kurzem durchgeführte Metaanalyse von 19 RCT $(n=2177)$ ergab, dass PPI zwar wirksamer als $\mathrm{H}_{2} \mathrm{RA}$ bei der Prävention von klinisch bedeutsamen gastrointestinalen Blutungen waren (RR 0,39; 95\%-KI 0,21-0,71; $p=0,002$; mittlere Evidenzqualität), aber zu einer nichtsignifikanten Zunahme des Risikos von Pneumonien führten (RR 1,17; $95 \%$ KI 0,88-1,56; $p=0,28$; niedrige Evidenzqualität; [547]). Frühere Metaanalysen $[548,549]$ und eine aktuelle methodisch hochwertige sog. Netzwerkmetaanalyse von 57 RCT ( $n=7293$ Patienten; [550]) waren zu einer ähnlichen Schlussfolgerung gelangt.

Keine der RCT berichteten über ein erhöhtes Risiko für das Auftreten einer C.-difficile-Infektion; wobei eine große retrospektive Kohortenstudie einen kleinen Anstieg des Risikos für eine C.difficile-Infektion mit PPI im Vergleich $\mathrm{zu} \mathrm{H}_{2} \mathrm{RA}$ feststellte $(2,2 \%$ vs. 3,8\%; $p<0,001$; sehr niedrige Evidenzqualität). Im Wesentlichen mangelt es bislang an prospektiven Studien, die einen kausalen Zusammenhang zwischen einer medikamentösen Prophylaxe sowohl für PPI als auch $\mathrm{H}_{2} \mathrm{RA}$ und dem Auftreten einer C.-difficile-Infektion zeigen. Außerdem kamen Kosten-Nutzen-Analysen zu un- 
terschiedlichen Schlussfolgerungen [551, 552].

Dementsprechend muss der Nutzen einer Prävention von gastrointestinalen Blutungen (mittlere Evidenzqualität) gegen eine potenzielle Zunahme der infektiösen Komplikationen (sehr niedrige bis niedrige Evidenzqualität) abgewogen werden. Die Auswahl des prophylaktischen Wirkstoffs wird weitgehend von den individuellen Charakteristika des betreffenden Patienten abhängen sowie von der lokalen Prävalenz der gastrointestinalen Blutungen, Pneumonien und C.difficile-Infektionen. Aufgrund der Unsicherheiten sprechen wir keine bevorzugte Empfehlung für einen Wirkstoff gegenüber dem anderen Wirkstoff aus.

Eine multizentrische Pilotstudie und Metaanalyse (5 RCT, $n=604$ Patienten) zeigte keine signifikanten Unterschiede zwischen einer SUP mit Pantoprazol vs. keine SUP in den Endpunkten klinische bedeutsame Blutung, Pneumonie und C.difficile-Infektionen [553]. Auch die Pilotstudie von Selvanderan et al. an 241 kritisch kranken beatmeten und enteral ernährten Patienten kam zu übereinstimmenden Ergebnissen [554].

Die methodisch hochwertig konzipierte multizentrische sog. SUPICURCT zeigte keinen signifikanten Unterschied im primären Endpunkt 90Tage-Mortalität zwischen einer SUP mit Pantoprazol und Placebo in einem heterogenen Kollektiv von 3298 Intensivpatienten, auch wenn die Patienten in der Pantoprazolgruppe signifikant weniger klinisch bedeutsame gastrointestinale Blutungen hatten $(2,5 \%$ vs. $4,2 \%$; relatives Risiko 0,58 [0,40-0,86]; [555]). Auch in der Subgruppe der Patienten mit Schock zeigte sich kein Unterschied im primären Endpunkt, allerdings bleibt der Anteil von Patienten mit septischem Schock unklar. Derzeit laufende weitere multizentrische Studien zum Effekt einer SUP stehen zum jetzigen Zeitpunkt noch aus (ClinicalTrials.gov, Registrierungsnummer NCT02467621; NCT02718261 und ANZICS-CTG-Studiennummer: 1415-01).

\section{T. Ernährung}

Zugrunde gelegt wurden die Empfehlungen der SSC-Leitlinie 2016, die anhand der S3-Leitlinie der Deutschen Gesellschaft für Ernährungsmedizin e.V. „Besonderheiten der Überwachung bei künstlicher Ernährung“ [556] und unter Berücksichtigung der S2k-Leitlinie „Klinische Ernährung in der Intensivmedizin“ der Deutsche Gesellschaft für Ernährungsmedizin e.V. (DGEM; [557]), der ESICM Leitlinie „Frühe enterale Ernährung“ [558] und der Leitlinie der European Society for Clinical Nutrition and Metabolism (ESPEN) „Clinical Nutrition in the intensive care unit" [559] aktualisiert und ggf. modifiziert wurden. Da nur wenige Daten insbesondere aus randomisierten Studien bei ausschließlich septischen Patienten vorliegen, stellen die meisten Empfehlungen einen Expertenkonsens anhand der Daten aus Studien mit nichtseptischen Patienten dar.

\section{T.1 | Empfehlung | 2018}

\section{- Wir empfehlen, bei kritisch erkrank- ten Patienten mit Sepsis oder sep- tischem Schock, die enteral ernährt werden können, bevorzugt den frühzeitigen Beginn einer enteralen Ernährung. \\ - SSC-Leitlinienadaptation \\ - Konsensstärke bei Doppelabstim- mung: $100 \%$ \\ - Empfehlungsgrad: stark \\ - Evidenzgrad: moderat}

Begründung. Die Bereitstellung einer totalen oder supplementierenden parenteralen Ernährung kann sehr rasch auch bei eingeschränkter gastrointestinaler Toleranz das Erreichen eines angestrebten Kalorien- und Proteinziels gewährleisten. Dies stellt theoretisch einen Vorteil gegenüber der reinen enteralen Ernährung dar, insbesondere für die Patienten, die aufgrund einer eingeschränkten gastrointestinalen Toleranz nicht „voll“ enteral ernährt werden können, was in den ersten Tagen der Behandlung auf der ITS relevant sein kann. Die Bereitstellung einer parenteralen Ernährung ist jedoch invasiver und bedeutet zugangsbedingt potenzielle Komplikationen, einschließlich eines erhöhten Infektionsrisikos. Es besteht allgemeiner Konsens, auch aufgrund anzunehmender physiologischer Vorteile der enteralen Zufuhr beim kritisch kranken Patienten den Vorzug zu geben [557-560].

Um die Frage der Überlegenheit einer parenteralen Ernährung bei Patienten mit Sepsis und septischem Schock anzugehen, wurde die Evidenz für Patienten evaluiert, die frühzeitig enteral ernährt werden konnten, im Vergleich zu Patienten, bei denen eine frühzeitige enterale Ernährung nicht realisierbar war. Das erste systematische SSC-Review untersuchte die Auswirkung einer frühzeitigen parenteralen Ernährung allein oder in Kombination mit einer enteralen Ernährung im Vergleich zu einer ausschließlich enteralen Zufuhr auf die Mortalität bei enteral ernährbaren Patienten. Identifiziert wurden insgesamt 10 Studien mit 2888 Intensivpatienten mit verschiedenen Krankheitsbildern, postoperativ, nach Polytrauma einschließlich SchädelHirn-Trauma sowie mit akuter Pankreatitis [561-570]. Hierbei zeigte sich, dass eine frühzeitige parenterale Ernährung weder die Mortalität (RR 0,97; $95 \%$-KI $0,87-1,08 ; n=2745)$ noch das Infektionsrisiko reduzieren würde (RR 1,52; $95 \%$-KI $0,88-2,62 ; n=2526$ ), allerdings war die Aufenthaltsdauer auf der ITS erhöht (RR 0,90; $95 \%$-KI 0,38-1,42; $n=46$; [571]). Die Evidenzqualität wurde als mittel bis sehr niedrig eingestuft. In der größten randomisierten Studie, die sich mit dieser Frage befasste (CALORIES, $n=2400$ ), traten weniger Episoden einer Hypoglykämie oder von Erbrechen in der Gruppe mit einer frühzeitigen parenteralen Ernährung auf. Es wurden jedoch keine Unterschiede in Bezug auf die Mortalität zwischen den Studiengruppen festgestellt [572].

Seit der systematischen SSC-Übersicht sind zur Frage enteral vs. parenteral 4 aktuelle Metaanalysen erschienen. In einer ersten Metaanalyse zum Vergleich einer enteralen mit einer parenteralen Ernährung bei Intensivpatienten wurden 18 RCT mit 3347 Patienten eingeschlossen. Ein Einfluss der enteralen Ernährung auf die Letalität konnte nicht 
aufgezeigt werden [573]. Es fand sich jedoch eine niedrigere Rate an infektiösen Komplikationen (RR 0,64; 95\%KI 0,48-0,87; $p=0,004)$. Dieser Effekt war ausgeprägter in einer Subgruppe, in der die parenteral ernährten Patienten mehr Kalorien erhalten hatten (RR 0,55; $95 \%$-KI 0,37-0,82; $p=0,003$ ). Der Effekt fehlte, wenn die Kalorienzufuhr in beiden Gruppen gleich gewesen war. Auch wurde ein signifikant kürzerer Intensivaufenthalt beobachtet $(-0,8 ; 95 \%$ $\mathrm{KI}-1,23$ bis 0,$37 ; p=0,0003)$. Insgesamt wurden die Vorteile der enteralen Ernährung vor allem durch die niedrigere Kalorienzufuhr und weniger durch den Zufuhrweg selbst erklärt.

In einer weiteren von der Arbeitsgruppe als qualitativ gut bewerteten Metaanalyse wurden 16 randomisierte Studien mit 3325 Intensivpatienten eingeschlossen. Hier wurde eine frühe (innerhalb von $24 \mathrm{~h}$ ) begonnene enterale Standardernährung mit allen anderen Formen einer Ernährung verglichen. Eine allgemeine Senkung der Mortalität konnte bei früher enteraler Ernährung nicht festgestellt werden und fand sich auch nicht im Vergleich mit einer parenteralen Ernährung. In vorher definierten Subgruppen zeigte sich im Vergleich mit einem verzögerten Ernährungsbeginn $(>24 \mathrm{~h})$ eine signifikant niedrigere Mortalität (OR 0,45; $95 \%$-KI $0,21-0,95 ; p=0,038, I=0 \%)$. Insgesamt entwickelten Patienten mit früher enteraler Ernährung weniger Pneumonien (OR 0,75; 95\%-KI 0,60-0,94; $p=0,012$; $\mathrm{I}^{2}=48 \%$; [574]).

In einer aktuelle Cochrane-Metaanalyse wurden 25 Studien mit 8816 Patienten eingeschlossen. 23 Studien waren randomisiert, 2 quasi-randomisiert. Als Ergebnis zur Frage enterale vs. parenterale vs. kombinierte enteral-parenterale Ernährung war die Evidenz bezüglich der Krankenhausmortalität, 10- und 90-Tage-Mortalität, der Zahl an beatmungsfreien Tagen und unerwünschten Ereignissen unzureichend. Jedoch war die 30-Tage-Mortalität niedriger bei kombiniert enteral-parenteraler Ernährung. Bei enteraler Ernährung kam es seltener zu einer Sepsis als bei parenteraler Ernährung. In die vierte Metaanalyse, deren Qualität von der Arbeitsgruppe als gut bewertet wurde, wurden 23 Studien mit 6478 Patienten eingeschlossen. Im Ergebnis zeigte sich kein Unterschied in der Mortalität und dem Auftreten einer Organdysfunktion. Im Vergleich kam es bei enteraler Ernährung zu weniger Bakteriämien (OR 0,59; 95\%-KI $0,43-0,82 ; p=0,001 ; \mathrm{I}^{2}=27 \%$ ). Dies galt vor allem für chirurgische Intensivpatienten (OR 0,36; KI 0,22-0,59; $p<0,0001$; $\left.\mathrm{I}^{2}=0 \%\right)$. Bei enteraler Ernährung war die Krankenhausverweildauer tendenziell kürzer (MD -0,90; $95 \%$-KI $-1,63$ bis $-0,17 ; p=0,21 ; I^{2}=0 \%$, wobei gastrointestinale Probleme signifikant häufiger auftraten (OR 2,00; 95\%-KI 1,76-2,27; $p=0,00001 ; \mathrm{I}^{2}=0 \%$; [575]).

Nicht berücksichtigt war ferner eine RCT, die bei 2410 Intensivpatienten mit Schock keinen Vorteil einer frühen isokalorischen enteralen $(n=1202)$ gegenüber einer parenteralen $(n=1208)$ Ernährung bezüglich einer Senkung der Mortalität oder anderer sekundärer Komplikationen gezeigt hat (NUTRIREA-2; [576]). Am Tag 28 waren 443 (37\%) der 1202 Patienten in der enteralen Gruppe und $422(35 \%)$ der 1208 Patienten in der parenteralen Gruppe verstorben (absoluter Unterschied, 95\%-KI -1,9 bis 5,$8 ; p=0,33$ ). Die kumulative Inzidenz von auf der ITS erworbenen Infektionen war nicht unterschiedlich (enteral: 173 [14\%], parenteral: 194 [16\%]; HR 0,89 [95\%-KI 0,72-1,09]; $p=0,25)$. Verglichen mit der parenteralen Gruppe zeigte sich bei den enteral ernährten Patienten jedoch eine höhere Inzidenz an Erbrechen (406 [34\%] vs. 246 [20\%]; HR 1,89 [1,62-2,20]; $p<0,0001)$, Diarrhö (432 [36\%] vs. 393 [33\%]; $1,20[1,05-1,37]$; $p=0,009$ ), Darmischämien (19 [2\%] vs. $5[<1 \%] ; 3,84[1,43-10,3] ; p=0,007)$, und dem Auftreten einer akuten Kolonpseudoobstruktion (11 [1\%] vs. 3 $[<1 \%] ; 3,7[1,03-13,2] ; p=0,04)$.

Als Kontraindikationen zur frühzeitigen enteralen Ernährung werden in den neuen ESICM-Leitlinien (2017) genannt: unkontrollierter Schock, metabolische Entgleisung mit unkontrollierter Hypoxämie und Acidose, unkontrollierte obere Gastrointestinalblutung, Magenresidualvolumen $>500 \mathrm{ml} / 6 \mathrm{~h}$, mesenteriale Ischämie, Darmobstruktion, Kompartmentsyndrom, High-output-Fistel ohne distalen Zugang zur Ernährung [558]. Eine gastrale Ernährung kann kontinuierlich oder als Bolusgabe erfolgen, die jejunale Zufuhr bedarf der kontinuierlichen Applikation [557].

Zusammenfassend wird bei ungenügender Evidenz für die Zielpopulation dieser Leitlinie und in Bezug auf einen Mortalitätsvorteil sowie mögliche zusätzliche Kosten einer parenteralen Ernährung in Abwesenheit eines klinischen Nutzens bevorzugt auch bei Patienten mit Sepsis und septischem Schock (nach hämodynamischer Stabilisierung) der frühzeitige Beginn einer enteralen Ernährung empfohlen, sofern nicht die oben genannten Kontraindikationen vorliegen [561, $566,572,577]$.

\section{T.2 | Empfehlung | 2018}

\section{- Expertenkonsens \\ - Wir empfehlen eine frühzeitige minimale "trophische" enterale Ernährung möglichst innerhalb der ersten $48 \mathrm{~h}$ nach Beginn der Sepsis. \\ - Wir empfehlen, die enterale Ernäh- rung innerhalb der ersten 7 Tage entsprechend der metabolischen und gastrointestinalen Toleranz zu steigern. \\ - SSC-Leitlinienmodifikation \\ - Konsensstärke: $100 \%$}

Begründung. Die frühzeitige enterale Substratzufuhr bietet auch bei Patienten mit Sepsis potenzielle physiologische Vorteile für den Erhalt von Darmintegrität und intestinaler Barriere. Alles dies kann eine verminderte intestinale Permeabilität, Abschwächung der Entzündungsreaktion und Modulation der Stressreaktion mit reduzierter Insulinresistenz nach sich ziehen [578]. Um die Evidenz für diese Ernährungsstrategie zu beurteilen, ging die SSC-Arbeitsgruppe der Frage nach, ob eine frühzeitige kalorienbedarfsdeckende Ernährung, mit der innerhalb der ersten $48 \mathrm{~h}$ begonnen wird und bei der die Ernährungsziele innerhalb von $72 \mathrm{~h}$ nach der Aufnahme auf der ITS oder dem Trauma erreicht werden sollen, im Vergleich zu einem verzögerten Beginn der enteralen Zufuhr um mindestens $48 \mathrm{~h}$ das Ergebnis bei 
kritisch kranken Patienten verbessern würde.

In dem systematischen SSC-Review wurden insgesamt 11 Studien mit heterogenen, kritisch erkrankten Patientenpopulationen ( $n=412$ Patienten) eingeschlossen [579-589]. Nur eine Studie wurde spezifisch an Patienten mit Sepsis durchgeführt ( $n=43$ Patienten) [585]. Das Sterberisiko unterschied sich zwischen den Gruppen nicht signifikant (RR 0,75; $95 \%$-KI 0,43-1,31; $n=188 \mathrm{Pa-}$ tienten). Auch die Infektionsrate war nicht signifikant reduziert (RR 0,60; $95 \%$-KI 0,34-12,07; $n=122$ Patienten). Die anderen systematischen Reviews fokussierten sich auf Traumapatienten (3 Studien, 126 Patienten) bzw. heterogen zusammengesetzte Populationen von Intensivpatienten (6 Studien, $n=234 \mathrm{~Pa}$ tienten). Hier war eine frühzeitige enterale Ernährung mit einer geringeren Mortalität und Pneumonierate assoziiert [590, 591].

Im Gegensatz zu dem ersten systematischen Review beinhalteten die letztgenannten Reviews keine Studien, in denen die enterale Ernährung im Interventionsarm sowohl frühzeitig als auch kalorienbedarfsdeckend erfolgte und bei denen die Ernährung im Kontrollarm mindestens um $48 \mathrm{~h}$ verzögert wurde.

Außerdem wurde von der SSC-Arbeitsgruppe untersucht, ob ein frühzeitig hypokalorisches Ernährungsprotokoll (Beginn der enteralen Ernährung innerhalb der ersten $48 \mathrm{~h}$ und bis zu $70 \%$ der kalorischen Zielwerte über mindestens $48 \mathrm{~h}$ ) einer verzögerten enteralen Ernährungsstrategie überlegen war. In den beiden Studien, die diese Kriterien erfüllten, lagen keine statistischen Unterschiede in Bezug auf die Mortalität (RR 0,67; $95 \%$-KI 0,35-1,29; $n=229$; niedrige Evidenzqualität) oder Infektionsrate (RR 0,92; 95\%-KI 0,61-1,37; $n=229$; sehr niedrige Evidenzqualität) zwischen den Gruppen vor [592, 593]. Da es derzeit keine Evidenz für Nachteile einer frühzeitigen enteralen Ernährung im Vergleich $\mathrm{zu}$ einem verzögerten Beginn gibt, wird aufgrund der pathophysiologischen Konzepte zum Erhalt der intestinalen Barriere eine schwache Empfehlung für einen frühzeitigen Beginn der Ernährung bei Patienten mit
Sepsis ausgesprochen. Nach hämodynamischer Stabilisierung des Patienten im septischen Schock (z. B. stabile Katecholamingabe, Laktatnormalisierung) können ebenso minimale Zufuhrraten $(5-10 \mathrm{ml} / \mathrm{h})$ einer enteralen Ernährung unter engmaschigem Monitoring der gastrointestinalen und metabolischen Toleranz verabreicht werden (Kontraindikationen s. oben).

Biologische Grundlage für eine trophische oder hypokalorische Ernährung ist die aus der reduzierten Kalorienzufuhr potenziell resultierende Stimulation der Autophagie, die als Verteidigungsund Reparaturmechanismus auch zu einer Reduzierung des Infektionsrisikos führen könnte. Die SSC-Arbeitsgruppe hat als trophische/hypokalorische Ernährung ein Kalorienziel von $70 \%$ oder weniger mindestens für einen Zeitraum von $48 \mathrm{~h}$ definiert, bevor eine weitere Steigerung erfolgt. Das systematische Review identifizierte 7 randomisierte Studien mit 2665 untersuchten Patienten [594-601]. Die Patientenpopulationen umfassten heterogene Gruppen kritisch kranker Patienten. Unterernährte Patienten wurden von 4 der Studien ausgeschlossen und der durchschnittliche Body-MassIndex in den verbleibenden 3 Studien lag zwischen 28 und 30. Die Ziele für die trophische-/hypokalorische Ernährung lagen im Bereich von $10-20 \mathrm{kcal}$ pro Stunde bis zu $70 \%$ des anvisierten Ziels. Die Studienintervention umfasste eine Zeitdauer von 6-14 Tagen (oder den Zeitraum bis zur Entlassung von der ITS). In 3 der Studien wurde zur Deckung des Proteinbedarfs 0,8-1,5 g/kg und Tag verabreicht. Insgesamt bestanden zwischen den Studiengruppen keine Unterschiede in Bezug auf die Letalität (RR 0,95; $95 \%$ KI $0,82-1,10$; $n=2665$; hohe Evidenzqualität), die Infektionsrate (RR 0,96; 95\%-KI 0,83-1,12; $n=2667$; mittlere Evidenzqualität) oder die Aufenthaltsdauer auf der ITS (MD, -0,27 Tage; $95 \%$ $\mathrm{KI}-1,40$ bis 0,86 ; $n=2567$; mittlere Evidenzqualität). Eine randomisierte Studie zur hypokalorischen Ernährung (anvisiertes Ernährungsziel 40-60\% über einen Zeitraum von bis zu 14 Tagen) enthielt eine Untergruppe von 292 Sepsispatienten; hier wurden ebenfalls keine Unterschiede in Bezug auf den Tod nach
Ablauf von 90 Tagen zwischen den Studiengruppen festgestellt (RR 0,95; $95 \%$ KI 0,71-1,27; $p=0,82$ für Interaktion). Eine weitere systematische Übersicht zur normo- vs. hypokalorischen Ernährung ergab ebenfalls zwischen den Studiengruppen keine Unterschiede in Bezug auf Krankenhausletalität, Infektionen, Intensivaufenthaltsdauer und beatmungsfreie Tage [595].

Eine Studie zur trophischen/hypokalorischen Ernährung an 525 Patienten, bei der lediglich $20 \%$ des Kalorienziels in einem Zeitraum von bis zu 6 Tagen verabreicht wurden, ergab keinen Unterschied in Bezug auf die Muskelstärke, die Muskelmasse und einen 6-MinutenGehtest nach Ablauf von 6 Monaten oder einem Jahr. Bei den Patienten in der trophischen/hypokalorischen Ernährungsgruppe war jedoch die Wahrscheinlichkeit erhöht, innerhalb der ersten 12 Monate der Nachuntersuchungsphase eine Rehabilitationsbehandlung $\mathrm{zu}$ benötigen [602].

Tian et al. führten eine Metaanalyse von 8 kontrollierten Studien mit 1895 Intensivpatienten zur Frage der Höhe der enteralen Kalorienzufuhr durch [574]. Keine Unterschiede fanden sich zwischen einer niedrigen und hohen Energiezufuhr für die Parameter Mortalität, Infektionsrate und gastrointestinale Intoleranz. In einer Subgruppe von $\mathrm{Pa}$ tienten mit niedriger Energiezufuhr von 33,3-66,6\% des Energieziels zeigte sich im Vergleich zur höheren Energiezufuhr $>66,6 \%$ eine niedrigere Mortalität (RR $0,68 ; 95 \%$-KI $0,51-0,82 ; p=0,01)$ und gastrointestinale Intoleranz (RR 0,65 ; 95\%-KI 0,43-0,99; $p=0,05)$. Hieraus wurde geschlossen, dass die hohe enterale Energiezufuhr bei Intensivpatienten ohne Mangelernährung keine Vorteile bietet und das Risiko für gastrointestinale Komplikationen erhöht. Zur Frage der nahe am Kalorienziel orientierten Energiezufuhr ergab eine Metaanalyse von 10 randomisierten Studien mit 3155 Teilnehmern keine Vorteile (RR 1,02; $95 \%$-KI 0,81-1,27; $p=0,89$; [603]).

Zusammenfassend spricht die aktuelle Evidenz mehr für eine trophisch beginnende hypokalorische enterale Ernährung als für ein frühzeitiges „Erzwingen“ einer kalorienbedarfsdeckenden Ernäh- 
rung $[558,604]$. Die enterale Kalorienzufuhr sollte insbesondere in der Akutphase der Sepsis nach individueller intestinaler und metabolischer Toleranz gesteigert werden.

\section{T.3a | Empfehlung | 2018}

\section{- Expertenkonsens \\ - Wir empfehlen eine kombiniert ente- ral/parenterale Ernährung innerhalb der ersten 7 Tage, sofern eine ziel- orientierte und an die individuelle Toleranz adaptierte Steigerung der enteralen Ernährung nicht möglich ist. \\ - Zusätzliche DSG-Leitlinienempfeh- lung \\ - Konsensstärke: $100 \%$}

\section{T.3b | Empfehlung | 2018}

\section{- Expertenkonsens \\ - Bei Kontraindikationen für eine en- terale Ernährung empfehlen wir den frühzeitigen Einsatz einer an die individuelle metabolische Tole- ranz adaptierten rein parenteralen Ernährung. \\ - Zusätzliche DSG-Leitlinienempfeh- lung \\ - Konsensstärke: $100 \%$}

Begründung. Bei einigen Patienten mit Sepsis oder septischem Schock kann eine frühzeitige enterale Ernährung nur eingeschränkt realisierbar sein, was auf eine Dysfunktion des Gastrointestinaltrakts z. B. im Zusammenhang mit chirurgischen Eingriffen oder eine gastrointestinale Intoleranz zurückzuführen ist. Bei dieser Untergruppe muss diskutiert werden, ob die parenterale Ernährung frühzeitig mit oder ohne enterale Ernährung zugeführt werden sollte, um das Kalorienziel zu erreichen, ob ausschließlich eine trophische/hypokalorische enterale Ernährung erfolgen sollte oder ob überhaupt keine Nahrung verabreicht werden sollte mit Ausnahme von intravenöser Glukose zur Kalorienbereitstellung.

Um dieser Frage nachzugehen, wurde ein systematisches SSC-Review durchgeführt, in den insgesamt 4 Studien mit 6087 Patienten einbezogen wurden [605-608]. Zwei der einbezogenen Studien beinhalteten 98,5\% der Patienten im Rahmen des Review, und bei diesen Studien handelte es sich bei mehr als $65 \%$ der Patienten um chirurgische, kritisch erkrankte Patienten [606, 609]. Nur $20 \%$ der Patienten waren als septisch zu betrachten. Unterernährte Patienten wurden entweder ausgeschlossen oder repräsentierten einen sehr kleinen Anteil $(n=46,3,3 \%)$. In 3 der Studien wurde eine parenterale Ernährung angesetzt, wenn die enterale Ernährung nach den ersten 7 Behandlungstagen nicht vertragen wurde [606, 607, 609]. Das SSC-Review ergab, dass eine frühzeitige parenterale Ernährung mit oder ohne Supplementierung durch eine enterale Ernährung nicht mit einer reduzierten Mortalität verbunden war (RR 0,96; 95\%-KI 0,79-1,16; $n=6087$; mittlere Evidenzqualität), sondern mit einem erhöhten Infektionsrisiko (RR 1,12; $95 \%$ KI, 1,02-1,24; 3 Studien; $n=6054$; mittlere Evidenzqualität). Die Ergebnisse in Bezug auf die Beatmungsdauer wurden divergierend in den 2 großen Studien berichtet, wobei eine Studie einen Anstieg suggerierte [609] und die andere einen Rückgang der Beatmungszeit im Zusammenhang mit einer frühzeitigen parenteralen Ernährung berichtete [606]. Eine Studie berichtete auch bei Anwendung des Subjective Global Assessment Score über eine geringere Rate an Muskelatrophie und Fettverlust in der Gruppe mit frühzeitiger parenteraler Ernährung [606]. Die Erhöhung der Energiedichte einer standardenteralen Ernährung von 1 auf $1,5 \mathrm{kcal} / \mathrm{ml}$ hat in einer großen randomisierten Studie bei mechanisch beatmeten Intensivpatienten keine Vorteile bezüglich des Outcomes ergeben [610].

Eine durch indirekte Kalorimetrie und Messung der Harnstoffausscheidung im Urin individuell gestaltete („goal-directed“) Kalorienzufuhr hat in einer randomisierten Studie (EAT-ICU-Trial) von 199 beatmeten Intensivpatienten mit einem Aufenthalt von mehr als 3 Tagen im Vergleich mit einer Standardernährung von $25 \mathrm{kcal} / \mathrm{kg}$ und Tag keine Vorteile bezüglich der Outcomeparameter sowie der Lebensqualität nach 6 Monaten (PCS Score, SF-36) erbracht [611].

Die SSC-Arbeitsgruppe hatte insgesamt festgestellt, dass aktuell keine klare Evidenz für den allgemeinen Beginn einer frühzeitigen parenteralen Ernährung (innerhalb der ersten 7 Behandlungstage) bei Patienten mit Kontraindikationen oder Unverträglichkeit für die enterale Ernährung vorliegt. Fehlendem klinischem Vorteil bezüglich der Letalität stehen ein eher erhöhtes Infektionsrisiko und zusätzliche Kosten der parenteralen Ernährung gegenüber. Diese Überlegungen führten die SSC-Arbeitsgruppe zu folgender Empfehlung. „Wir empfehlen, von der Verabreichung einer rein parenteralen Ernährung oder einer parenteralen Ernährung in Kombination mit enteraler Ernährung in den ersten 7 Tagen bei kritisch erkrankten Patienten mit Sepsis oder septischem Schock abzusehen. Sofern eine frühzeitige minimale und trophische enterale Ernährung nicht möglich ist, empfehlen wir bevorzugt die intravenöse Verabreichung von Glukose“.

Weitere klinische Studien zur Charakterisierung geeigneter Untergruppen für eine frühzeitige parenterale Ernährung wurden für notwendig erachtet. Hierbei sollte der Fokus auf bereits bei Aufnahme auf die ITS unterernährte Patienten gelegt werden, die bisher in den meisten klinischen Studien ausgeschlossen wurden.

Drei seit der Veröffentlichung der SSC Leitlinie publizierte Metaanalysen [574, $604,612]$ zeigen, dass $<33 \%$ Zielkalorien (im Vergleich $\mathrm{zu}>33 \%$ ) in der Akutphase mit einer erhöhten Mortalität assoziiert sind. Dieser Effekt war in der Metaanalyse von Choi et al. auch unabhängig vom BMI. Weitere aktuelle Daten bestätigen die negativen Auswirkungen eines kumulativen Kaloriendefizits beim kritisch Kranken [613-617] und haben die DSG-Arbeitsgruppe im Expertenkonsens und Einklang mit den aktuellen DGEM- und ESPEN-Leitlinien zur Modifikation der Empfehlung veranlasst. Hierbei wird der Patient mit Sepsis oder septischem Schock primär als ein metabolischer Hochrisikopatient angesehen [557, 618]. 
So schlagen wir unter Berücksichtigung der methodischen Schwächen der in der SSC-Leitlinie angeführten RCT und zuvor genannter aktuellen Metaanalysen eine kombinierte enteral/ parenterale Ernährung innerhalb der ersten 7 Tage nach Beginn der Sepsis vor, sofern eine zielorientierte und an die individuelle Toleranz adaptierte Steigerung der enteralen Ernährung nicht ausreichend möglich ist.

Die aktuelle ASPEN-Leitlinie [618] empfiehlt den frühzeitigen Beginn einer rein parenteralen Ernährung primär bei mangelernährten Patienten und solchen mit metabolischem Risiko (NRS $>5$ oder NUTRIC-Score $>5$ ). Die aktualisierte ESPEN-Leitlinie [559] empfiehlt im Fall einer Kontraindikation zur oralen/ enteralen Ernährung den Beginn einer parenteralen Ernährung innerhalb von 3-7 Tagen. Die aktuelle DGEM-Leitlinie empfiehlt, bei Vorliegen von Kontraindikationen für eine enterale Ernährung eine parenterale Ernährung durchzuführen, um die an die Phase der Erkrankung und an die individuelle metabolische Toleranz angepasste Kalorien- bzw. Proteinzufuhrrate zu erreichen. Auch kann insbesondere bei vorbestehender Mangelernährung in der Akutphase frühzeitig eine (ggf. supplementäre) parenterale Ernährung zum Erreichen des Kalorien- und Proteinziels entsprechend der individuellen metabolischen Toleranz durchgeführt werden [557].

\section{T.4a | Empfehlung | 2018}

- Wir schlagen vor, von der routinemäBigen Überwachung des gastrischen Restvolumens bei kritisch erkrankten Patienten mit Sepsis oder septischem Schock abzusehen.

- SSC-Leitlinienadaptation

- Empfehlungsgrad: schwach

- Evidenzgrad: niedrig

\section{T.4b | Empfehlung | 2018}

- Wir schlagen jedoch Messungen des gastrischen Restvolumens bei Patienten mit gastraler Intoleranz und hohem Reflux sowie bei Patienten mit hohem Aspirationsrisiko vor.
- Bemerkungen: Dieser Vorschlag bezieht sich auf nichtchirurgische, kritisch erkrankte Patienten mit Sepsis oder septischem Schock.

- SSC-Leitlinienadaptation

- Konsensstärke bei Doppelabstimmung: $100 \%$

- Empfehlungsgrad: schwach

- Evidenzgrad: niedrig

Begründung. Kritisch kranke Patienten haben ein signifikantes Risiko für eine gastrointestinale Dysmotilität, was wiederum eine Prädisposition für Regurgitation oder Erbrechen, Aspiration und die Entwicklung einer Aspirationspneumonie darstellt. Der Zweck der Durchführung von Messungen des gastralen Residualvolumens (GRV) besteht in der Reduzierung des Risikos einer Aspirationspneumonie durch Anpassung der enteralen Zufuhrrate. Die Diskussion bezieht sich darauf, dass Beobachtungs- und Interventionsstudien nicht durchgängig eine Beziehung zwischen den GRV-Messungen (mit einer Schwelle von $200 \mathrm{ml}$ bis hin zur Nichtüberwachung des GRV) und den Refluxereignissen von Erbrechen, Aspiration oder Pneumonie nachgewiesen haben [619-623]. In dem systematischen SSC-Review wurde eine multizentrische Nichtunterlegenheitsstudie an 452 kritisch erkrankten Patienten identifiziert, die Patienten entweder in eine Gruppe ohne GRV-Messung oder in eine Gruppe mit GRV-Messung in 6-Stunden-Intervallen randomisierte [624]. Eine Ernährungsintoleranz wurde als Erbrechen in der Interventionsgruppe (ohne GRV-Messung) bzw. als GRV $>250 \mathrm{ml}$ bzw. Erbrechen oder beidem in der Kontrollgruppe (mit GRV-Messung) definiert. Obwohl Erbrechen häufiger (39,6\% vs. $27 \%$; mediane Differenz 12,6; $95 \%$-KI 5,4-19,9) in der Gruppe auftrat, in der keine GRV-Messung erfolgte, war diese Strategie gegenüber der 6-stündlichen Messung des GRV nicht unterlegen, da kein Unterschied in der Häufigkeit der VAP (primärer Endpunkt) bestand (16,7\% vs. 15,8\%; Differenz 0,9\%; $95 \%$ KI $-4,8$ bis $6,7 \%$ ). Ebenfalls kein Unterschied zwischen beiden Gruppen zeigte sich bezüglich Mortalität nach 28 und
90 Tagen. Patienten, bei denen innerhalb von 4 Wochen vor Studienbeginn ein chirurgischer Eingriff vorgenommen worden war, wurden in diese Studie nicht eingeschlossen. Daher können diese Ergebnisse nicht zwangsläufig auf chirurgische Patienten angewendet werden. Die Ergebnisse dieser Studie stellen jedoch den Bedarf für routinemäßige GRV-Messungen als Methode zur Reduzierung von Aspirationspneumonien infrage. Dies gilt vor allem für Patienten mit niedrigem Aspirationsrisiko. Aufgrund dieser Daten und der möglichen Einsparung der zur Messung erforderlichen Pflegeressourcen sprechen wir eine Empfehlung gegen die routinemäßige GRV-Überwachung bei allen Sepsispatienten aus, sofern keine manifeste Ernährungsintoleranz vorliegt (z. B. Erbrechen, Reflux von Nahrung in die Mundhöhle) oder es sich um Patienten mit bekannt hohem Aspirationsrisiko (z.B. Z.n. chirurgischem Eingriff, hämodynamische Instabilität) handelt. Wir verweisen zusätzlich auf die S3-Leitlinie der DGEM „Besonderheiten der Überwachung bei künstlicher Ernährung“ [625].

\section{T.5 | Empfehlung | 2018}

\section{- Wir schlagen den Einsatz von Proki- netika bei kritisch kranken Patienten mit Sepsis oder septischem Schock und gastrointestinaler Intoleranz vor. - SSC-Leitlinienadaptation \\ - Konsensstärke: $100 \%$ \\ - Empfehlungsgrad: schwach \\ - Evidenzgrad: niedrig}

Begründung. Prokinetika, zu denen $\mathrm{Me}$ toclopramid, Domperidon und Erythromycin gehören, werden häufig auf der ITS eingesetzt. Jeder dieser Wirkstoffe verfügt über unterschiedliche pharmakodynamische und pharmakokinetische Eigenschaften, allerdings können alle diese Wirkstoffe mit einer Verlängerung des QT-Intervalls und ventrikulären Arrhythmien verbunden sein. Eine große Fallkontrollstudie an Patienten, die sich nicht auf der ITS befanden, demonstrierte eine 3-fache Zunahme des Risikos eines plötzlichen Herztods bei 
der Nutzung von Domperidon in einer Dosis von $>30 \mathrm{mg} / \mathrm{Tag}$ [626]. Eine andere retrospektive Kohortenstudie ergab, dass die Nutzung von Erythromycin durch ambulante Patienten mit einer 2-fachen Zunahme des Risikos eines plötzlichen Herztods verbunden war, insbesondere wenn das Medikament gleichzeitig mit anderen CYP3A-Inhibitoren verwendet wurde [627]. Der Einfluss auf ventrikuläre Arrhythmien bei kritisch kranken Patienten ist weniger klar.

Eine aktuelle systematische Übersicht und Metaanalyse von $13 \mathrm{RCT}$, in die 1341 kritisch kranke Patienten einbezogen wurden, ergab, dass die Nutzung von Prokinetika mit einem geringeren Risiko für eine enterale Ernährungsintoleranz assoziiert war (RR 0,73; 95\%KI 0,55-0,97; mittlere Evidenzqualität). Dies entsprach einer absoluten Risikoreduktion von $17 \%$. Die Nutzung von Prokinetika führte zu keiner signifikanten Erhöhung der Mortalität (RR 0,97; 95\%-KI 0,81-1,1; niedrige Evidenzqualität); nicht in allen Studien wurde die Inzidenz von tödlichen und nichttödlichen Herzrhythmusstörungen beobachtet. Es war kein signifikanter Effekt auf das Risiko für Pneumonie und Erbrechen festzustellen. Die Mehrheit der in dieser Metaanalyse inkludierten Studien untersuchte den Effekt von Metoclopramid oder Erythromycin; eine Subgruppenanalyse nach Medikamentenklasse war nicht aussagekräftig genug, um potenzielle Unterschiede festzustellen [628]. Die Überwachung des QT-Intervalls ist erforderlich, wenn diese Wirkstoffe auf der ITS eingesetzt werden, insbesondere, wenn sie gleichzeitig mit anderen, das QT-Intervall verlängernden Medikamenten verwendet werden [629]. Der Einsatz von Prokinetika sollte täglich im Hinblick auf Nutzen-Risiko-Relation und Indikation überprüft werden.

Zur Vermeidung von unerwünschten neurologischen Reaktionen wie Dyskinesien und Krämpfen wird seit Dezember 2013 von der regulierenden Behörde der European Medicines Agency (EMA) ein eingeschränkter Einsatz von Metoclopramid empfohlen: maximal 5 Tage mit Dosisreduktion bei Erwachsenen bis $30 \mathrm{mg} /$ Tag oder $0,5 \mathrm{mg} / \mathrm{kg}$ Körpergewicht und Tag unabhängig von der Applikati- onsart; keine längerfristige Anwendung bei Patienten mit chronischer Gastroparese. Die Effektivität von Metoclopramid zur Verstärkung der Magenmotilität und zur Verbesserung der enteralen Toleranz wird auch in den aktuellen DGEM- und ASPEN-Leitlinien betont. In der aktualisierten ESPEN-Leitlinie wird bei gastraler Intoleranz zunächst Erythromycin, dann entweder Metoclopramid oder eine Kombination beider Prokinetika empfohlen [559].

Unter Berücksichtigung der erwünschten Konsequenzen (geringeres Risiko einer Ernährungsintoleranz) und der niedrigen Evidenzqualität bei fehlendem Unterschied in Bezug auf die Mortalität oder Pneumonie wird eine schwache Empfehlung für die Verwendung der Prokinetika Metoclopramid oder Erythromycin zur Behandlung einer Ernährungsintoleranz bei Patienten mit Sepsis ausgesprochen. Weitere große vergleichende Studien werden benötigt, um die relative Wirksamkeit und Sicherheit verschiedener Prokinetika zu beurteilen und zu vergleichen. Wir verweisen zusätzlich auf die S3-Leitlinie „Besonderheiten der Überwachung bei künstlicher Ernährung“ [625].

\section{T.6 | Empfehlung | 2018}

\section{- Wir schlagen die Platzierung von postpylorischen Ernährungssonden bei kritisch erkrankten Patienten mit Sepsis oder septischem Schock mit gastraler Intoleranz sowie bei Pati- enten mit hohem Aspirationsrisiko vor. \\ - SSC-Leitlinienadaptation \\ - Konsensstärke bei Doppelabstim- mung: $100 \%$ \\ - Empfehlungsgrad: schwach \\ - Evidenzgrad: niedrig}

Begründung. Eine Ernährungsintoleranz wird als Erbrechen, abdominelle Distension oder hohes GRV definiert, die eine Unterbrechung der enteralen Ernährung nach sich ziehen. Kritisch kranke Patienten haben ein erhöhtes Risiko für eine gastrointestinale Motilitätsstörung. So bestehen bei etwa $50 \%$ der kritisch erkrankten Patienten
Zeichen einer verzögerten Magenentleerung, bei septischen Patienten sogar bis $\mathrm{zu} 80 \%$ [630, 690]. Eine Ernährungsintoleranz ist durch Erbrechen, die Aspiration von Mageninhalt oder sogar eine Pneumonie gekennzeichnet und kann eine Unterbrechung der enteralen Ernährung erfordern [631]. Die Pathophysiologie ist nicht vollständig geklärt und mit großer Wahrscheinlichkeit multifaktoriell. Die Gastroparese kann durch häufig in der Intensivmedizin verwendete Pharmaka begünstigt werden (z. B. Sedativa, Opioide oder NMBA) oder auch die Folge einer schockbedingten Minderperfusion im Gastrointestinaltrakt, einer Hyperglykämie oder der Gabe von Katecholaminen sein [632].

Bei einer Gastroparese bietet die postpylorische Sondenplatzierung theoretische Vorteile zur Verbesserung der Ernährungsintoleranz, da die Applikation der Nahrung unter Umgehung des Magens direkt in den Dünndarm ermöglicht wird. Postpylorische Ernährungssonden sind sicher, sind jedoch nicht immer verfügbar und erfordern besonderen technischen Aufwand und Expertise zur erfolgreichen Positionierung. Eine gastrische Luftinsufflation und Prokinetika sind jeweils effektive Hilfsmittel für die Platzierung [633]. Ferner kann die Positionierung endoskopisch erfolgen oder ein externes Magnetsystem zur Führung der Sonde verwendet werden. Eine systematische Übersichtsarbeit und eine Metaanalyse von randomisierten Studien untersuchten die Auswirkungen der postpylorischen (im Vergleich zur Magensonden-) Ernährung. Es wurden 21 RCT mit 1579 Patienten einbezogen. Die Ernährung über eine postpylorische Sonde reduzierte das Risiko einer Pneumonie im Vergleich zu einer Ernährung über Magensonden (RR 0,75; 95\%-KI 0,59-0,94; niedrige Evidenzqualität). Dies entspricht einer 2,5\%igen (95\%-KI 0,6-4,1\%) absoluten Reduzierung des Pneumonierisikos. Es wurde jedoch keine signifikante Auswirkung auf die Mortalität, die Aspiration oder das Erbrechen festgestellt. Dies steht im Einklang mit den Ergebnissen anderer Metaanalysen [634].

Obwohl die Nutzung von postpylorischen Sonden das Risiko einer Pneu- 
monie reduziert, ist die Evidenzqualität niedrig und der quantifizierbare Nutzen gering. Weitere Auswirkungen für den Patienten sind nicht sicher geklärt. Zur ökonomischen Bewertung der postpylorischen Sondenplatzierung besteht ein Bedarf für Kosten-Nutzen-Analysen. Daher ist bei Niedrigrisikopatienten die Indikation bei Abwägung zwischen erwünschten und unerwünschten Folgen relativ. Bei Patienten mit hohem Aspirationsrisiko (d.h. bei solchen mit wiederholter Aspiration in der Anamnese, einer schweren Gastroparese oder einer persistierenden Ernährungsintoleranz bei gastraler Ernährung) kann die postpylorische Sondenplatzierung gerechtfertigt sein.

Hier besteht Konsens mit den o.g. Leitlinien nationaler und internationaler Ernährungsgesellschaften.

\section{T.7 | Empfehlung | 2018}

\section{- Wir empfehlen, dass von der Verwen- dung von intravenösem Selen bei der Behandlung von Patienten mit Sepsis und septischem Schock abgesehen wird. \\ - SSC-Leitlinienadaptation \\ - Konsensstärke: $100 \%$ \\ - Empfehlungsgrad: stark \\ - Evidenzgrad: moderat}

Begründung. Selen wird mit der Intention verabreicht, eine Verminderung der Selenkonzentration im Rahmen der Sepsis zu korrigieren und einen positiven pharmakologischen Effekt auf die antioxidative Kapazität zu erzielen. Obwohl einige RCT verfügbar sind, ist die Evidenz für den Nutzen von intravenösem Selen nicht überzeugend. Zwei aktuelle Metaanalysen suggerieren (mit schwachen Ergebnissen) einen potenziellen Vorteil einer hochdosierten Selensupplementierung bei Sepsis [634, 635]. Die hochdosierte Gabe von Selen (Natriumselenit $1000 \mu \mathrm{g} / \mathrm{Tag}$ für max. 21 Tage) vs. Placebo wurde in einer multizentrischen randomisiert-kontrollierten Studie (SISPCT) an 1089 Patienten mit schwerer Sepsis oder septischem Schock neben einer prokalzitoningesteuerten Antibiotikatherapie untersucht [636].
In der „Intention-to-treat“-Analyse von 543 Patienten mit Selengabe vs. 546 mit Placebo zeigte sich im primären Endpunkt 28-Tage-Letalität mit 28,3\% (95\%-KI, 24,5-32,3\%) vs. 25,5\% (95\%KI 22,9-29,5\%) kein signifikanter Unterschied $(p=0,30$; [637]).

Des Weiteren wurden keine Unterschiede in Bezug auf die sekundären Endpunkte hinsichtlich des Auftretens einer nosokomialen Pneumonie oder der Aufenthaltsdauer auf der ITS festgestellt. Bei der Aktualisierung der SSC-Metaanalyse unter Einbeziehung der SISPCT-Studienergebnisse wurde kein Vorteil bezüglich der Mortalität bei hochdosierter Selengabe nachgewiesen. So kann die intravenöse Gabe von Selen bei septischen Patienten nicht empfohlen werden.

\section{T.8 | Empfehlung | 2018}

- Wir empfehlen, dass von der Verwendung von Glutamin bei der Behandlung von Patienten mit Sepsis oder septischem Schock abgesehen wird.

- SSC-Leitlinienadaptation

- Konsensstärke bei Doppelabstimmung: $100 \%$

- Empfehlungsgrad: stark

- Evidenzgrad: moderat

Begründung. Die Glutaminkonzentration im Plasma kann während einer kritischen Erkrankung reduziert sein. Plasmaglutaminspiegel sind bei nichtelektiven Aufnahmen auf die ITS signifikant niedriger als bei elektiven Patienten. Niedrige Glutaminspiegel sind Ausdruck der Schwere der Erkrankung und einer Infektion. Die exogene Supplementierung kann die Atrophie und Permeabilität der Darmschleimhaut verbessern und möglicherweise zu einer Reduktion der bakteriellen Translokation führen. $\mathrm{Zu}$ den anderen potenziellen Vorteilen gehören eine verbesserte Funktion von Immunzellen, eine verringerte proinflammatorische Zytokinproduktion und höhere Konzentrationen von Glutathion und damit eine erhöhte antioxidative Kapazität. Die klinische Signifikanz dieser pathophysiologischen Erkennt- nisse wurde jedoch noch nicht eindeutig festgestellt.

Obwohl eine frühere Metaanalyse eine Reduktion der Mortalität demonstrierte [638], war dies bei neueren Metaanalysen nicht der Fall [639-642]. Vier aktuelle gut konzipierte Studien wiesen in den Primäranalysen ebenfalls keinen Mortalitätsvorteil nach, wobei keine dieser Studien spezifisch auf septische Patienten fokussiert war [643-646]. In der Studie von Heyland und Mitarbeitern [646] war eine hochdosierte Glutaminsupplementierung bei Patienten mit Multiorganversagen (31\% der Patienten mit Sepsis als Diagnose) sogar mit einer signifikant höheren 6-Monats-Mortalität assoziiert. Zwei kleine Studien an septischen Patienten ergaben keinen Vorteil in Bezug auf die Mortalitätsraten [647, 648], demonstrierten aber eine signifikante Reduktion der Rate infektiöser Komplikationen und eine schnellere Erholung der Organfunktion.

Eine von der Arbeitsgruppe als qualitativ gut bewerteten „Umbrella“-Übersicht von 22 Metaanalysen sowie eine aktuelle hier noch nicht eingeschlossene Metaanalyse von 15 randomisierten Studien hat Vorteile für die Glutaminsupplementierung bei Intensivpatienten in Bezug auf die Rate infektiöser Komplikationen und der Krankenhausverweildauer gezeigt [649, 650]. In der Mehrheit der Metaanalysen wurde jedoch keine statistische Signifikanz erreicht. Hingewiesen worden ist auch auf die erhebliche und statistisch signifikante Heterogenität der Studien und Metaanalysen [649].

In der Zusammenschau der Daten für Intensivpatienten kann der Einsatz von Glutamin beim septischen Patienten mit Organdysfunktion nicht empfohlen werden.

\section{T.9 | Empfehlung | 2018}

- Wir empfehlen, dass von der Verwendung von Omega-3-Fettsäuren als Immunsupplement bei kritisch kranken Patienten mit Sepsis oder septischen Schock abgesehen wird (dies betrifft nicht den Einsatz Omega-3-fetthaltiger Lipidemulsionen im Rahmen der parenteralen Ernährung). 


\section{- SSC-Leitlinienadaptation \\ - Konsensstärke bei Doppelabstim- mung: $100 \%$ \\ - Empfehlungsgrad: stark \\ - Evidenzgrad: niedrig}

Begründung. Pathophysiologisch legt das hohe antiinflammatorische Potenzial durch den Shift der Mediatorsynthese die Gabe von Fischöl bzw. Omega-3Fettsäuren bei Intensivpatienten sogar mit Sepsis und septischem Schock nahe [651]. Andererseits kann eine bereits bestehende Immunsuppression zusätzlich verstärkt werden.

In den der SSC-Arbeitsgruppe vorliegenden systematischen Übersichten zur Omega-3-Supplementierung bei Intensivpatienten und auch solchen mit ARDS wurde ein therapeutischer Nutzen nicht sicher gezeigt. Eine große randomisierte kontrollierte Studie bei 272 Patienten mit Lungenversagen hatte sogar ein erhöhtes Risiko für Mortalität sowie verlängerte Beatmungs- und Intensivliegedauer ergeben [652].

In vielen Studien bei Intensivpatienten wurden vor allem enteral Omega-3Fettsäuren mit Vitaminen und Spurenelementen kombiniert, sodass die isolierte Beurteilung erschwert ist. Aus diesem Grund hat die SSC-Arbeitsgruppe eine Metaanalyse der Studien mit ausschließlicher Gabe von Omega-3-Fettsäuren im Interventionsarm vorgenommen. $16 \mathrm{Stu}-$ dien mit 1216 Patienten wurden eingeschlossen. Es gab keinen signifikanten Unterschied in der Letalität (RR 0,86 ; $95 \%$-KI 0,71-1,03; niedrige Evidenzqualität). Die Intensivliegedauer war jedoch bei Einsatz von Omega-3-Fettsäuren signifikant verkürzt (MD -3,84 Tage, 95\%KI, $-5,57$ bis $-2,12$; sehr niedrige Evidenzqualität). Insgesamt wurde die Evidenz als niedrig bewertet. Aufgrund der Unsicherheit über Nutzen und Nebenwirkungen wurde von der SSC-Arbeitsgruppe auch in Anbetracht der Kosten vom Einsatz außerhalb von randomisierten Studien stark abgeraten.

Die DSG-Arbeitsgruppe hat die seit 2016 erschienenen Studien und Metaanalysen mit Fokus auf Patienten mit Sepsis bewertet.
In einer aktuellen Metaanalyse der randomisiert-kontrollierten Studien mit septischen Patienten wurden 11 Studien mit 898 Patienten eingeschlossen. Hier wurde kein Einfluss der Ernährung mit Omega-3-Fettsäuren auf die Letalität (RR 0,$84 ; 95 \%$-KI 0,67-1,05; $p=0,17$ ) und die Rate infektiöser Komplikationen gezeigt (RR 0,95; $95 \%$-KI; 0,77-1,25; $p=0,70$ ). Signifikant reduziert war bei den mit Omega-3-Fettsäuren ernährten Patienten die Dauer der mechanischen Beatmung $(-3,82$; $95 \%$-KI $-4,61$ bis 3,04 ; $p<0,00001)$. Eine signifikante Heterogenität der Daten wurde für die Krankenhausverweil- und Intensivliegedauer festgestellt [692]. Eine weitere ebenfalls auf Patienten mit Sepsis fokussierte Metaanalyse von 17 Studien mit 1239 Patienten konnte für die Supplementierung mit Omega-3-Fettsäuren keinen signifikanten Effekt auf die Mortalität (RR 0,85; $95 \%$-KI $0,71-1,03, p=0,10 ; \mathrm{I}^{2}=0 \%$ ) beobachten, führte aber zu einer signifikanten Verkürzung des Intensivaufenthalts $(-3,79$ Tage; $95 \%-K I-5,49$ bis $-2,09$; $\left.p<0,0001 ; \mathrm{I}^{2}=82 \%\right)$ und Dauer der mechanischen Beatmung (-2,27 Tage; $95 \%$ KI $-4,27$ bis 0,$27 ; p=0,003 ; \mathrm{I}^{2}=60 \%$ ). Aufgrund der Heterogenität der Studien halten die Autoren selbst die Evidenz für nicht ausreichend, um eine Empfehlung zum Routineeinsatz beim septischen $\mathrm{Pa}$ tienten zu rechtfertigen [653, 691].

Für septische Patienten, die aufgrund einer schweren gastrointestinalen Dysfunktion nach den SSC-Leitlinien behandelt und ausschließlich parenteral ernährt wurden, haben Chen et al. in einer randomisierten verblindeten Pilotstudie bei Supplementierung mit $100 \mathrm{ml}$ Fischöl (10g Fischöl/Tag) eine signifikant niedrigere 28-Tage-Letalität in der Interventionsgruppe gezeigt $(12,5 \%$ vs. $41,7 \% ; p=0,023)$. Die verminderte Mortalität wurde von einem signifikant niedrigeren APACHE-II- und MarshallMultiorgandysfunktionsscore an Tag 7 $(p<0,05)$ sowie einer höheren Rate von T-Helfer-/-Inducer-Zellen und CD4/CD8-Lymphozyten $(p<0,01)$ begleitet [654].

Von derselben Arbeitsgruppe wurde in einer zweiten analog durchgeführten randomisierten Studie $(n=78)$ eine grenzwertig signifikant niedrige- re 60-Tage-Mortalität bei parenteraler Fischölsupplementierung gezeigt: $26,8 \%$ vs. $48,6 \%$ (OR 0,387; KI 0,150-0,996; $p=0,046)$. Die 28-Tage-Letalität war hier ohne Unterschied. Das Verhältnis von Omega-6 zu -3 betrug $5 \mathrm{zu} \mathrm{1.} \mathrm{In} \mathrm{der}$ multivariaten Analyse profitierten besonders Patienten mit hohem intraabdominellem Druck und intraabdomineller Sepsis, passend zu der früheren Beobachtungsstudie von Heller et al. (2006). Methodisch sieht die DSG-Arbeitsgruppe hier Schwächen. Nicht klar ist, ob es sich wirklich um 2 getrennte Studien handelt oder die Daten der Pilotstudie in der zweiten Studie enthalten sind. Auch die Definition der primären und sekundären Endpunkte ist nicht deutlich.

In einer prospektiven nichtrandomisierten, nach Auffassung der Arbeitsgruppe qualitativ guten Beobachtungsstudie (2 plus) bei 112 Patienten mit Sepsis war eine parenterale Supplementierung mit Omega-3-Fettsäuren mit einer signifikant geringeren Gesamtletalität (20 vs. $10 \% ; p=0,034)$ und Intensivverweildauer assoziiert. Keine Unterschiede wurden für TNF- $\alpha$ - und Prokalzitoninspiegel sowie die 28-TageMortalität, die Krankenhausverweildauer und die Dauer der mechanischen Beatmung beobachtet. Medikamentöse Nebenwirkungen wurden nicht beobachtet [655].

Bei der Gabe von Omega-3-Fettsäuren gilt ein Verhältnis von Omega-6 zu Omega-3 von 2,1 zu 1 als immunneutral. Die auf dem Markt verfügbaren Omega-3-haltigen Infusionslösungen berücksichtigen dieses Verhältnis, was eine Erklärung für die bisher nicht eindeutig gezeigten klinischen Vorteile bei Intensivpatienten sein kann, die zumeist erst in Metaanalysen gepoolter Daten herausgearbeitet wurden. Auch eine aktuelle produktspezifische von der Arbeitsgruppe als qualitativ gut bewertete Metaanalyse hat keine signifikanten Unterschiede gezeigt [656]. Insgesamt sind große multizentrische Studien auch zur Dosisfindung erforderlich.

In Zusammenschau der Heterogenität der Daten und im Einklang mit der aktuellen DGEM-S2k-Leitlinie kann die Empfehlung der SSC, bei septischen Patienten keine Omega-3-Fettsäuren 
einzusetzen, nur vor dem Hintergrund des „primum nil nocere“ (unter Nichtberücksichtung der positiven Daten) verstanden werden. Die DSG-Leitlinienkommission hat daher in der o.g. Empfehlung den Zusatz mit angeführt, dass die Nichtverwendung von Omega3-Fettsäuren als (hochdosiertes) Immunsupplement bei kritisch kranken Patienten mit Sepsis oder septischen Schock nicht den Einsatz Omega-3-fetthaltiger Lipidemulsionen im Rahmen der parenteralen Ernährung betrifft, da bisher keine schädlichen Wirkungen beschrieben wurden und klinische sowie experimentelle Studien mögliche Vorteile suggerieren. Auch die aktualisierte ESPEN-Leitlinie empfiehlt, dass enterale hochdosierte Omega-3-Nährlösungen nicht routinemäßig oder als Bolus zugeführt werden sollten, Omega3-Fettsäuren als ernährungsrelevanter Bestandteil enteraler Nährlösungen jedoch eingesetzt werden können. Bei Einsatz von parenteralen Lipidlösungen angereichert mit Fischöl können diese in einer Dosierung von $0,1-0,2 \mathrm{~g} / \mathrm{kg}$ und Tag verwendet werden [559].

\section{U. Setzen von Behandlungszielen}

\section{U.1 | Empfehlung | 2018}

\section{- Expertenkonsens \\ - Wir empfehlen, dass die Behand- lungsziele und die Prognose mit den Patienten und deren Angehörigen/ Betreuern besprochen werden. - SSC-Leitlinienadaptation - Konsensstärke: $100 \%$}

\section{U.2 | Empfehlung | 2018}

\section{- Wir empfehlen, dass die Behand- lungsziele in die Therapieplanung und gegebenenfalls Sterbebeglei- tung einbezogen werden, wobei geeignete palliative Behandlungs- prinzipien genutzt werden sollten. - SSC-Leitlinienadaptation \\ - Konsensstärke: $100 \%$ \\ - Empfehlungsgrad: stark \\ - Evidenzqualität: moderat}

Begründung. Die Sterblichkeitsrate von Sepsispatienten mit multiplem Organsystemversagen ist hoch und die Langzeitfolgen nach überlebter Sepsis sind häufig schwer. Das Ergebnis einer intensivmedizinischen Behandlung bei kritisch erkrankten Patienten akkurat vorherzusagen, ist problematisch. Dennoch ist die Aufstellung realistischer Therapieziele von außerordentlicher Wichtigkeit [657], insbesondere weil falsche Erwartungen bezüglich der Prognose bei den Angehörigen häufig vorkommen [658]. Eine nichtvorteilhafte, vollumfängliche, lebensverlängernde ITS-Behandlung steht nicht im Einklang mit dem Setzen von Behandlungszielen [659, 660]. Die Nutzung von Behandlungskonferenzen zur Identifizierung von Patientenverfügungen und von Behandlungszielen innerhalb von $72 \mathrm{~h}$ ab dem Zeitpunkt der Aufnahme auf der ITS fördert nachweislich die Kommunikation und die Verständigung zwischen der Familie des Patienten und dem Behandlungsteam, verbessert die Familienzufriedenheit, verringert Stress, Ängste und Depressionen bei den Verwandten, erleichtert die Entscheidungsfindung zur Sterbebegleitung und verkürzt die Aufenthaltsdauer auf ITS bei Patienten, die versterben [661, 662]. Die Förderung einer gemeinsamen Entscheidungsfindung mit dem Patienten und dessen Familie ist hilfreich bei der Sicherstellung einer angemessenen Versorgung auf der ITS und der Gewährleistung, dass überflüssige Behandlungsmaßnahmen vermieden werden $[663,664]$.

Die Palliativversorgung wird zunehmend als wesentlicher Bestandteil einer umfassenden Versorgung von kritisch erkrankten Patienten anerkannt, und zwar unabhängig von der Diagnose oder der Prognose [665]. Die Nutzung der Palliativmedizin auf ITS erweitert die Fähigkeiten zur Erkennung von Schmerzen und Ängsten, die Feststellung der Wünsche, Glaubensgrundsätze und Werte des Patienten und deren Einfluss auf die Entscheidungsfindung, die Entwicklung von flexiblen Kommunikationsstrategien, die Festlegung von Behandlungszielen, die Bereitschaft zur Sterbebegleitung durch Angehörige, die Unterstützung bei der Lösung von Konflikten innerhalb des
Teams und die Aufstellung von angemessenen Zielen für die lebenserhaltenden Maßnahmen [666].

Ein aktuelles systematisches Review von palliativen Maßnahmen und der Nutzung von vollumfänglichen Intensivbehandlungsplänen hat ergeben, dass Patienten, bei denen vollumfängliche Intensivbehandlungspläne und palliative Maßnahmen einbezogen wurden, trotz großer Abweichungen in Bezug auf den Studientyp und die Qualität in 9 randomisierten kontrollierten Studien und 13 nichtrandomisierten kontrollierten Studien durchgängig ein Muster aufwiesen, das auf einen Rückgang der ITS-Einweisungen und einen kürzeren Aufenthalt auf der ITS hindeutet [667].

Die signifikanten Abweichungen bei den Beurteilungen der einzelnen Krankenhäuser und der Bereitstellung von palliativen Maßnahmen stehen jedoch im Einklang mit früheren Studien, die Abweichungen bei der Versorgungsintensität am Lebensende nachgewiesen haben [668]. Trotz der Unterschiede in Bezug auf die geographische Lage, das Rechtssystem, die Religion und die Kultur besteht ein weltweiter professioneller Konsens im Hinblick auf die wesentlichen intensivmedizinischen Praktiken am Lebensende [669].

Der Förderung einer auf den Patienten und dessen Familie ausgerichteten Behandlung auf der ITS wird inzwischen ein hoher Stellenwert eingeräumt. Sie beinhaltet die Umsetzung von frühzeitigen und wiederholten Behandlungskonferenzen zur Reduzierung der Belastung der Familie und zur Verbesserung der Konsistenz in der Kommunikation sowie offene und flexible Besuchszeiten, die Anwesenheit der Familienmitglieder bei klinischen Visiten und die Einbeziehung von kulturellen und spirituellen Unterstützungsleistungen [393, 670-672].

\section{Spätfolgen der Sepsis}

Neben den im Rahmen validierter Testinstrumente (z. B. SF-36) erfassten Einschränkungen der gesundheitsbezogenen Lebensqualität [673-675] leidet eine Vielzahl von ehemaligen Sepsispatienten unter funktionellen Einschränkungen, die unter den Begriffen „critical illness 


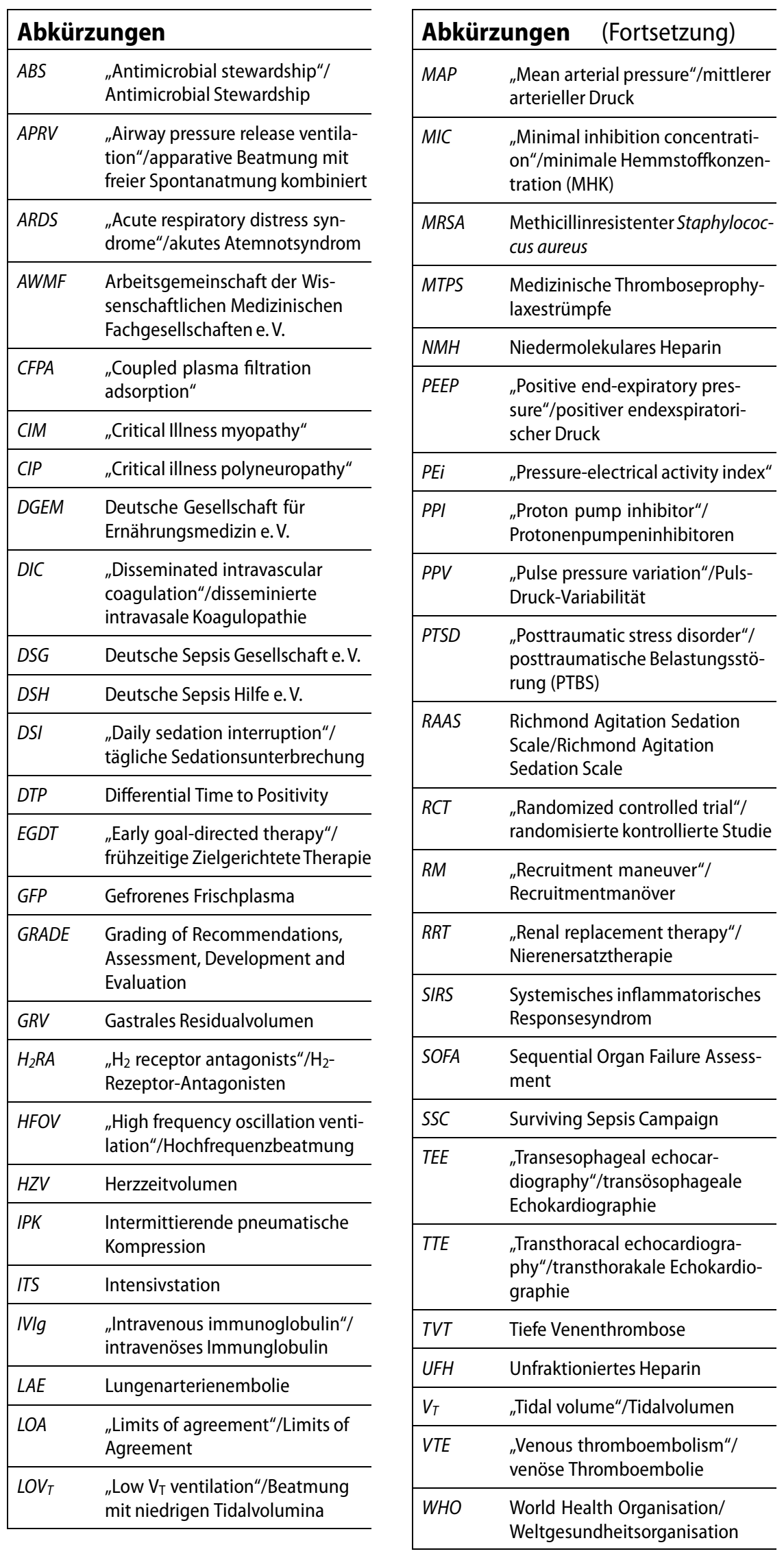

polyneuropathy“ (CIP) bzw. „critical illness myopathy“ (CIM) seit mehr als 2 Jahrzehnten bekannt sind [676]. Mehr als $70 \%$ der Patienten mit septischem Schock und mehr als $60 \%$ der mechanisch beatmeten Patienten sowie der Patienten mit einer schweren Sepsis zeigen signifikante elektrophysiologische Veränderungen bereits 3 Tage nach Aufnahme auf die ITS [677]. Assoziationen mit myopathischen oder neuropathischen Veränderungen zeigen neben der Sepsis und der Beatmung auch das Multiorganversagen, ARDS, eine systemische Inflammation, Kortikosteroide, Störungen des Glukosemetabolismus und die Liegedauer auf der ITS. In der Summe werden bei Patienten mit CIP/CIM häufiger Schwierigkeiten bei der Entwöhnung vom Beatmungsgerät („weaning failure“) und prolongierte Phasen der posthospitalen Rehabilitation beobachtet.

Zunehmend in den Blickpunkt geraten im Zusammenhang mit der perihospitalen funktionellen Entwicklung auch das Delirium während der Intensivtherapie sowie anhaltende neurokognitive Einschränkungen (ca. 17\%), posttraumatischer Disstress (PTSD, 44\%) und Depressionen (29\%; [678, 679, 682]). Der Grad der durch eine Sepsis resultierenden Funktionsdefizite und somit die tatsächliche Lebensqualität der Betroffenen kann jedoch durch eine geeignete Rehabilitationsmaßnahme durchaus beeinflussbar sein. Allerdings gibt es bis heute weder therapeutische Rehabilitationsstandards [680] noch auf diese Patienten ausgerichtete Rehabilitationseinrichtungen, da die zugrunde liegenden biologischen Mechanismen der Langzeitfolgen einer Sepsis nach intensivtherapeutischer Behandlung unzureichend verstanden sind. Außerdem sind die Langzeitfolgen den nachbehandelnden Ärzten in der Regel wenig bekannt. Bis zur Einführung der DRG wurden Sepsispatienten bis zu ihrer „Entlassungsfähigkeit“ in der Regel im Akutkrankenhaus versorgt, das jedoch im Allgemeinen nicht über entsprechende rehabilitationsmedizinische Ressourcen verfügt. Mit Einführung der DRG sehen sich diese Patienten jedoch mit einem weiteren Problem konfrontiert. Aufgrund zukünftig fehlender Abrechnungsgrundlagen ist das Akutkrankenhaus an 
einer vorzeitigen Entlassung des Patienten interessiert, um das pro Behandlungsfall zur Verfügung stehende Budget nicht über Gebühr zu überschreiten. Das Ergebnis ist, dass Sepsispatienten nun noch früher aus der akutmedizinischen Versorgung entlassen werden. Gezielte Forschung ist notwendig, um unser Verständnis der häufig lang andauernden neurokognitiven und motorischfunktionellen Beeinträchtigungen dieser Patientengruppe zu verbessern und mögliche Präventions- bzw. Therapieansätze aufzuzeigen [681, 682].

Es wird empfohlen, typische Sepsisfolgen - sofern möglich - bereits im akutmedizinischen Bereich zu erfassen und die nachbehandelnden Ärzte im postakuten und ambulanten Bereich über diesbezüglich bestehende bzw. potenziell im Langzeitverlauf auftretende Funktionsdefizite hinzuweisen.

\section{Korrespondenzadresse}

Prof. Dr. med. F. M. Brunkhorst

Zentrum für Klinische Studien, Integriertes Forschungs- und Behandlungszentrum (IFB) Sepsis und Sepsisfolgen, Klinik für Anästhesiologie und Intensivmedizin, Universitätsklinikum Jena

Am Klinikum 1, 07747 Jena, Deutschland frank.brunkhorst@med.uni-jena.de

Methodische Beratung und Begleitung. Arbeitsgemeinschaft der Wissenschaftlichen Medizinischen Fachgesellschaften e. V. (AWMF)

Leitlinienkommission. Prof. Dr. med. Frank Martin Brunkhorst (Vorsitz), Universitätsklinikum Jena

Prof. Dr. med. Markus Weigand (Stellvertretender Vorsitz), Universitätsklinikum Heidelberg

Arbeitsgruppen. Erstgenannt sind die Leiter der Autorengruppen.

Prävention: Prof. P. Gastmeier, Prof. S.W. Lemmen

Impfen:Prof. M. Pletz

A. Initiale hämodynamische Stabilisierung: Prof. A. Meier-Hellmann, Prof. M. Ragaller, Prof. A. Weyland, Prof. G. Marx, Prof. M. Bucher

B. Screening der Sepsis und Leistungsverbesserung: Prof. F.M. Brunkhorst, Prof. H. Gerlach

C. Diagnose: Prof. B. Salzberger, Dr. B. Grabein

D. Antimikrobielle Therapie: Prof. T. Welte, Prof

M. Pletz, Prof. M. Weigand, Dr. B. Grabein
E. Fokuskontrolle: Prof. T. Welte, Prof. M. Pletz, Prof. M. Weigand, Dr. B. Grabein

F. Flüssigkeitstherapie: Prof. A. Meier-Hellmann, Prof. M. Ragaller, Prof. A. Weyland, Prof. G. Marx, Prof. M. Bucher

G. Vasoaktive Medikation: Prof. A. Meier-Hellmann, Prof. M. Ragaller, Prof. A. Weyland, Prof. G. Marx, Prof. M. Bucher

H. Kortikosteroide: Prof. H. Gerlach, Prof. K. Werdan, Prof. S. Kluge, Prof. M. Weigand

I. Blutprodukte: Prof. H.-G. Bone

J. Immunglobuline: Prof. H. Gerlach, Prof. K. Werdan Prof. S. Kluge, Prof. M. Weigand

K. Blutreinigung: Prof. H. Gerlach, Prof. K. Werdan, Prof S. Kluge, Prof. M. Weigand

L. Antikoagulanzien: Prof. H. Gerlach, Prof. K. Werdan, Prof. S. Kluge, Prof. M. Weigand

M. Invasive Beatmung: Prof. C. Putensen, Prof. R. Rossaint, Prof. M. Quintel, Prof. S. Kluge

N. Sedierung und Analgesie: Prof. C. Spies, Dr. B. Weiß

O. Blutzuckerkontrolle: Prof. M. Weigand, Prof. H. Gerlach

P. Nierenersatztherapie: Prof. S. John, PD Dr. M. Oppert Prof. A. Jörres

Q. Bikarbonattherapie: Prof. A. Meier-Hellmann, Prof M. Ragaller, Prof. A. Weyland, Prof. G. Marx, Prof. M. Bucher

R. Prophylaxe einer venösen Thromboembolie: Prof. T. Brenner

S. Stressulkusprophylaxe: PD. G. Elke, Dr. M. Gründling,

T. Ernährung: Prof. K. Mayer; Prof. A. Weimann, PD. T.W. Felbinger, PD. G. Elke

U. Setzen von Behandlungszielen: Prof. F.M. Brunkhorst Prof. H. Gerlach

Spätfolgen der Sepsis: Prof. F.M. Brunkhorst, Prof. H. Axer

Weitere Mitwirkende. Methodik, Recherche und Evidenz: Cand. med. Nico Gagelmann

Prozessbegleitung: Dr. rer. medic. Susanne Blödt, Dr. med. Monika Nothacker (AWMF)

Leitliniensekretariat: Dr. rer. nat. Tabitha Heller, Dr. rer. medic. Wibke Wetzker
Einhaltung ethischer Richtlinien

Interessenkonflikt. Die ausführlichen Statements aller Autoren zum Interessenkonflikt finden Sie im elektronischen Zusatzmaterial.

Für diesen Beitrag wurden von den Autoren keine Studien an Menschen oder Tieren durchgeführt. Für die aufgeführten Studien gelten die jeweils dort angegebenen ethischen Richtlinien. 


\section{Anhang}

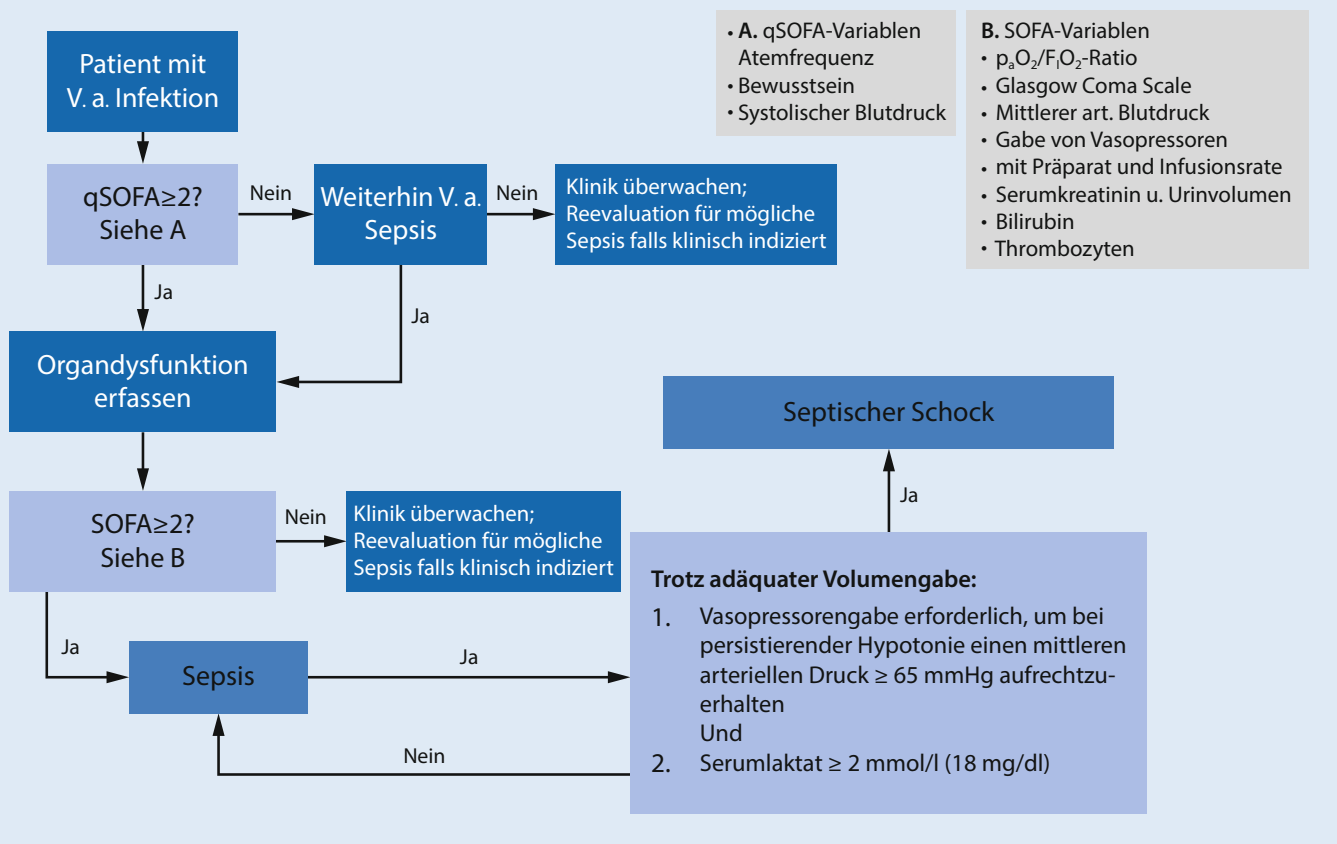

Abb. $1<$ Algorithmus bei Verdacht auf Infektion. (๑ F.M. Brunkhorst)

Tab. $1 \quad \mathrm{~F}_{1} \mathrm{O}_{2}$-PEEP-Tabelle 1 des ARDS-Network („nechanical ventilation protocol summary of low tidal volume used in the ALVEOLI study"), PEEP in $\mathrm{cm} \mathrm{H}_{2} \mathrm{O}$ [380]

\begin{tabular}{llllllll|lllllll}
$\mathrm{F}_{\mathrm{O}} \mathrm{O}_{2}$ & 0,3 & 0,4 & 0,4 & 0,5 & 0,5 & 0,6 & 0,7 & 0,7 & 0,7 & 0,8 & 0,9 & 0,9 & 0,9 & 1,0 \\
$\mathrm{PEEP}$ & 5 & 5 & 8 & 8 & 10 & 10 & 10 & 12 & 14 & 14 & 14 & 16 & 18 & $18-24$
\end{tabular}

Tab. $2 \quad \mathrm{~F}_{1} \mathrm{O}_{2}$-PEEP-Tabelle 2 des ARDS-Network (,nechanical ventilation protocol summary of low tidal volume used in the ALVEOLI study "), PEEP in $\mathrm{cm} \mathrm{H}_{2} \mathrm{O}$ [380]

\begin{tabular}{|c|c|c|c|c|c|c|c|c|c|c|c|c|c|c|}
\hline $\mathrm{F}_{1} \mathrm{O}_{2}$ & 0,3 & 0,3 & 0,3 & 0,3 & 0,3 & 0,4 & 0,4 & 0,5 & 0,5 & $0,5-0,8$ & 0,8 & 0,9 & 1,0 & 1,0 \\
\hline PEEP & 5 & 8 & 10 & 12 & 14 & 14 & 16 & 16 & 18 & 20 & 22 & 22 & 22 & 24 \\
\hline
\end{tabular}




\section{Literatur}

1. Singer M, Deutschman CS, Seymour CW et al (2016) The third international consensus definitions for sepsis and septic shock (sepsis-3). JAMA 315:801-810

2. Rhodes A, Evans LE, Alhazzani Wet al (2017) Surviving sepsis campaign: international guidelines for management of sepsis and septic shock: 2016. Crit Care Med 45(3):486-552

3. Levy MM, Fink MP, Marshall JC et al (2003) 2001 SCCM/ESICM/ACCP/ATS/SIS International Sepsis Definitions Conference. Crit Care Med 31(4):1250-1256

4. Shankar-Hari M, Phillips GS, Levy ML et al (2016) Sepsis definitions task force: developing a new definition and assessing new clinical criteria for septic shock: for the third international consensus definitions for sepsis and septic shock (sepsis-3). JAMA 315:775-787

5. Bone RC, Balk RA, Cerra FB et al (1992) Definitions for sepsis and organ failure and guidelines for the use of innovative therapies in sepsis. The ACCP/SCCM Consensus Conference Committee. American College of Chest Physicians/Society of Critical Care Medicine. Chest 101(6):1644-1655

6. Churpek MM, Zadravecz FJ, Winslow C, Howell MD, Edelson DP (2015) Incidence and prognostic value of the systemic inflammatory response syndrome and organ dysfunctions in ward patients. Am J Respir Crit Care Med 192:958-964

7. Kaukonen KM, Bailey M, Pilcher D, Cooper DJ, Bellomo R (2015) Systemic inflammatory response syndrome criteria in defining severe sepsis. N Engl J Med 372:1629-1638

8. Gaieski DF, Edwards JM, Kallan M, Carr BG (2013) Benchmarking the incidence and mortality of severe sepsis in the United States. Crit Care Med 41(5):1167-1174. https://doi.org/10.1097/CCM. 0b013e31827c09f8

9. Dellinger RP, Levy MM, Rhodes A et al (2013) Surviving sepsis campaign guidelines committee including the pediatric subgroup: surviving sepsis campaign: international guidelines for management of severe sepsis and septic shock, 2012. Intensive Care Med 39:165-228

10. Seymour CW, Liu VX, Iwashyna TJ et al (2016) Assessment of clinical criteria for sepsis: for the Third international consensus definitions for sepsis and septic shock (sepsis-3). JAMA 315:762-774. https://doi.org/10.1001/jama.2016.0288

11. Martin GS, Mannino DM, Eaton S et al (2003) The epidemiology of sepsis in the United States from 1979 through 2000. N Engl J Med 348:1546-1554

12. Storr J, Twyman A, Zingg W et al (2017) Core components for effective infection prevention and control programmes: new WHO evidencebased recommendations. Antimicrob Resist Infect Control 6:6. https://doi.org/10.1186/s13756-0160149-9

13. WHO (2014) WHO handbook for guideline development, 2. Aufl. World Health Organization, Geneva (https://www.who.int/publications/ guidelines/handbook_2nd_ed.pdf, accessed 16 October 2019)

14. Zingg W, Holmes A, Dettenkofer M et al (2015) Hospital organisation, management, and structure for prevention of health-care-associated infection: a systematic review and expert consensus. Lancet Infect Dis 15:212-224

15. Price L, MacDonald J, Melone L et al (2018) Effectiveness of national and subnational infection prevention and control interventions in high-income and upper-middle-income coun- tries: a systematic review. Lancet Infect Dis 18(5):e159-e171

16. Effective Practice and Organisation of Care (EPOC) (2015) Suggested risk of bias criteria for EPOC reviews. EPOC ressources for review authors. slo: Norwegian Knowledge Center for the Health Services. http://epoc.cochrane.org/sites/epoc. cochrane.org/files/public/uploads.pdf

17. Haley RW, Culver DH, White JW et al (1985) The efficacy of infection surveillance and control programs in preventing nosocomial infections in UShospitals. Am J Epidemiol 121:182-205

18. Mermel LA, Jefferson J, Blanchard $\mathrm{K}$ et al (2013) Reducing Clostridium difficile incidence, colectomies, and mortality in the hospital setting a successful multidisciplinary approach. Jt Comm J Qual Patient Saf 39:298-305

19. AWMF S3-Leitlinie Strategien zur Sicherung rationaler Antibiotika-Anwendung im Krankenhaus AWMF-Registernummer 092/001 update 2018. https://www.awmf.org/uploads/ tx_szleitlinien/092-001I_S3_Strategien-zurSicherung-rationaler-Antibiotika-Anwendung im-Krankenhaus_2019-04.pdf. Zugegriffen: 23. Nov. 2019

20. (2013) Surveillance of nosocomial infections as well as the detection of pathogens with special resistance and multi-resistance. Bundesgesundheitsblatt Gesundheitsforschung Gesundheitsschutz 56(4):580-583

21. Schwab F, Meyer E, Geffers C, Gastmeier P et al (2012) Understaffing, overcrowding, inappropriate nurse:ventilated patient ratio and nosocomial infections: which parameter is the best reflection of deficits? JHosp Infect 80(2):133-139

22. Birnbach DJ, Nevo I, Scheinman SR, Fitzpatrick $M$ Shekhterl, Lombard JL (2010) Patient safety begins with proper planning: a quantitative method to improve hospital design. Qual Saf Health Care 19:462-465

23. McLaws ML, Pantle AC, Fitzpatrick KR, Hughes CF (2009) Improvements in hand hygiene across New South Wales public hospitals: clean hands save lives, part III. Med J Aust 191(Suppl):S18-S24

24. Koff MD, Loftus RW, Burchman CC et al (2009) Reduction in intraoperative bacterial contamination of peripheral intravenous tubing through the use of a novel Device. Anesthesiology 110:978-985

25. Thomas BW, Berg-Copas GM, Vasquez DG, Jackson BL, Wetta-Hall R (2009) Conspicuous vs customary location of hand hygiene agent dispensers on alcohol-based hand hygiene product usage in an intensive care unit. J Am Osteopath Assoc 109:263-267

26. Whitby M, McLaws ML (2004) Handwashing in healthcare workers: accessibility of sink location does not improve compliance. J Hosp Infect 58:247-253

27. Jang JH, Wu S, Kirzner D et al (2010) Focus group study of hand hygiene practice among healthcare workers in a teaching hospital in Toronto, Canada. Infect Control Hosp Epidemiol 31:144-150

28. Händehygiene in Einrichtungen des Gesundheitswesens : Empfehlung der Kommission für Krankenhaushygiene und Infektionsprävention (KRINKO) beim Robert Koch-Institut (RKI). Bundesgesundheitsblatt, Gesundheitsforschung, Gesundheitsschutz. 2016;59(9):1189-220.

29. Engel C, Brunkhorst FM, Bone H-G et al (2007) Epidemiology of sepsis in Germany: results from a national prospective multicenter study. Intensive Care Med 33(4):606-618

30. von Baum H, Schweiger B, Welte T et al (2011) How deadly is seasonal influenza-associated pneumonia? The German Competence Network for Community-Acquired Pneumonia. Eur Respir J 37(5):1151-1157

31. DalhoffK, Abele-Horn M, Andreas Set al (2017) Epidemiologie, Diagnostikund Therapie erwachsener Patienten mit nosokomialer Pneumonie-Update

32. HagelS, Ludewig K, Moeser A etal (2016) Characteristics and management of patients with influenza in a German hospital during the 2014/2015 influenza season. Infection 44(5):667-672

33. Rivers E, Nguyen B, Havstad Setal (2001) Early goaldirected therapy collaborative group: early goaldirected therapy in the treatment of severe sepsis and septic shock. N Engl J Med 345:1368-1377

34. Peake SL, Delaney A, Bailey M et al (2014) Goaldirected resuscitation for patients with early septic shock. NEngl J Med 371(16):1496-1506

35. Yealy DM, Kellum JA, Huang DT et al (2014) A randomized trial of protocol-based care for early septic shock. NEngl J Med 370(18):1683-1693

36. Mouncey PR, Osborn TM, Power GS et al (2015) ProMlse trial investiga tors: trial of early, goaldirected resuscitation for septic shock. N Engl J Med 372:1301-1311

37. Levy MM, Dellinger RP, Townsend SR et al (2010) Surviving sepsis campaign: the surviving sepsis campaign: results of an international guideline-based performance improvement program targeting severe sepsis. Crit Care Med 38:367-374

38. Levy MM, Rhodes A, Phillips GS et al (2015) Surviving Sepsis Campaign: association between performance metrics and Outcomes in a 7.5-year study. Crit Care Med 43:3-12

39. Andrews B, Semler MW, Muchemwa L, Kelly P, LakhiS, HeimburgerDCetal (2017) Effect of an early resuscitation protocol on in-hospital mortality among adults with sepsis and hypotension. JAMA 318:1233

40. Leisman DE, Doerfler ME, Ward MF et al (2017) Survival benefit and cost savings from compliance with a simplified 3-Hour Sepsis Bundle in a Series of prospective, multisite, observational cohors. Crit Care Med 45:395-406

41. Seymour CW, Gesten F, Prescott HC et al (2017) Time to treatment and mortality during mandated emergency care for sepsis. N Engl J Med 376:2235-2244

42. Leisman DE, Goldman C, Doerfler ME et al (2017) Patterns and outcomes associated with timeliness of initial crystalloid resuscitation in a prospective sepsis and septic shock cohort. Crit Care Med 45:1596-1606

43. Boulain T, Garot D, Vignon P, Lascarrou JB, Desachy A, Botoc V, Follin A, Frat JP, Bellec F, Quenot JP, Mathonnet A, Dequin PF and for the Clinical Research in Intensive Care and Sepsis (CRICS) Group: Prevalence of low central venous oxygensat uration in the first hours of intensive care unit admission and associated mortality in septic shock patients: a prospective multicentre study. (2014) Dequin PF and for the Clinical Research in Intensive Care and Sepsis (CRICS) Group: prevalence of low central venous oxygensat uration in the first hours of intensive care unit admission and associated mortality in septic shock patients: a prospective multicentre study. Crit Care 18:609

44. DGAI - Deutsche Gesellschaft für Anästhesiologie und Intensivmedizin (2014) S3-Leitlinie Intravasale Volumentherapie beim Erwachsenen; Herausgeber: Stand 31.07.2014. https://www. awmf.org/uploads/tx_szleitlinien/001-0201_S3_ Intravasale_Volumentherapie_Erwachsenen_ 
2014-09-abgelaufen.pdf. Zugegriffen: 16. Jan. 2019

45. Cecconi M, De Backer D, Antonelli M et al (2014) Consensus on circulatory shockand hemodynamic monitoring. Task force of the European Society of Intensive Care Medicine. Intensive Care Med 40:1795-1815

46. American College of Cardiology Foundation Appropriate Use Criteria Task Force, ACCF/ASE/AHA/ ASNC/HFSA/HRS/SCAI/SCCM/SCCT/SCMR 2011 Appropriate Use Criteria for Echocardiography (2011) A Report of the American College of Cardiology Foundation Appropriate Use Criteria Task Force, American Society of Echocardiography American Heart Association, American Society of Nuclear Cardiology, Heart Failure Society of America, Heart Rhythm Society, Society for Cardiovascular Angiography and Interventions, Society of Critical Care Medicine, Society of Cardiovascular Computed Tomography, and Society for Cardiovascular Magnetic Resonance Endorsed by the American College of Chest Physicians. J Am Coll Cardiol 57:1126-1166

47. Eskesen TG, Wetterslev M, Perner A (2016) Systematic review including re-analyses of 1148 individual data sets of central venous pressure as a predictor of fluid responsiveness. Intensive Care Med 42:324-332

48. Monnet X, Marik P, Teboul JL (2016) Passive leg raising for predicting fluid responsiveness: a systematic review and meta-analysis. Intensive Care Med 42:1935-1947

49. FENICEInvestigators, ESICM Trial Group, Cecconi M, Hofer C, Teboul JL et al (2015) Fluid challenges in intensive care: the FENICEstudy: A globalinception cohort study. Intensive Care Med 41:1529-1537

50. S3-LL Intravasale Volumentherapie AWMF 2014; https://www.awmf.org/leitlinien/detail/II/001020.html

51. KelmDJ, Perrin JT, Cartin-Ceba R, Gajic O, SchenckL, Kenned CC (2015) Fluid overload in patients with severe sepsis and septic shock treated with early goal-directed therapy is associated with increased acute need for fluid-related medical interventions and hospital death. Shock 43:68-73

52. Boyd JH, Forbes J, Nakada T, Walley KR, Russell JA (2011) Fluid resuscitation in septic shock: A positive fluid balance and elevated central venous pressure are associated with increased mortality. Crit Care Med 39:259-265

53. Acheampong A, Vincent JL (2015) A positive fluid balance is an independent prognostic factor in patients with sepsis. Crit Care 16:251

54. Hjortrup PB, Haase N, Bundgaard H, Thomsen SL, Winding R, Pettilä V et al (2016) Restricting volumes of resuscitation fluid in adults with septic shock after initial management: the CLASSIC randomised, parallel-group, multicentre feasibility trial. Intensive Care Med 42:1695-1705

55. Hjortrup PB, Haas N, Wetterslev J, Lange T, Bundgaard H, Rasmussen BS, Dey N, Wilkman E, Christensen L, Lodahl D, Bestle M, Perner A (2017) Effects of fluid restriction on measures of circulatory efficacy in adults with septic shock. Acta Anaesthesiol Scand 61:390-398

56. LeDoux D, Astiz ME, Carpati CM et al (2000) Effects of perfusion pres sure on tissue perfusion in septic shock. Crit Care Med 28:2729-2732

57. Bourgoin A, Leone M, Delmas A et al (2005) Increasing mean arterial pres sure in patients with septic shock: effects on oxygen variables and renal function. Crit Care Med 33:780-786
58. Thooft A, Favory R, Salgado DR et al (2011) Effects of changes in arterial pressure on organ perfusion during septic shock. Crit Care 15:R222

59. SEPSISPAM Investigators, Asfar P, Meziani F, Hamel JF et al (2014) High versus low bloodpressure target in patients with septic shock. NEngl J Med 370:1583-1593

60. Canadian Critical Care Trials Group, Lamontagne $F_{\text {, }}$ Meade MO, Höbert PC et al (2016) Higher versus lower blood pressure targets for vaso pressor therapy in shock: a multicentre pilot randomized controlled trial. Intensive Care Med 42:542-550

61. Levy B (2006) Lactate and shock state: the metabolic view. Curr Opin Crit Care 12:315-321

62. Casserly B, Phillips GS, Schorr C et al (2015) Lactate measurements in sepsis-induced tissue hypoperfusion: results from the Surviving Sep sis Campaign database. Crit Care Med 43:567-573

63. LACTATE study group, Jansen TC, van Bommel J, Schoonderbeek FJ et al (2010) Early lactateguided therapy in intensive care unit patients: a multicenter, open-label, randomized controlled trial. Am J Respir Crit Care Med 182:752-761

64. Emergency Medicine Shock Research Network (EMShockNet) Investigators, Jones AE, Shapiro NI, Trzeciak S et al (2010) Lactate Clearance vs central venous oxygen saturation as goals of early sepsis therapy: a randomized clinical trial. JAMA 303:739-746

65. Lyu X, Xu Q, Cai G et al (2015) Efficacies of fluid resuscitation as guided by lactate Clearance rate and central venous oxygen saturation in patients with septic shock. Zhonghua Yi Xue Za Zhi 95:496-500

66. Tian HH, Han SS, Lv CJ et al (2012) The effect of early goal lactate clear ance rate on the Outcome of septic shock patients with severe pneumonia. Zhongguo Wei Zhong Bing Ji Jiu Yi Xue 24:42-45

67. Yu B, Tian HY, Hu ZJ et al (2013) Comparison of the effect of fluid resusci tation as guided either by lactate Clearance rate or by central venous oxygen saturation in patients with sepsis. Zhongguo Wei Zhong Bing Ji Jiu Yi Xue 25:578-583

68. Gu WJ, Zhang Z, Bakker J (2015) Early lactate Clearance-guided therapy in patients with sepsis: a meta-analysis with trial sequential analysis of randomized controlled trials. Intensive Care Med 41:1862-1863

69. Simpson SQ, Gaines M, Hussein $Y$ et al (2016) Early goal-directed therapy for severe sepsis and septic shock: a living systematic review. J Crit Care 36:43-48

70. Dellinger RP (2015) Foreword. The future of sepsis performance improve ment. Crit Care Med 43:1787-1789

71. Murphy DJ, Ogbu OC, Coopersmith CM (2015) ICU director data: using data to assess value, inform local change, and relate to the external world. Chest 147:1168-1178

72. Black MD, Schorr C, Levy MM (2012) Knowledge translation and the multi faceted intervention in the intensive care unit.CritCare Med 40:1324-1328

73. Gatewood MO, Wemple M, Greco S et al (2015) A quality improvement project to improve early sepsis care in the emergency department. BMJ Qual Saf 24:787-795

74. Hayden GE, Tuuri RE, Scott R et al (2016) Triage sepsis alert and sepsis protocol lower times to fluids and antibiotics in the ED. Am J Emerg Med 34(1):1-9

75. Jones SL, Ashton CM, Kiehne L et al (2015) Reductions in sepsis mortality and costs after design and implementation of a nurse-based early recognition and response program. JtComm J Qual Patient Saf 41:483-491

76. Damiani E, Donati A, Serafini G et al (2015) Effect of performance improve ment programs on compliance with sepsis bundles and mortality: a systematic review and meta-analysis of observational studies. PLoS ONE 10:e125827

77. Rhodes A, Phillips G, Beale R et al (2015) The surviving sepsis campaign bundles and outcome: results from the international multicentre prevalencestudy on sepsis (theIMpreSSstudy). Intensive Care Med 41:1620-1628

78. Zadroga R, Williams DN, Gottschall R et al (2013) Comparison of 2 blood culture media shows significant differences in bacterial recovery for patients on antimicrobial therapy. Clin Infect Dis 56:790-797

79. Kanegaye JT, Soliemanzadeh P, Bradley JS (2001) Lumbar puncture in pediatric bacterial meningitis: defining the time interval for recovery of cerebrospinal fluid pathogens after parenteral antibiotic pretreatment. Pediatrics 108:1169-1174

80. Pollack LA, van Santen KL, Weiner LM et al (2016) Antibiotic stewardship programs in U.S. acute care hospitals: findings from the 2014 national healthcare safety network annual hospital survey. Clin Infect Dis 63:443-449

81. Cardoso T, Carneiro AH, Ribeiro O et al (2010) Reducing mortality in severe sepsis with the implementation of a core 6-hour bundle: results from the Portuguese community-acquired sepsis study (SACiUCI study). Crit Care 14:R83

82. De Sousa AG, Fernandes JCJ, Santos GPD et al (2008) The impact of each action in the Surviving Sepsis Campaign measures on hospital mortality of patients with severe sepsis/septic shock. Einstein 6(3):323-327

83. Garnacho-Montero J, Gutiörrez-Pizarraya A, Escoresca-Ortega A et al (2013) De-escalation of empirical therapy is associated with lower mortality in patients with severe sepsis and septic shock. Intensive Care Med. https://doi.org/10. 1007/s00134-013-3077-7

84. Weiss CH, Persell SD, Wunderink RG et al (2012) Empiric antibiotic, mechanical ventilation, and central venous catheter duration as potential factors mediating the effect of a checklist prompting intervention on mortality: an exploratory analysis. BMC Health Serv Res 12:198

85. Edusepsis Study Group, Ferrer R, Artigas A, SuarezD et al (2009) Effectiveness of treatments for severe sepsis: a prospective, multicenter, observational study. Am J Respir Crit Care Med 180:861-866

86. Kumar A, Roberts D, Wood KE et al (2006) Duration of hypotension before initiation of effective antimicrobial therapy is the critical determinant of survival in human septic shock. Crit Care Med 34:1589-1596

87. Vaughn VM, Chopra V (2016) Revisiting the panculture. BMJ Qual Saf. https://doi.org/10.1136/ bmjqs-2015-004821

88. Weinstein MP, Reller LB, Murphy JR et al (1983) The clinical significance of positive blood cultures: a comprehensive analysis of 500 episodes of bacteremia and fungemia in adults. I. Laboratory and epidemiologic observations. Rev Infect Dis 5:35-53

89. Li J, Plorde JJ, Carlson LG (1994) Effects of volume and periodicity on blood cultures. J Clin Microbiol 32:2829-2831

90. Baron EJ, Miller JM, Weinstein MP et al (2013) A guide to utilization of the microbiology laboratory for diagnosis of infectious diseases: 2013 recommendations by the Infectious Diseases 
Society of America (IDSA) and the American Society for Microbiology (ASM)(a). Clin Infect Dis 57:e22-e121

91. O'Grady NP, Alexander M, Burns LA et al (2011) Healthcare Infection Control Practices Advisory Committee (HICPAC): guidelines for the prevention of intravascular catheter-related infections. Clin Infect Dis 52:e162-e193

92. Blot F, Schmidt E, Nitenberg G et al (1998) Earlier positivity of centralvenous- versus peripheralblood cultures is highly predictive of catheterrelated sepsis. J Clin Microbiol 36:105-109

93. Kaasch AJ, Rieg S, Hellmich M et al (2014) Differential time to positivity is not predictive for central line-related Staphylococcus aureus bloodstream infection in routine clinical care. JInfect 68:58-61

94. Malgrange VB, Escande MC, Theobald S (2001) Validity of earlier positivity of central venous blood cultures in comparison with peripheral blood cultures for diagnosing catheter-related bacteremia in cancer patients. J Clin Microbiol 39:274-278

95. O'Grady NP, Barie PS, Bartlett JG et al (2008) American College of Critical Care Medicine; Infectious Diseases Society of America: guidelines for evaluation of new fever in critically ill adult patients: 2008 update from the American College of Critical Care Medicine and the Infectious Diseases Society of America. Crit Care Med 36:1330-1349

96. Mermel LA, Allon M, Bouza E et al (2009) Clinical practice guidelines for the diagnosis and management of intravascular catheter-related infection: 2009 Update by the Infectious Diseases Society of America. Clin Infect Dis 49:1-45

97. Boyce JM, Nadeau J, Dumigan D et al (2013) Obtaining blood cultures by venipuncture versus from central lines: impact on blood culture contamination rates and potential effect on central line-associated bloodstream infection reporting. Infect Control Hosp Epidemiol 34:1042-1047

98. Beekmann SE, Diekema DJ, Huskins WC et al (2012) Infectious Diseases Society of America Emerging Infections Network: diagnosing and reporting of central line-associated bloodstream infections. Infect Control Hosp Epidemiol 33:875-882

99. Garcia RA, Spitzer ED, Beaudry J et al (2015) Multidisciplinary team review of best practices for collection and handling of blood cultures to determine effective interventions for increasing the yield of truepositive bacteremias, reducing contamination, and eliminating falsepositive central line-associated bloodstream infections. Am JInfect Control 43(11):1222-1237

100. Vincent JL, Brealey D, Libert N et al (2015) Rapid diagnosis of infections in the critically ill team: rapid diagnosis of infection in the critically ill, a multicenter study of molecular detection in bloodstream infections, pneumonia, and sterile site infections. Crit Care Med 43:2283-2291

101. Makristathis A, Riss S, Hirschl AM (2014) A novel fluorescence in situ hybridization test for rapid pathogen identification in positive blood cultures. Clin Microbiol Infect 20:0760-0763

102. Tissari P, Zumla A, Tarkka E et al (2010) Accurate and rapid identification of bacterial species from positive blood cultures with a DNA-based microarray platform: an observational study. Lancet 375:224-230

103. Niven DJ, Gaudet JE, Laupland KB et al (2015) Accuracy of peripheral thermometers for estimating temperature: a systematic review and metaanalysis. Ann Intern Med 163:768-779
104. Ferrer R, Martin-Loeches I, Phillips G et al (2014) Empiric antibiotic treat ment reduces mortality in severe sepsis and septic shock from the first hour: results from a guideline-based performance improvement program. Crit Care Med 42:1749-1755

105. Zhang D, Micek ST, Kollef MH (2015) Time to appropriate antibiotic therapy is an independent determinant of postinfection ICU and hospital lengths of stay in patients with sepsis. Crit Care Med 43:2133-2140

106. Cooperative Antimicrobial Therapy of Septic Shock (CATSS) Database Research Group, Bagshaw SM, Lapinsky S, Dial S et al (2009) Acute kidney injury in septic shock: clinical Outcomes and impact of dura tion of hypotension prior to initiation of antimicrobial therapy. Intensive Care Med 35:871-881

107. Iscimen R, Cartin-Ceba R, Yilmaz Met al (2008) Risk factors for the devel opment of acute lung injury in patients with septic shock: an observa tional cohort study. Crit Care Med 36:1518-1522

108. Garnacho-Montero J, Aldabo-Pallas T, GarnachoMontero C et al (2006) Timing of adequate antibiotic therapy is a greater determinant of out come than are TNF and IL-10 polymorphisms in patients with sepsis. Crit Care 10:R111

109. Barie PS, Hydo LJ, Shou J et al (2005) Influence of antibiotic therapy on mortality of critical surgical illness caused or complicated by infection. Surg Infect 6:41-54

110. Barochia AV, Cui X, Vitberg D et al (2010) Bundled care for septic shock: an analysis of clinical trials. Crit Care Med 38:668-678

111. Gaieski DF, Mikkelsen ME, Band RA et al (2010) Impact of time to antibiot ics on survival in patients with severe sepsis or septic shock in whom early goal-directed therapy was initiated in the emergency department. Crit Care Med 38:1045-1053

112. Kumar A (2016) Systematic bias in meta-analyses of time to antimicrobial in sepsis studies. Crit Care Med 44:e234-e235

113. Shirakura Y, Kuriyama A (2016) Timing of antibiotic administration in sepsis and septic shock: the impact that a meta-analysis does not depict. Crit Care Med 44:e1004

114. preSABATO study group, Kaasch AJ, Rieg S, Kuetscher J et al (2013) Delay in the administration of appropriate antimicrobial therapy in Staphy lococ cus aureus bloodstream infection: a prospective multicenter hospital-based cohort study. Infection 41:979-985

115. Corona A, Bertolini G, Lipman J et al (2010) Antibiotic use and impact on Outcome from bacteraemic critical illness: the BActeraemia Study in Intensive Care (BASIC). J Antimicrob Chemother 65:1276-1285

116. Giner AM, Kuster SP, Zbinden R et al (2011) Initial management of and Outcome in patients with pneumococcal bacteremia: a retrospec tive study at a Swiss university hospital, 2003-2009. Infection 39:519-526

117. Lin MY, Weinstein RA, Hota B (2008) Delay of active antimicrobial therapy and mortality among patients with bacteremia: impact of severe neu tropenia. Antimicrob Agents Chemother 52:3188-3194

118. Amaral AC, Fowler RA, Pinto R et al (2016) Cooperative antimicrobial therapy of septic shock database research group: patient and organizational factors associated with delays in antimicrobial therapy for septic shock. Crit Care Med 44:2145-2153
119. Funk DJ, Kumar A (2011) Antimicrobial therapy for life-threatening infections: speed is life. Crit Care Clin 27:53-76

120. Petitpas F, Guenezan J, Vendeuvre T et al (2016) Use of intra-osseous access in adults: a systematic review.Crit Care 20:102

121. Buck ML, Wiggins BS, Sesler JM (2007) Intraosseous drug administration in children and adults during cardiopulmonary resuscitation. Ann Pharmacother 41:1679-1686

122. Romanelli G, Cravarezza P (1995) Intramuscular meropenem in the treat ment of bacterial infections of the urinary and lower respiratory tracts. Italian Intramuscular Meropenem Study Group. JAntimicrob Chemother 36(Suppl A):109-119

123. Cormio L, Berardi B, Callea A et al (2002) Antimicrobial prophylaxis for transrectal prostatic biopsy: a prospective study of ciprofloxacin vs piperacillin/tazobactam. BJU Int 90:700-702

124. Barbhaiya RH, Knupp CA, Tenney J et al (1990) Safety, tolerance, and phar-macokinetics of cefepime administered intramuscularly to healthy subjects. J Clin Pharmacol 30:900-910

125. Kumar A, Ellis P, Arabi Y et al (2009) Cooperative Antimicrobial Therapy of Septic Shock Database Research Group: initiation of inappropri ate antimicrobial therapy results in a fivefold reduction of survival in human septic shock. Chest 136:1237-1248

126. Ibrahim EH, Sherman G, Ward S et al (2000) The influence of inadequate antimicrobial treatment of bloodstream infections on patient out comes in the ICU setting. Chest 118:146-155

127. Paul M, Shani V, Muchtar E et al (2010) Systematic review and meta-analy sis of the efficacy of appropriate empiric antibiotic therapy for sepsis. Antimicrob Agents Chemother 54:4851-4863

128. Kreger BE, Craven DE, McCabe WR (1980) Gramnegative bacteremia. IV. Re-evaluation of clinical features and treatment in 612 patients. Am J Med 68:344-355

129. Mermel LA, Maki DG (1993) Detection of bacteremia in adults: conse quences of culturing an inadequate volume of blood. Ann Intern Med 119:270-272

130. Bow EJ, Evans G, Fuller J et al (2010) Canadian clinical practice guidelines for invasive candidiasis in adults. Can J Infect Dis Med Microbiol 21:e122-e150

131. Connolly S (2011) Clinical practice guidelines: burn patient management. $\mathrm{ACl}$ statewide burn injury service. NSW Agency for Clinical Innovation, Chatswood

132. ESCMID Fungal Infection Study Group, Cornely OA, Bassetti M, Calandra T et al (2012) ESCMID* guideline for the diagnosis and manage ment of Candida diseases 2012: non-neutropenic adult patients. Clin Microbiol Infect 18(Suppl 7):19-37

133. Kalil AC, Metersky ML, Klompas M et al (2016) Management of adults with hospital-acquired and ventilator-associated pneumonia: 2016 clinical practice guidelines by the infectious diseases society of america and the American thoracic society. Clin Infect Dis 63:e61-e111

134. Liu C, Bayer A, Cosgrove SE et al (2011) Infectious Diseases Society of America: clinical practice guidelines by the infectious dis eases society of america for the treatment of methicillin-resistant Staphylococcus aureus infections in adults and children. Clin Infect Dis 52:e18-e55

135. Pappas PG, Kauffman CA, Andes DR et al (2016) Clinical practice guideline for the management of Candidiasis: 2016 update by the Infectious 
Diseases Society of America. Clin Infect Dis 62:e1-e50

136. Penack O, Becker C, Buchheidt D et al (2014) Management of sepsis in neutropenic patients: 2014 updated guidelines from the Infectious Diseases Working Party of the German Society of Hematology and Medical Oncology (AGIHO). Ann Hematol 93:1083-1095

137. German Society of Hematology and Oncology, Penack O, Buchheidt D, Christopeit M et al (2011) Management of sepsis in neutropenic patients: guidelines from the infectious diseases working party of the German Society of Hematology and Oncology. Ann Oncol 22:1019-1029

138. Solomkin JS, Mazuski JE, Bradley JS et al (2010) Diagnosis and management of complicated intraabdominal infection in adults and children: guide lines by the Surgical Infection Society and the Infectious Diseases Society of America. Surg Infect 11:79-109

139. Stevens DL, Bisno AL, Chambers HF et al (2014) Practice guidelines for the diagnosis and management of skin and soft tissue infections: 2014 update by the infectious diseases society of America. Clin Infect Dis 59:147-159

140. Micek ST, Welch EC, Khan J et al (2010) Empiric combination antibiotic therapy is associated with improved Outcome against sepsis due to Gram-negative bacteria: a retrospective analysis. Antimicrob Agents Chemother 54:1742-1748

141. Pittet D, Monod M, Suter PM et al (1994) Candida colonization and subsequent infections in critically ill surgical patients. Ann Surg 220:751-758

142. National Epidemiology of Mycoses Survey(NEMIS) Study Group, Blumberg HM, Jarvis WR, Soucie JM et al (2001) Risk factors for candidal bloodstream infections in surgical intensive care unit patients: the NEMIS prospective multicenter study. The National Epidemiology of Mycosis Survey. Clin Infect Dis 33:177-186

143. Green DL (2005) Selection of an empiric antibiotic regimen for hospital acquired pneumonia using a unit and culture-type specific antibio gram. JIntensive Care Med 20:296-301

144. Kaufman D, Haas CE, Edinger R et al (1998) Antibiotic susceptibility in the surgical intensive care unit compared with the hospital-wide antibio gram. Arch Surg 133:1041-1045

145. Kerremans JJ, Verbrugh HA, Vos MC (2012) Frequency of microbio logically correct antibiotic therapy increased by infectious disease consultations and microbiological results. J Clin Microbiol 50:2066-2068

146. Guo Y, Gao W, Yang H et al (2016) De-escalation of empiric antibiotics in patients with severe sepsis or septic shock: A meta-analysis. Heart Lung 45:454-459

147. Working Group IAP/APA (2013) Acute Pancreatitis Guidelines: IAP/APA evidence-based guidelines for the management of acute pancreatitis. Pancreatology 13(4):e1-e15

148. Wittau M, Mayer B, Scheele J et al (2011) Systematic review and meta analysis of antibiotic prophylaxis in severe acute pancreatitis. Scand J Gastroenterol 46:261-270

149. Avni T, Levcovich A, Ad-EIDDetal (2010) Prophylactic antibiotics for burns patients: systematic review and meta-analysis. BMJ 340:c241

150. Barajas-Nava LA, López-Alcalde J, Roquöi Figuls M, Solä I, Bonfill Cosp X (2013) Antibiotic prophylaxis for preventing burn wound infection. Cochrane Database Syst Rev. https://doi.org/10.1002/ 14651858.CD008738.pub2
151. Moore RD, Smith CR, Lietman PS (1984) Association of aminoglycoside plasma levels with therapeutic outcome in gram-negative pneumonia. Am J Med 77:657-662

152. Men P, Li HB, Zhai SD et al (2016) Association between the AUC0-24/MIC ratio of vancomycin and its clinical effectiveness: a systematic review and meta-analysis. PLoSONE 11:e146224

153. Moise-Broder PA, Forrest A, Birmingham MC et al (2004) Pharmacodynamics of vancomycin and other antimicrobials in patients with Staphylococ cus aureus lower respiratory tract infections. Clin Pharmacokinet 43:925-942

154. Cooperative Antimicrobial Therapy of Septic Shock-CATSS Database Research Group, ZelenitskyS, Rubinstein E, Ariano Retal (2013) Vancomycin pharmacodynamics and survival in patients with meth icillin-resistant Staphylococcus aureus-associated septic shock. Int J Antimicrob Agents 41:255-260

155. Forrest A, Nix DE, Ballow $\mathrm{CH}$ et al (1993) Pharmacodynamics of intra venous ciprofloxacin in seriously ill patients. Antimicrob Agents Chemother 37:1073-1081

156. Preston SL, Drusano GL, Berman AL et al (1998) Pharmacodynamics of levofloxacin: a new paradigm for early clinical trials. JAMA 279:125-129

157. Drusano GL, Preston SL, Fowler C et al (2004) Relationship between fluoroquinolone area under the curve: minimum inhibitory con centration ratio and the probability of eradication of the infecting pathogen, in patients with nosocomial pneumonia.J Infect Dis 189:1590-1597

158. Kashuba AD, Nafziger AN, Drusano GL et al (1999) Optimizing aminogly coside therapy for nosocomial pneumonia caused by gramnegative bacteria. Antimicrob Agents Chemother 43:623-629

159. Schentag JJ, Smith IL, Swanson DJ et al (1984) Role for dual individualiza tion with cefmenoxime. Am J Med 77:43-50

160. Crandon JL, Bulik CC, Kuti JL et al (2010) Clinical pharmacodynamics of cefepime in patients infected with pseudomonas aeruginosa. Antimicrob Agents Chemother 54:1111-1116

161. McKinnon PS, Paladino JA, Schentag JJ (2008) Evaluation of area under the inhibitory curve (AUIC) and time above the minimum inhibitory concentration (T)MIC) as predictors of Outcome for cefepime and ceftazidime in serious bacterial infections. Int J Antimicrob Agents 31:345-351

162. Roberts JA, Abdul-Aziz MH, Davis JS et al (2016) Continuous versus intermittent $\beta$-Lactam infusion in severe sepsis. A meta-analysis of individual patient data from randomized trials. Am J Respir Crit Care Med 194:681-691

163. Barza M, loannidis JP, Cappelleri JC et al (1996) Single or multiple daily doses of aminoglycosides: a meta-analysis. BMJ 312:338-345

164. Hatala R, Dinh T, Cook DJ (1996) Once-daily aminoglycoside dosing in immunocompetent adults: a meta-analysis. Ann Intern Med 124:717-725

165. van Zanten AR, Polderman $\mathrm{KH}$, van Geijlswijk IM et al (2008) Ciprofloxacin pharmacokinetics in critically ill patients: a prospective cohort study. J Crit Care 23:422-430

166. Zelenitsky SA, Ariano RE (2010) Support for higher ciprofloxacin AUC 24/MIC targets in treating Enterobacteriaceae bloodstream infection. JAntimicrob Chemother 65:1725-1732

167. Dunbar LM, Wunderink RG, Habib MP et al (2003) High-dose, short course levofloxacin for community-acquired pneumonia: a new treat ment paradigm. Clin Infect Dis 37:752-760
168. RybakMJ, Lomaestro BM, Rotschafer JC et al (2009) Vancomycin thera peutic guidelines: a summary of consensus recommendations from the infectious diseases Society of America, the American Society of Health-System Pharmacists, and the Society of Infectious Diseases Pharmacists. Clin Infect Dis 49:325-327

169. Matsumoto $\mathrm{K}$, Takesue $\mathrm{Y}$, Ohmagari $\mathrm{N}$ et al (2013) Practice guide lines for therapeutic drug monitoring of vancomycin: a consensus review of the Japanese Society of Chemotherapy and the Japanese Society of Therapeutic Drug Monitoring. JInfect Chemother 19:365-380

170. Steinmetz T, Eliakim-Raz N, Goldberg E et al (2015) Association of van comycin serum concentrations with efficacy in patients with MRSA infections: a systematic review and meta-analysis. Clin Microbiol Infect 21:665-673

171. Mohamed AF, Karaiskos I, Plachouras D et al (2012) Application of a load ing dose of colistin methanesulfonate in critically ill patients: popula tion pharmacokinetics, protein binding, and prediction of bacterial kill. Antimicrob Agents Chemother 56:4241-4249

172. Pea F, Brollo L, Viale P et al (2003) Teicoplanin therapeutic drug moni toring in critically ill patients: a retrospective study emphasizing the importance of a loading dose. J Antimicrob Chemother 51:971-975

173. Pea F, Viale $P$ (2009) Bench-to-bedside review: appropriate antibiotic ther apy in severe sepsis and septic shock - does the dose matter? Crit Care 13:214

174. Wang JT, Fang CT, Chen YC et al (2001) Necessity of a loading dose when using vancomycin in critically ill patients. J Antimicrob Chemother 47:246

175. Kumar A (2014) An alternate pathophysiologic paradigm of sepsis and sep tic shock: implications for optimizing antimicrobial therapy. Virulence 5:80-97

176. Rhodes NJ, MacVane SH, Kuti JLet al (2014) Impact of loading doses on the time to adequate predicted beta-lactam concentrations in prolonged and continuous infusion dosing schemes. Clin Infect Dis 59:905-907

177. Lodise TP Jr, Lomaestro B, Drusano GL (2007) Piperacillin-tazobactam for pseudomonas aeruginosa infection: clinical implications of an extendedinfusion dosing strategy. Clin InfectDis 44:357-363

178. Yost RJ, Cappelletty DM, RECEIPT Study group (2011) The retrospective cohort of extendedinfusion Piperacillin-Tazobactam (RECEIPT) study: a multicenterstudy.Pharmacotherapy31:767-775

179. Falagas ME, Tansarli GS, Ikawa Ket al (2013) Clinical Outcomes with extended or continuous versus short-term intravenous infusion of carbapenems and piperacillin/tazobactam: a systematic review and meta-analysis. Clin Infect Dis 56:272-282

180. Yusuf E, Spapen H, Piörard D (2014) Prolonged vs intermittent infusion of piperacillin/tazobactam in critically ill patients: a narrative and sys tematic review. J Crit Care 29:1089-1095

181. Mah GT, Mabasa VH, Chow l et al (2012) Evaluating Outcomes associated with alternative dosing strategies for piperacillin/tazobactam: a quali tative systematic review. Ann Pharmacother 46:265-275

182. Blot S, Koulenti D, Akova M et al (2014) Does contemporary vancomy cin dosing achieve therapeutic targets in a heterogeneous clinical cohort of critically ill patients? Data from the multinational DALI study. Crit Care 18:R99

183. DALI Study, Roberts JA, Paul SK, Akova M et al (2014) DALI: defining antibiotic levels in intensive 
care unit patients: are current $\beta$-lactam antibiotic doses sufficient for critically ill patients? Clin Infect Dis 58:1072-1083

184. Taccone FS, Laterre PF, Spapen $\mathrm{H}$ et al (2010) Revisiting the loading dose of amikacin for patients with severe sepsis and septic shock. Crit Care 14:R53

185. Rea RS, Capitano B, Bies R et al (2008) Suboptimal aminoglycoside dos ing in critically ill patients. Ther Drug Monit 30:674-681

186. Kumar A, Safdar N, Kethireddy S et al (2010) A survival benefit of combina tion antibiotic therapy for serious infections associated with sepsis and septic shock is contingent only on the risk of death a meta-ana lytic/meta-regression study. Crit Care Med 38:1651-1664

187. Kumar A, Zarychanski R, Light B et al (2010) Cooperative Antimicrobial Therapy of Septic Shock (CATSS) Database Research Group: Early combination antibiotic therapy yields improved survival compared with monotherapy in septic shock: a propensity-matched analysis. Crit Care Med 38:1773-1785

188. Al-Hasan MN, Wilson JW, Lahr BD et al (2009) Beta-lactam and fluoro quinolone combination antibiotic therapy for bacteremia caused by gramnegative bacilli. Antimicrob Agents Chemother 53:1386-1394

189. Delannoy PY, Boussekey N, Devos P et al (2012) Impact of combination therapy with aminoglycosides on the outcome of ICU-acquired bac teraemias. Eur J Clin Microbiol Infect Dis 31:2293-2299

190. Edusepsis Study Group, Díaz-Martín A, MartínezGonzález ML, Ferrer R et al (2012) Antibiotic prescription patterns in the empiric therapy of severe sepsis: combination of antimicrobials with different mecha nisms of action reduces mortality. Crit Care 16:R223

191. Martin-Loeches I, Lisboa T, Rodriguez A et al (2010) Combination antibi otic therapy with macrolides improves survival in intubated patients with community-acquired pneumonia. Intensive Care Med 36:612-620

192. German Study Group Competence Network Sepsis (SepNet), Brunkhorst FM, Oppert M, Marx G et al (2012) Effect of empirical treatment with moxifloxacin and meropenem vs meropenem on sepsis-related organ dysfunction in patients with severe sepsis: a randomized trial. JAMA 307:2390-2399

193. CAPUCI Study Group, Rodríguez A, Mendia A Sirvent JM et al (2007) Combination antibiotic therapy improves survival in patients with community-acquired pneumonia and shock. Crit Care Med 35:1493-1498

194. International Pneumococcal Study Group, Baddour LM, Yu VL, Klugman KP et al (2004) Combination antibiotic therapy lowers mortality among severely ill patients with pneumococcal bacteremia. Am JRespirCritCare Med 170:440-444

195. Hilf M, Yu VL, Sharp J et al (1989) Antibiotic therapy for pseudomonas aeruginosa bacteremia: outcome correlations in a prospective study of 200 patients. Am J Med 87:540-546

196. Tumbarello M, Viale P, Viscoli $C$ et al (2012) Predictors of mortality in bloodstream infections caused by Klebsiella pneumoniae carbapen emase-producing K. pneumoniae: importance of combination ther apy. Clin Infect Dis 55:943-950

197. Bass SN, Bauer SR, Neuner EA et al (2015) Impact of combination antimi crobial therapy on mortality risk for critically ill patients with carbape nem-resistant bacteremia. Antimicrob Agents Chemother 59:3748-3753

198. Poulikakos P, Tansarli GS, Falagas ME (2014) Combination antibiotic treatment versus monotherapy for multidrug-resistant, exten sively drugresistant, and pandrug-resistant Acinetobacter infec tions: a systematic review. Eur J Clin Microbiol Infect Dis 33:1675-1685

199. Falagas ME, Lourida P, Poulikakos P et al (2014) Antibiotic treatment of infections due to carbapenem-resistant enterobacteriaceae: sys tematic evaluation of the available evidence. Antimicrob Agents Chemother 58:654-663

200. Hu Y, Li L, Li W et al (2013) Combination antibiotic therapy versus mono therapy for pseudomonas aeruginosa bacteraemia: a meta-analysis of retrospective and prospective studies. Int $J$ Antimicrob Agents 42:492-496

201. Vardakas KZ, Tansarli GS, Bliziotis IA et al (2013) $\beta$-Lactam plus amino glycoside or fluoroquinolone combination versus $\beta$-lactam mono therapy for Pseudomonas aeruginosa infections: a metaanalysis. Int J Antimicrob Agents 41:301-310

202. Stevens DL, Tanner MH, Winship J et al (1989) Severe group A strepto coccal infections associated with a toxic shock-like syndrome and scarlet fever toxin A. NEngl J Med 321:1-7

203. Zimbelman J, Palmer A, Todd J (1999) Improved outcome of clindamy cin compared with betalactam antibiotic treatment for invasive Streptococcus pyogenes infection. Pediatr Infect Dis J 18:1096-1100

204. Safdar N, Handelsman J, Maki DG (2004) Does combination antimicrobial therapy reduce mortality in Gram-negative bacteraemia? A metaanalysis. Lancet Infect Dis 4:519-527

205. Paul M, Soares-Weiser K, Leibovici L (2003) Beta lactam monotherapy versus beta lactamaminoglycoside combination therapy for fever with neutropenia: systematic review and metaanalysis. BMJ 326:1111

206. Gomes Silva BN, Andriolo RB, Atallah AN, SalomäoR (2010) De-escalation of antimicrobial treatment for adults with sepsis, severe sepsis or septic shock. Cochrane Database Syst Rev. https://doi.org/10. 1002/14651858.CD007934.pub2

207. Morel J, Casoetto J, Jospö R et al (2010) Deescalation as part of a global strategy of empiric antibiotherapy management. $A$ retrospective study in a medico-surgical intensive care unit. Crit Care 14:R225

208. Joung MK, Lee JA, Moon SY et al (2011) Impact of de-escalation therapy on clinical Outcomes for intensive care unit-acquired pneumonia. Crit Care 15:R79

209. AZUREA Network Investigators, Leone M, Bechis C, Baumstarck K et al (2014) De-escalation versus continuation of empirical antimicrobial treatment in severe sepsis: a multicenter non-blinded randomized noninferiority trial. Intensive Care Med 40:1399-1408

210. Riccio LM, Popovsky KA, Hranjec T et al (2014) Association of excessive duration of antibiotic therapy for intra-abdominal infection with sub sequent extra-abdominal infection and death: a study of 2,552 con secutive infections. Surg Infect 15:417-424

211. Aarts MA, Brun-Buisson C, Cook DJ et al (2007) Antibiotic management of suspected nosocomial ICU-acquired infection: does prolonged empiric therapy improve Outcome? Intensive Care Med 33:1369-1378

212. Stevens V, Dumyati G, Fine LS et al (2011) Cumulative antibiotic expo sures over time and the risk of Clostridium difficile infection. Clin Infect Dis 53:42-48

213. Goossens H (2009) Antibiotic consumption and link to resistance. Clin Microbiol Infect 15(Suppl 3):12-15

214. PneumA Trial Group, Chastre J, WolffM, Fagon JY et al (2003) Comparison of 8 vs 15 days of antibiotic therapy for ventilator-associated pneu monia in adults: a randomized trial. JAMA 290:2588-2598

215. Choudhury G, Mandal P, Singanayagam A et al (2011) Seven-day antibi otic courses have similar efficacy to prolonged courses in severe community-acquired pneumonia-a propensity-adjusted analysis. Clin Microbiol Infect 17:1852-1858

216. Pugh R, Grant C, Cooke RP, Dempsey G (2015) Shortcourse ver sus prolonged-course antibiotic therapy for hospital-acquired pneumonia in critically ill adults. Cochrane Database Syst Rev. https://doi. org/10.1002/14651858.CD007577.pub3

217. Sawyer RG, Claridge JA, Nathens $A B$ et a (2015) Trial of short-course antimicrobial therapy for intraabdominal infection. $N$ Engl J Med 372:1996-2005

218. Eliakim-Raz N, Yahav D, Paul Metal (2013) Duration of antibiotic treatment for acute pyelonephritis and septic urinary tract infection - 7 days or less versus longer treatment: systematic review and meta-analysis of randomized controlled trials. JAntimicrob Chemother 68:2183-2191

219. Rattan R, Allen CJ, Sawyer RG et al (2016) Patients with complicated intra-abdominal infection presenting with sepsis do not require longer duration of antimicrobial therapy. J Am Coll Surg 222:440-446

220. Hepburn MJ, Dooley DP, Skidmore PJ et al (2004) Comparison of short course ( 5 days) and standard (10 days) treatment for uncomplicated cellulitis. Arch Intern Med 164:1669-1674

221. Chaudhry Zl, Nisar S, Ahmed U, Ali M (2000) Short course of antibiotic treatment in spontaneous bacterial peritonitis: a randomized con trolled study. J Coll Physicians Surg Pak 10(8):284-288

222. Infectious Diseases Society of America, Freifeld AG, Bow EJ, Sepkowitz KA et al (2011) Clinical practice guideline for the use of antimicrobial agents in neutropenic patients with cancer: 2010 update by the infectious diseases society of america. Clin Infect Dis 52:e56-e93

223. Jack L, Bal AM, Harte $S$ et al (2016) International guidelines: the need to standardize the management of candidaemia. Infect Dis 48:779-781

224. American Heart Association Committee on Rheumatic Fever, Endocarditis, and Kawasaki Disease of the Council on Cardiovascular Disease in the Young, Council on Clinical Cardiology, Council on Cardiovascular Surgery and Anesthesia, Stroke Council, Baddour LM, Wilson WR, Bayer AS et al (2015) Infective Endocarditis in Adults: Diagnosis, Antimicrobial Therapy, and Management of Complications: A Scientific Statement for Healthcare Professionals From the American Heart Association. Circulation 132:1435-1486

225. Weiss CH, Moazed F, McEvoy CA et al (2011) Prompting physicians to address a daily checklist and process of care and clinical outcomes: a singlesite study. Am J Respir Crit Care Med 184:680-686

226. PCRAGAStudy Group,SpanishStem Cell Transplantation Group, Study Group of Medical Mycology of the Spanish Society of Clinical Microbiology and Infectious Diseases, Spanish Network for Research in InfectiousDiseases, Aguado JM, Vázquez L, Fernández-Ruiz M et al (2015) Serum galactomannan versus a combination of galacto mannan and 
polymerase chain reaction-based Aspergillus DNA detection for early therapy of invasive aspergillosis in high-risk hematological patients: a randomized controlled trial. Clin Infect Dis 60:405-414

227. Hou TY, Wang SH, Liang SX et al (2015) The screening performance of serum 1,3-beta-DGlucan in patients with invasive fungal diseases: $a$ meta-analysis of prospective cohort studies. PLoS ONE 10:e131602

228. Schuetz P, Briel M, Christ-Crain M et al (2012) Procalcitonin to guide ini tiation and duration of antibiotic treatment in acute respiratory infec tions: an individual patient data meta-analysis. Clin Infect Dis 55:651-662

229. Matthaiou DK, Ntani G, Kontogiorgi $M$ et al (2012) An ESICM systematic review and metaanalysis of procalcitonin-guided antibiotic therapy algorithms in adult critically ill patients. Intensive Care Med 38:940-949

230. Prkno A, Wacker C, Brunkhorst FM et al (2013) Procalcitonin-guided therapy in intensive care unit patients with severe sepsis and sep tic shock-a systematic review and meta-analysis. Crit Care 17:R291

231. Westwood M, Ramaekers B, Whiting P et al (2015) Procalcitonin testing to guide antibiotic therapy for the treatment of sepsis in intensive care settings and for suspected bacterial infection in emergency depart ment settings: a systematic review and cost-effectiveness analysis. Health Technol Assess 19(96):1-236

232. Wacker C, Prkno A, Brunkhorst FM et al (2013) Procalcitonin as a diag nostic marker for sepsis: a systematic review and meta-analysis. Lancet Infect Dis 13:426-435

233. Soni NJ, Samson DJ, Galaydick JL et al (2013) Procalcitonin-guided anti biotic therapy: a systematic review and meta-analysis. JHosp Med 8:530-540

234. de Jong E, van Oers JA, Beishuizen A et al (2016) Efficacy and safety of procalcitonin guidance in reducing the duration of antibiotic treat ment in critically ill patients: a randomised, controlled, open-label trial. Lancet Infect Dis 16:819-827

235. Lesprit P, Landelle C, Girou E et al (2010) Reassessment of intrave nous antibiotic therapy using a reminder or direct counselling.J Antimicrob Chemother 65:789-795

236. Paul M, Dickstein Y, Raz-Pasteur A (2016) Antibiotic de-escalation for bloodstream infections and pneumonia: systematic review and meta-analysis. Clin Microbiol Infect 22:960-967

237. Jimenez MF, Marshall JC, International Sepsis Forum (2001) Source control in the management of sepsis. Intensive Care Med 27(Suppl 1):S49-S62

238. Azuhata T, Kinoshita K, Kawano D et al (2014) Time from admission to initi ation of surgery for source control is a critical determinant of survival in patients with gastrointestinal perforation with associated septic shock. Crit Care 18:R87

239. Bloos F, Thomas-Rüddel D, Rüddel H et al (2014) MEDUSA Study Group: Impact of compliance with infection management guidelines on out come in patients with severe sepsis: a prospective observational multi-center study. Crit Care 18:R42

240. FEAST Trial Group, Maitland K, Kiguli S, Opoka RO et al (2011) Mortality after fluid bolus in African children with severe infection. N Engl J Med 364:2483-2495

241. Acheampong A, Vincent JL (2015) A positive fluid balance is an independent prognostic factor in patients with sepsis. Crit Care 19:251

242. Brotfain E, Koyfman L, Toledano R et al (2016) Positive fluid balance as a major predictor of clinical
Outcome of patients with sepsis/septic shock after ICU discharge. Am JEmerg Med 34:2122-2126

243. Mitchell KH, Carlbom D, Caldwell E et al (2015) Volume overload: prevalence, risk factors, and functional outcome in survivors of septic shock. Ann Am Thorac Soc 12:1837-1844

244. de Oliveira FS, Freitas FG, Ferreira EM et al (2015) Positive fluid balance as a prognostic factor for mortality and acute kidney injury in severe sepsis and septic shock. JCrit Care 30:97-101

245. Malbrain ML, Marik PE, Witters I et al (2014) Fluid overload, de-resuscitation, and Outcomes in critically ill or injured patients: a systematic review with suggestions for clinical practice. Anaesthesio Intensive Ther 46:361-380

246. Yunos NM, Bellomo R, Hegarty C et al (2012) Association between a chloride-liberal vs chloriderestrictive intravenous fluid administra tion strategy and kidney injury in critically ill adults. JAMA 308:1566-1572

247. Yunos NM, Bellomo R, Glassford N, Sutcliffe $H$, Lam Q, BaileyM(2015)Chloride-liberalvs. chloriderestrictive intravenous fluid administration and acutekidneyinjury:anextendedanalysis. Intensive Care Med 41(2):257-264. https://doi.org/10.1007/ s00134-014-3593-0

248. Semler M et al (2018) Balanced crystalloids versus saline in critically ill adults. $N$ Engl J Med 378:829-839

249. Fluids in Sepsis and Septic Shock Group, Rochwerg B, Alhazzani W, Sindi A et al (2014) Fluid resuscitation in sepsis: a systematic review and network meta-analysis. Ann Intern Med 161:347-355

250. SPLIT Investigators, ANZICS CTG, Young P, Bailey M Beasley Retal (2015) Effect of a Buffered Crystalloid Solution vs Saline on Acute Kidney Injury Among Patients in the Intensive Care Unit: The SPLIT Randomized Clinical Trial. JAMA 314:1701-1710

251. Semler MW, Self WH, Wanderer JP, Ehrenfeld JM, Wang L, Byrne DW, Stollings JL, Kumar AB, Hughes CG, Hernandez A, Guillamondegui OD, May AK, Weavind L, Casey JD, Siew ED, Shaw AD, Bernard GR, Rice TW (2018) SMART investigators and the pragmatic critical care research group. Balanced crystalloids versus saline in critically ill adults. NEngl J Med 378(9):829-839

252. Annane D, Siami S, Jaber S et al (2013) Effects of fluid resuscitation with colloids vs crystalloids on mortality in critically ill patients presenting with hypovolemic shock: the CRISTAL randomized trial. JAMA 310:1809-1817

253. Haase N, Perner A, Hennings Ll et al (2013) Hydroxyethyl starch $130 / 0.380 .45$ versus crystalloid or albumin in patients with sepsis: systematic review with meta-analysis and trial sequential analysis. BMJ346:f839

254. Day NP, Phu NH, Bethell DP et al (1996) The effects of dopamine and adrenaline infusions on acidbase balance and systemic haemody namics in severe infection. Lancet 348:219-223

255. De Backer D, Creteur J, Silva E et al (2003) Effects of dopamine, nor epinephrine, and epinephrine on the splanchnic circulation in septic shock: which is best? Crit Care Med 31:1659-1667

256. Martin C, Papazian L, Perrin G et al (1993) Norepinephrine or dopamine for the treatment of hyperdynamic septic shock? Chest 103:1826-1831

257. Martin C, Viviand X, Leone M et al (2000) Effect of norepinephrine on the Outcome of septic shock. Crit Care Med 28:2758-2765

258. Bollaert PE (1990) Chest. https://doi.org/10.1378/ chest.98.4.949

259. Levy B, Bollaert PE, Charpentier C et al (1997) Comparison of norepi nephrine and dobutamine to epinephrine for hemodynamics, lactate metabolism, and gastric tonometric variables in septic shock: a pro spective, randomized study. Intensive Care Med 23:282-287

260. Zhou SX, Qiu HB, Huang YZ et al (2002) Effects of norepinephrine, epi nephrine, and norepinephrine-dobutamine on systemic and gastric mucosal oxygenation in septic shock. Acta Pharmacol Sin 23:654-658

261. Mackenzie SJ, Kapadia F, Nimmo GR et al (1991) Adrenaline in treatment of septic shock: effects on haemodynamics and oxygen transport. Intensive Care Med 17:36-39

262. Moran JL, O'Fathartaigh MS, Peisach ARet al (1993) Epinephrine as an inotropic agent in septic shock: a dose-profile analysis. Crit Care Med 21:70-77

263. Yamazaki T, Shimada Y, Taenaka N et al (1982) Circulatory responses to afterloading with phenylephrine in hyperdynamic sepsis. Crit Care Med 10:432-435

264. Regnier B, Rapin M, Gory G et al (1977) Haemodynamic effects of dopa mine in septic shock. Intensive Care Med 3:47-53

265. Beck GC, Brinkkoetter P, Hanusch C, : et al (2004) Clinical review: immu-nomodulatory effects of dopamine in general inflammation. Crit Care 8(6):485-491

266. Avni T, Lador A, Lev S et al (2015) Vasopressors for the treatment of septic shock: systematic review and meta-analysis. PLoSONE 10:e129305

267. CAT Study investiga tors, Myburgh JA, Higgins A, Jovanovska A et al (2008) A comparison of epinephrine and norepinephrine in critically ill patients. Intensive Care Med 34:2226-2234

268. Patel BM, Chittock DR, Russell JA et al (2002) Beneficial effects of short-term vasopressin infusion during severe septic shock. Anesthesiology 96:576-582

269. Dünser MW, Mayr AJ, Ulmer H et al (2003) Arginine vasopressin in advanced vasodilatory shock: a prospective, randomized, controlled study. Circulation 107:2313-2319

270. Lauzier F, Lévy B, Lamarre Petal (2006) Vasopressin or norepinephrine in early hyperdynamic septic shock: a randomized clinical trial. Intensive Care Med 32:1782-1789

271. Holmes CL, Walley KR, Chittock DR et al (2001) The effects of vasopres sin on hemodynamics and renal function in severe septic shock: a case series. Intensive Care Med 27:1416-1421

272. Malay MB, Ashton RCJr,Landry DWetal (1999) Lowdose vasopres sin in the treatment of vasodilatory septic shock. J Trauma 47:699-703 (discussion 703)

273. O'Brien A, Clapp L, Singer M (2002) Terlipressin for norepinephrine-resis tant septic shock. Lancet 359:1209-1210

274. Sharshar T, Blanchard A, Paillard M et al (2003) Circulating vasopressin levels in septic shock. Crit Care Med 31:1752-1758

275. VASST Investigators, Russell JA, Walley KR, Singer J et al (2008) Vasopressin versus norepinephrine infusion in patients with septic shock. N Engl J Med 358:877-887

276. VANISH Investigators, Gordon AC, Mason AJ, Thirunavukkarasu N et al (2016) Effect of early vasopressin vs norepinephrine on kidney failure in patients with septic shock: the VANISH randomized clinical trial. JAMA 316:509-518

277. Albanèse J, Leone $M$, Delmas A et al (2005) Terlipressin or norepinephrine in hyperdynamic septic shock: a prospective, randomized study. Crit Care Med 33:1897-1902 
278. Morelli A, Ertmer C, Lange M et al (2008) Effects of short-term simultane ous infusion of dobutamine and terlipressin in patients with septic shock: the DOBUPRESS study. Br J Anaesth 100:494-503

279. Morelli A, Ertmer C, Rehberg S et al (2009) Continuous terlipressin ver sus vasopressin infusion in septic shock (TERLIVAP): a randomized, controlled pilot study. Crit Care 13:R130

280. Zhou F, Mao Z, Zeng X et al (2015) Vasopressors in septic shock: a sys tematic review and network meta-analysis. Ther Clin Risk Manag 11:1047-1059

281. De Backer D, Aldecoa C, Njimi H et al (2012) Dopamineversus norepinephrine in the treatment of septic shock: a meta-analysis. Crit Care Med 40:725-730

282. De Backer D (2010) N Engl J Med. https://doi.org/ 10.1056/NEJMoa0907118

283. Gattinoni L, Brazzi L, Pelosi P et al (1995) A trial of goal-oriented hemodynamic therapy in critically ill patients. Sv02 Collaborative Group. N Engl J Med 333:1025-1032

284. Hayes MA, Timmins AC, Yau EH et al (1994) Elevation of systemic oxygen delivery in the treatment of critically ill patients. N Engl J Med 330:1717-1722

285. Hollenberg SM, Ahrens TS, Annane D et al (2004) Practice parameters for hemodynamic support of sepsis in adult patients: 2004 update. Crit Care Med 32:1928-1948

286. CATS Study Group, Annane D, Vignon P, Renault A et al (2007) Norepinephrine plus dobutamine versus epinephrine alone for manage ment of septic shock: a randomised trial. Lancet 370:676-684

287. ProCess Investigators, Yealy DM, Kellum JA et al (2014) A randomized trial of protocolbased care for early septic shock. N Engl J Med 370(18):1683-1693

288. ARISE Investigators, Anzics Clinical Trials Group, Peake SL, : et al (2014) Goal-directed resuscitation for patients with early septic shock. N Engl J Med 371(16):1496-1506

289. Cohn JN (1967) Blood pressure measurement in shock. Mechanism of inaccuracy in ausculatory and palpatory methods. JAMA 199:118-122

290. Hollenberg SM, Parrillo JE (1997) Shock. In: Braunwald E, Isselbacher KJ, Wilson JD et al (Hrsg) Harrison's principles of internal medicine, 14. Aufl. McGraw-Hill, New York, S214-222

291. Scheer B, Perel A, Pfeiffer UJ (2002) Clinical review: complications and riskfactors of peripheral arterial catheters used for haemodynamic monitoring in anaesthesia and intensive care medicine. Crit Care 6:199-204

292. Gu WJ, Wu XD, Wang F et al (2016) Ultrasound guidance facilitates radial artery catheterization: a meta-analysis with trial sequential analysis of randomized controlled trials. Chest 149:166-179

293. O'Horo JC, Maki DG, Krupp AE et al (2014) Arterial catheters as a source of bloodstream infection: a systematic review and meta-analysis. Crit Care Med 42:1334-1339

294. Barton P, Garcia J, Kouatli A et al (1996) Hemodynamic effects of i.v. mil rinone lactate in pediatric patients with septic shock. A prospective, double-blinded, randomized, placebo-controlled, interventional study. Chest 109:1302-1312

295. Morelli A, Teboul JL, Maggiore SM et al (2006) Effects of levosimendan on right ventricular afterload in patients with acute respiratory distress syndrome: a pilot study. Crit Care Med 34:2287-2293

296. Morelli A, De Castro S, Teboul JL et al (2005) Effects of levosimendan on systemic and regional hemodynamics in septic myocardial depres sion. Intensive Care Med 31:638-644

297. Gordon AC, Perkins GD, Singer M et al (2016) Levosimendan for the pre vention of acute organ dysfunction in sepsis. N Engl J Med. https://doi. org/10.1056/nejmoa1609409

298. Schmittinger CA, Torgersen C, Luckner Getal (2012) Adverse cardiac events during catecholamine vasopressor therapy: a prospective observational study. Intensive Care Med 38(6):950-958

299. Leibovici Let al (2007) QJM 100:629-634

300. Pemberton P, Veenith T, Snelson C, Whitehouse T (2015) Is it time to beta block the septic patient? Biomed Res Int 2015:424308

301. Lechat P, Packer M, Chalon S et al (1998) Clinical effects of beta-adrenergic blockade in chronic heart failure: a meta-analysis of double-blind, placebo-controlled, randomized trials. Circulation 98(12):1184-1191

302. Martin N, Manoharan K, Thomas Jet al (2018) Betablockers and inhibitors of the renin-angiotensin aldosterone system for chronic heart failure with preserved ejection fraction. Cochrane Database Syst Rev. https://doi.org/10.1002/14651858. CD012721.pub2

303. Garnock-Jones KP (2012) Esmolol: a review of its use in the short-term treatment of tachyarrhythmias and the short-term control of tachycardia and hypertension. Drugs 72(1):109-132

304. Sanfilippo F, Santonocito C, Morelli A, Foex P (2015) Beta-blocker use in severe sepsis and septic shock: a systematic review. Curr Med Res Opin 31(10):1817-1825

305. Morelli A, Ertmer C, Westphal M et al (2013) Effect of heart rate control with esmolol on hemodynamic and clinical Outcomes in patients with septic shock: a randomized clinical trial. JAMA 310(16):1683-1691

306. Annane D, Bellissant E, Bollaert PE et al (2009) Corticosteroids in the treatment of severe sepsis and septic shock in adults: a systematic review. JAMA 301:2362-2375

307. Bollaert PE, Charpentier C, Levy B et al (1998) Reversal of late septic shock with supraphysiologic doses of hydrocortisone. Crit Care Med 26:645-650

308. Briegel J, Forst H, Haller M et al (1999) Stress doses of hydrocortisone reverse hyperdynamic septic shock: a prospective, randomized, double-blind, single-center study. Crit Care Med 27:723-732

309. CORTICUS Study Group, Sprung CL, Annane D, Keh D et al (2008) Hydrocortisone therapy for patients with septic shock. N Engl J Med 358:111-124

310. Sligl WI, Milner DA Jr, Sundar S et al (2009) Safety and efficacy of cortico steroids for the treatment of septic shock: a systematic review and metaanalysis. Clin Infect Dis 49:93-101

311. Volbeda M, Wetterslev J, Gluud C et al (2015) Glucocorticosteroids for sepsis: systematic review with meta-analysis and trial sequential analysis. Intensive Care Med 41:1220-1234

312. AnnaneD, SébilleV, CharpentierCetal (2002) Effect of treatment with low doses of hydrocortisone and fludrocortisone on mortality in patients with septic shock. JAMA 288:862-871

313. CORTICUS Study Group, Briegel J, Sprung CL, Annane D et al (2009) Multicenter comparison of cortisol as measured by different meth ods in samples of patients with septic shock. Intensive Care Med 35:2151-2156

314. Allolio B, Dörr H, Stuttmann R et al (1985) Effect of a single bolus of etomi date upon eight major corticosteroid hormones and plasma ACTH. Clin Endocrinol 22:281-286
315. KETASED Collaborative Study Group, Jabre $P$, Combes X, Lapostolle F et al (2009) Etomidate versus ketamine for rapid sequence intuba tion in acutely ill patients: a multicentre randomised controlled trial. Lancet 374:293-300

316. Oppert M, Schindler R, Husung C et al (2005) Lowdose hydrocortisone improves shock reversal and reduces cytokine levels in early hyper dynamic septic shock. Crit Care Med 33:2457-2464

317. Yildiz O, Doganay M, Aygen B et al (2002) Physiological-dose steroid therapy in sepsis [ISRCTN36253388]. Crit Care 6:251-259

318. Keh D, Boehnke T, Weber-Cartens S et al (2003) Immunologic and hemo dynamic effects of "lowdose" hydrocortisone in septic shock: a doubleblind, randomized, placebo-controlled, crossover study. Am J Respir Crit Care Med 167:512-520

319. Huh JW, Choi HS, Lim CM et al (2011) Low-dose hydrocortisone treat ment for patients with septic shock: a pilot study comparing 3 days with 7 days. Respirology 16:1088-1095

320. SepNet-Critical Care Trials Group, Keh D, Trips E, Marx $\mathrm{G}$ et al (2016) Effect of hydrocortisone on development of shock among patients with severe sepsis: the HYPRESS randomized clinical trial.JAMA 316:1775-1785

321. Weber-Carstens $S$, Deja $M$, Bercker $S$ et al (2007) Impact of bolus appli cation of low-dose hydrocortisone on glycemic control in septic shock patients. Intensive Care Med 33:730-733

322. Holst LB, Haase N, Wetterslev J et al (2014) Lower versus higher hemoglobin threshold for transfusion in septic shock. N Engl J Med 371:1381-1391

323. Rygard SL, Holst LB, Wetterslev J et al (2016) Long-term Outcomes in patients with septic shock transfused at a lower versus a higher haemoglobin threshold: the TRISS randomised, multicentre clinical trial. Intensive Care Med 42:1685-1694

324. Dupuis C, Sonneville R, Adrie Cet al (2017) Impact of transfusion on patients with sepsis admitted in intensive care unit: a systematic review and metaanalysis. Ann Intensive Care 7:5

325. Dupuis C, Garrouste-Orgeas M, Bailly $S$ et al (2017) Effect of transfusion on mortality and other adverse events among critically ill septic patients: an observational study using a marginal structural cox model. Crit Care Med 45:1972-1980

326. Investigators P, Rowan KM, Angus DC et al (2017) Early, goal-directed therapy for septic shock-a patient-level meta-analysis. N Engl J Med 376:2223-2234

327. Bundesärztekammer (2014) Querschnitts-Leitlinien zur Therapie mit Blutkomponenten und Plasmaderivaten - Herausgegeben von der Bundesärztekammer auf Empfehlung ihres Wissenschaftlichen Beirats, 4. Aufl. Deutscher ÄrzteVerlag, Köln

328. Corwin HL, Gettinger A, Rodriguez RM et al (1999) Efficacy of recombinant human erythropoietin in the critically ill patient: a randomized, doubleblind, placebo-controlled trial. Crit Care Med 27:2346-2350

329. Corwin HL, Gettinger A, Pearl RG et al (2002) Efficacy of recombinant human erythropoietin in critically ill patients: a randomized controlled trial. JAMA 288:2827-2835

330. Score-Based Immunoglobulin therapy of Sepsis (SBITS) Study Group, Werdan K, Pilz G, Bujdoso O et al (2007) Score-based immunoglobulin G therapy of patients with sepsis: the SBITS study. Crit Care Med 35(200):2693-2701

331. Alejandria MM, Lansang MA, Dans LF, Mantaring JB 3rd (2013) Intravenous immunoglobulin for 
treating sepsis, severe sepsis and septic shock. Cochrane Database Syst Rev. https://doi.org/10. 1002/14651858.CD001090.pub2

332. Laupland KB, Kirkpatrick AW, Delaney A (2007) Polyclonal intravenous immunoglobulin for the treatment of severe sepsis and septic shock in critically adults: a systematic review and metaanalysis. Crit Care Med 35:2686-2692

333. Pildal J, Gotzsche PC (2004) Polyclonal immunoglobulin for treatment of bacterial sepsis: a systematic review. Clin Infect Dis 39:38

334. Kreymann KG, de Heer G, Nierhaus A et al (2007) Use of polyclonal immunoglobulins as adjunctive therapy for sepsis or septic shock. Crit Care Med 35:2677-2685

335. Turgeon AF, Hutton B, Fergusson DA et al (2007) Meta-analysis: intravenous immunoglobulin in critically ill adult patients with sepsis. Ann Intern Med 146:193-203

336. Shankar-Hari M, Culshaw N, Post B et al (2015) Endogenous IgG hypogammaglobulinaemia in critically ill adults with sepsis: systematic review and meta-analysis. Intensive Care Med 41:1393-1401

337. Welte T, Dellinger RP, Ebelt $\mathrm{H}$ et al (2018) Efficacy and safety of trimodulin, a novel polyclonal antibody preparation, in patients with severe community-acquired pneumonia: a randomized, placebo-controlled, double-blind, multicenter, phase II trial (CIGMA study). Intensive Care Med 44(4):438-448

338. Zhou F, Peng Z, Murugan R et al (2013) Blood purification and mortality in sepsis:a meta-analysis of randomized trials. Crit Care Med 41:2209-2220

339. ABDOMIX Group, Payen DM, Guilhot J, Launey Y etal (2015) Earlyuse of polymyxin Bhemoperfusion in patients with septic shock due to peri tonitis: a multicenter randomized control trial. Intensive Care Med 41:975-984

340. Klein DJ, Foster D, Schorr CA et al (2014) The EUPHRATES trial (Evaluating the Use of Polymyxin B Hemoperfusion in a Randomized controlled trial of Adults Treated for Endotoxemia and Septic shock): study protocol for a randomized controlled trial. Trials 15:218

341. EUPHRATES Trial Investigators, Dellinger RP, Bagshaw SM, Antonelli M et al (2018) Effect of targeted polymyxin $B$ hemoperfusion on 28-day mortality in patients with septic shock and elevated endotoxin level: the EUPHRATES randomized clinical trial.JAMA320(14):1455-1463

342. LivigniS, BertoliniG, RossiC, al GiViT,GiViTI:Gruppo Italiano per la Valutazione degli Interventi in Terapia Intensiva (Italian Group for the Evaluation of Interventions in Intensive (are Medicine) is an independent collaboration network of Italian Intensive Care units (2014) Efficacy of coupled plasma filtration adsorption (CPFA) in patients with septic shock: a multi center randomised controlled clinical trial. BmjOpen 4:e3536

343. Schädler D, Pausch C, Heise D et al (2017) The effect of a novel extracorporeal cytokine hemoadsorption Device on IL-6 elimination in septic patients: A randomized controlled trial.PLoS ONE 12(10):e187015

344. Hawchar F, László I, Öveges N et al (2019) Extracorporeal cytokine adsorption in septic shock: a proof of concept randomized, controlled pilot study. J Crit Care 49:172-178

345. Allingstrup $M$, Wetterslev J, Ravn FB et al (2016) Antithrombin III for criti cally ill patients. Cochrane Database Syst Rev. https://doi.org/10. 1002/14651858.CD005370.pub3
346. Warren BL, Eid A, Singer P et al (2001) Kybersept trial study group: caring for the critically ill patient. High-dose antithrombin III in severe sepsis: a randomized controlled trial. JAMA 286:1869-1878

347. Vincent JL, Ramesh MK, Ernest D et al (2013) A randomized, double-blind, placebo-controlled, Phase $2 b$ study to evaluate the safety and efficacy of recombinant human soluble thrombomodulin, ART-123, in patients with sepsis and suspected disseminated intravascular coagulation. Crit Care Med 41:2069-2079

348. Yamakawa K, Ogura H, Fujimi S et al (2013) Recombinant human soluble thrombomodulin in sepsis-induced disseminated intravascular coag ulation: a multicenter propensity score analysis. Intensive Care Med 39:644-652

349. Canadian Critical Care Trials Group, Zarychanski R, Abou-Setta AM, Kanji S et al (2015) The efficacy and safety of heparin in patients with sepsis: a systematic review and metaanalysis. Crit Care Med 43:511-518

350. Putensen C, Theuerkauf $N$, Zinserling J et al (2009) Meta-analysis: ventilation strategies and outcomes of the acute respiratory distress syndrome and acute lung injury. Ann Intern Med 151(8):566-576. https://doi.org/10.7326/00034819-151-8-200910200-00011

351. Petrucci N, de Feo C (2013) Lung protective ventilation strategy for the acute respiratory distress syndrome. Cochrane Database Syst Rev. https://doi.org/10.1002/14651858.CD003844. pub4

352. The Acute Respiratory Distress Syndrome Network (2000) Ventilation with lower tidal volumes as compared with traditional tidal volumes for acute lung injury and the acute respiratory distress syndrome. N Engl J Med 342(18):1301-1308. https://doi.org/10.1056/NEJM200005043421801

353. Amato MB, Barbas CS, Medeiros DM et al (1998) Effect of a protective-ventilation strategy on mortality in the acute respiratory distress syndrome. N Engl J Med 338:347-354. https://doi. org/10.1056/NEJM199802053380602

354. Brochard L, Roudot-Thoraval F, Roupie E et al (1998) Tidal volume reduction for prevention of ventilator-induced lung injury in acute respiratory distress syndrome. Am J Respir Crit Care Med 158(6):1831-1838. https://doi.org/10.1164/ ajrccm.158.6.9801044

355. Feihl F, Eckert P, Brimioulle S et al (2000) Permissive hypercapnia impairs pulmonary gas exchange in the acute respiratory distress syndrome. Am J Respir Crit Care Med 162(1):209-215. https://doi. org/10.1164/ajrccm.162.1.9907119

356. PinhuL,WhiteheadT,EvansTetal(2003)Ventilatorassociated lung injury. Lancet 361(9354):332-340. https://doi.org/10.1016/S0140-6736(03)12329-X

357. Serpa Neto A, Simonis FD, Barbas CSV et al (2014) Association between tidal volume size, duration of ventilation, and sedation needs in patients without acute respiratory distress syndrome: an individual patient data meta-analysis. Intensive Care Med 40(7):950-957. https://doi.org/10.1007/s00134014-3318-4

358. Serpa Neto A, Cardoso SO, Manetta JA et al (2012) Association between use of lungprotective ventilation with lower tidal volumes and clinical Outcomes among patients without acute respiratory distress syndrome: a metaanalysis. JAMA 308(16):1651-1659. https://doi. org/10.1001/jama.2012.13730

359. Serpa Neto A, Hemmes SNT, Barbas CSV et al (2015) Protective versus conventional ventilation for surgery: a systematic review and individual patient data meta-analysis. Anesthesiology 123(1):66-78 https://doi.org/10.1097/ALN.0000000000000706

360. Stewart TE, Meade MO, Cook DJ et al (1998) Evaluation of a ventilation strategy to prevent barotrauma in patients at high risk for acute respiratory distress syndrome. Pressure- and Volume-Limited Ventilation Strategy Group. NEng JMed 338(6):355-361

361. Brower RG, Shanholtz CB, Fessler HE et al (1999) Prospective, randomized, controlled clinical trial comparing traditional versus reduced tidal volume ventilation in acute respiratory distress syndrome patients. Crit Care Med 27(8):1492-1498

362. Villar J, Kacmarek RM, Perez-Mendez L et al (2006) A high positive end-expiratory pressure, low tidal volume ventilatory strategy improves Outcome in persistent acute respiratory distress syndrome: a randomized, controlled trial. Crit Care Med 34(5):1311-1318

363. Amato MBP, Meade MO, Slutsky AS et al (2015) Driving pressure and survival in the acute respiratory distress syndrome. N Engl J Med 372(8):747-755. https://doi.org/10.1056/NEJMsa1410639

364. Laffey JG, Bellani G, Pham T et al (2016) Potentially modifiable factors contributing to Outcome from acute respiratory distress syndrome: the LUNG SAFE study. Intensive Care Med 42(12):1865-1876. https://doi.org/10.1007/s00134-016-4571-5

365. Sahetya SK, Brower RG (2016) The promises and problems of transpulmonary pressure measurements in acute respiratory distress syndrome. Curr Opin Crit Care 22(1):7-13. https://doi.org/10. 1097/MCC.0000000000000268

366. Extracorporeal Life Support Organization General Guidelines for all ECLS Cases, https://www. elso.org/Portals/0/IGD/Archive/FileManager/ 929122ae88cusersshyerdocumentselsoguidelines generalalleclsversion1.3.pdf

367. Serpa Neto A, Schmidt M, Azevedo LCP et al (2016) Associations between ventilator settings during extracorporeal membrane oxygenation for refractory hypoxemia and Outcome in patients with acute respiratory distress syndrome: a pooled individual patient data analysis. Mechanical ventilation during ECMO. Intensive Care Med 42(11):1672-1684. https://doi.org/10.1007/ s00134-016-4507-0

368. Dellinger RP, Levy MM, Rhodes A et al (2013) Surviving Sepsis Campaign. international guidelines for management of severe sepsis and septic shock, 2012. Intensive Care Med 39(2):165-228. https:// doi.org/10.1007/s00134-012-2769-8

369. Valente Barbas CS, Ísola AM, Farias, de Carvalho AM et al (2014) Brazilian recommendations of mechanical ventilation 2013. Part 2. J Bras Pneumol 40(5):458-486. https://doi.org/10.1590/ S1806-37132014000500003

370. National Guideline Clearinghouse (2009) Care of the patient with aneurysmal subarachnoid hemorrhage. http://www.guideline.gov/ content.aspx?id=34442\&search=ventilation + or+mechanical+ventilation+or+respiratory+failure

371. ÖGARI Leitlinien zur invasiven Beatmung von Intensivpatienten, https://www.oegari.at/web_ files/dateiarchiv/editor/leitlinien_invasiven_ beatmung_aktualisert_2017.pdf

372. Hata JS, Togashi K, Kumar AB et al (2014) The effect of the pressure-volume curve for positive end-expiratory pressure titration on clinical Outcomes in acute respiratory distress syndrome. a systematic review. J Intensive Care Med 29(6):348-356. https://doi.org/10.1177/ 0885066613488747 
373. Cruz SR, Rojas Jl, Nervi R et al (2013) High versus low positive end-expiratory pressure (PEEP) levels for mechanically ventilated adult patients with acute lung injury and acute respiratory distress syndrome. Cochrane Database Syst Rev. https:// doi.org/10.1002/14651858.CD009098.pub2

374. Dasenbrook EC, Needham DM, Brower RG et al (2011) Higher peep in patients with acute lung injury. A systematic review and meta-analysis. Respir Care 56(5):568-575

375. Briel M, Meade M, Mercat A et al (2010) Higher vs lower positive end-expiratory pressure in patients with acute lung injury and acute respiratory distress syndrome. systematic review and metaanalysis. JAMA 303(9):865-873. https://doi.org/ 10.1001/jama.2010.218

376. Phoenix SI, Paravastu S, Columb M et al (2009) Does a higher positive end expiratory pressure decrease mortality in acute respiratory distress syndrome? A systematic review and meta-analysis. Anesthesiology 110(5):1098-1105. https://doi. org/10.1097/ALN.0b013e31819fae06

377. Oba Y, Thameem DM, Zaza T (2009) High levels of PEEP may improve survival in acute respiratory distress syndrome. A meta-analysis. Respir Med 103(8):1174-1181. https://doi.org/10. 1016/j.rmed.2009.02.008

378. Ranieri VM, Suter PM, Tortorella C et al (1999) Effect of mechanical ventilation on inflammatory mediators in patients with acute respiratory distress syndrome: a randomized controlled trial. JAMA 282(1):54-61

379. Meade MO, Cook DJ, Guyatt GH et al (2008) Ventilation strategy using low tidal volumes, recruitment maneuvers, and high positive endexpiratory pressure for acute lung injury and acute respiratory distress syndrome. a randomized controlled trial. JAMA 299(6):637-645. https://doi. org/10.1001/jama.299.6.637

380. Brower RG, Lanken PN, MacIntyre N et al (2004) Higher versus lower positive end-expiratory pressures in patients with the acute respiratory distress syndrome. N Engl J Med 351(4):327-336. https://doi.org/10.1056/NEJMoa032193

381. Huh JW, Jung H, Choi HS et al (2009) Efficacy of positive end-expiratory pressure titration after the alveolar recruitment manoeuvre in patients with acute respiratory distress syndrome. Crit Care 13(1):R22. https://doi.org/10.1186/cc7725

382. Mercat A, J-CM R, Vielle B et al (2008) Positive end-expiratory pressure setting in adults with acute lung injury and acute respiratory distress syndrome. a randomized controlled trial. JAMA 299(6):646-655. https://doi.org/10.1001/jama. 299.6.646

383. Castro-Avila AC, Serón P, Fan E et al (2015) Effect of early rehabilitation during intensive care unit stay on functional status: systematic review and metaanalysis. PLoS ONE 10(7):e130722.https://doi.org/ 10.1371/journal.pone.0130722

384. Lowe GJ, Ferguson ND (2006) Lung-protective ventilation in neurosurgical patients. Curr Opin Crit Care 12(1):3-7

385. Suzumura EA, Figueiro $M$, Normilio-Silva $K$ et al (2014) Effects of alveolar recruitment maneuvers on clinical Outcomes in patients with acute respiratory distress syndrome: a systematic review and meta-analysis. Intensive Care Med 40(9):1227-1240

386. Cavalcanti AB etal (2017) Effect oflung recruitment and titrated positive end-expiratory pressure (PEEP) vs low PEEP on mortality in patients with acute respiratory distress syndrome: a randomized clinical trial. JAMA 318:1335
387. Maggiore SM, Lellouche F, Pigeot J et al (2003) Prevention of endotracheal suctioning-induced alveolar derecruitment in acute lung injury. Am J RespirCrit Care Med 167(9):1215-1224

388. Dyhr T, Bonde J, Larsson A (2003) Lung recruitment manoeuvres are effective in regaining lung volume and oxygenation after open endotracheal suctioning in acute respiratory distress syndrome. CritCare 7(1):55-62

389. S2e-Leitlinie: „Lagerungstherapie und Frühmobilisation zur Prophylaxe oder Therapie von pulmonalen Funktionsstörungen", https://www. awmf.org/uploads/tx_szleitlinien/001-015I_S2e Lagerungstherapie_Fr\%C3\%BChmobilisation pulmonale_Funktionsst\%C3\%B6rungen_201505.pdf

390. Bein T, Bischoff M, Bruckner U et al (2015) Kurzversion S2e-Leitlinie - „Lagerungstherapie und Fruhmobilisation zur Prophylaxe oder Therapie von pulmonalen Funktionsstorungen" (Short version S2e guidelines: "Positioning therapy and early mobilization for prophylaxis or therapy of pulmonary function disorders"). Anaesthesist 64(8):596-611. https://doi.org/10.1007/s00101015-0060-4

391. Bloomfield R, Noble DW, Sudlow A (2015) Prone position for acute respiratory failure in adults. Cochrane Database Syst Rev. https://doi.org/10. 1002/14651858.CD008095.pub2

392. Park SY, Kim HJ, Yoo KH et al (2015) The efficacy and safety of prone positioning in adults patients with acute respiratory distress syndrome: a metaanalysis of randomized controlled trials. J Thorac Dis 7(3):356-367. https://doi.org/10.3978/j.issn. 2072-1439.2014.12.49

393. Davidson JE (2013) Family presence on rounds in neonatal, pediatric, and adult intensive care units. Ann Am Thorac Soc 10:152-156

394. Guérin C, Reignier J, Richard JC et al (2013) PROSEVA study group: prone positioning in severe acute respiratory distress syndrome. N Engl J Med 368:2159-2168

395. La de Oliva P, Schuffelmann C, Gomez-Zamora A et al (2012) Asynchrony, neural drive, ventilatory variability and COMFORT: NAVA versus pressure support in pediatric patients. A non-randomized cross-over trial. Intensive Care Med 38(5):838-846 https://doi.org/10.1007/s00134-012-2535-y

396. Weig T, Janitza S, Zoller M et al (2014) Influence of abdominal obesity on multiorgan dysfunction and mortality in acute respiratory distress syndrome patients treated with prone positioning. J Crit Care 29(4):557-561. https://doi.org/10.1016/j.jcrc. 2014.02.010

397. Maitra S, Bhattacharjee S, Khanna P et al (2014) High-frequency ventilation does not provide mortality benefit in comparison with conventional lung-protective ventilation in acute respiratory distress syndrome. A meta-analysis of the randomized controlled trials. Anesthesiology. https://doi.org/10.1097/ALN.0000000000000306

398. Sud S, Sud M, Friedrich JO et al (2013) Highfrequency ventilation versus conventional ventilation for treatment of acute lung injury and acute respiratory distress syndrome. Cochrane Database Syst Rev. https://doi.org/10.1002/14651858. CD004085.pub3

399. Young D, Lamb SE, Shah S et al (2013) Highfrequency oscillation for acute respiratory distress syndrome. N Engl J Med 368(9):806-813. https:// doi.org/10.1056/NEJMoa1215716

400. Ferguson ND, CookDJ, Guyatt GH et al (2013) Highfrequency oscillation in early acute respiratory distress syndrome. N Engl J Med 368(9):795-805. https://doi.org/10.1056/NEJMoa1215554

401. Papazian L, Forel J-M, Gacouin A et al (2010) Neuromuscular blockers in early acute respiratory distress syndrome. N Engl J Med 363(12):1107-1116. https://doi.org/10.1056/NEJMoa1005372

402. Baron R, Binder A, Biniek Retal (2015) Evidence and consensus based guideline for the management of delirium, analgesia, and sedation in intensive care medicine. Revision 2015 (DAS-Guideline 2015)—short version. Ger Med Sci 13:Doc19. https://doi.org/10.3205/000223

403. Horner D, Cairns C (2011) Early neuromuscular blockade in severe ARDS. J Intensive Care Soc 12(2):153-154. https://doi.org/10.1177/ 175114371101200216

404. Putensen C, Zech S, Wrigge $H$ et al (2001) Longterm effects of spontaneous breathing during ventilatory support in patients with acute lung injury. Am J Respir Crit Care Med 164:43-49

405. Freebairn R (2004) How relaxed should we be with acute respiratory distress syndrome? Crit Care Med 32(1):296-298. https://doi.org/10.1097/01.CCM. 0000104925.62805.D9

406. Levine S, Nguyen T, Taylor N et al (2008) Rapid disuse atrophy of diaphragm fibers in mechanically ventilated humans. N Engl J Med 358(13):1327-1335. https://doi.org/10.1056/ NEJMoa070447

407. Kollisch-Singule M, Emr B, Smith B et al (2014) Airway pressure release ventilation reduces conducting airway micro-strain in lung injury. J Am Coll Surg 219(5):968-976. https://doi.org/10. 1016/j.jamcollsurg.2014.09.011

408. Zhou Y et al (2017) Early application of airway pressure release ventilation may reduce the duration of mechanical ventilation in acute respiratory distress syndrome. Intensive Care Med 43:1648

409. Kiehl M, Schiele C, Stenzinger W et al (1996) Volume-controlled versus biphasic positive airway pressure ventilation in leukopenic patients with severe respiratory failure. Crit Care Med 24:780-784

410. Kaplan LJ, Bailey H, Formosa V (2001) Airway pressure release ventilation increases cardiac performance in patients with acute lung injury/ adult respiratory distress syndrome. Crit Care 5(4):221-226

411. Gonzalez M, Arroliga AC, Frutos-Vivar F et al (2010) Airway pressure release ventilation versus assistcontrol ventilation: a comparative propensity score and international cohort study. Intensive Care Med 36:817-827

412. Singh B, Tiwari AK, Singh K et al (2014) beta2 agonist for the treatment of acute lung injury: a systematic review and meta-analysis. Respir Care 59(2):288-296. https://doi.org/10.4187/respcare. 02571

413. Sakr Y, Vincent JL, Reinhart Ket al (2005) High tidal volume and positive fluid balance are associated with worse Outcome in acute lung injury. Chest 128(5):3098-3108

414. Bundesärztekammer, Kassenärztliche Bundesvereinigung, Arbeitsgemeinschaft der Wissenschaftlichen Medizinischen Fachgesellschaften (2012) Nationale VersorgungsLeitlinie COPD. http://www.awmf.org/uploads/tx_szleitlinien/ nvl-003I_S3_COPD_abgelaufen.pdf

415. Blackwood B, Burns KE, Cardwell CR et al (2014) Protocolized versus non-protocolized weaning for reducing the duration of mechanical ventilation in critically ill adult patients. Cochrane Database 
Syst Rev. https://doi.org/10.1002/14651858. CD006904.pub3

416. Blackwood B, Murray M, Chisakuta A et al (2013) Protocolized versus non-protocolized weaning for reducing the duration of invasive mechanical ventilation in critically ill paediatric patients. Cochrane Database Syst Rev. https://doi.org/10. 1002/14651858.CD009082.pub2

417. Blackwood B, Alderdice F, Burns K et al (2011) Use of weaning protocols for reducing duration of mechanical ventilation in critically ill adult patients Cochrane systematic review and meta-analysis 342. BMJ.https://doi.org/10.1136/bmj.c7237

418. Schönhofer B, Geiseler J, Dellweg Det al (2014) Prolongiertes Weaning. S2k-Leitlinie herausgegeben von der Deutschen Gesellschaftfür Pneumologie und Beatmungsmedizin e.V (Prolonged weaning: S2k-guideline published by the German Respiratory Society). Pneumologie 68(1):19-75. https://doi. org/10.1055/s-0033-1359038

419. Lung Open Ventilation Study Investigators, Meade MO, Cook DJ, Guyatt GH et al (2008) Ventilation strategy using low tidal volumes, recruitment maneuvers, and high positive endexpiratory pressure for acute lung injury and acute respiratory distress syndrome: a randomized controlled trial. JAMA 299:637-645

420. Expiratory Pressure (Express) Study Group, Mercat A, Richard JC, Vielle B et al (2008) Positive end-expiratory pressure setting in adults with acute lung injury and acute respiratory distress syndrome: a randomized controlled trial. JAMA 299:646-655

421. Ferrer $M$, Esquinas $A$, Leon $M$ et al (2003) Noninvasive ventilation in severe hypoxemic respiratory failure: a randomized clinical trial. Am J Respir Crit Care Med 168:1438-1444

422. Antonelli M, Conti G, Rocco M et al (1998) A comparison of noninvasive positive-pressure ventilation and conventional mechanical ventilation in patients with acute respiratory failure. $\mathrm{N}$ Engl J Med 339:429-435

423. FLORALI Study Group, REVA Network, Frat JP, Thille AW, Mercat A et al (2015) High-flow oxygen through nasal cannula in acute hypoxemic respiratory failure. N Engl J Med 372:2185-2196

424. Ely EW, Baker AM, Dunagan DP et al (1996) Effect on the duration of mechanical ventilation of identifying patients capable of breathing spontaneously. N Engl J Med 335:1864-1869

425. Kress JP, Pohlman AS, O'Connor MF et al (2000) Daily interruption of sedative infusions in critically ill patients undergoing mechanical ventilation. NEngl J Med 342:1471-1477

426. GirardTD, Kress JP, FuchsBDetal (2008) Efficacyand safety of a paired sedation and ventilator weaning protocol for mechanically ventilated patients in intensive care (Awakening and Breathing Controlled trial): a randomised controlled trial. Lancet 371:126-134

427. Schweickert WD, Pohlman MC, Pohlman AS et al (2009) Early physical and occupational therapy in mechanically ventilated, critically ill patients: a randomised controlled trial. Lancet 373:1874-1882

428. Sedation Practice in Intensive Care Evaluation (SPICE) Study Investigators, ANZICS Clinical Trials Group, Shehabi Y, Bellomo R, Reade MC et al (2012) Early intensive care sedation predicts long term mortality in ventilated critically ill patients. Am J Respir Crit Care Med 186:724-731

429. Brook AD, Ahrens TS, Schaiff R et al (1999) Effect of a nursing-imple mented sedation protocol on the duration of mechanical ventilation. Crit Care Med 27:2609-2615

430. Bucknall TK, Manias E, Presneill JJ (2008) A randomized trial of protocol directed sedation management for mechanical ventilation in an Australian intensive care unit. Crit Care Med 36:1444-1450

431. Kollef MH, Levy NT, Ahrens TS et al (1998) The use of continuous i.v. seda tion is associated with prolongation of mechanical ventilation. Chest 114:541-548

432. Carson SS, Kress JP, Rodgers JE et al (2006) A randomized trial of inter mittent lorazepam versus propofol with daily interruption in mechani cally ventilated patients. Crit Care Med 34:1326-1332

433. SLEAP Investigators, Canadian Critical Care Trials Group, Mehta S, Burry L, Cook D et al (2012) Daily sedation interruption in mechani cally ventilated critically ill patients cared for with a sedation proto col: a randomized controlled trial. JAMA 308:1985-1992

434. Jansen JP, Naci H (2013) Is network meta-analysis as valid as standard pairwise meta-analysis? It al depends on the distribution of effect modifiers. BMCMed 11:159

435. Strøm T, Martinussen T, Toft P (2010) A protocol of no sedation for criti cally ill patients receiving mechanical ventilation: a randomised trial. Lancet 375:475-480

436. Lonardo NW, Mone MC, Nirula R et al (2014) Propofol is associated with favor-able Outcomes compared with benzodiazepines in ventilated intensive care unit patients. Am J Respir Crit Care Med 189:1383-1394

437. Fraser GL, Devlin JW, Worby CP et al (2013) Benzodiazepine versus non-benzodiazepinebased sedation for mechanically ventilated, critically ill adults: a systematic review and metaanalysis of randomized trials. Crit Care Med 41:S30-S38

438. DahLIA Investigators; Australian, New Zealand Intensive Care Society Clinical Trials Group, Reade MC, Eastwood GM, Bellomo R et al (2016) Effect of Dexmedetomidine Added to Standard Care on Ventilator-Free Time in Patients With Agitated Delirium: A Randomized Clinical Trial. JAMA 315:1460-1468

439. American College of Critical Care Medicine, Barr J, Fraser GL, Puntillo K et al (2013) Clinical practice guidelines for the management of pain, agitation, and delirium in adult patients in the intensive care unit. Crit Care Med 41:263-306

440. Leitlinie Analgesie, Sedierung und Delirmanagement in der Intensivmedizin, https://www.awmf org/leitlinien/detail/II/001-012.html aktueller Stand:08/2015

441. Van den Berghe G, Wouters P, Weekers Fetal (2001) Intensive insulin therapy in critically ill patients. NEngl J Med 345:1359-1367

442. Van den Berghe G, Wilmer A, Hermans Getal (2006) Intensive insulin therapy in the medical ICU. N Engl JMed 354:449-461

443. Arabi YM, Dabbagh OC, Tamim HM et al (2008) Intensive versus conven tional insulin therapy: a randomized controlled trial in medical and surgical critically ill patients. Crit Care Med 36:3190-3197

444. BrunkhorstFM, EngelC, Bloos Fetal (2008) German Competence Network Sepsis (SepNet): Intensive insulin therapy and pentastarch resuscitation in severe sepsis. N Engl J Med 358:125-139

445. De La Gdel RC, Donado JH, Restrepo AH et al (2008) Strict gly caemic control in patients hospitalised in a mixed medical and surgical intensive care unit: a randomised clinical trial. Crit Care 12(5):R120

446. Finfer S, Blair D, Bellomo R et al (2009) Intensive versus conven tional glucose control in critically ill patients. NEngl J Med 360(13):1283-1297

447. Annane D, Cariou A, Maxime V et al (2010) Corticosteroid treatment and intensive insulin therapy for septic shock in adults: a randomized controlled trial. JAMA 303(4):341-348

448. CGAO-REA Study Group, Kalfon P, Giraudeau B Ichai $C$ et al (2014) Tight computerized versus conventional glucose control in the ICU: a ran domized controlled trial. Intensive Care Med 40:171-181

449. Preiser JC, Devos P, Ruiz-Santana S et al (2009) A prospective ran domised multi-centre controlled trial on tight glucose control by intensive insulin therapy in adult intensive care units: the Glucontro study. Intensive Care Med 35:1738-1748

450. Zhang RH, Li T et al (2008) Evaluation of optimal goal of glucose con trol in critically ill patients. Chinese JClin Nutr 16:204-208

451. Friedrich JO, Chant C, Adhikari NK (2010) Does intensive insulin therapy really reduce mortality in critically ill surgical patients? A reanalysis of metaanalytic data. Crit Care 14:324

452. Griesdale DE, de Souza RJ, van Dam RM et al (2009) Intensive insulin therapy and mortality among critically ill patients: a meta-analysis including NICE-SUGAR study data. CMAJ 180:821-827

453. Kansagara D, Fu R, Freeman $M$ et al (2011) Intensive insulin therapy in hospitalized patients: a systematic review. Ann Intern Med 154:268-282

454. Marik PE, Preiser JC (2010) Toward understanding tight glycemic control in the ICU: a systematic review and metaanalysis. Chest 137:544-551

455. Wiener RS, Wiener DC, Larson RJ (2008) Benefits and risks of tight glu cose control in critically ill adults: a meta-analysis. JAMA 300:933-944

456. Ling Y, Li X, Gao X (2012) Intensive versus conventional glucose control in critically ill patients: a meta-analysis of randomized controlled trials. Eur J Intern Med 23:564-574

457. Song F, Zhong LJ, Han L et al (2014) Intensive insulin therapy for septic patients: a meta-analysis of randomized controlled trials. Biomed Res Int 2014:698265

458. American Diabetes Association (2014) Standards of medical care in diabe tes-2014. Diabetes Care 37(Suppl 1):S14-S80

459. Marvin MR, Inzucchi SE, Besterman BJ (2013) Computerization of the Yale insulin infusion protocol and potential insights into causes of hypoglycemia with intravenous insulin. Diabetes Technol Ther 15:246-252

460. Clinical Guidelines Committee of the American College of Physicians, Qaseem A, Chou R, Humphrey LL et al (2014) Inpatient gly cemic control: best practice advice from the Clinical Guidelines Committee of the American College of Physicians. Am J Med Qual 29:95-98

461. Siegelaar SE, Hermanides J, Oudemans-van Straaten HM et al (2010) Mean glucose during ICU admission is related to mortality by a Ushaped curve in surgical and medical patients: a retrospective cohort study. Crit Care 14:R224

462. Badawi O, Waite MD, Fuhrman SA et al (2012) Association between intensive care unit-acquired dysglycemia and in-hospital mortality. Crit Care Med 40:3180-3188

463. Finfer S, Liu B, Chittock DR et al (2012) Hypoglycemia and risk of death in critically ill patients. N Engl JMed 367(12):1108-1118 
464. CGAO-REA Study Group, Kalfon P, Le Manach Y, Ichai C et al (2015) Severe and multiple hypoglycemic episodes are associated with increased risk of death in ICU patients. Crit Care 19:153

465. Krinsley JS (2008) Glycemic variability: a strong independent predic tor of mortality in critically ill patients. Crit Care Med 36:3008-3013

466. Todi S, Bhattacharya M (2014) Glycemic variability and outcome in critically ill. Indian J Crit Care Med 18:285-290

467. Kauffmann RM, Hayes RM, Jenkins JM et al (2011) Provision of balanced nutrition protects against hypoglycemia in the critically ill surgical patient. JPEN J Parenter Enteral Nutr 35:686-694

468. Egi M, Bellomo R, Stachowski E et al (2008) Blood glucose concentration and outcome of critical illness: the impact of diabetes. Crit Care Med 36:2249-2255

469. Krinsley JS (2009) Glycemic variability and mortality in critically ill patients: the impact of diabetes. JDiabetes Sci Technol 3:1292-1301

470. Krinsley JS, Preiser JC (2015) Time in blood glucose range 70 to $140 \mathrm{mg} / \mathrm{dl}>80 \%$ is strongly associated with increased survival in non-diabetic critically ill adults. Crit Care 19:179

471. Egi M, Bellomo R, Stachowski E et al (2011) The interaction of chronic and acute glycemia with mortality in critically ill patients with diabetes. Crit Care Med 39:105-111

472. Sandler V, Misiasz MR, Jones J et al (2014) Reducing the risk of hypogly cemia associated with intravenous insulin: experience with a com puterized insulin infusion program in 4 adult intensive care units. J Diabetes Sci Technol 8:923-929

473. Pereira AJ, Corrêa TD, de Almeida FP et al (2015) Inaccuracy of venous point-of-care glucose measurements in critically ill patients: a crosssectional study. PLoS ONE 10:e129568

474. Hoedemaekers CW, Klein Gunnewiek JM, Prinsen MA et al (2008) Accuracy of bedside glucose measurement from three glucometers in critically ill patients. Crit Care Med 36:3062-3066

475. Inoue S, Egi M, Kotani J et al (2013) Accuracy of blood-glucose measure ments using glucose meters and arterial blood gas analyzers in criti cally ill adult patients: systematic review. Crit Care 17:R48

476. Kanji S, Buffie J, Hutton B et al (2005) Reliability of point-of-care testing for glucose measurement in critically ill adults. Crit Care Med 33:2778-2785

477. Khan AI, Vasquez Y, Gray Jetal (2006) The variability of results between point-of-care testing glucose meters and the central laboratory ana lyzer. Arch Pathol Lab Med 130:1527-1532

478. Rice MJ, Coursin DB (2016) Glucose meters: here today, gone tomorrow? Crit Care Med 44:e97-e100

479. Wilson M, Weinreb J, Hoo GW (2007) Intensive insulin therapy in critical care: a review of 12 protocols. Diabetes Care 30:1005-1011

480. Dortch MJ, Mowery NT, Ozdas A et al (2008) A computerized insulin infu sion titration protocol improves glucose control with less hypogly cemia compared to a manual titration protocol in a trauma intensive care unit. JPEN J Parenter Enteral Nutr 32:18-27

481. Newton CA, Smiley D, Bode BW et al (2010) A comparison study of con tinuous insulin infusion protocols in the medical intensive care unit: computer-guided vs. standard column-based algorithms. JHosp Med 5:432-437

482. Bartlett RH, Mault JR, Dechert RE et al (1986) Continuous arteriovenous hemofiltration: improved survival in surgical acute renal failure? Surgery 100:400-408

483. Bellomo R, Farmer M, Parkin G et al (1995) Severe acute renal failure: a comparison of acute continuous hemodiafiltration and conventional dialytic therapy. Nephron 71:59-64

484. Bellomo R, Mansfield D, Rumble S et al (1992) Acute renal failure in critical illness. Conventional dialysis versus acute continuous hemodiafiltration. ASAIO J 38:M654-M657

485. Kierdorf H (1991) Continuous versus intermittent treatment: clinical results in acute renal failure. In: Sieberth HG, Mann H, Stummvoll HK (Hrsg) Continuous Hemofiltration. Karger, Basel, S1-12

486. Mauritz W, Sporn P, Schindler I et al (1986) Acute renal failure in abdominal infection. Comparison of hemodialysis and continuous arteriovenous hemofiltration. Anasth Intensivther Notfallmed 21:212-217

487. Guérin C, Girard R, Selli JM et al (2002) Intermitten versus continuous renal replacement therapy for acute renal failure in intensive care units: results from a multicenter prospective epidemiological survey. Intensive Care Med 28:1411-1418

488. van Bommel E, Bouvy ND, So KL et al (1995) Acute dialytic support for the critically ill: intermittent hemodialysis versus continuous arterio venous hemodiafiltration. Am JNephrol 15:192-200

489. Kellum JA, Angus DC, Johnson JP et al (2002) Continuous versus intermit tent renal replacement therapy: a meta-analysis. Intensive Care Med 28:29-37

490. Tonelli M, Manns B, Feller-Kopman D (2002) Acute renal failure in the inten sive care unit: a systematic review of the impact of dialytic modality on mortality and renal recovery. Am J Kidney Dis 40:875-885

491. Augustine JJ, Sandy D, Seifert TH et al (2004) A randomized controlled trial comparing intermittent with continuous dialysis in patients with ARF. Am J Kidney Dis 44:1000-1007

492. Gasparovi V, Filipovi-Grci I, Merkler M, : et al (2003) Continuous renal replacement therapy (CRRT) or intermittent hemodialysis (IHD) — what is the procedure of choice in critically ill patients? Ren Fail 25:855-862

493. Collaborative Group for Treatment of ARF in the ICU, Mehta RL, McDonald B, Gabbai FB et al (2001) A randomized clinical trial of continu ous versus intermittent dialysis for acute renal failure. Kidney Int 60:1154-1163

494. Uehlinger DE, Jakob SM, Ferrari $P$ et al (2005) Comparison of continuous and intermittent rena replacement therapy for acute renal failure. Nephrol Dial Transplant 20:1630-1637

495. Hemodiafe Study Group, Vinsonneau C, Camus C Combes A et al (2006) Continuous venovenous haemodiafiltration versus intermittent hae modialysis for acute renal failure in patients with multiple-organ dysfunction syndrome: a multicentre randomised trial. Lancet 368:379-385

496. John S, Griesbach D, Baumgärtel M et al (2001) Effects of continuous haemofiltration vs intermittent haemodialysis on systemic haemody namics and splanchnic regional perfusion in septic shock patients: a prospective, randomized clinical trial. Nephrol Dial Transplant 16:320-327

497. Misset B, Timsit JF, Chevret S et al (1996) A randomized cross-over comparison of the hemodynamic response to intermittent hemodialysis and continuous hemofiltration in ICU patients with acute renal failure. Intensive Care Med 22:742-746
498. Bellomo R, Cass A, Cole L et al (2009) Intensity of continuous renal replacement therapy in critically ill patients. NEngl J Med 361(17):1627-1638

499. Palevsky PM, Zhang JH, O'Connor TZ et al (2008) Intensity of renal sup port in critically ill patients with acute kidney injury. NEngl J Med 359(1):7-20

500. Bouman CS, Oudemans-Van Straaten HM, Tijssen JG et al (2002) Effects of early highvolume continuous venovenous hemofiltration on sur $\neg$ vival and recovery of renal function in intensive care patients with acute renal failure: a prospective, randomized trial. Crit Care Med 30:2205-2211

501. Zarbock A, Kellum JA, Schmidt C et al (2016) Effect of early vs delayed initiation of renal replacement therapy on mortality in critically ill patients with acute kidney injury: the ELAIN randomized clinical trial.JAMA 315:2190-2199

502. AKIKIStudy Group, Gaudry S, Hajage D, Schortgen F et al (2016) Initiation strategies for renalreplacement therapy in the intensive care unit NEngl J Med 375:122-133

503. Cooper DJ, Walley KR, Wiggs BR et al (1990) Bicarbonate does not improve hemodynamics in critically ill patients who have lactic acidosis. A prospective, controlled clinical study. Ann Intern Med 112:492-498

504. Mathieu D, Neviere R, Billard V et al (1991) Effects of bicarbonate therapy on hemodynamics and tissue oxygenation in patients with lactic aci dosis: a prospective, controlled clinical study. Crit Care Med 19:1352-1356

505. Cook D, Crowther M, Meade M et al (2005) Deep venous thrombosis in medical-surgical critically ill patients: prevalence, incidence, and risk factors. Crit Care Med 33:1565-1571

506. Kahn SR, Lim W, Dunn AS et al (2012) Prevention of VTE in nonsurgical patients: antithrombotic therapy and prevention of thrombosis, 9th ed: American College of Chest Physicians evidencebased clinical practice guidelines. Chest $141(2$ Suppl):e195S-e226S

507. Alhazzani W, Lim W, Jaeschke RZ et al (2013) Heparin thromboprophy laxis in medical-surgical critically ill patients: a systematic review and meta-analysis of randomized trials. Crit Care Med 41:2088-2098

508. Duranteau J et al (2018) Eur J Anaesthesiol 35:142-146. https://doi.org/10.1097/EJA. 0000000000000707

509. Xigris and Prophylactic HepaRin Evaluation in Severe Sepsis (XPRESS) Study Group, Levi M, Levy M, Williams MD et al (2007) Prophylactic heparin in patients with severe sepsis treated with drotrecogin alfa (activated). Am J Respir Crit Care Med 176:483-490

510. Beitland S, Sandven I, Kjærvik LK et al (2015) Thromboprophylaxis with low molecular weight heparin versus unfractionated heparin in intensive care patients: a systematic review with metaanalysis and trial sequential analysis. Intensive Care Med 41:1209-1219

511. Junqueira DR, Perini E, Penholati RR, Carvalho MG (2012) Unfractionated heparin versus low molecular weight heparin for avoiding heparin induced thrombocytopenia in postoperative patients. Cochrane Database Syst Rev. https://doi.org/10 1002/14651858.CD007557.pub2

512. Phung OJ, Kahn SR, Cook DJ et al (2011) Dosing frequency of unfrac tionated heparin thromboprophylaxis: a meta-analysis. Chest 140:374-381

513. Mahan CE, Pini M, Spyropoulos AC (2010) Venous thromboembolism prophylaxis with 
unfractionated heparin in the hospitalized medi cal patient: the case for thrice daily over twice daily dosing. Intern Emerg Med 5:299-306

514. Hull RD et al (1986) Continuous intravenous heparin compared with intermittent subcutaneous heparin in the initial treatment of proximal-vein thrombosis. NEngl J Med 315:1109-1114

515. Mayr AJ et al (2002) Antifactor Xa activity in intensive care patients receiving thromboembolic prophylaxis with standard doses of enoxaparin Thromb Res 105(3):201-204

516. Lim SY et al (2013) Antifactor Xa levels in critically ill Korean patients receiving enoxaparin for thromboprophylaxis: a prospective observational study. J Korean Med Sci 28(3):466-471. https://doi. org/10.3346/jkms.2013.28.3.466

517. Jochberger S et al (2005) Antifactor Xa activity in critically ill patients receiving antithrombotic prophylaxis with standard dosages of certoparin: a prospective, clinical study. Crit Care 9(5):R541-R548. https://doi.org/10.1186/cc3792

518. Robinson $S$ et al (2013) A comparative study of varying doses of enoxaparin for thromboprophylaxis in critically ill patients: double-blinded, randomised controlled trial. Crit Care 17(2):R75. https://doi.org/10.1186/cc12684

519. Lim W et al (2006) Meta-analysis: low-molecularweight heparin and bleeding in patients with severe renal insufficiency. Ann Intern Med 144(9):673-684

520. Rabbat CG et al (2005) Dalteparin thromboprophylaxis for critically ill medical-surgical patients with renal insufficiency. J Crit Care 20:357-363

521. Canadian Critical Care Trials Group, Douketis J, Cook D, Meade M et al (2008) Prophylaxis against deep vein thrombosis in critically ill patients with severe renal insufficiency with the low-molecularweight heparin dalteparin: an assessment of safety and pharmacodynamics: the DIRECT study. Arch Intern Med 168:1805-1812

522. Cook D, Meade M, Guyatt G et al (2011) Dalteparin versus unfrac tionated heparin in critically ill patients. NEngl J Med 364(14):1305-1314

523. Canadian Critical Care Trials Group, Australia and New Zealand Intensive Care Society Clinical Trials Group, Fowler RA, Mittmann N, Geerts W et al (2014) Cost-effectiveness of dalteparin vs unfraction ated heparin for the prevention of venous thromboembolism in criti cally ill patients. JAMA 312:2135-2145

524. Leitlinie „Prophylaxe der venösen Thromboembolie (VTE)" (AWMF-Leitlinien Register-Nr. 003/001)

525. Kakkos SK, Caprini JA, Geroulakos G, Nicolaides AN, Stansby GP, Reddy DJ (2008) Combined intermittent pneumatic leg compression and pharmacological prophylaxis for prevention of venous throm boembolism in high-risk patients. Cochrane Database Syst Rev. https://doi.org/10. 1002/14651858.CD005258.pub2

526. Kakkos SK et al (2016) Cochrane Database Syst Rev. https://doi.org/10.1002/14651858.CD005258. pub2

527. Falck-Ytter Y, Francis CW, Johanson NA et al (2012) Prevention of VTE in orthopedic surgery patients: Antithrombotic Therapy and Prevention of Thrombosis, 9th ed: American College of Chest Physicians evidence-based clinical practice guidelines. Chest 141(2 Suppl):e278S-e325S

528. Gould MK, Garcia DA, Wren SM et al (2012) Prevention of VTE in nonorthopedic surgical patients: Antithrombotic Therapy and Prevention of Thrombosis, 9th ed: American College of Chest Physicians Evidence-Based Clinical Practice Guidelines. Chest 141(2 Suppl):e227S-e277S
529. Arabi YMetal (2018) Trials 19:182

530. Arabi YM, Alsolamy S, Al-Dawood A et al (2016) Thromboprophylaxisusing combinedintermittent pneumatic compression and pharmaco logic prophylaxis versus pharmacologic prophylaxis alone in critically ill patients: study protocol for a randomized controlled trial. Trials 17:390

531. Pavon JM, Adam SS, Razouki ZA et al (2016) Effectiveness of intermittent pneumatic compression devices for venous thromboembolism prophylaxis in high-risk surgical patients: a systematic review. J Arthroplasty 31:524-532

532. Sachdeva A, Dalton M, Amaragiri SV, Lees T (2014) Graduated compression stockings for prevention of deep vein thrombosis. Cochrane Database Syst Rev. https://doi.org/10.1002/14651858. CD001484.pub3

533. Arabi YM, Khedr M, Dara SI et al (2013) Use of intermittent pneumatic compression and not graduated compression stockings is associated with lower incident VTE in critically ill patients: a multiple propensity scores adjusted analysis. Chest 144:152-159

534. Canadian Critical Care Trials Group, Cook DJ, Griffith LE, Walter SD et al (2001) The attributable mortality and length of intensive care unit stay of clinically important gastrointestinal bleeding in critically ill patients. Crit Care 5:368-375

535. Bardou M, Quenot JP, Barkun A (2015) Stressrelated mucosal disease in the critically ill patient. Nat Rev Gastroenterol Hepatol 12:98-107

536. Cook DJ, Fuller HD, Guyatt GH et al (1994) Risk factors for gastrointes tinal bleeding in critically ill patients. Canadian Critical Care Trials Group. NEngl JMed 330:377-381

537. SUP-ICU co-authors, Krag M, Perner A, Wetterslev et al (2015) Prevalence and Outcome of gastrointestinal bleeding and use of acid suppressants in acutely ill adult intensive care patients. Intensive Care Med 41:833-845

538. Andersson B, Nilsson J, Brandt J et al (2005) Gastrointestinal complica tions after cardiac surgery. Br J Surg 92:326-333

539. Bruno JJ, Canada TW, Wakefield CD et al (2009) Stress-related mucosal bleeding in critically ill oncology patients. JOncol Pharm Pract 15:9-16

540. D'Ancona G, Baillot R, Poirier B et al (2003) Determinants of gastrointestinal complications in cardiac surgery. Tex Heart Inst J 30:280-285

541. Faisy C, Guerot E, Diehl JL et al (2003) Clinically significant gastrointes tinal bleeding in critically ill patients with and without stress-ulcer prophylaxis. Intensive Care Med 29:1306-1313

542. Krag M, Perner A, Wetterslev J et al (2014) Stress ulcer prophylaxis versus placebo or no prophylaxis in critically ill patients. A systematic review of randomised clinical trials with meta-analysis and trial sequential analysis. Intensive Care Med 40:11-22

543. Sasabuchi Y, Matsui H, Lefor AK et al (2016) Risks and benefits of stress ulcer prophylaxis for patients with severe sepsis. Crit Care Med 44:e464-e469

544. Eastwood GM, Litton E, Bellomo R et al (2014) Opinions and practice of stress ulcer prophylaxis in Australian and New Zealand intensive care units. Crit Care Resusc 16:170-174

545. Preslaski CR, Mueller SW, Kiser TH et al (2014) A survey of prescriber perceptions about the prevention of stress-related mucosal bleeding in the intensive care unit. J Clin Pharm Ther 39:658-662

546. Shears M, Alhazzani W, Marshall JC et al (2016) Stress ulcer prophy laxis in critical illness: a Canadian survey. Can J Anaesth 63:718-724
547. Alshamsi F, Belley-Cote E, Cook D et al (2016) Efficacy and safety of proton pump inhibitors for stress ulcer prophylaxis in critically ill patients: a systematic review and meta-analysis of randomized trials. Crit Care 20:120

548. Alhazzani W, Alenezi F, Jaeschke RZ et al (2013) Proton pump inhibitors versus histamine 2 receptor antagonists for stress ulcer prophylaxis in critically ill patients: a systematic review and metaanalysis. Crit Care Med 41:693-705

549. Barkun AN, Bardou M, Pham CQ, Martel M (2012) Proton pump inhibi tors vs. histamine 2 receptor antagonists for stress-related mucosal bleeding prophylaxis in criticallyill patients: a meta-analysis. Am J Gastroenterol 107(4):507-520 (quiz 21)

550. AlhazzaniW etal (2018) Efficacy and safety of stress ulcer prophylaxis in critically ill patients: a network meta-analysis of randomized trials. Intensive Care Med 44(1):1-11

551. Barkun AN, Adam V, Martel M et al (2013) Cost-effectiveness analysis: stress ulcer bleeding prophylaxis with proton pump inhibitors, $\mathrm{H} 2$ receptor antagonists. Value Health 16:14-22

552. MacLaren R, Campbell J (2014) Cost-effectiveness of histamine receptor- 2 antagonist versus proton pump inhibitor for stress ulcer prophylaxis in critically ill patients. Crit Care Med 42:809-815

553. Alhazzani W et al (2017) Crit Care Med 45(7):1121-1129

554. Selvanderan et al (2016) Crit Care Med 44(10):1842-1850

555. Krag et al (2018) NEng J Med 379(23):2199-2208

556. Hartl et al (2013) Aktuel Ernahrmed 38:e90-e100

557. Elke G, Hartl WH, Kreymann KG, Adolph , Felbinger TW, Graf T, de Heer G, Heller AR, Kampa U, Mayer K, Muhl E, Niemann B, Rümelin A, Steiner S, Stoppe C, Weimann A, Bischoff SC (2018) DGEM Leitlinie Klinische Ernährung in der Intensivmedizin. Aktuel Ernahrungsmed 43:341-408

558. Reintam Blaser A, Berger MM (2017) Early or late feeding after ICU admission? Nutrients 23(9):12-E1278. https://doi.org/10.3390/ nu9121278

559. Singer $P$, Reintam Blaser A, Berger MM, AlhazzaniW Calder $\mathrm{P}$, Casaer M, Hiesmayr M, Mayer $\mathrm{K}$, Montejo JC, Pichard C, Preiser JC, van Zanten ARH, Oczkowski S, Szczeklik W, Bischoff SC (2018) ESPEN Guideline Clinical nutrition in the intensive care unit. Clin Nutr. https://doi.org/10.1016/j.clnu. 2018.08.037

560. Weimann A, Felbinger T (2016) Gastrointestinal dysmotility in the critically ill-a role for nutrition. Curr Opin Clin Nutr Metab Care 19:353-359

561. Borzotta AP, Pennings J, Papasadero B et al (1994) Enteral versus parenteral nutrition after severe closed head injury. JTrauma 37:459-468

562. Adams S, Dellinger EP, Wertz MJ et al (1986) Enteral versus parenteral nutritional support following laparotomy for trauma: a randomized prospective trial. JTrauma 26:882-891

563. Dunham CM, Frankenfield D, Belzberg $\mathrm{H}$ et al (1994) Gut failure-predictor of or contributor to mortality in mechanically ventilated blunt trauma patients? JTrauma 37:30-34

564. CALORIES Trial Investigators, Harvey SE, Parrott F, Harrison DA et al (2014) Trial of the route of early nutritional support in critically ill adults. N Engl J Med 371:1673-1684

565. Justo Meirelles CM, de Aguilar-Nascimento JE (2011) Enteral or parenteral nutrition in traumatic brain injury: a prospective randomised trial. Nutr Hosp 26:1120-1124 
566. Kalfarentzos F, Kehagias J, Mead N et al (1997) Enteral nutrition is superior to parenteral nutrition in severe acute pancreatitis:results of a randomized prospective trial. Br J Surg 84:1665-1669

567. Moore FA, Moore EE, Jones TN, McCroskey BL, Peterson VM (1989) TEN versus TPN following major abdominal trauma—reduced septic morbidity. JTrauma 29(7):916-922 (discussion 22-23)

568. Peterson VM, Moore EE, Jones TN et al (1988) Total enteral nutrition versus total parenteral nutrition after major torso injury: attenuation of hepatic protein reprioritization. Surgery 104:199-207

569. Sun JK, Mu XW, Li WQ et al (2013) Effects of early enteral nutrition on immune function of severe acute pancreatitis patients. World J Gastroenterol 19:917-922

570. Wang G, Wen J, Xu L et al (2013) Effect of enteral nutrition and ecoim-munonutrition on bacterial translocation and cytokine production in patients with severe acute pancreatitis. J Surg Res 183:592-597

571. Lewis SR, Schofield-Robinson OJ, Alderspn P, Smith AF (2018) Enteral versus parenteral nutrition and enteral versus a combination of enteral and parenteral nutrition for adults in the intensive care unit. Cochrane Database Syst Rev. https://doi.org/ 10.1002/14651858.CD012276.pub2

572. Harvey SE, Parrott F, Harrison DA et al (2016) A multicentre, randomised controlled trial comparing the clinical effectiveness and cost-effectiveness of early nutritional support via the parenteral versus the enteral route in critically ill patients (CALORIES). Health Technol Assess 20:1-144

573. Elke G, van Zanten AR, Lermieux M, McCall M, Jeejeebhoy KN, Kott M, Jiang X, Day AG, Heyland DK (2016) Enteral versus parenteral nutrition in critically ill patients: an updated systematic review and meta-analysis of randomized controlled trials. Crit Care 20(1):117. https://doi.org/10.1186/ s13054-016-1298-11

574. Tian F, Wang X, Gao X, Wan X, Wu C, Zhang L, Li N, Li J (2015) Effect of initial caloric intake via enteral nutrition in critical illness: a meta-analysis of randomised controlled trials. Crit Care 19:180

575. Zhang G, Zhang K, Cui W, Hong Y, Zhang Z (2018) The effect of enteral versus parenteral nutrition for critically ill patients: a systematic review and metaanalysis. JClin Anesth 51:62-92

576. Reignier J, Boisramé-Helms J, Brisard L, Lascarrou JB, Ait Hssain A, Anguel N, Argaud L, Asehnoune K, AsfarP, BellecF, Botoc V, Bretagnol A, Bui HN, Canet E, Da Silva D, Darmon M, Das V, Devaquet J, Djibre M, Ganster F, Garrouste-Orgeas M, Gaudry S, Gontier O, Guérin C, Guidet B, Guitton C, Herbrecht JE, Lacherade JC, Letocart P, Martino F, Maxime V, Mercier E, Mira JP, Nseir S, Piton G, Quenot JP, Richecoeur J, Rigaud JP, Robert R, Rolin N, Schwebel C, Sirodot $M$, Tinturier F, Thévenin D, Giraudeau B, Le Gouge A (2018) Enteral versus parenteral early nutrition in ventilated adults with shock: a randomised, controlled, multicentre, open-label, parallel-group study (NUTRIREA-2). NUTRIREA-2 Trial Investigators; Clinical Research in Intensive Care and Sepsis (CRICS) group. Lancet 391(10116):133-143. https://doi.org/10.1016/ S0140-6736(17)32146-3

577. Adams S (1986) JTrauma. https://doi.org/10.1097/ 00005373-198610000-00004

578. Kudsk KA (2002) Current aspects of mucosal immunology and its influence by nutrition. Am J Surg 183:390-398

579. Eyer SD, Micon LT, Konstantinides FN et al (1993) Early enteral feeding does not attenuate metabolic response after blunt trauma. J Trauma 34:639-643 (discussion 643)

580. DvorakMF, Noonan VK, Bölanger Letal (2004) Early versus late enteral feeding in patients with acute cervical spinal cord injury: a pilot study. Spine 29:E175-E180

581. Grahm TW, Zadrozny DB, Harrington T (1989) The benefits of early jejunal hyperalimentation in the head-injured patient. Neurosurgery 25:729-735

582. Hasse JM, Blue LS, Liepa GU et al (1995) Early enteral nutrition support in patients undergoing liver transplantation. JPEN J Parenter Enteral Nutr 19:437-443

583. Minard G, Kudsk KA, Melton S et al (2000) Early versus delayed feeding with an immuneenhancing diet in patients with severe head injuries. JPEN J Parenter Enteral Nutr 24:145-149

584. Moore EE, Jones TN (1986) Benefits of immediate jejunostomy feeding after major abdominal trauma-a prospective, randomized study. J Trauma 26:874-881

585. Singh G, Ram RP, Khanna SK (1998) Earlypostoperative enteral feeding in patients with nontraumatic intestinal perforation and peritonitis. J Am Coll Surg 187:142-146

586. Nguyen NQ, Fraser RJ, Bryant LK et al (2008) The impact of delaying enteral feeding on gastric emptying, plasma cholecystokinin, and peptide YY concentrations in critically ill patients. Crit Care Med 36:1469-1474

587. Peng YZ, Yuan ZQ, Xiao GX (2001) Effects of early enteral feeding on the prevention of enterogenic infection in severely burned patients. Burns 27:145-149

588. Chuntrasakul C, Chinswangwatanakul V, Chockvivatanavanit $S$, Siltharm S, Pongprasobchai $T$, BunnakA (1996) Early nutritional support in severe traumatic patients. J Med Assoc Thai 79(1):21-26

589. Chourdakis M, Kraus MM, TzellosTetal (2012) Effect of early compared with delayed enteral nutrition on endocrine function in patients with traumatic brain injury: an open-labeled randomized trial. JPEN J Parenter Enteral Nutr 36:108-116

590. Doig GS, Heighes PT, Simpson F et al (2011) Early enteral nutrition reduces mortality in trauma patients requiring intensive care: a metaanalysis of randomised controlled trials. Injury 42:50-56

591. Doig GS, Heighes PT, Simpson F et al (2009) Early enteral nutrition, provided within $24 \mathrm{~h}$ of injury or intensive care unit admission, significantly reduces mortality in critically ill patients: a meta-analysis of randomised controlled trials. Intensive Care Med 35:2018-2027

592. Malhotra A, Mathur AK, Gupta S (2004) Early enteral nutrition after surgical treatment of gut perforations: a prospective randomised study. JPostgrad Med 50:102-106

593. Pupelis G, Austrums E, Jansone A et al (2000) Randomised trial of safety and efficacy of postoperative enteral feeding in patients with severe pancreatitis: preliminary report. Eur J Surg 166:383-387

594. Arabi YM, Aldawood AS, Haddad SH et al (2015) PermiT trial group: permissive underfeeding or standard enteral feeding in critically ill adults. NEngl J Med 372:2398-2408

595. Marik PE, Hooper MH (2016) Normocaloric versus hypocaloric feeding on the Outcomes of ICU patients: a systematic review and meta-analysis. Intensive Care Med 42:316-323

596. Arabi YM, Tamim HM, Dhar GS et al (2011) Permissive underfeeding and intensive insulin therapy in critically ill patients: a randomized controlled trial. Am J Clin Nutr 93:569-577
597. Charles EJ, Petroze RT, Metzger R et al (2014) Hypocaloric compared with eucaloric nutritional support and its effect on infection rates in a surgical intensive care unit: a randomized controlled trial. Am J Clin Nutr 100:1337-1343

598. Ibrahim EH, Mehringer L, Prentice D et al (2002) Early versus late enteral feeding of mechanically ventilated patients: results of a clinical trial. JPEN Parenter Enteral Nutr 26:174-181

599. National Heart, Lung, and Blood Institute Acute Respiratory Distress Syndrome (ARDS) Clinical Trials Network, Rice TW, Wheeler AP et al (2012) Initial trophic vs full enteral feeding in patients with acute lung injury: the EDEN randomized trial. JAMA 307(8):795-803

600. Petros S, Horbach M, Seidel F et al (2016) Hypocaloric vs normocaloric nutrition in critically ill patients: a prospective randomized pilot trial. JPEN J Parenter Enteral Nutr 40:242-249

601. RiceTW, MoganS, Hays MAetal(2011)Randomized trial of initial trophic versus full-energy enteral nutrition in mechanically ventilated patients with acute respiratory failure. Crit Care Med 39:967-974

602. Needham DM, Dinglas VD, Bienvenu OJ et al (2013) One year Outcomes in patients with acute lung injury randomised to initial trophic or full enteral feeding: prospective followup of EDEN randomised trial. BMJ 346:f1532

603. Ridley EJ, Davies AR, Hodgson CL, Deane A, Bailey M, Cooper DJ (2017) Delivery of full predicted energy from nutrition and the effect on mortality in critically ill adults: a systematic review and meta-analysis of randomised controlled trials. Clin Nutr. https://doi.org/10.1016/j.clnu.2017.09. 026

604. Choi EY, Park DA, Park J (2015) Calorie intake of enteral nutrition and clinical Outcomes in acutely criticallyill patients: a meta-analysis of randomized controlled trials. JPEN J Parenter Enteral Nutr 39:291-300

605. Casaer MP, Mesotten D, Hermans G et al (2011) Early versus late parenteral nutrition in critically il adults. NEngl J Med 365:506-517

606. Early PN Investigators of the ANZICS Clinical Trials Group, Doig GS, Simpson F, Sweetman EA et al (2013) Early parenteral nutrition in critically ill patients with short-term relative contraindications to early enteral nutrition: a randomized controlled trial. JAMA 309:2130-2138

607. Young B, Ott L, Haack D et al (1987) Effect of total parenteral nutrition upon intracranial pressure in severe head injury. J Neurosurg 67:76-80

608. Rapp RP, Donaldson ES, Bivins BA (1983) Parentera nutrition in a patient with familial Type IV hypertriglyceridemia: a dilemma. Drug Intell Clin Pharm 17(6):458-460

609. Sadique Z, Grieve R, Harrison D et al (2015) Costeffectiveness of early parenteral versus enteral nutrition in critically ill patients. Value Health 18:A532

610. The TARGET Investigators for the ANZICS Clinical Trials Group. (2018) Energy-dense versus routine enteral nutrition in the critically ill. N Engl J Med 379:823-834

611. Allingstrup MJ, Kondrup J, Wiis J, Claudius C, Pedersen UG, Hein-Rasmussen R, Bjerregaard MR, Steensen $M$, Jensen $T H$, Lange $T$, Madsen $M B$, Møller MH, Perner A (2017) Early goal-directed nutrition versus standard of care in adult intensive care patients: the single-centre, randomised, Outcome assessor-blinded EAT-ICU trial. Intensive Care Med 43(11):1637-1647. https://doi.org/10. 1007/s00134-017-4880-3 
612. Stuani Franzosi O, Delfino von Frankenberg $A$, Loss SH, Solva Leite Nunes D, Rios Vieiira SR (2017) Underfeeding versus full enteral feeding in critically ill patients witrh acute respirartoryx failure: a systematic review with meta-analyis of randomized controlled trials. Nutr Hosp 34:19-29

613. Yeh DD, Fuentes E, Quraishi SA, Cropano C, Kaafarani H, Lee J, King DR, DeMoya M, FagenholzP, Butler K, Chang Y, Velmahos G (2016) Adequate nutrition may get you home: effect of caloric/ protein deficits on the discharge destination of criticallyill surgical patients.JPENJ Parenter Enteral Nutr 40:37-44

614. Nicolo M, Heyland DK, Crittiams J, Sammarco T, Compher C (2016) Clinical Outcomes related toprotein delivery in a critically ill population: a multicenter,multinational observational study. JPEN JParenter Enteral Nutr 40(1):45-51

615. Zusman O, Theilla M, Cohen J, Kagan I, Bendavid I, Singer $P(2016)$ Resting energy expenditure, calorie and protein consumption in critically ill patients: a retrospective cohort study. Crit Care 20(1):367

616. Compher C, Chittams J, Sammero T, Nicolo M Heyland DK (2017) Greater protein and energy intake may be associated with improved mortality in higher risk critically ill patients: a multicenter multinational observational study. Crit Care Med 41:104-112

617. HartIWH, Bender A, ScheiplF, KuppingerD, Day AG, Küchenhoff H (2018) Caloriei intake and shortterm survival of critically ill patients. Clin Nutr. https://doi.org/10.1016/j.clnu.2018.04.005

618. A.S.P.E.N. Board of Directors, American College of Critical Care Medicine, Society of Critical Care Medicine Guidelines for the Provision, Assessment of Nutrition Support Therapy in the Adult Critically III Patient, Society of Critical Care Medicine (SCCM), American Society for Parenteral and Enteral Nutrition (A.S.P.E.N.), McClave SA, McClave SA, Taylor BE, Martindale RG, Warren MM et al (2016) Important and most recent guideline regarding clinical nutrition in the ICU. JPEN J Parenter Enteral Nutr 40:159-211

619. McClave SA, DeMeo MT, DeLegge MH et al (2002) North American Summit on Aspiration in the Critically III Patient: consensus statement. JPEN J Parenter Enteral Nutr 26:S80-S85

620. McClave SA, Lukan JK, Stefater JA et al (2005) Poor validity of residual volumes as a marker for risk of aspiration in critically ill patients. Crit Care Med 33:324-330

621. Metheny NA, Schallom L, Oliver DA, Clouse RE (2008) Gastric residual volume and aspiration in critically ill patients receiving gastric feedings. Am JCrit Care 17(6):512-519 (quiz 20)

622. Montejo JC, Miñambres E, Bordejö L et al (2010) Gastric residual volume during enteral nutrition in ICU patients: the REGANE study. Intensive Care Med 36:1386-1393

623. Poulard F, Dimet J, Martin-Lefevre L et al (2010) Impact of not measuring residual gastric volume in mechanically ventilated patients receiving early enteral feeding: a prospective before-after study. JPEN J Parenter Enteral Nutr 34:125-130

624. Clinical Research in Intensive Care and Sepsis (CRICS) Group, Reignier J, Mercier E, Le Gouge A et al (2013) Effect of not monitoring residual gastric volume on risk of ventilator-associated pneumonia in adults receiving mechanical ventilation and early enteral feeding: a randomized controlled trial. JAMA 309:249-256

625. Hartl et al (2013) Aktuel Ernährmed 38:e90-e100 (https://www.dgem.de/sites/default/files/PDFs/ Leitlinien/S3-Leitlinien/073-022I_S3_\%C3
\%9Cberwachung bei_k\%C3\%BCnstlicher_Ern \%C3\%A4hrung_2013-10.pdf)

626. van Noord C, Dieleman JP, van Herpen G et al (2010) Domperidone and ventricular arrhythmia or sudden cardiac death: a population-based casecontrol study in the Netherlands. Drug Saf 33:1003-1014

627. Ray WA, Murray KT, Meredith S et al (2004) Oral erythromycin and the risk of sudden death from cardiac causes. N Engl J Med 351:1089-1096

628. Lewis K, Alqahtani Z, Mcintyre L et al (2016) The efficacy and safety of prokinetic agents in critically ill patients receiving enteral nutrition: a systematic review and meta-analysis of randomized trials. Crit Care 20:259

629. BDDixon DJCoopersmith FCM et al (2008) Pharmacoepidemiology of QT-interval prolonging drug administration in critically ill patients. Pharmacoepidemiol Drug Saf 17:971-981

630. Fruhwald S (2010) Curr Opin Crit Care. https://doi. org/10.1097/MCC.0b013e3283356679

631. MentecH, Dupont H, Bocchetti Metal (2001) Upper digestive intolerance during enteral nutrition in critically ill patients: frequency, risk factors, and complications. Crit Care Med 29:1955-1961

632. Zaloga GP, Marik P (2000) Promotility agents in the intensive care unit. Crit Care Med 28:2657-2659

633. Tiancha H, Jiyong J, Min Y (2015) How to promote bedside placement of the postpyloric feeding tube: a network meta-analysis of randomized controlled trials. JPEN J Parenter Enteral Nutr 39:521-530

634. Alhazzani W, Almasoud A, Jaeschke R et al (2013) Small bowel feeding and risk of pneumonia in adult critically ill patients: a systematic review and metaanalysis of randomized trials. Crit Care 17:R127

635. Valenta J, Brodska H, Drabek T et al (2011) Highdose selenium substitution in sepsis: a prospective randomized clinical trial. Intensive Care Med 37:808-815

636. Bloos F, Trips E, Nierhaus A, Briegel J, Heyland DK, Jaschinski U, Moerer O, Weyland A, Marx G, Gründling M, Kluge S, Kaufmann I, Ott K, Quintel M, Jelschen F, Meybohm P, Rademacher S, MeierHellmann A, Utzolino $S$, Kaisers UX, Putensen C, Elke $\mathrm{G}$, Ragaller $\mathrm{M}$, Gerlach $\mathrm{H}$, Ludewig $\mathrm{K}$, Kiehntopf M, Bogatsch H, Engel C, Brunkhorst FM, Loeffler M, Reinhart K, SepNet Critical Care Trials Group Effect of Sodium Selenite Administration, Procalcitonin-Guided Therapy on Mortality in Patients With Severe Sepsis or Septic Shock (2016) A randomized clinical trial. JAMA Intern Med 176(9):1266-1276. https://doi.org/10.1001/ jamainternmed.2016.2514

637. SepNet Critical Care Trials Group, Bloos F, Trips E, Nierhaus A et al (2016) Effect of sodium selenite administration and procalcitonin-guided therapy on mortality in patients with severe sepsis or septic shock: a randomized clinical trial. JAMA Intern Med 176:1266-1276

638. Marik PE, Zaloga GP (2001) Early enteral nutrition in acutely ill patients: a systematic review. Crit Care Med 29:2264-2270

639. Avenell A (2006) Glutamine in critical care: current evidence from systematic reviews. Proc Nutr Soc 65:236-241

640. Avenell A (2009) Hot topics in parenteral nutrition. Current evidence and ongoing trials on the use of glutamine in critically-ill patients and patients undergoing surgery. Proc Nutr Soc 68:261-268

641. Jiang H, Chen W, Hu W et al (2009) The impact of glutamine-enhanced enteral nutrition on clinical Outcome of patients with critical illness: a systematic review of randomized controlled trials. Zhonghua Shao Shang Za Zhi 25:325-330

642. Novak F, Heyland DK, Avenell A et al (2002) Glutamine supplementation in serious illness: a systematic review of the evidence. Crit Care Med 30:2022-2029

643. Metabolism, Nutrition Working Group, SEMICYUC, Spain, Grau T, Bonet A, Miñambres E et al (2011) The effect of L-alanyl-L-glutamine dipeptide supplemented total parenteral nutrition on infectious morbidity and insulin sensitivity in critically ill patients. Crit Care Med 39:1263-1268

644. Wang Z, Forceville $X$, Van Antwerpen $P$ et al (2009) A large-bolus injection, but not continuous infusion of sodium selenite improves Outcome in peritonitis. Shock 32:140-146

645. Scandinavian Critical Care Trials Group, Wernerman J, Kirketeig T, Andersson B et al (2011) Scandinavian glutamine trial: a pragmatic multicentre randomised clinical trial of intensive care unit patients. Acta Anaesthesiol Scand 55:812-818

646. Canadian Critical Care Trials Group, Heyland D, Muscedere J, Wischmeyer PE et al (2013) A randomized trial of glutamine and antioxidants in critically ill patients. N Engl J Med 368:1489-1497

647. Beale RJ, Sherry T, Lei K et al (2008) Early enteral supplementation with key pharmaconutrients improves Sequential Organ Failure Assessment score in critically ill patients with sepsis: Outcome of a randomized, controlled, double-blind trial. Crit Care Med 36:131-144

648. Fuentes-Orozco C, Anaya-Prado R, GonzálezOjeda A et al (2004) L-alanylL-glutamine-supplemented parenteral nutrition improves infectious morbidity in secondary peritonitis. Clin Nutr 23:13-21

649. McRae MP (2017) Therapeutic benefits of glutamine: an umbrella review of meta-analyses. Biomed Rep 6:576-584

650. Stehle P, Ellger B, Kojic D, Feuersenger A, Schneid C, Stover J, Scheiner D, Westphal M (2017) Glutamine dipeptide-supplemented parenteral nutrition improves the clinical ouitcomes of critically ill patients: a systematic evaluation of randomized controlled trials. Clin Nutr ESPEN 17:75-85

651. Garcia de Acilu M, Leal S, Caralt B, Roca O, Sabater J, Masclans JR (2015) The role of omega-3 polyunsaturated fatty acids in the treatment of patients with acute respiratory distress syndrome: a clinical review. Biomed Res Int 2015:653750

652. NIH NHLBI Acute Respiratory Distress Syndrome Network of Investigators, NHLBI ARDSClinical Trials Network, Rice TW, Wheeler AP, Thompson BT et al (2011) Enteral omega-3 fatty acid, gammalinolenic acid, and antioxidant supplementation in acute lung injury. JAMA 306:1574-1581

653. Lu C, Sharma S, McIntyre L, Rhodes A, Evans L, Almenawer S, Leduc L, Angus DC, Alhazzani W (2017) Omega-3 supplementation in patients with sepsis: a systematic review and meta-analysis of randomized trials. Ann Intensive Care 7(1):58. https://doi.org/10.1186/s13613-017-0282-5

654. Chen H, Wang S, Zhao Y et al (2018) Correlation analysis of omega-3 fatty acids and mortality of sepsis and sepsis-induced ARDS in adults: data from previous randomized controlled trials. Nutr $J$ 17(1):57

655. Li WS, Chen ZZ, Zheng YJ, Zhong M, Ma JF, Xuan LZ, Song JQ, Lv QZ, Zhu DM (2018) The efficacy of parenteral fish oil in critical illness patients with sepsis: a prospective, nonrandomized, observational study. Asia Pac J Clin Nutr 27(2):306-312. https://doi.org/10.6133/ apjcn.032017.07 
656. Kreymann KG, Heyland DK, de Heer G, Elke G (2017) Intravenous fish oil in critically ill and surgical patients-Historical remarks and critical appraisal. Clin Nutr. https://doi.org/10.1016/j.clnu.2017.07. 006

657. White DB, Engelberg RA, Wenrich MD et al (2010) The language of prognostication in intensive care units. Med Decis Making 30:76-83

658. Chiarchiaro J, Buddadhumaruk P, Arnold RM et al (2015) Quality of communication in the ICU and surrogate's understanding of prognosis. Crit Care Med 43:542-548

659. Canadian Critical Care Trials Group, Downar J, You JJ, Bagshaw SM et al (2015) Nonbeneficial treatment Canada: definitions, causes, and potential solutions from the perspective of healthcare practitioners. Crit Care Med 43:270-281

660. Improving Palliative Care in the ICU (IPAL-ICU) Project Advisory Board, Nelson JE, Curtis JR, Mulkerin C et al (2013) Choosing and using screening criteria for palliative care consultation in the ICU: a report from the Improving Palliative Care in the ICU (IPAL-ICU) Advisory Board. Crit Care Med 41:2318-2327

661. Detering KM, Hancock AD, Reade MC et al (2010) The impact of advance care planning on end of life care in elderly patients: randomised con trolled trial. BMJ 340:c1345

662. Scheunemann LP, McDevitt M, Carson SS et al (2011) Randomized, con trolled trials of interventions to improve communication in intensive care: a systematic review. Chest 139:543-554

663. American Thoracic Society ad hoc Committee on Futile and Potentially Inappropriate Treatment, American Thoracic Society, American Association for Critical Care Nurses, American College of Chest Physicians, European Society for Intensive Care Medicine; Society of Critical Care, Bosslet GT, Bosslet GT, Pope TM, Rubenfeld GD et al (2015) An Official ATS/AACN/ACCP/ESICM/SCCM Policy Statement: Responding to Requests for Potentially Inappropriate Treatments in Intensive Care Units. Am JRespir Crit Care Med 191:1318-1330

664. American College of Critical Care Medicine, American Thoracic Society: Shared Decision Making in ICUs, Kon AA, Davidson JE, Morrison W et al (2016) An American college of critical care medicine and American thoracic society policy statement. Crit Care Med 44:188-201

665. Aslakson R, Cheng J, Vollenweider D et al (2014) Evidence-based pallia tive care in the intensive care unit: a systematic review of interven tions. J Palliat Med 17:219-235

666. Schulz V, Novick RJ (2013) The distinct role of palliative care in the sur gical intensive care unit. Semin Cardiothorac Vasc Anesth 17:240-248

667. Khandelwal N, Kross EK, Engelberg RA et al (2015) Estimating the effect of palliative care interventions and advance care planning on ICU utilization: a systematic review. Crit Care Med 43:1102-1111

668. DeCato TW, Engelberg RA, Downey L et al (2013) Hospital variation and temporal trends in palliative and end-of-life care in the ICU. Crit Care Med 41:1405-1411

669. Sprung CL, Truog RD, Curtis JR et al (2014) Seeking worldwide profes sional consensus on the principles of end-of-life care for the critically ill. The Consensus for Worldwide End-of-Life Practice for Patients in Intensive Care Units (WELPICUS) study. Am J Respir Crit Care Med 190:855-866

670. Flanders SA, Strasen JH (2014) Review of evidence about family pres ence during resuscitation. Crit Care Nurs Clin North Am 26:533-550
671. Oczkowski SJ, Mazzetti I, Cupido C et al (2015) The offering of family presence during resuscitation: a systematic review and meta-analysis. J Intensive Care 3:41

672. Oczkowski SJ, Mazzetti I, Cupido C et al (2015) Canadian critical care society: family presence during resuscitation: a Canadian critical care society position paper. Can Respir J 22:201:205VV

673. Heyland DK, Hopman W, Coo H, Tranmer J, McColl MA (2000) Long-term health-related quality of life in survivors of sepsis. Short Form 36: a valid and reliable measure of health-related quality of life. Crit Care Med 28(11):3599-3605

674. Korosec Jagodic H, Jagodic K, Podbregar M (2006) Long-term outcome and quality of life of patients treated in surgical intensive care: a comparison between sepsis and trauma. Crit Care 10(5):R134

675. Granja C, Dias C, Costa-Pereira A, Sarmento A (2004) Quality of life of survivors from severe sepsis and septic shock may be similar to that of others who survive critical illness. Crit Care 8(2):R91-R98

676. Bolton CF, Gilbert JJ, Hahn AF, Sibbald WJ (1984) Polyneuropathy in critically ill patients. J Neurol Neurosurg Psychiatry 47(11):1223-1231

677. Tepper M, Rakic S, Haas JA, Woittiez AJ (2000) Incidence and onset of critical illness polyneuropathy in patients with septic shock. Neth J Med 56(6):211-214

678. Davydow DS, Gifford JM, Desai SV, Bienvenu OJ, Needham DM (2009) Depression in general intensive care unit survivors: a systematic review. Intensive Care Med 35(5):796-809

679. Schelling G (2008) Post-traumatic stress disorder in somatic disease: lessons from critically ill patients. Prog Brain Res 167:229-237

680. Mehlhorn J, Freytag A, Schmidt K et al (2014) Crit Care Med 42(5):1263-1271

681. Graf J, Doig GS, Cook DJ, Vincent JL, Sibbald WJ (2002) Randomized, controlled clinical trials in sepsis: has methodologicalquality improved over time? Crit Care Med 30(2):461-472

682. Prescott HC, Angus DC (2018) Enhancing recovery from sepsis: a review. JAMA 319(1):62-75

683. S3-Leitlinie der Deutschen Gesellschaft für Anästhesiologie und Intensivmedizin e.V., der Deutschen Gesellschaft für Infektiologie e.V., der Deutschen Gesellschaft für Hygiene und Mikrobiologie e. V., der Deutschen Gesellschaft für Pneumologie und Beatmungsmedizin e.V., der Paul-Ehrlich Gesellschaft für Chemotherapie e.V, der Deutschen Röntgengesellschaft und der Gesellschaft für Virologie. Pneumologie 2018. 72(01): 15-63

684. S3-Leitlinie - Epidemiologie, Diagnostik und Therapie erwachsener Patienten mit nosokomialer Pneumonie - Update 2017 https://www. awmf.org/uploads/tx_szleitlinien/020-013I_S3_ Nosokomiale_Pneumonie_Erwachsener_201711.pdf (letzter Zugriff: 16. Jan. 2019)

685. Arabi YM et al (2019) N Engl J Med. https://doi.org/ 10.1056/NEJMoa1816150

686. Zhoe SX (2002) Acta Pharmacol Sin 23(7):654-658

687. Parker MM (1984) Ann Intern Med. https://doi.org/ 10.7326/0003-4819-100-4-483

688. Macchia A et al (2012) Crit Care Med 40:2768-2772

689. Rommers MK et al (2006) Crit Care 10:R93. https:// doi.org/10.1186/cc4952

690. Ukleja A (2010) Nutr Clin Pract 25:16-25

691. Luiking YC, Poeze M, Deutz NE (2015) Arginine infusion in patients with septic shock increases nitric oxide production without hemodynamic instability. Clin Sci 128:57-67

692. Tao et al (2016) BMC Anesthesiol. https://doi.org/ 10.1186/s12871-016-0200-7 